\title{
A FINITE ELEMENT FORMULATION OF ACTIVE CONSTRAINED-LAYER FUNCTIONALLY GRADED BEAM
}

\author{
by \\ Ry Long \\ B.Eng Mechanical Engineeing \\ Ryerson University 2008
}

A thesis

presented to Ryerson University

in partial fulfillment of the

requirements for the degree of

Master of Applied Science

in the Program of

Mechanical Engineering

Toronto, Ontario, Canada, 2010

(C) Ry Long, 2010 


\section{Author's Declaration}

I hereby declare that I am the sole author of this thesis.

I authorize Ryerson University to lend this thesis to other institutions or individuals for the purpose of scholarly research.

Ry Long

I further authorize Ryerson University to reproduce this thesis by photocopying or by other means, in total or in part, at the request of other institutions or individuals for the purpose of scholarly research.

Ry Long 


\title{
Abstract
}

\author{
A FINITE ELEMENT FORMULATION OF ACTIVE \\ CONSTRAINED-LAYER FUNCTIONALLY GRADED BEAM \\ (C) Ry Long, 2010 \\ Master of Applied Science \\ in the Program of \\ Mechanical Engineering \\ Ryerson University
}

Active constrained-layer damping (ACLD) treatment is the combination of passive and active features in the control of structural vibrations. A three-layer structure that consists of a functionally graded (FG) host beam, with a bonded viscoelastic layer and a constraining piezoelectric fiber-reinforce composite (PFRC) laminate is modeled and analyzed. The assumptions for modeling the system are the application of Timoshenko beam theory for the host beam and PFRC laminate, and a higher-order beam theory for the viscoelastic layer. The formulation is assumed to have field variables that are expressed as polynomials through the thickness of the structure and linear interpolation across the span. The extended Hamilton's principle is utilized to determine the system equations of motion, which are then solved using the Newmark time-integration scheme. Many support conditions such as fullyand partially-clamped cantilevered, partially clamped-clamped and simply-supported are analyzed. The effects of ply angle orientation, as well as FG properties, are also carefully examined. 


\section{Acknowledgments}

There are a number of people who sincerely deserve my thanks for their effort in the support of my master's thesis. Special thanks goes to Dr. Donatus Oguamanam for his guidance, patience, support and encouragement throughout the research and writing of this dissertation. He has exceeded all of my expectations of what an advisor should be. I thank him for his excellent technical direction, and more importantly, for his encouragement in my academic pursuits.

Many thanks to the work of my committee members for the reading of this thesis, making many important suggestions, and participating as members of my defense committee.

Last, but not the least, I would like to thank my parents for their constant support and great patience during my educational career, and my love, Oumar, for his encouragement in my academic endeavours, and every enjoyable moment we shared. 


\section{Table of Conents}

Author's Declaration $\quad$ ii

$\begin{array}{ll}\text { Abstract } & \text { iii }\end{array}$

Acknowledgments

Table of Contents $\quad$ v

List of Figures vi

List of Tables $\quad$ vii

Nomenclature viii

\begin{tabular}{llr}
\hline & Introductions & 1
\end{tabular}

1.1 Background $\ldots \ldots \ldots \ldots \ldots$

$1.1 .1 \quad$ Classical Three-Layer Theory . . . . . . . . . . . . . . . . 3

1.2 Research Objectives $\ldots \ldots \ldots \ldots \ldots$

1.3 Thesis Outline. . . . . . . . . . . . . . . . . . . . . . . . . 4

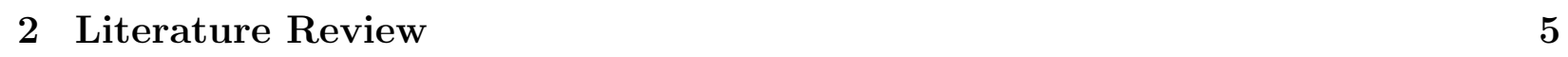

2.1 Active Constrained-Layer Damping ACLD . . . . . . . . . . . . 5

2.2 Piezoelectric Element as Actuator and Sensor $\ldots \ldots \ldots$. . . . . . . 6

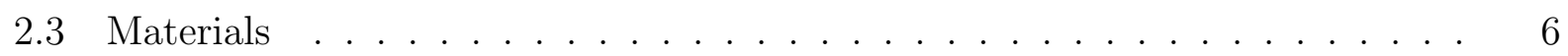

$2.3 .1 \quad$ Functionally Graded Material (FGM) Beam . . . . . . . . . . 7

$2.3 .2 \quad$ Viscoelastic Material . . . . . . . . . . . . . . . . . . . 8

$2.3 .3 \quad$ Piezoelectric Fiber-Reinforced Composite (PFRC) $\ldots \ldots \ldots$

\begin{tabular}{lll}
\hline 3 & Theoretical Formulation & 11
\end{tabular}

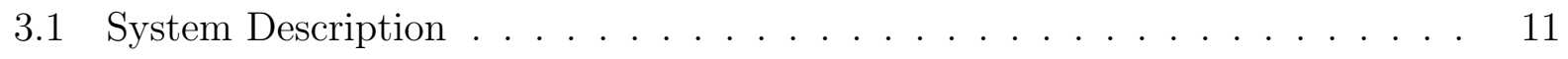


$3.2 \quad$ FGM Beam (Bottom Layer) $\ldots \ldots \ldots \ldots$

3.2 .1 Kinematic Assumptions $\ldots \ldots \ldots \ldots$

3.2 .2 Constitutive Equations $\ldots \ldots \ldots$

3.2 .3 Variational Formulation using Hamilton's principle $\ldots \ldots \ldots$. . . . 15

$3.2 .3 .1 \quad$ Kinetic Energy $\ldots \ldots \ldots \ldots \ldots \ldots$

3.2 .3 .2 Strain Energy $\ldots \ldots \ldots \ldots$

$3.3 \quad$ Viscoelastic layer (Core Layer) $\ldots \ldots \ldots$

3.3 .1 Kinematic Assumptions $\ldots \ldots \ldots \ldots$

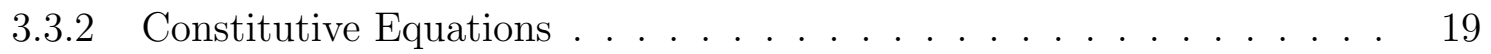

3.3.2.1 Elastic Properties . . . . . . . . . . . . . . . . . . 19

3.3 .2 .2 Viscoelastic Properties . . . . . . . . . . . . . . 20

3.3 .3 Variational Formulation using Hamilton's principle . . . . . . . . . . 21

$3.3 .3 .1 \quad$ Kinetic Energy $\ldots \ldots \ldots \ldots \ldots . \ldots \ldots$

3.3 .3 .2 Strain Energy . . . . . . . . . . . . . . . . . . 22

$3.4 \quad$ PFRC Laminate (Top Layer) $\ldots \ldots$. . . . . . . . . . . . . . . . . . 23

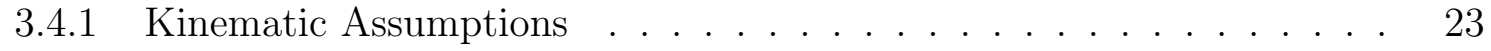

$3.4 .1 .1 \quad$ Mechanical Field Assumptions $\ldots \ldots \ldots$

3.4.1.2 Electrical Field Assumptions _... . . . . . . . . . . 24

3.4 .2 Constitutive Equations $\ldots \ldots \ldots \ldots$

$3.4 .2 .1 \quad$ Mechanical Properties . . . . . . . . . . . . . . . 25

$3.4 .2 .2 \quad$ Electrical Properties $\ldots \ldots \ldots \ldots$

3.4 .2 .3 Laminate Stiffness Matrix ABD . . . . . . . . . . . . . . . 30

3.4 .3 Variational Formulation using Hamilton's principle $\ldots \ldots \ldots$. . . . . 32

$3.4 .3 .1 \quad$ Kinetic Energy . . . . . . . . . . . . . . . . . . . . 32

$3.4 .3 .2 \quad$ Strain Energy $\ldots \ldots \ldots \ldots \ldots$

\begin{tabular}{|lll}
\hline & Finite Element Modeling & 35
\end{tabular}

4.1 Shape Functions $\ldots \ldots \ldots \ldots$. . . . . . . . . . . . . . . . . . . . . 37

4.1 .1 FGM Beam (Bottom Layer) $\ldots \ldots \ldots \ldots$

$4.1 .2 \quad$ Viscoelastic Layer (Core) $\ldots \ldots \ldots$

4.2 PRFC Laminate (Top Layer) $\ldots \ldots$. . . . . . . . . . . . . . . . . 40

4.3 External Force. . . . . . . . . . . . . . . . . . . . . . . . . . . . . . . . . 41

4.4 Equation of Motion $\ldots \ldots \ldots \ldots$

4.5 Time Integration $\ldots \ldots \ldots \ldots \ldots$ 
5 Numerical Simulation $\quad 43$

$5.1 \quad$ Formulation Comparison with Literature . . . . . . . . . . . . . . . . 43

5.1 .1 Sandwich Beam with FG core . . . . . . . . . . . . . . . 43

5.1 .2 Visco-Aluminum Sandwich Beam . . . . . . . . . . . . . . . 47

5.1 .2 .1 Visco-FGM Sandwich Beam . . . . . . . . . . . . . . . . 49

5.1.3 Cantilevered Beam with Viscoelastic Core and Piezoelectric Constraining Layer . . . . . . . . . . . . . . . . . . . . 53

5.2 Parametric Study . . . . . . . . . . . . . . . . . . . . . 57

5.2 .1 Effect of Ply Orientation . . . . . . . . . . . . . . . . 58

$5.2 .1 .1 \quad$ Fully-clamped Cantilevered Beam Case . . . . . . . . . . 58

$5.2 .1 .2 \quad$ Partially-clamped Cantilevered Beam Case. . . . . . . . . . 63

5.2.1.3 Comparison of Fully- and Partially- Clamped Cantilevered with Classical Method . . . . . . . . . . . . . . . . . 67

$5.2 .1 .4 \quad$ Partially Clamped-clamped Beam Case . . . . . . . . . . 71

5.2 .1 .5 Simply-supported Beam Case . . . . . . . . . . . 75

5.2 .2 Effect of FGM Properties $\ldots \ldots \ldots \ldots \ldots$

5.2.2.1 Fully- and Partially- Clamped Cantilevered Beam Cases . . 83

$5.2 .2 .2 \quad$ Partially Clamped-clamped Beam Case . . . . . . . . . . 93

$5.2 .2 .3 \quad$ Simply-supported Beam Case . . . . . . . . . . . . 103

$\begin{array}{lll}6 & \text { Summary, Conclusions and Recommendations } & 113\end{array}$

\begin{tabular}{lr}
\hline A Appendix & 116
\end{tabular}

A.1 MATLAB Codes . . . . . . . . . . . . . . . . . . . . . . 116 


\section{List of Figures}

1.1 Layer damping treatments: (a) free or unconstrained layer damping; (b) pas-

\begin{tabular}{|c|c|c|}
\hline \multicolumn{3}{|c|}{ sive constrained layer damping (PCLD); (c) active constrained layer damping } \\
\hline & $(\mathrm{ACLD})$. & 2 \\
\hline 2.1 & ACLD Actuator Sensor Configuration. & 6 \\
\hline 2.2 & Schematic diagram of a lamina made of PFRC [20]. & 10 \\
\hline
\end{tabular}

3.1 Schematic of ACLD beam. . . . . . . . . . . . . . . . . . . . . . . 12

3.2 Geometry of an $n$-layered laminate. [23] . . . . . . . . . . . . . . . . . . 30

4.1 Finite element model of ACLD beam. . . . . . . . . . . . . . . . . . . 36

$5.1 \quad$ Schematic of a fully-clamped sandwich beam with FG core. . . . . . . . . . . 44

5.2 Visco-aluminum sandwich beam. . . . . . . . . . . . . . . . . 47

$5.3 \quad$ Dynamic response of visco-aluminum sandwich beam. . . . . . . . . . . . . . 48

$5.4 \quad$ Visco-E-FGM sandwich beam with (zirconia/aluminum) $)_{S}$ orientation. . . . . 49

5.5 Visco-P-FGM (p=0.78) sandwich beam with (aluminum/zirconia) $)_{S}$ orientation. 49

$5.6 \quad$ Visco-E-FGM sandwich beam with (zirconia/aluminum) $)_{S}$ orientation. . . . . 51

$5.7 \quad$ Visco-P-FGM $(\mathrm{p}=1.28)$ sandwich beam with (zirconia/aluminum $)_{S}$ orientation. 51

$5.8 \quad$ Visco-E-FGM sandwich beam with (aluminum/zirconia) $)_{S}$ orientation. . . . . 52

$5.9 \quad$ Visco-P-FGM sandwich beam with (aluminum/zirconia) $)_{s}$ orientation. . . . . 52

5.10 Visco sandwich beam comparison with homogeneous and FG characteristics. 53

5.11 Cantilevered beam with viscoelastic core and piezoelectric constraining layer. 53

5.12 Tip deflections and control voltage with a PZT5H constraining layer for $h_{c}=$ $0.2 \mathrm{~mm}$. (a) Passive damping tip deflection. (b) Active-passive damping tip deflection. (c) Active-passive damping control voltage. . . . . . . . . . . . . 55

5.13 Tip deflections and control voltage with a PZT5H constraining layer for $h_{c}=$ $1.0 \mathrm{~mm}$. (a) Passive damping tip deflection. (b) Active-passive damping tip deflection. (c) Active-passive damping control voltage. . . . . . . . . . . . . 56

5.14 Beam with viscoelastic core and PFRC laminate constraining layer. . . . . . 57 
5.15 Fully-clamped cantilevered beam with ACLD treatment. . . . . . . . . . . . 58

5.16 Effect of ply orientation on the tip transverse displacement for uncontrolledpassive damping of fully-clamped cantilevered beam. (a) $0_{4}^{\circ}$, (b) $\left[0^{\circ} / 90^{\circ}\right]_{S}$, (c) $\left[45^{\circ} /-45^{\circ}\right]_{S},(\mathrm{~d})\left[30^{\circ} / 60^{\circ}\right]_{S} . \ldots \ldots \ldots$. . . . . . . . . . . . 60

5.17 Effect of ply orientation on the tip transverse displacement for controlledactive-passive damping of fully-clamped cantilevered beam. (a) $0_{4}^{\circ}$, (b) $\left[0^{\circ} / 90^{\circ}\right]_{S}$, (c) $\left[45^{\circ} /-45^{\circ}\right]_{S},(\mathrm{~d})\left[30^{\circ} / 60^{\circ}\right]_{S} . \ldots \ldots \ldots \ldots$. . . . . . . . . . . . 61

5.18 Effect of ply orientation on the actuator voltage for controlled-active-passive \begin{tabular}{|c|}
\hline damping of fully-clamped cantilevered beam. (a) $0_{4}^{\circ}$, (b) $\left[0^{\circ} / 90^{\circ}\right]_{S}$, (c) $\left[45^{\circ} /-\right.$ \\
\hline
\end{tabular} $\left.45^{\circ}\right]_{S},(\mathrm{~d})\left[30^{\circ} / 60^{\circ}\right]_{S} . \ldots \ldots \ldots \ldots$. . . . . . . . . . . . . . . 62

5.19 Partially-clamped cantilevered beam with ACLD treatment. . . . . . . . . . 63

5.20 Effect of ply orientation on the tip transverse displacement for uncontrolled\begin{tabular}{|c|}
\hline passive damping of partially-clamped cantilevered beam. (a) $0_{4}^{\circ}$, (b) $\left[0^{\circ} / 90^{\circ}\right]_{S}$, \\
\hline
\end{tabular} (c) $\left[45^{\circ} /-45^{\circ}\right]_{S},(\mathrm{~d})\left[30^{\circ} / 60^{\circ}\right]_{S}$. . . . . . . . . . . . . . . . . 64

5.21 Effect of ply orientation on the tip transverse displacement for controlled-

\begin{tabular}{|c|}
\hline active-passive damping of partially-clamped cantilevered beam. (a) $0_{4}^{\circ}$, (b) \\
\hline
\end{tabular}

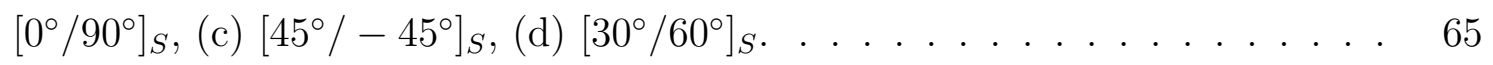

5.22 Effect of ply orientation on the actuation voltage for controlled-active-passive damping of partially-clamped cantilevered beam. (a) $0_{4}^{\circ}$, (b) $\left[0^{\circ} / 90^{\circ}\right]_{S}$, (c) $\left[\left[45^{\circ} /-45^{\circ}\right]_{S},(\mathrm{~d})\left[30^{\circ} / 60^{\circ}\right]_{S}\right.$. . . . . . . . . . . . . . . . . . 66

5.23 Comparison of tip transverse displacements for uncontrolled-passive fully- and partially- clamped cantilevered beams. (a) $0_{4}^{\circ}$, (b) $\left[0^{\circ} / 90^{\circ}\right]_{S}$, (c) $\left[45^{\circ} /-45^{\circ}\right]_{S}$,

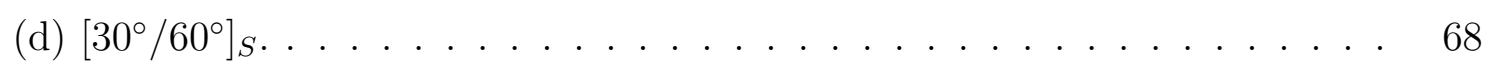

5.24 Comparison of tip transverse displacements for controlled-active-passive fullyand partially- clamped cantilevered beams. (a) $0_{4}^{\circ}$, (b) $\left[0^{\circ} / 90^{\circ}\right]_{S}$, (c) $\left[45^{\circ} /-\right.$ $\left.45^{\circ}\right]_{S},(\mathrm{~d})\left[30^{\circ} / 60^{\circ}\right]_{S} \ldots \ldots \ldots \ldots \ldots$. . . . . . . . . . . . . . 69

5.25 Comparison of actuation voltages for controlled-active-passive fully- and partiallyclamped cantilevered beams. (a) $0_{4}^{\circ}$, (b) $\left[0^{\circ} / 90^{\circ}\right]_{S}$, (c) $\left[45^{\circ} /-45^{\circ}\right]_{S}$, (d) $\left[30^{\circ} / 60^{\circ}\right]_{S}$. . . . . . . . . . . . . . . . . . . . . 70

5.26 Partially clamped-clamped beam with ACLD treatment. . . . . . . . . . . . 71

5.27 Effect of ply orientation on the mid-point transverse displacement for uncontrolled\begin{tabular}{|r|}
\hline passive damping of partially (bottom) clamped-clamped beam. (a) $0_{4}^{\circ}$, (b) \\
\hline$\left[0^{\circ} / 90^{\circ}\right]_{S},(\mathrm{c})\left[45^{\circ} /-45^{\circ}\right]_{S},(\mathrm{~d})\left[30^{\circ} / 60^{\circ}\right]_{S} . \ldots \ldots$. . . . . . . . . . . . 72
\end{tabular}

5.28 Effect of ply orientation on the mid-point transverse displacement for controlledactive-passive damping of partially (bottom) clamped-clamped beam. (a) $0_{4}^{\circ}$, (b) $\left[0^{\circ} / 90^{\circ}\right]_{S}$, (c) $\left[45^{\circ} /-45^{\circ}\right]_{S},(\mathrm{~d})\left[30^{\circ} / 60^{\circ}\right]_{S} \ldots \ldots \ldots . \ldots . \ldots 73$ 
5.29 Effect of ply orientation on the actuation voltage for controlled-active-passive damping of partially (bottom) clamped-clamped beam. (a) $0_{4}^{\circ}$, (b) $\left[0^{\circ} / 90^{\circ}\right]_{S}$, (c) $\left[45^{\circ} /-45^{\circ}\right]_{S},(\mathrm{~d})\left[30^{\circ} / 60^{\circ}\right]_{S}$. . . . . . . . . . . . . . . . 74

5.30 Simply-supported beam with ACLD treatment. . . . . . . . . . . . . . . 75

5.31 Effect of ply orientation on the mid-point transverse displacement for uncontrolledpassive damping of simply-supported beam. (a) $0_{4}^{\circ}$, (b) $\left[0^{\circ} / 90^{\circ}\right]_{S}$, (c) $\left[45^{\circ} /-\right.$

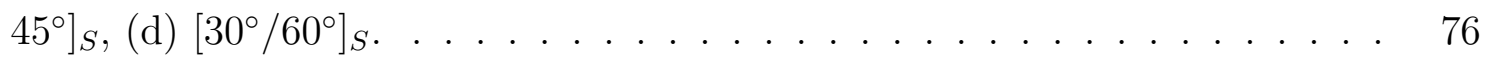

5.32 Effect of ply orientation on the mid-point transverse displacement for controlledactive-passive damping of simply-supported beam. (a) $0_{4}^{\circ}$, (b) $\left[0^{\circ} / 90^{\circ}\right]_{S}$, (c) $\left[45^{\circ} /-45^{\circ}\right]_{S},(\mathrm{~d})\left[30^{\circ} / 60^{\circ}\right]_{S}$. . . . . . . . . . . . . . . . 77

5.33 Effect of ply orientation on the actuation voltage for controlled-active-passive damping of simply-supported beam. (a) $0_{4}^{\circ}$, (b) $\left[0^{\circ} / 90^{\circ}\right]_{S}$, (c) $\left[45^{\circ} /-45^{\circ}\right]_{S}$,

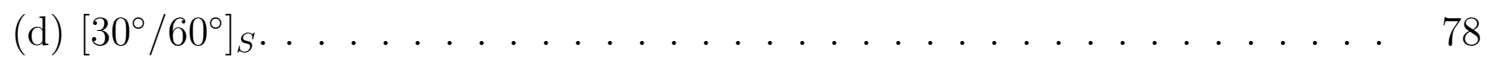

5.34 Variation of Young's modulus in a P-FGM and E-FGM beam for $E_{M}>E_{C}$. $\quad 80$

5.35 Variation of Young's modulus in a P-FGM and E-FGM beam for $E_{M}>E_{C}$. $\quad 81$

5.36 Variation of Young's modulus in a (a) P-FGM $\left(E_{M}<E_{C}\right)$ and E-FGM $\left(E_{M}>\right.$ $\left.E_{C}\right)$ and (b) E-FGM $\left(E_{M}<E_{C}\right)$ and P-FGM $\left(E_{M}>E_{C}\right)$ beam. . . . . . . . 82

5.37 Effect of change in average elastic modulus on the tip transverse displacement for uncontrolled-passive damping of (1a),(1b),(1c) fully-clamped and (2a),(2b),(2c) partially-clamped cantilevered beam. . . . . . . . . . . . . . 84

5.38 Effect of change in average elastic modulus on the tip transverse displacement for controlled-active-passive damping of (1a),(1b),(1c) fully-clamped and (2a), (2b),(2c) partially-clamped cantilevered beam. . . . . . . . . . . . . 85

5.39 Effect of change in average elastic modulus on the actuation voltage for controlled-active-passive damping of (1a),(1b),(1c) fully-clamped and (2a),(2b),(2c) partially-clamped cantilevered beam. . . . . . . . . . . . . . . 86

5.40 Effect of varying constituents moduli for fixed average elastic modulus on the tip transverse displacement for uncontrolled-passive damping of (1a),(1b),(1c) fully-clamped and (2a),(2b),(2c) partially-clamped cantilevered beam. . . . . 88

5.41 Effect of varying constituents moduli for fixed average elastic modulus on the tip transverse displacement for controlled-active-passive damping of (1a),(1b),(1c) fully-clamped and (2a),(2b),(2c) partially-clamped cantilevered beam. . . . . 89

5.42 Effect of varying constituents moduli for fixed average elastic modulus on the actuation voltage for controlled-active-passive damping of (1a),(1b),(1c) fully-clamped and (2a),(2b),(2c) partially-clamped cantilevered beam. . . . . 90 
5.43 Effect of exchange in metal and ceramic elastic moduli on the tip transverse displacement for uncontrolled-passive damping of (1a),(1b),(1c) fully-clamped and (2a),(2b),(2c) partially-clamped cantilevered beam. . . . . . . . . . . . . 92

5.44 Effect of change in average elastic modulus on the mid-point transverse displacement for uncontrolled-passive damping of partially clamped-clamped beam. (a),(b),(c) $E_{a v g}=200 \mathrm{GPa}, 230 \mathrm{GPa}$, and $260 \mathrm{GPa} . . . . . . .94$

5.45 Effect of change in average elastic modulus on the mid-point transverse displacement for controlled-active-passive damping of partially clamped-clamped

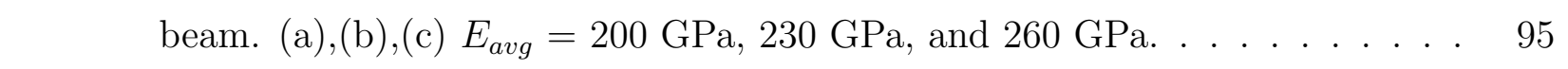

5.46 Effect of change in average elastic modulus on the actuation voltage for controlled-active-passive damping of partially clamped-clamped beam. (a),(b),(c) $E_{\text {avg }}=200 \mathrm{GPa}, 230 \mathrm{GPa}$, and 260 GPa. . . . . . . . . . . . . . . . . 96

5.47 Effect of varying constituents moduli for fixed average elastic modulus on the mid-point transverse displacement for uncontrolled-passive damping of

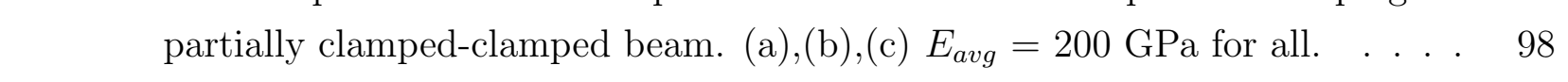

5.48 Effect of varying constituents moduli for fixed average elastic modulus on \begin{tabular}{|c|}
\hline the mid-point transverse displacement for controlled-active-passive damping \\
\hline of partially clamped-clamped beam. (a),(b),(c) $E_{\text {avg }}=200 \mathrm{GPa}$ for all. . . . 99
\end{tabular}

5.49 Effect of varying constituents moduli for fixed average elastic modulus on the actuation voltage for controlled-active-passive damping of partially clamped-

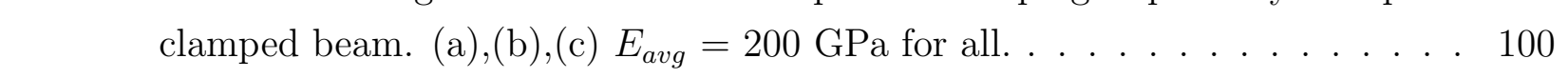

5.50 Effect of exchange in metal and ceramic elastic moduli on the mid-point trans\begin{tabular}{|c|}
\hline verse displacement for uncontrolled-passive damping of partially clamped- \\
\hline
\end{tabular}

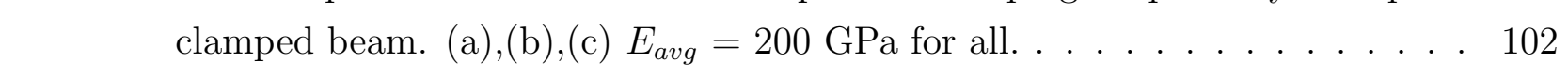

5.51 Effect of change in average elastic modulus on the mid-point transverse dis\begin{tabular}{|l}
\hline placement for uncontrolled-passive damping of simply-supported beam. (a),(b),(c) \\
\hline$E_{\text {avg }}=200 \mathrm{GPa}, 230 \mathrm{GPa}$, and $260 \mathrm{GPa}$. . . . . . . . . . . . . . . . 104
\end{tabular}

5.52 Effect of change in average elastic modulus on the mid-point transverse displacement for controlled-active-passive damping of simply-supported beam. (a),(b),(c) $E_{\text {avg }}=200 \mathrm{GPa}, 230 \mathrm{GPa}$, and $260 \mathrm{GPa}$. . . . . . . . . . . . . . 105

5.53 Effect of change in average elastic modulus on the actuation voltage for controlled-active-passive damping of simply-supported beam. (a),(b),(c) $E_{\text {avg }}$ $=200 \mathrm{GPa}, 230 \mathrm{GPa}$, and $260 \mathrm{GPa}$. . . . . . . . . . . . . . . . 106

5.54 Effect of varying constituents moduli for fixed average elastic modulus on the mid-point transverse displacement for uncontrolled-passive damping of simply-supported beam. (a),(b),(c) $E_{a v g}=200 \mathrm{GPa}$ for all. . . . . . . . . . . 108 
5.55 Effect of varying constituents moduli for fixed average elastic modulus on the mid-point transverse displacement for controlled-active-passive damping of simply-supported beam. (a),(b),(c) $E_{a v g}=200 \mathrm{GPa}$ for all. . . . . . . . . 109

5.56 Effect of varying constituents moduli for fixed average elastic modulus on the actuation voltage for controlled-active-passive damping of simply-supported beam. (a),(b),(c) $E_{\text {avg }}=200 \mathrm{GPa}$ for all. . . . . . . . . . . . . . . . . . . . . 110

5.57 Effect of exchange in metal and ceramic elastic moduli on the mid-point transverse displacement for uncontrolled-passive damping of simply-supported beam. (a),(b),(c) $E_{a v g}=200 \mathrm{GPa}$ for all. . . . . . . . . . . . . . . . . . . . . 112 


\section{List of Tables}

5.1 Material properties of sandwich beam with FG core. . . . . . . . . . . . . . . 44

5.2 First 10 natural frequencies of sandwich beam with a P-FGM $(\mathrm{p}=1)$ core. . 45

5.3 Fully-clamped cantilevered natural frequencies of sandwich beam with a PFGM $(\mathrm{p}=0.78)$ and E-FGM core for $E_{\text {avg }} \approx 105 G P a, \nu_{\text {avg }} \approx 0.3, \rho_{\text {avg }} \approx 4015$

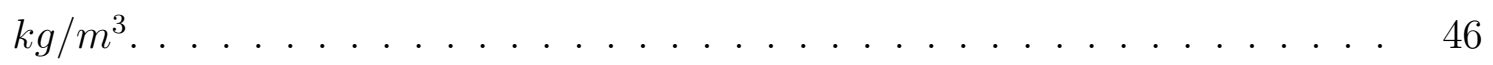

5.4 Partially-clamped cantilevered natural frequencies of sandwich beam with a PFGM $(\mathrm{p}=0.78)$ and E-FGM core for $E_{\text {avg }} \approx 105 G P a, \nu_{\text {avg }} \approx 0.3, \rho_{\text {avg }} \approx 4015$

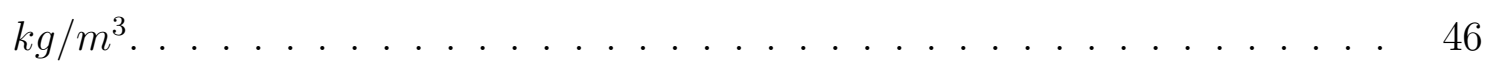

5.5 Material properties of visco-aluminum sandwich beam. . . . . . . . . . . 47

5.6 Mechanical properties and piezoelectric characteristics of the cantilever beam. 54

5.7 Mechanical properties and piezoelectric characteristics of the cantilever beam. 57

5.8 Variation of Young's modulus with respect to change in average elastic modulus. 79

5.9 Variation of Young's modulus with respect to fixed average elastic modulus

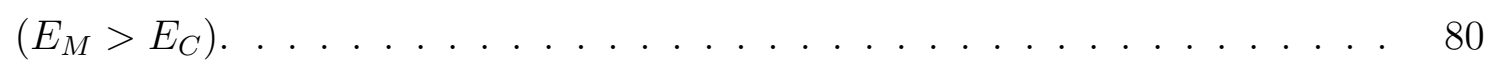

5.10 Exchange of ceramic and metal Young's modulus with respect to fixed average elastic modulus $\left(E_{M}<E_{C}\right) . \ldots \ldots \ldots \ldots \ldots$. . . . . . . 81 


\section{Nomenclature}

\begin{tabular}{|c|c|c|}
\hline VARIABLE & DEFINITION & UNITS \\
\hline $\mathrm{L}$ & Length of beam & $\mathrm{m}$ \\
\hline $\mathrm{b}$ & Width of beam & $\mathrm{m}$ \\
\hline$h_{i}$ & Thickness coordinate & $\mathrm{m}$ \\
\hline()$_{f}$ & Face sheet subscript & - \\
\hline()$_{b}$ & Bottom FGM beam subscript & - \\
\hline()$_{c}$ & Core (viscoelastic) subscript & - \\
\hline()$_{t}$ & Top PFRC laminate subscript & - \\
\hline$\rho$ & Density & $\mathrm{kg} / m^{3}$ \\
\hline$\nu$ & Poisson's Ratio & - \\
\hline $\mathrm{E}$ & Young's modulus of elasticity & $\mathrm{Pa}$ \\
\hline$x, z$ & Beam coordinate & - \\
\hline$\alpha$ & Fractional derivative order & - \\
\hline$\tau$ & Relaxation time & $\mathrm{s}$ \\
\hline$\Delta t$ & Time step & $\mathrm{s}$ \\
\hline $\bar{d}_{b, c, t}$ & Displacement vector & $\mathrm{m}$ \\
\hline $\bar{u}_{b, c, t}(x, z, t)$ & Axial displacement & $\mathrm{m}$ \\
\hline $\bar{w}_{b, c, t}(x, z, t)$ & Transverse displacement & $\mathrm{m}$ \\
\hline $\mathbf{u}_{b, c, t}$ & Local displacement vector & $\mathrm{m}$ \\
\hline$\widetilde{\mathbf{u}}_{c}$ & Anelastic displacement vector & $\mathrm{m}$ \\
\hline $\bar{\epsilon}_{b, c, t}$ & Strain vector & - \\
\hline$\widetilde{\bar{\epsilon}}_{c}$ & Core anelastic strain vector & - \\
\hline$\epsilon_{i}, \gamma_{i j}$ & Stain vectors & - \\
\hline$D_{b, c, t}$ & Derivative operator matrix & - \\
\hline $\bar{\sigma}_{b, c, t}$ & Stress vectors & $\mathrm{Pa}$ \\
\hline
\end{tabular}




\begin{tabular}{|c|c|c|}
\hline$\sigma_{i}, \tau_{i j}$ & Normal stress, shear stress & $\mathrm{Pa}$ \\
\hline$c_{i j}$ & Effective elastic coefficients & $\mathrm{Pa}$ \\
\hline$e_{i j}$ & Piezoelectric constants & $\mathrm{C} / m^{2}$ \\
\hline$d_{i j}$ & Dielectric permittivity & $\mathrm{F} / \mathrm{m}$ \\
\hline$Q_{b, c, t}$ & Elastic matrix & $\mathrm{Pa}$ \\
\hline $\bar{Q}_{t}$ & Top layer transformation matrix & $\mathrm{Pa}$ \\
\hline$\widetilde{Q}_{t}$ & Top layer reduced matrix & $\mathrm{Pa}$ \\
\hline$\xi_{b, c, t}$ & Coefficient matrix & - \\
\hline$\lambda$ & Functional material gradient & $m^{-1}$ \\
\hline$\omega$ & Natural frequency & $s^{-1}$ \\
\hline$E_{0}$ & Static modulus of elasticity & $\mathrm{Pa}$ \\
\hline$E_{\infty}$ & Dynamic modulus of elasticity & $\mathrm{Pa}$ \\
\hline$I_{b, c, t}$ & Moment of Inertia & $m^{2}$ \\
\hline$C_{b, c, t}$ & Elastic stiffness matrix & $\mathrm{Pa}$ \\
\hline$\widetilde{C}_{b, c, t}$ & Anelastic stiffness matrix & $\mathrm{Pa}$ \\
\hline V & Voltage & $\mathrm{V}$ \\
\hline$\psi$ & Electric potential & $\mathrm{V}$ \\
\hline$E_{z}$ & Electrical field & $\mathrm{V} / \mathrm{m}$ \\
\hline$D_{z}$ & Electrical displacement field & $\mathrm{C} / m^{2}$ \\
\hline $\mathrm{k}$ & Ply number & - \\
\hline$\theta$ & Ply orientation angle & $\operatorname{deg}$ \\
\hline$T_{k}$ & Transformation matrix & - \\
\hline$R$ & Reuter matrix & - \\
\hline$q_{e}$ & Element displacement vector & $\mathrm{m}$ \\
\hline$M_{b, c, t}$ & Mass matrix & $\mathrm{kg}$ \\
\hline$K_{b, t}$ & Stiffness matrix & $\mathrm{N} / \mathrm{m}$ \\
\hline$K_{c}$ & Core elastic stiffness matrix & $\mathrm{N} / \mathrm{m}$ \\
\hline$\widetilde{K}_{c}$ & Core anelastic stiffness matrix & $\mathrm{N} / \mathrm{m}$ \\
\hline$A_{i j}$ & Extensional stiffness component & $\mathrm{N} / \mathrm{m}$ \\
\hline$B_{i j}$ & Coupling stiffness component & $\mathrm{Nm} / \mathrm{m}$ \\
\hline$D_{i j}$ & Bending stiffness component & $\mathrm{Nm}$ \\
\hline$N_{x}^{t}$ & Electrical force resultant component & $\mathrm{N} / \mathrm{m}$ \\
\hline$M_{x}^{t}$ & Electrical moment resultant component & $\mathrm{Nm} / \mathrm{m}$ \\
\hline$\xi$ & Local coordinate & - \\
\hline
\end{tabular}




$\begin{array}{lll}N_{i \xi} & \text { Shape functions } & - \\ N_{b, c, t} & \text { Shape function matrix } & - \\ \mathrm{J} & \text { Jacobian } & - \\ \delta & \text { Variation function } & - \\ T_{b, c, t} & \text { Kinetic Energy } & \mathrm{J} \\ U_{b, c, t} & \text { Strain Energy } & \mathrm{J} \\ \widetilde{U}_{c} & \text { Anelastic strain energy } & \mathrm{J} \\ \mathrm{W} & \text { Work } & \mathrm{J} \\ \widetilde{W}_{c} & \text { Anelastic force work } & \mathrm{J} \\ \mathrm{F} & \text { Force vector } & \mathrm{N}\end{array}$




\section{Chapter 1}

\section{Introductions}

\section{$1.1 \quad$ Background}

Recent advances in design and manufacturing of composite structures have significantly enhanced their use in various applications including aerospace, automotive and civil industries. Machines and structures subjected to dynamic loading experience vibration, which, if unattended, could cause serious problems, lead to energy loss, material fatigue, noise and other failures. In order to achieve the best design with desirable performance, it is necessary to control the vibration, especially for lightweight structures. The successful reduction of vibration and noise will cause the structures to operate under lower stress levels, offering better performance and longer life.

A considerable amount of research has been done over the years with regard to the modeling and control of lightweight composite structures. The methods to overcome structural instability and to eliminate noise due to vibration include the isolation of the system from the source of vibration, the redesign of the system, the attachment of masses, and the applications of damping treatments to the structure [1. This thesis is about the addition of damping, which is typically the most appropriate method of suppressing vibration of randomly excited structures that are governed by resonant modes [1]. Generally, for thin-walled structures such as beams, cylinders, and plates, layers of materials are added to dissipate more energy. There are distinctively two structural types of damping treatments, free-layer (unconstrained) and constrained layer damping (CLD) treatments. Free-layer damping treatments only refer to passive damping treatments, while CLD treatments consists of both passive and active damping treatments, also known as passive constrained layer damping (PCLD) and active constrained layer damping (ACLD), respectively [1].

Unconstrained damping methods consist of the bonding of a passive viscoelastic layer to the outer surface of the host structure (Figure 1.13). The viscoelastic layer is considered 
passive due to its inability to respond to the system. It is subjected to both direct and shear strain caused by its damping layer, resulting in energy dissipation. Damping treatments based on employing direct strain should attach the damping layer furthest from the neutral axis since this location experiences the longest direct strains.

If a second layer, a so-called constraining layer, is bonded to the damping layer (viscoelastic material), the multi-layered beam is known as a passive constrained layer damped (PCLD) treatment as depicted in Figure 1.1b. The attachment of the constraining layer induces increased considerable shear stresses to the damping layer. Typically, the constraining layer in PCLD is made of an elastic material. PCLD treatments have been widely used to solve vibration problems in automotive, computer hardware, military, and aerospace industry [1]. These vibration solutions have led to many applications such as inlet guide vanes of jet engines, helicopter cabins, exhaust stacks, satellite structures, equipment panels, antenna structures, truss systems, and space stations, etc. [7].

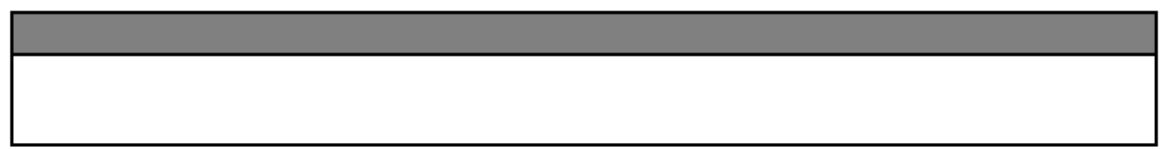

(a)

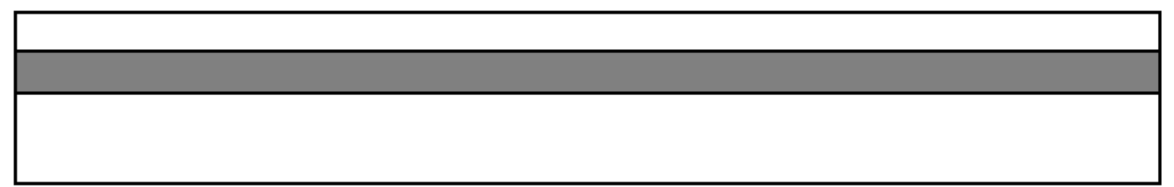

(b)

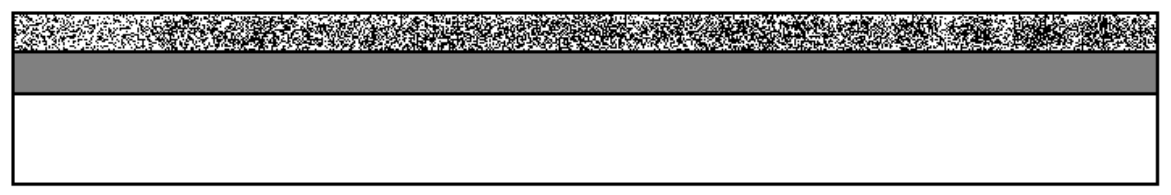

(c)

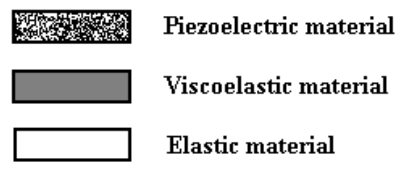

Figure 1.1: Layer damping treatments: (a) free or unconstrained layer damping; (b) passive constrained layer damping (PCLD); (c) active constrained layer damping (ACLD). 
Conversely, if an active element in the form of a piezoelectric material is attached to the passive viscoelastic material, it is known as an active constrained layer damped (ACLD) treatment. ACLD treatment is illustrated in Figure 1.1k. Here a sensor senses the vibrations of the structure and drives the piezoelectric actuator through a control system, enhancing the shear in the viscoelastic material. ACLD treatments are superior to PCLD treatments since they facilitate the control of low-frequency modes [1]. Additionally, ACLD treatments have the ability to adapt to changing environment in the structure. As a result of this adaptability, these structures are also considered as 'smart' or 'intelligent' structures.

\subsubsection{Classical Three-Layer Theory}

The classical theory is a commonly used theory for the analysis of a composite beams that focuses on the behavior of each individual layer. The assumptions which support the classical three-layer theory [2, 3, 4] are:

1. the host beam and the constraining layer deform as Euler-Bernoulli beams, that is, plane cross sections remain plane and perpendicular to the deflection curve of the deformed beam,

2. the viscoelastic core behaves as a Timoshenko beam,

3. the axial displacement field in each layer is linear through the thickness and the transverse displacement is constant throughout the thickness,

4. the core supports only shear load,

5. perfect bonding exists at the interfaces and no slip occurs between the layers,

6. in-plane inertia effects are ignored,

7. small-displacement theory is applicable.

To improve the model, shear and longitudinal energy contributions of the core will be considered as well as shear deformation in the top and bottom layers. To capture the shear contributions, the formulation in this thesis is based on a higher order beam theory for the core and Euler-Bernoulli and Timoshenko beam theory for the top and bottom layers.

\subsection{Research Objectives}

In the past, research has been done on the vibration analysis of an ACLD beam where the structure consisted of an elastic host beam as the base, a viscoelastic damping layer 
as the core and piezoelectric layer on top [6, 8]. A functionally graded (FG) host beam and a piezoelectric fiber reinforced composite (PFRC) top layer were adopted by Miroshnichenko [3]. The present work extends the model of an active constrained layer damping treatment with more representations of the FG host beam and PFRC top layer developed by Miroshnichenko. In Ref. [3], the author modeled the beam using the quasi-2D finite element formulation, whereas the present work uses a more simplified finite element formulation with the same quasi-2D concept. Additionally, it was assumed in Ref. [3] that the longitudinal displacement of both the host beam and core layer can be represented as cubic functions in the thickness coordinate, while their transverse displacements were quadratic functions. The longitudinal displacement of the top layer was modeled as a linear function in the thickness coordinate and the transverse displacement was constant. In this thesis, however, both the host beam and top layer are modeled using both Euler-Bernoulli and Timoshenko beam theories. The new modeling of the viscoelastic core follows the formulation of a higher-oder beam theory wherein the transverse and axial displacements are interpolated linearly and cubicly in the thickness coordinate, respectively.

The objective of this thesis is to analyze the ACLD beam using the proposed simplified quasi-2D formulation and compare it to the classical theory. The ACLD beam consists of a FG host beam, a viscoelastic core, and a PFRC top layer. Also, the effects of different representations of the FG beam with respect to Young's Modulus, Poisson's ratio, and density are investigated. The numerical simulations are performed in MATLAB.

\subsection{Thesis Outline}

This thesis is composed of 6 chapters.

Chapters 1 and 2 encompass background information, research objective, literature review, material concepts and properties of FGM, viscoelastic material, and PFRC laminates.

In Chapter 3, the mathematical formulation of the ACLD beam consisting of an FG beam using the simplified quasi-2D beam model is presented. Included in this are the system descriptions, kinematic assumptions, and constitutive equations for each layer.

Chapter 4 discusses the process of developing a finite element model. The extended Hamilton's principle is used to derive the dimensional equations of motion, which are solved using the Newmark time-integration method.

In chapter 5, simulation results are presented, and concluding remarks and suggestions for the future work are discussed in Chapter 6. 


\section{Chapter 2}

\section{Literature Review}

\subsection{Active Constrained-Layer Damping ACLD}

Active constrained-layer damping treatments (ACLD) have been examined to enhance the damping aptitude of the classical passive constrained-layer damping treatments (PCLD). Stanway et al. [1] discussed the progression from PCLD to ACLD, where a piezoelectric layer acts as the constraining layer. ACLD treatments greatly increase performance by enabling vibration control at low frequencies. With this advancement from PCLD to ACLD, vibration can now be controlled at both the high modes and low modes due to the passive and active elements, respectively. The elements of ACLD work in tandem in such a way that the active elements allow structures to adapt to changing environment and the passive elements provide a fail-safe mechanism. The active element consists of an actuator, usually in the form of a piezoelectric layer, which increases the beam's deformation. This, therefore, enhances the damping capability of the viscoelastic layer [1, 8, 9, 10].

Many researchers have been investigating the applications of ACLD treatments and their advantages in vibration control. Balamurugan and Narayanan [8] examined the development of a beam finite element model that has been partially covered with the ACLD treatment. Gao et al. [9] analyzed a simply-supported beam with enhanced self-sensing constrainedlayer (ECLD) treatments. The effect of edge element stiffness in ECLD on the sensing ability, loss factor and modal frequencies of the system were examined. The ECLD consisted of edge elements that were connected to both the host structure and piezoelectric layer for the purpose of transmissibility between the sensor and actuator. In [1], Stanway et al. highlighted practical applications of ACLD. They presented various actuator and sensor configurations that have been investigated by other researchers and also discussed the development of some modeling and control techniques. Trindade et al. [10] described numerous hybrid damping treatments, geometric configurations, modeling approaches and control algorithms of beams 
used in the literature. They also performed a thorough comparative analysis regarding the different hybrid active-passive damping configurations of other literature.

\subsection{Piezoelectric Element as Actuator and Sensor}

The piezoelectric element has both direct and inverse effects that enable its use as an actuator or a sensor. The direct effect is defined as the generation of an electric charge in proportion to an applied force, and the inverse effect induces an expansion or contraction under an applied electric field. Hence, a simplified ACLD configuration is developed with the benefit of the dual nature of the piezoelectric element as a self-sensing actuator [1, 9, 10].

A distinctive configuration that was examined by both Stanway et al. [1] and Trindade et al. [10], comprised of an elastic beam, a viscoelastic core, and a piezoelectric actuator as the constraining layer with a sensor that was bonded to the treatment. This thesis employs an ACLD arrangement that is similar to this configuration (presented in both Refs. [1] and [10]), where an accelerometer is used to measure the tip velocity of the beam and then fed into the controller of the system. The controller then sends a signal to excite the piezoelectric actuator. A schematic of the configuration is shown in Figure 2.1.
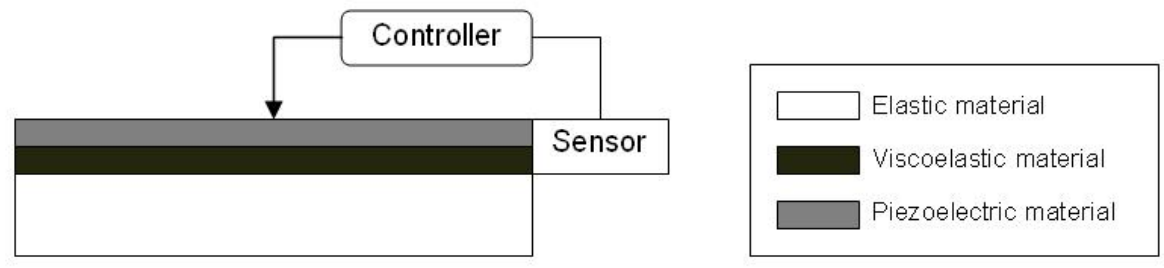

Figure 2.1: ACLD Actuator Sensor Configuration.

\subsection{Materials}

The present three-layer system consists of a host beam that is made of functionally graded material (FGM), with a bonded constraining viscoelastic layer and a piezoelectric fiberreinforced composite (PFRC) that covers the viscoelastic layer. Each material and its properties are described in the following subsections. 


\subsubsection{Functionally Graded Material (FGM) Beam}

Functionally graded materials (FGMs) consist of two or more material components whose relative volume fractions and microstructures are engineered to have gradually varying properties. FGMs are not like laminates which have sudden transition in material properties across the interface between distinct materials. This abrupt change in material properties can result in delamination due to large interlaminar stresses, and the initiation and propagation of cracks because of large plastic deformation at the interfaces [12]. FGMs are able overcome these adverse effects since they are engineered to have a smooth spatial variation of material properties.

During the past decade a large amount of research has been done to analyze the behavior of FGMs. For example, Amarani et al. [13] presented a free vibration analysis on a cantilevered sandwich beam with a functionally graded (FG) core. The Young's modulus, Poisson's ratio, and density varied in the thickness following the power-law or rule of mixture technique. The element-free Galerkin method and standard Galerkin formulation for two dimensional elasticity problems were considered. Rahmani et al. [14] also studied the free vibration of sandwiched beams with a flexible functionally graded syntactic core. A new model based on high-order sandwich panel theory was implemented. The formulations consisted of the classical beam theory for the face sheets and an elasticity theory for the FG core. It was concluded that increasing the inhomogeneity of the core led to higher natural frequencies of the beam modes.

Apetre et al. [15] performed an impact response of a sandwich beam with functionally graded core. The variation of the Young's modulus of the core was represented by a polynomial in the thickness coordinate, but the Poisson's ratio was kept constant. A combination of Fourier analysis and Galerkin method was used to solve the system governing equations. The core thicknesses of the FG core were chosen such that the flexural stiffness of the sandwich beam were equal to that of the beam with a homogeneous core. The results indicate that functionally graded cores can be used effectively to mitigate or avoid impact damage in sandwich structures.

Ray and Sachade [12] derived the exact solution for the static analysis of FG plates integrated with a PFRC layer. The Young's modulus of the FG plate was assumed to vary exponentially across the thickness of the plate, while the Poisson's ratio was constant over the domain of the plate. The through-the-thickness behavior of the plates revealed bendingextension coupling in the FG plates even if the PFRC layer is not subjected to an applied voltage.

In this thesis, the Poisson's ratio $\rho$, density $\nu$, and Young's modulus $E$ vary throughout

the thickness of the beam. Two different functions of FGMs are investigated: power-law 
functions (P-FGM), and exponential functions (E-FGM). In Ref. [17], the E-FGM function and P-FGM function are respectively interpreted as:

$$
G(z)=G_{2} e^{\lambda(z+h)}
$$

where the functional material gradient is given as $\lambda=\frac{1}{2 h} \ln \left(G_{1} / G_{2}\right)$, and

$$
G(z)=f(z) G_{1}+[1-f(z)] G_{2}
$$

The volume fraction obeying the power-law is:

$$
f(z)=\left(\frac{z+h}{2 h}\right)^{p}
$$

where $p$ is the material parameter and $2 h$ is the thickness of the plate or beam. $G_{1}$ and $G_{2}$ are the specified material property at the bottom $(z=-h)$ and top surfaces $(z=h)$ of the FGM plate or beam, respectively.

\subsubsection{Viscoelastic Material}

Viscoelastic materials are often incorporated into light/flexible structures to reduce structural vibrations and noise. There are numerous techniques associated with incorporating viscoelastic materials into structures. A viscoelastic solid has a weak frequency dependence on its dynamic properties over an extensive frequency range [18]. The Golla-Hughes-McTavish (GHM) model is a method used for time-domain analysis of viscoelastic analysis of viscoelastic structures [8]. In Ref. [19], the Prony series method, a classical time-based technique for fitting creep or relaxation data in the form of exponential functions, is compared to a fractional derivative model. The fractional derivative model is another time-domain method which is based on fractional calculus. It is not commonly used in commercial finite element software such as ANSYS, which employs the Prony series method. The reason for this is most likely due to the increased mathematical difficulty even though it is a better viscoelastic modeling technique.

This thesis uses a four-parameter fractional derivative model to analyze the ACLD beam. Over the last two decades, the concept of fractional derivative has become an extremely effective device to describe the weak frequency dependence of most viscoelastic materials [18]. Galucio et al. [18] showed that the complex modulus of a one-dimensional model can be written as:

$$
\sigma(t)+\tau^{\alpha} \frac{d^{\tau} \sigma(t)}{d t^{\alpha}}=E_{0} \epsilon(t)+\tau^{\alpha} E_{\infty} \frac{d^{\alpha} \epsilon(t)}{d t^{\alpha}}
$$


where $\sigma$ and $\epsilon$ are the stress and the strain, $E_{0}$ and $E_{\infty}$ are the relaxed and instantaneous elastic moduli, and $\tau$ is the relaxation time. A Fourier transformation of Eq. 2.4 yields the elastic complex modulus:

$$
E^{*}(\omega)=\frac{\sigma^{*}(\omega)}{\epsilon^{*}(\omega)}=\frac{E_{0}+E_{\infty}(i \omega \tau)^{\alpha}}{1+(i \omega \tau)^{\alpha}}
$$

where $\omega^{*}$ and $\epsilon^{*}$ are the Fourier transforms of $\sigma(t)$ and $\epsilon(t)$, respectively; $E_{0}=E^{*}(\omega \rightarrow 0)$ represents the static modulus of elasticity; $E_{\infty}=E^{*}(\omega \rightarrow \infty)$ is the dynamic modulus; $\tau$ is the relaxation time; and $\alpha$ is the fractional derivative order. To fulfill the second law of thermodynamics, the conditions $0<\alpha<1, \tau>0$ and $E_{\infty}>E_{0}$ must hold.

\subsubsection{Piezoelectric Fiber-Reinforced Composite (PFRC)}

Piezoelectric materials have been extensively used as sensors and/or actuators and when merged into a high-performance lightweight smart structure, they actively control vibration. Piezoelectric sensors and/or actuators are either mounted on or embedded in the structure, enabling them to have self-controlling and self-monitoring capabilities. Even though piezoelectric materials play a major role in achieving active damping in structures, they possess low control authority because their monolithic piezoelectric stress/strain constants are quite small in magnitude [20]. Since the active damping of these structures are dependent on the piezoelectric stress/strain constants, modifications to these properties are desirous in order to improve their damping characteristics. Piezoelectric composites are effective in improving flexural vibration control if their fibers are longitudinally oriented to execute a bending mode of actuators. The fibers are subjected to a constant electric field in the direction transverse to the fiber direction, wherein the electric field is assumed to be the same for both the fibers and the matrix [20, 12]. The schematic diagram of a lamina made of PFRC is shown in Figure 2.2. 


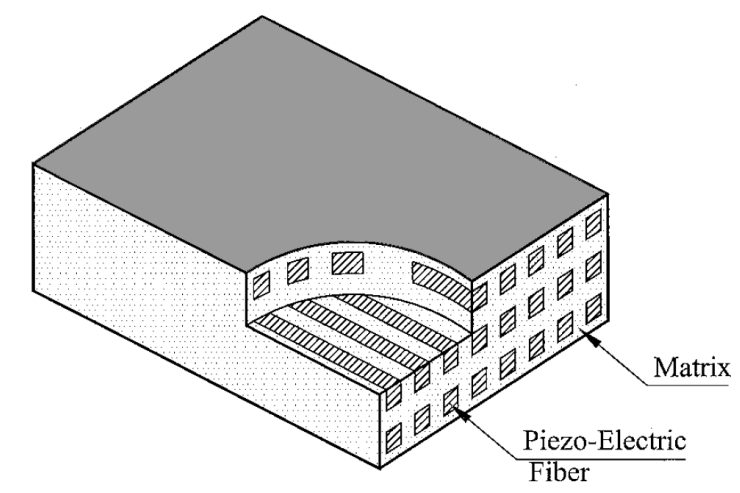

Figure 2.2: Schematic diagram of a lamina made of PFRC [20].

Investigations that include the implementation of PFRCs in the high-performance lightweight structures were performed by Mallik and Ray [20], and Ray and Sachade [12]. The former showed that the effective piezoelectric coefficient becomes significantly larger than the corresponding coefficient of the piezoelectric material of the fiber. The authors were able to determine the effectiveness based on a specific fiber volume fraction by using a micromechanical analysis. In Ref. [12], Ray and Sachade derived the exact solutions for the static analysis of FG plates integrated with a layer of PFRC material. They showed that the activated PFRC layer was more effective in controlling the deformations of the FG plates when the layer was attached to the surface of the FG plate the minimum stiffness rather than that with the maximum stiffness.

The inverse and direct constitutive equations of the PFRC material with respect to plane co-ordinates $(x y z)$ are given as [21]:

$$
\begin{aligned}
\{\sigma\}_{k} & =[\bar{Q}]_{k}\{\epsilon\}_{k}-[\bar{e}]_{k}^{T}\{E\}_{k} \\
\{D\}_{k} & =[\bar{e}]_{k}\{\epsilon\}_{k}-[\bar{d}]_{k}^{T}\{E\}_{k}
\end{aligned}
$$

where $k$ is the ply order in the laminate. $\{D\},\{E\},\{\epsilon\}$ and $\{\sigma\}$ are the electric displacement, electric field, strain and stress vectors, $[\bar{Q}],[\bar{e}]$ and $[\bar{d}]$ are the elastic, piezoelectric and permittivity constant matrices, respectively. 


\section{Chapter 3}

\section{Theoretical Formulation}

\subsection{System Description}

The system being analyzed is a rectangular three-layered composite beam. The host beam is made of FGM with through-the-thickness varying material properties (Young's modulus, Poisson's ratio, and density). The FGM beam is bonded to a viscoelastic material whose other side is bonded to a PFRC laminate layer. Figure 3.1 is a schematic of the ACLD beam arrangement where a sensor measures the tip velocity, which is then fed through the controller to obtain a voltage that is applied to the PFRC laminate. The bottom layer, represented by the host beam which is an FGM beam, is identified by the subscript $b$. The core layer, made of viscoelastic material, is distinguished by the subscript $c$. The top layer, representing the PFRC laminate, is identified by the subscript $t$ with $n$ number of plies. Each PFRC ply has unidirectional fibers aligned at an angle $\theta$ with respect to the reference axis. The geometric parameters of the ACLD beam are the length of the beam $L$, width of the beam $b$, thickness of the bottom layer (host beam) $2 h_{b}$, thickness of the core layer (viscoelastic layer) $2 h_{c}$, and thickness of the top layer (PFRC laminate) $2 h_{t}$. Each layer has its own axes where $z_{b}, z_{c}$, and $z_{t}$ are zero at the midsurface of each layer. The material properties of the $i^{t h}$ layer are density $\rho_{i}$, Young's Modulus $E_{i}$, and Poisson's ratio $\nu_{i}$. Perfect bonding is assumed at each interface where the adhesive material is thin with infinite stiffness. 


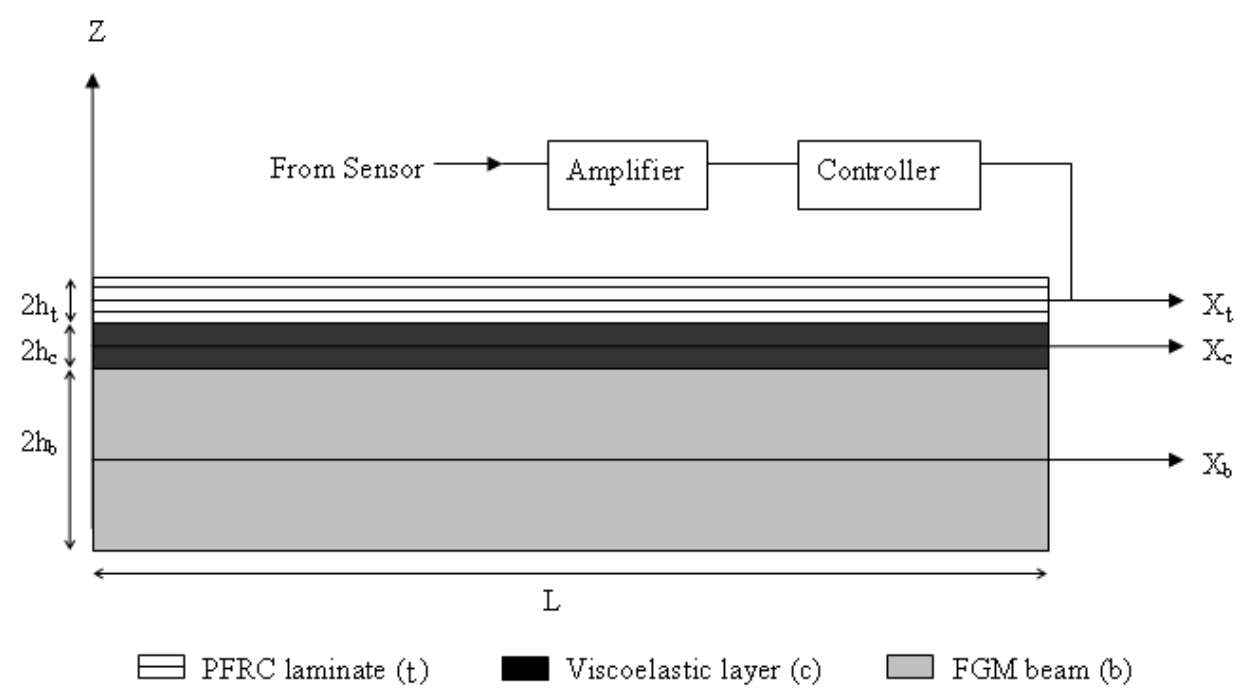

Figure 3.1: Schematic of ACLD beam.

\subsection{FGM Beam (Bottom Layer)}

\subsubsection{Kinematic Assumptions}

The FGM beam has an axial displacement that is linearly interpolated across the beam thickness and a through-the-thickness independent transverse displacement represented by $\bar{u}_{b}$ and $\bar{w}_{b}$, respectively. The displacement vector of the FGM beam $\bar{d}_{b}$ is written as:

$$
\bar{d}_{b}=\left\{\begin{array}{c}
\bar{u}_{b}\left(x, z_{b}, t\right) \\
\bar{w}_{b}\left(x, z_{b}, t\right)
\end{array}\right\}=\left\{\begin{array}{c}
u_{b}(x, t)-z_{b} \phi_{b}(x, t) \\
w_{b}(x, t)
\end{array}\right\}
$$

This kinematic assumption captures Euler-Bernoulli beam theory if, $\phi_{b}(x, t)=\frac{\partial w_{b}(x, t)}{\partial x}$. The reference point is taken at the geometric midpoint of the FGM beam. Defining a new vector $\mathbf{u}_{b}^{T}=\left\{u_{b} w_{b} \phi_{b}\right\}$ gives the following expansion of $\bar{d}_{b}$ :

$$
\bar{d}_{b}=\left[\begin{array}{ccc}
1 & 0 & -z_{b} \\
0 & 1 & 0
\end{array}\right]\left\{\begin{array}{l}
u_{b} \\
w_{b} \\
\phi_{b}
\end{array}\right\} \equiv\left[Z_{b}\right] \mathbf{u}_{b}
$$

Using linear strain-displacement relations gives the following: 


$$
\bar{\epsilon}_{b}=\left\{\begin{array}{c}
\epsilon_{x}\left(x, z_{b}, t\right) \\
\gamma_{x z}\left(x, z_{b}, t\right)
\end{array}\right\}_{b}=\left\{\begin{array}{c}
\frac{\partial \bar{u}_{b}}{\partial x} \\
\frac{\partial \bar{u}_{b}}{\partial z_{b}}+\frac{\partial \bar{w}_{b}}{\partial x}
\end{array}\right\}=\left\{\begin{array}{c}
\frac{\partial u_{b}}{\partial x}(x, t)-z_{b} \frac{\partial \phi_{b}}{\partial x}(x, t) \\
-\phi_{b}(x, t)+\frac{\partial w_{b}}{\partial x}(x, t)
\end{array}\right\}
$$

Note that for Euler-Bernoulli beam theory, $\gamma_{x z}=\frac{\partial \bar{u}_{b}}{\partial z_{b}}+\frac{\partial \bar{w}_{b}}{\partial x}=0$. The strain vector $\bar{\epsilon}_{b}$ can be written in matrix notation as:

$$
\bar{\epsilon}_{b}=\left\{\begin{array}{c}
\epsilon_{x} \\
\gamma_{x z}
\end{array}\right\}_{b}=\left[\begin{array}{cccc}
1 & -z_{b} & 0 & 0 \\
0 & 0 & -1 & 1
\end{array}\right]\left[D_{b}\right]\left\{\begin{array}{c}
u_{b} \\
w_{b} \\
\phi_{b}
\end{array}\right\} \equiv\left[\widetilde{Z}_{b}\right]\left[D_{b}\right] \mathbf{u}_{b}
$$

where the derivative operator matrix $\left[D_{b}\right]$ is given as:

$$
\left[D_{b}\right]=\left[\begin{array}{ccc}
\frac{\partial}{\partial x} & 0 & 0 \\
0 & 0 & \frac{\partial}{\partial x} \\
0 & 0 & 1 \\
0 & \frac{\partial}{\partial x} & 0
\end{array}\right]
$$

\subsubsection{Constitutive Equations}

With respect to an orthotropic FGM where the principal material properties coincide with both $x$ and $z$ axes, the 2-dimensional stress-strain constitutive relations are:

$$
\left\{\begin{array}{c}
\sigma_{x} \\
\sigma_{z} \\
\tau_{x z}
\end{array}\right\}_{b}=\left[\begin{array}{ccc}
c_{11}(z) & c_{13}(z) & 0 \\
c_{11}(z) & c_{33}(z) & 0 \\
0 & 0 & c_{55}(z)
\end{array}\right]\left\{\begin{array}{c}
\epsilon_{x} \\
\epsilon_{z} \\
\gamma_{x z}
\end{array}\right\}_{b}
$$

where $\sigma_{x}$ and $\sigma_{z}$ are the normal stresses in the $x$ and $z$ directions, respectively, $\gamma_{x z}$ is the shear stress in the $x z$-plane and $c_{i j}$ are elastic constants of the material. With the assumption that stress in the $z$ direction is zero $\left(i . e . \sigma_{z}=0\right)$, then $\epsilon_{z}=-\frac{c_{11}}{c_{33}} \epsilon_{x}$ and introducing $\bar{c}_{11}=$ $\left(c_{11}-\frac{c_{13}^{2}}{c_{33}}\right)$, the reduced constitutive relation is written as:

$$
\bar{\sigma}_{b}=\left\{\begin{array}{c}
\sigma_{x} \\
\tau_{x z}
\end{array}\right\}_{b}=\left[\begin{array}{cc}
\bar{c}_{11}(z) & 0 \\
0 & c_{55}(z)
\end{array}\right]\left\{\begin{array}{c}
\epsilon_{x} \\
\gamma_{x z}
\end{array}\right\}_{b} \equiv\left[Q_{b}(z)\right] \bar{\epsilon}_{b}
$$


For varying Young's modulus, the elasticity matrix may be written as:

$$
\left[Q_{b}(z)\right]=E_{b}(z)\left[\xi_{b}\right]=\frac{E_{b}(z)}{\left(1+\nu_{b}\right)\left(1-2 \nu_{b}\right)}\left[\begin{array}{cc}
1-\nu_{b} & 0 \\
0 & \left(1-2 \nu_{b}\right) / 2
\end{array}\right]
$$

where $E_{b}(z)$ is the elastic function that varies throughout the thickness and $\left[\xi_{b}\right]$ is the coefficient matrix. The isotropic elasticity matrix is related to the Young's modulus $E_{b}$ and Poisson's ratio $\nu_{b}$ and is under plane strain assumption. $\nu_{b}$ can either be constant or vary along z (i.e. $\left.\nu_{b}=\nu_{b}(z)\right)$.

Depending on the application, the FGM beam may have its Young's modulus, Poisson's ratio, and/or density varying continuously in the thickness direction, along the $z$-axis (i.e. $E=E(z), \nu=\nu(z), \rho=\rho(z))$. The formulation for two types of volume fraction methods: 1) power-law FGM (P-FGM) and 2) exponential FGM (E-FGM) may be written as:

1) In P-FGM the volume fraction is assumed to obey the power-law function:

$$
f(z)=\left(\frac{z+h}{2 h}\right)^{p}
$$

where $p$ is the material parameter and $2 h$ is the thickness of the layer. The rule of mixture is applied with the volume fraction $f(z)$ to determine the effective material property.

$$
G(z)=f(z) G_{b}^{0}+[1-f(z)] G_{b}^{1}
$$

2) In E-FGM the volume fraction obeys the exponential function:

$$
\begin{gathered}
G(z)=G_{b}^{0} e^{\lambda(z+h)} \\
\text { with } \lambda=\frac{1}{2 h} \ln \left(\frac{G_{b}^{1}}{G_{b}^{0}}\right)
\end{gathered}
$$

where $\mathrm{G}(\mathrm{z})$ represents any varying property (i.e. $G(z)=E=E(z), G(z)=\nu=\nu(z)$, or $G(z)=\rho=\rho(z))$. $G_{b}^{0}$ and $G_{b}^{1}$ represent the corresponding material properties at the bottom and top surfaces of the FGM beam, respectively, and $\lambda$ is a parameter that describes the inhomogeneity of the FGM beam throughout the thickness.

Thus, from Eqs. 3.9 and 3.10, the FGM elasticity, Poisson ratio and density equations for both P-FGM and E-FGM become:

1) P-FGM

$$
E_{b}(z)=\left(\frac{z+h}{2 h}\right)^{p} E_{b}^{0}+\left[1-\left(\frac{z+h}{2 h}\right)^{p}\right] E_{b}^{1}
$$




$$
\begin{aligned}
& \nu_{b}(z)=\left(\frac{z+h}{2 h}\right)^{p} \nu_{b}^{0}+\left[1-\left(\frac{z+h}{2 h}\right)^{p}\right] \nu_{b}^{1} \\
& \rho_{b}(z)=\left(\frac{z+h}{2 h}\right)^{p} \rho_{b}^{0}+\left[1-\left(\frac{z+h}{2 h}\right)^{p}\right] \rho_{b}^{1}
\end{aligned}
$$

2) E-FGM

$$
\begin{aligned}
& E_{b}(z)=E_{b}^{0} e^{\frac{1}{2 h} \ln \left(\frac{E_{b}^{1}}{E_{b}^{0}}\right)(z+h)} \\
& \nu_{b}(z)=\nu_{b}^{0} e^{\frac{1}{2 h} \ln \left(\frac{\nu_{b}^{1}}{\nu_{b}^{0}}\right)(z+h)} \\
& \rho_{b}(z)=\rho_{b}^{0} e^{\frac{1}{2 h} \ln \left(\frac{\rho_{b}^{1}}{\rho_{b}^{0}}\right)(z+h)}
\end{aligned}
$$

\subsubsection{Variational Formulation using Hamilton's principle}

The equation of motion is derived by utilizing the extended Hamilton's principle:

$$
\int_{t}(\delta T-\delta U+\delta W) d t=0
$$

where $\delta T\left(=\delta T_{b}+\delta T_{c}+\delta T_{t}\right)$ and $\delta U\left(=\delta U_{b}+\delta U_{c}+\delta U_{t}\right)$ are the variations of the kinetic energy and strain energy, respectively. $\delta W$ is the virtual work done by external forces on the system.

\subsubsection{Kinetic Energy}

The first variation of kinetic energy for the FGM beam is:

$$
\delta T_{b}=\int_{v} \rho_{b} \delta \bar{d}_{b}^{T} \ddot{\bar{d}}_{b} d v
$$

Substituting for $\bar{d}_{b}$ from Eq. 3.2 yields:

$$
\begin{array}{r}
\delta T_{b}=\rho_{b} \int_{x} \int_{y} \delta \mathbf{u}_{b}^{T}\left[I_{b}\right] \ddot{\mathbf{u}}_{b} d y d x \\
=\rho_{b} b \int_{x} \delta \mathbf{u}_{b}^{T}\left[I_{b}\right] \ddot{\mathbf{u}}_{b} d x
\end{array}
$$

where $\rho_{b}$ is either constant or varying (i.e. $\left.\rho_{b}=\rho_{b}(z)\right)$ with respect to P-FGM method (Eq. 
3.14) or E-FGM method (Eq. 3.17). For a constant density, the inertia matrix $\left[I_{b}\right]$ becomes:

$$
\left[I_{b}\right]=\int_{z}\left(\left[Z_{b}\right]^{T}\left[Z_{b}\right]\right) d z=\int_{z}\left[\begin{array}{ccc}
1 & 0 & -z_{b} \\
0 & 1 & 0 \\
-z_{b} & 0 & z_{b}^{2}
\end{array}\right] d z
$$

If the density is varying through the thickness, it must be integrated with the inertia matrix with respect to z $\left(\right.$ i.e. $\left.\int_{z} \rho_{b}\left[Z_{b}\right]^{T}\left[Z_{b}\right] d z\right)$.

\subsubsection{Strain Energy}

The variational strain energy for the FGM beam is:

$$
\delta U_{b}=\int_{v} \delta \bar{\epsilon}_{b}^{T} \bar{\sigma}_{b} d v
$$

Substituting for $\bar{\sigma}_{b}$ from Eq. 3.6 yields:

$$
\delta U_{b}=\int_{v} \delta \bar{\epsilon}_{b}^{T}\left[Q_{b}(z)\right] \bar{\epsilon}_{b} d v
$$

where $Q_{b}(z)$ varies according to P-FGM or E-FGM method, Eq. 3.12 and Eq. 3.15, respectively. Substituting for $\bar{\epsilon}_{b}$ from Eq. 3.4, yields:

$$
\delta U_{b}=b \int_{x} \delta \mathbf{u}_{b}^{T}\left[D_{b}\right]^{T}\left[C_{b}\right]\left[D_{b}\right] \mathbf{u}_{b} d x
$$

where the stiffness matrix $\left[C_{b}\right]$ is written as:

$$
\left[C_{b}\right]=\int_{z}\left(\left[\widetilde{Z}_{b}\right]^{T}\left[Q_{b}\right]\left[\widetilde{Z}_{b}\right]\right) d z
$$

\subsection{Viscoelastic layer (Core Layer)}

\subsubsection{Kinematic Assumptions}

Different from the FGM beam layer kinematics, the viscoelastic layer kinematics have axial and transverse displacements that are interpolated through the thickness by cubic and quadratic polynomial functions, respectively. The displacement vector $\bar{d}_{c}$ is characterized as: 


$$
\bar{d}_{c}=\left\{\begin{array}{c}
\bar{u}_{c}\left(x, z_{c}, t\right) \\
\bar{w}_{c}\left(x, z_{c}, t\right)
\end{array}\right\}=\left\{\begin{array}{c}
C_{1}\left(u_{b}(x, t)-h_{b} \phi_{b}(x, t)\right)+C_{2} u_{c 2}+C_{3} u_{c 3}+C_{4}\left(u_{t}(x, t)+h_{t} \phi_{t}(x, t)\right) \\
E_{1} w_{b}(x, t)+E_{2} w_{c}(x, t)+E_{3} w_{t}(x, t)
\end{array}\right\}
$$

where $u_{c 2}=\bar{u}_{c}\left(x, z_{c}=-\frac{h_{c}}{3}, t\right), \quad u_{c 3}=\bar{u}_{c}\left(x, z_{c}=\frac{h_{c}}{3}, t\right)$,

$$
\begin{aligned}
& C_{1}=-\frac{9}{16 h_{c}^{3}}\left(z_{c}^{3}-h_{c} z_{c}^{2}-\frac{1}{9} h_{c}^{2} z_{c}+\frac{1}{9} h_{c}^{3}\right), \quad C_{2}=\frac{27}{16 h_{c}^{3}}\left(z_{c}^{3}-\frac{1}{3} h_{c} z_{c}^{2}-h_{c}^{2} z_{c}+\frac{1}{3} h_{c}^{3}\right), \\
& C_{3}=-\frac{27}{16 h_{c}^{3}}\left(z_{c}^{3}+\frac{1}{3} h_{c} z_{c}^{2}-h_{c}^{2} z_{c}-\frac{1}{3} h_{c}^{3}\right), \quad C_{4}=\frac{9}{16 h_{c}^{3}}\left(z_{c}^{3}+h_{c} z_{c}^{2}-\frac{1}{9} h_{c}^{2} z_{c}-\frac{1}{9} h_{c}^{3}\right) \\
& E_{1}=\frac{z_{c}\left(z_{c}-h_{c}\right)}{2 h_{c}^{2}}, \quad E_{2}=\frac{h_{c}^{2}-z_{c}^{2}}{h_{c}^{2}}, \text { and } E_{3}=\frac{z_{c}\left(z_{c}+h_{c}\right)}{2 h_{c}^{2}}
\end{aligned}
$$

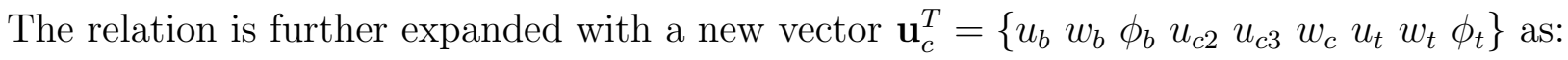

$$
\bar{d}_{c}=\left[\begin{array}{cccccccccc}
C_{1} & 0 & -h_{b} C_{1} & C_{2} & C_{3} & 0 & C_{4} & 0 & h_{t} C_{4} \\
0 & E_{1} & 0 & 0 & 0 & E_{2} & 0 & E_{3} & 0
\end{array}\right]\left\{\begin{array}{c}
u_{b} \\
w_{b} \\
\phi_{b} \\
u_{c 2} \\
u_{c 3} \\
w_{c} \\
u_{t} \\
w_{t} \\
\phi_{t}
\end{array}\right\} \equiv\left[Z_{c}\right] \mathbf{u}_{c}
$$

Using linear strain-displacement relations gives the following:

$$
\bar{\epsilon}_{c}=\left\{\begin{array}{c}
\epsilon_{x} \\
\epsilon_{z} \\
\gamma_{x z}
\end{array}\right\}_{c}=\left\{\begin{array}{c}
\frac{\partial \bar{u}_{c}}{\partial x} \\
\frac{\partial \bar{w}_{c}}{\partial z_{c}} \\
\frac{\partial \bar{u}_{c}}{\partial z_{c}}+\frac{\partial \bar{w}_{c}}{\partial x}
\end{array}\right\}
$$




$$
=\left\{\begin{array}{c}
C_{1} \frac{\partial u_{b}}{\partial x}-h_{b} C_{1} \frac{\partial \phi_{b}}{\partial x}+C_{2} \frac{\partial u_{c 2}}{\partial x}+C_{3} \frac{\partial u_{c 3}}{\partial x}+C_{4} \frac{\partial u_{t}}{\partial x}+h_{t} C_{4} \frac{\partial \phi_{b}}{\partial x} \\
\frac{\partial E_{1}}{\partial z_{c}} w_{b}+\frac{\partial E_{2}}{\partial z_{c}} w_{c}+\frac{\partial E_{3}}{\partial z_{c}} w_{t} \\
\frac{\partial C_{1}}{\partial z_{c}} u_{b}-h_{b} \frac{\partial C_{1}}{\partial z_{c}} \phi_{b}+\frac{\partial C_{2}}{\partial z_{c}} u_{c 2}+\frac{\partial C_{3}}{\partial z_{c}} u_{c 3}+\frac{\partial C_{4}}{\partial z_{c}} u_{t}+h_{t} \frac{\partial C_{4}}{\partial x} \phi_{b}+E_{1} \frac{\partial w_{b}}{\partial x}+E_{2} \frac{\partial w_{c}}{\partial x}+E_{3} \frac{\partial w_{t}}{\partial x}
\end{array}\right\}
$$

The strain vector $\bar{\epsilon}_{c}=\left\{\begin{array}{c}\epsilon_{x} \\ \epsilon_{z} \\ \gamma_{x z}\end{array}\right\}_{c}$ takes the following compact matrix notation:

$$
\bar{\epsilon}_{c}=\left[\widetilde{Z}_{c}\right]\left[D_{c}\right]\left\{\begin{array}{c}
u_{b} \\
w_{b} \\
\phi_{b} \\
u_{c 2} \\
u_{c 3} \\
w_{c} \\
u_{t} \\
w_{t} \\
\phi_{t}
\end{array}\right\} \equiv\left[\widetilde{Z}_{c}\right]\left[D_{c}\right] \mathbf{u}_{c}
$$

where $\left[\widetilde{Z}_{c}\right]$ and the derivative operator matrix $\left[D_{c}\right]$ are given as::

$$
\left[\widetilde{Z}_{c}\right]=\left[\begin{array}{ll}
{\left[\widetilde{Z}_{c 1}\right]} & {\left[\widetilde{Z}_{c 2}\right]}
\end{array}\right]
$$

where

$$
\left[\widetilde{Z}_{c 1}\right]=\left[\begin{array}{ccccccccc}
C_{1} & -h_{b} C_{1} & C_{2} & C_{3} & C_{4} & h_{t} C_{4} & 0 & 0 & 0 \\
0 & 0 & 0 & 0 & 0 & 0 & \frac{\partial E_{1}}{\partial z_{c}} & \frac{\partial E_{2}}{\partial z_{c}} & \frac{\partial E_{3}}{\partial z_{c}} \\
0 & 0 & 0 & 0 & 0 & 0 & 0 & 0 & 0
\end{array}\right]
$$

and

$$
\left[\widetilde{Z}_{c 2}\right]=\left[\begin{array}{ccccccccc}
0 & 0 & 0 & 0 & 0 & 0 & 0 & 0 & 0 \\
0 & 0 & 0 & 0 & 0 & 0 & 0 & 0 & 0 \\
\frac{\partial C_{1}}{\partial z_{c}} & -h_{b} \frac{\partial C_{1}}{\partial z_{c}} & \frac{\partial C_{2}}{\partial z_{c}} & \frac{\partial C_{3}}{\partial z_{c}} & \frac{\partial C_{4}}{\partial z_{c}} & h_{t} \frac{\partial C_{4}}{\partial z_{c}} & E_{1} & E_{2} & E_{3}
\end{array}\right]
$$




$$
\left[D_{c}\right]=\left[\begin{array}{ccccccccc}
\frac{\partial}{\partial x} & 0 & 0 & 0 & 0 & 0 & 0 & 0 & 0 \\
0 & 0 & \frac{\partial}{\partial x} & 0 & 0 & 0 & 0 & 0 & 0 \\
0 & 0 & 0 & \frac{\partial}{\partial x} & 0 & 0 & 0 & 0 & 0 \\
0 & 0 & 0 & 0 & \frac{\partial}{\partial x} & 0 & 0 & 0 & 0 \\
0 & 0 & 0 & 0 & 0 & 0 & \frac{\partial}{\partial x} & 0 & 0 \\
0 & 0 & 0 & 0 & 0 & 0 & 0 & 0 & \frac{\partial}{\partial x} \\
0 & 1 & 0 & 0 & 0 & 0 & 0 & 0 & 0 \\
0 & 0 & 0 & 0 & 0 & 1 & 0 & 0 & 0 \\
0 & 0 & 0 & 0 & 0 & 0 & 0 & 1 & 0 \\
1 & 0 & 0 & 0 & 0 & 0 & 0 & 0 & 0 \\
0 & 0 & 1 & 0 & 0 & 0 & 0 & 0 & 0 \\
0 & 0 & 0 & 1 & 0 & 0 & 0 & 0 & 0 \\
0 & 0 & 0 & 0 & 1 & 0 & 0 & 0 & 0 \\
0 & 0 & 0 & 0 & 0 & 0 & 1 & 0 & 0 \\
0 & 0 & 0 & 0 & 0 & 0 & 0 & 0 & 1 \\
0 & \frac{\partial}{\partial x} & 0 & 0 & 0 & 0 & 0 & 0 & 0 \\
0 & 0 & 0 & 0 & 0 & \frac{\partial}{\partial x} & 0 & 0 & 0 \\
0 & 0 & 0 & 0 & 0 & 0 & 0 & \frac{\partial}{\partial x} & 0
\end{array}\right]
$$

\subsubsection{Constitutive Equations}

\subsubsection{Elastic Properties}

The 2-dimensional stress-strain constitutive relation is:

$$
\left\{\begin{array}{c}
\sigma_{x} \\
\sigma_{z} \\
\tau_{x z}
\end{array}\right\}_{c}=\left[\begin{array}{ccc}
c_{11} & c_{13} & 0 \\
c_{11} & c_{33} & 0 \\
0 & 0 & c_{55}
\end{array}\right]\left\{\begin{array}{c}
\epsilon_{x} \\
\epsilon_{z} \\
\gamma_{x z}
\end{array}\right\}_{c} \equiv\left[Q_{c}\right] \bar{\epsilon}_{c}
$$

where $\sigma_{x}$ and $\sigma_{z}$ are the normal stresses in the $x$ and $z$ directions, respectively, $\gamma_{x z}$ is the shear stress in the $x z$-plane and $c_{i j}$ are elastic constants of the material. For an isotropic material and under plane strain assumption, the elasticity matrix can be written as:

$$
\left[Q_{c}\right]=E_{c}\left[\xi_{c}\right]
$$


where

$$
\left[\xi_{c}\right]=\frac{1}{\left(1+\nu_{c}\right)\left(1-2 \nu_{c}\right)}\left[\begin{array}{ccc}
1-\nu_{c} & \nu_{c} & 0 \\
\nu_{c} & 1-\nu_{c} & 0 \\
0 & 0 & \left(1-2 \nu_{c}\right) / 2
\end{array}\right]
$$

\subsubsection{Viscoelastic Properties}

The mechanical behavior of the viscoelastic material at any given time depends on the current state of stress and strain as well as on the full history of the material. Following Galucio et al. [18], the constitutive relation can be mathematically described by a two-dimensional constitutive equation as:

$$
\bar{\sigma}_{c}(t)+\tau^{\alpha} \frac{d^{\alpha} \bar{\sigma}_{c}(t)}{d t^{\alpha}}=E_{0}\left[\xi_{c}\right] \bar{\epsilon}_{c}(t)+\tau^{\alpha} E_{\infty}\left[\xi_{c}\right] \frac{d^{\alpha} \bar{\epsilon}_{c}(t)}{d t^{\alpha}}
$$

where $\left[\xi_{c}\right]$ is the same as Eq. 3.31, which assumes a plane strain condition, $E_{0}$ and $E_{\infty}$ are the relaxed and non-relaxed elastic moduli, $\tau$ is the relaxation time, and $\alpha$ is the fractional derivative order $(0<\alpha<1)$. The fractional operator $\frac{d^{\alpha}}{d t^{\alpha}}$ is approximated by the Grünwald definition by finite difference as:

$$
\frac{d^{\alpha} f(t)}{d t^{\alpha}} \approx \Delta t^{-\alpha} \sum_{j=0}^{N_{t}} A_{j+1} f(t-j \Delta t)
$$

where $\Delta t=\frac{t}{N}$ is the time step increment, $N_{t}$ is the total number of terms where $N_{t}<N$, and $A_{j+1}$ are the Grünwald coefficients given by the recurrence formula:

$$
A_{j+1}=\frac{j-\alpha-1}{j} A_{j}=\prod_{p=1}^{j} \frac{p-\alpha-1}{p}
$$

The anelastic strain $\widetilde{\bar{\epsilon}}_{c}$ at a specific time $t$ can be written as:

$$
\widetilde{\bar{\epsilon}}_{c}(t)=\bar{\epsilon}_{c}(t)-\left[\xi_{c}\right]^{-1} \frac{\bar{\sigma}_{c}}{E_{\infty}}
$$

This allows the constitutive equation, Eq. 3.32 to be rewritten as:

$$
\widetilde{\bar{\epsilon}}_{c}(t)+\tau^{\alpha} \frac{d^{\alpha} \widetilde{\bar{\epsilon}}_{c}(t)}{d t^{\alpha}}=\frac{E_{\infty}-E_{0}}{E_{\infty}} \bar{\epsilon}_{c}(t)
$$

This change in variable simplifies the equation by reducing the number of fraction derivative terms from two to one. Employing the Grünwald approximation from Eq. 3.33 and noting 
that $A_{1}=1$, Galucio at al. [18] showed that Eq. 3.35 gives the following form:

$$
\widetilde{\bar{\epsilon}}_{c}(t)=(1-\eta) \frac{E_{\infty}-E_{0}}{E_{\infty}} \bar{\epsilon}_{c}(t)-\eta \sum_{j=1}^{N_{t}}\left(\prod_{p=1}^{j} \frac{p-\alpha-1}{p}\right) \widetilde{\bar{\epsilon}}_{c}(t)(t-j \Delta t)
$$

where $\eta=\frac{\tau^{\alpha}}{\tau^{\alpha}+\Delta t^{\alpha}}$ is a dimensionless constant.

A contrived anelastic displacement vector $\left\{\widetilde{\mathbf{u}}_{c}(t)\right\}$ is expressed such that the anelastic strain can be written as:

$$
\widetilde{\bar{\epsilon}}_{c}(t)=\left[\widetilde{Z}_{c}\right]\left[D_{c}\right] \widetilde{\mathbf{u}}_{c}(t)
$$

Substituting $\bar{\epsilon}_{c}(t)$ from Eq. 3.28 and $\widetilde{\bar{\epsilon}}_{c}(t)$ from Eq. 3.38 at a specific time t into Eq. 3.37, the anelastic displacement becomes:

$$
\widetilde{\mathbf{u}}_{c}(t)=(1-\eta) \frac{E_{\infty}-E_{0}}{E_{\infty}} \mathbf{u}_{c}(t)-\eta \sum_{j=1}^{N_{t}}\left(\prod_{p=1}^{j} \frac{p-\alpha-1}{p}\right) \widetilde{\mathbf{u}}_{c}(t-j \Delta t)
$$

Isolating $\bar{\sigma}_{c}(t)$ from Eq. 3.35 yields:

$$
\bar{\sigma}_{c}(t)=E_{\infty}\left[\xi_{c}\right]\left(\bar{\epsilon}_{c}(t)-\widetilde{\bar{\epsilon}}_{c}(t)\right)
$$

Now, replacing $\widetilde{\bar{\epsilon}}_{c}(t)$ in Eq. 3.37 yields an updated expression of $\bar{\sigma}_{c}(t)$ at a given time t:

$$
\bar{\sigma}_{c}(t)=E_{0}\left[\xi_{c}\right]\left[\left(1+\eta \frac{E_{\infty}-E_{0}}{E_{0}}\right) \bar{\epsilon}_{c}(t)+\eta \frac{E_{\infty}}{E_{0}} \sum_{j=1}^{N_{t}}\left(\prod_{p=1}^{j} \frac{p-\alpha-1}{p}\right) \widetilde{\bar{\epsilon}}_{c}(t-j \Delta t)\right]
$$

Note that for an elastic material, $\eta=0$ and $E_{0}=E_{c}$ resulting in the reduction of Eq. 3.41 to: $\bar{\sigma}_{c}(t)=E_{0}\left[\xi_{c}\right] \bar{\epsilon}_{c}(t) \equiv\left[Q_{c}\right] \bar{\epsilon}_{c}(t)$. The constitutive relation for the viscoelastic core becomes:

$$
\bar{\sigma}_{c}(t)=\left[Q_{c}\right]\left[\left(1+\eta \frac{E_{\infty}-E_{0}}{E_{0}}\right) \bar{\epsilon}_{c}(t)+\eta \frac{E_{\infty}}{E_{0}} \sum_{j=1}^{N_{t}}\left(\prod_{p=1}^{j} \frac{p-\alpha-1}{p p}\right) \widetilde{\bar{\epsilon}}_{c}(t-j \Delta t)\right]
$$

\subsubsection{Variational Formulation using Hamilton's principle}

\subsubsection{Kinetic Energy}

The variation of kinetic energy for the viscoelastic layer is:

$$
\delta T_{c}=\int_{v} \rho_{c} \delta \bar{d}_{c}^{T} \ddot{\bar{d}}_{c} d v
$$


Substituting for $\bar{d}_{c}$ from Eq. 3.26 yields:

$$
\begin{aligned}
\delta T_{c} & =\rho_{c} \int_{x} \int_{y} \delta \mathbf{u}_{c}^{T}\left[I_{c}\right] \ddot{\mathbf{u}}_{c} d y d x \\
& =\rho_{c} b \int_{x} \delta \mathbf{u}_{c}^{T}\left[I_{c}\right] \ddot{\mathbf{u}}_{c} d x
\end{aligned}
$$

where the inertia matrix $\left[I_{c}\right]$ is expressed as:

$$
\left[I_{c}\right]=\int_{z}\left(\left[Z_{c}\right]^{T}\left[Z_{c}\right]\right) d z
$$

\subsubsection{Strain Energy}

The variational strain energy for the viscoelastic core at a given time $t$ is:

$$
\delta U_{c}=\int_{v} \delta \bar{\epsilon}_{c}^{T} \bar{\sigma}_{c}^{T} d v
$$

Substituting for $\bar{\sigma}_{c}$ from Eq. 3.42 yields:

$$
\begin{aligned}
\delta U_{c} & =\int_{v} \delta \bar{\epsilon}_{c}^{T}\left[Q_{c}\right] \bar{\epsilon}_{c} d v+\int_{v} \delta \bar{\epsilon}_{c}^{T}\left[\widetilde{Q}_{c}\right] \bar{\epsilon}_{c} d v \\
& +\eta \frac{E_{\infty}}{E_{0}} \sum_{j=1}^{N_{t}}\left(\prod_{p=1}^{j} \frac{p-\alpha-1}{p}\right) \times \int_{v} \delta \bar{\epsilon}_{c}^{T}\left[Q_{c}\right] \widetilde{\bar{\epsilon}}_{c}(t-j \Delta t) d v
\end{aligned}
$$

where $\left[\widetilde{Q}_{c}\right]=\eta \frac{E_{\infty}-E_{0}}{E_{0}}\left[Q_{c}\right]$.

The total viscoelastic strain energy can be rewritten as:

$$
\delta U_{c}=\delta \bar{U}_{c}+\delta \widetilde{U}_{c}+\delta \widetilde{W}_{c}
$$

where $\delta \bar{U}_{c}$ is the variation of the elastic strain energy, $\delta \widetilde{U}_{c}$ represents the variation of the anelastic strain energy, and $\delta \widetilde{W}_{c}$ is the virtual work done by the induced for in the viscoelastic layer. The variation of the elastic strain energy $\delta \bar{U}_{c}$ is determined by substituting the strain vector $\bar{\epsilon}_{c}$ from Eq. 3.28 into the strain energy equation:

$$
\delta \bar{U}_{c}=b \int_{x} \delta \mathbf{u}_{c}^{T}\left[D_{c}\right]^{T}\left[C_{c}\right]\left[D_{c}\right] \mathbf{u}_{c} d x
$$


where the elastic stiffness matrix $\left[C_{c}\right]$ is written as:

$$
\left[C_{c}\right]=\int_{z}\left(\left[\widetilde{Z}_{c}\right]^{T}\left[Q_{c}\right]\left[\widetilde{Z}_{c}\right]\right) d z
$$

Correspondingly, the variation of the anelastic strain energy $\delta \widetilde{\bar{U}}_{c}$ is written as:

$$
\delta \widetilde{\bar{U}}_{c}=b \int_{x} \delta \mathbf{u}_{c}^{T}\left[D_{c}\right]^{T}\left[\widetilde{C}_{c}\right]\left[D_{c}\right] \mathbf{u}_{c} d x
$$

where the anelastic stiffness matrix $\left[\widetilde{C}_{c}\right]$ is expressed as:

$$
\left[\widetilde{C}_{c}\right]=\eta \frac{E_{\infty}-E_{0}}{E_{0}}\left[C_{c}\right]
$$

Finally, the virtual work $\delta \widetilde{W}_{c}$ is given as:

$$
\delta \widetilde{W}_{c}=\eta \frac{E_{\infty}}{E_{0}} \sum_{j=1}^{N_{t}}\left(\prod_{p=1}^{j} \frac{p-\alpha-1}{p}\right) \times b \int_{x} \delta \mathbf{u}_{c}{ }^{T}\left[D_{c}\right]^{T}\left[C_{c}\right]\left[D_{c}\right] \widetilde{\mathbf{u}}_{c}(t-j \Delta t) d x
$$

\subsection{PFRC Laminate (Top Layer)}

The piezoelectric fiber-reinforced composite (PFRC) is identified with the subscript $t$ for the top layer. The PFRC layer acts as an actuator for the ACLD beam.

\subsubsection{Kinematic Assumptions}

\subsubsection{Mechanical Field Assumptions}

Similar to the FGM beam bottom layer, the axial displacement is linearly interpolated in the thickness coordinate and the transverse displacement is constant. The displacement vector $\bar{d}_{t}$ (includes both the axial $\bar{u}_{t}$ and transverse $\bar{w}_{t}$ displacement) is written as:

$$
\bar{d}_{t}=\left\{\begin{array}{c}
\bar{u}_{t}\left(x, z_{t}, t\right) \\
\bar{w}_{t}\left(x, z_{t}, t\right)
\end{array}\right\}=\left\{\begin{array}{c}
u_{t}(x, t)-z_{t} \phi_{t}(x, t) \\
w_{t}(x, t)
\end{array}\right\}
$$

The reference point is taken at the geometric midpoint of the ply assembly or PFRC layer. Establishing a new vector $\mathbf{u}_{t}^{T}=\left\{u_{t} w_{t} \phi_{t}\right\}$ gives the following expansion of $\bar{d}_{t}$ : 


$$
\bar{d}_{t}=\left[\begin{array}{ccc}
1 & 0 & -z_{t} \\
0 & 1 & 0
\end{array}\right]\left\{\begin{array}{c}
u_{t} \\
w_{t} \\
\phi_{t}
\end{array}\right\} \equiv\left[Z_{t}\right] \mathbf{u}_{t}
$$

The linear strain-displacement relations are:

$$
\bar{\epsilon}_{t}=\left\{\begin{array}{c}
\epsilon_{x}\left(x, z_{t}, t\right) \\
\gamma_{x z}\left(x, z_{t}, t\right)
\end{array}\right\}_{t}=\left\{\begin{array}{c}
\frac{\partial \bar{u}_{t}}{\partial x} \\
\frac{\partial \bar{u}_{t}}{\partial z_{t}}+\frac{\partial \bar{w}_{t}}{\partial x}
\end{array}\right\}=\left\{\begin{array}{c}
\frac{\partial u_{t}}{\partial x}(x, t)-z_{t} \frac{\partial \phi_{t}}{\partial x}(x, t) \\
-\phi_{t}(x, t)+\frac{\partial w_{t}}{\partial x}(x, t)
\end{array}\right\}
$$

The strain vector $\bar{\epsilon}_{t}$ can be written in the following form as:

$$
\begin{aligned}
\bar{\epsilon}_{t}=\left\{\begin{array}{c}
\epsilon_{x} \\
\gamma_{x z}
\end{array}\right\}_{t} & =\left\{\begin{array}{c}
\frac{\partial u_{t}}{\partial x}(x, t) \\
-\phi_{t}(x, t)+\frac{\partial w_{t}}{\partial x}(x, t)
\end{array}\right\}-z_{t}\left\{\begin{array}{c}
\frac{\partial \phi_{t}}{\partial x}(x, t) \\
0
\end{array}\right\} \\
& =\left\{\begin{array}{c}
\epsilon_{x}^{0} \\
\gamma_{x z}^{0}
\end{array}\right\}_{t}+z_{t}\left\{\begin{array}{c}
\kappa_{x} \\
\kappa_{x z}
\end{array}\right\}_{t} \equiv \epsilon_{0}+z_{t} \kappa
\end{aligned}
$$

Hence,

$$
\bar{\epsilon}_{t}=\left[\begin{array}{ll}
1 & z_{t}
\end{array}\right]\left\{\begin{array}{c}
\epsilon_{0} \\
\kappa
\end{array}\right\}_{t}=\left[\begin{array}{ll}
1 & z_{t}
\end{array}\right]\left[\begin{array}{c}
D_{t}^{0} \\
D_{t}^{1}
\end{array}\right] \mathbf{u}_{t}
$$

where the derivative operator matrices $\left[D_{t}^{0}\right]$ and $\left[D_{t}^{1}\right]$ are given as:

$$
\left[D_{t}^{0}\right]=\left[\begin{array}{ccc}
\frac{\partial}{\partial x} & 0 & 0 \\
0 & 0 & \frac{\partial}{\partial x}
\end{array}\right] \quad\left[D_{t}^{1}\right]=\left[\begin{array}{ccc}
0 & \frac{\partial}{\partial x} & 1 \\
0 & 0 & 0
\end{array}\right]
$$

\subsubsection{Electrical Field Assumptions}

Two assumptions are made with respect to the electrostatic field in the PFRC laminate. The first assumption is that the electrical potential variable $\psi_{t}$ is linear throughout the thickness of the top layer and is written as:

$$
\psi_{t}\left(x, z_{t}, t\right)=\psi_{0}(x, t)+z_{t} \frac{\partial \psi_{t}\left(x, z_{t}, t\right)}{\partial z_{t}}
$$

where $\psi_{0}$ and $\frac{\partial \psi_{t}}{\partial z_{t}}$ are the electric potential and its gradient at the mid-plane of the PFRC laminate, respectively. 
The second assumption is that the axial component of the electrical field (i.e. $E_{x}=0$ ) is negligible since the contribution to the electromechanical energy is insignificant compared to the transverse displacement. In addition, the voltage is assumed to be uniformly distributed along the $\mathrm{z}$ direction (thickness) because the PFRC laminate is thin.

The transverse constant electrical field $E_{z}$ can then be expressed as:

$$
E_{z}=-\frac{\partial \psi_{t}}{\partial z_{t}}=-\frac{V}{h_{t}}
$$

where $\mathrm{V}$ is the applied voltage and $h_{t}$ is the PFRC laminate thickness.

\subsubsection{Constitutive Equations}

The piezoelectric fibers in the PFRC are continuous and unidirectional and subjected to a constant electric field transverse to the fibers. The matrix and fibers have identical electric field. Further, the matrix is not piezolectrically active [20].

\subsubsection{Mechanical Properties}

The piezoelectric fibers are isotropic and lie in the transverse 1-2 plane. The constitutive relation for the $k^{\text {th }}$ ply of the PFRC in the principal 1-2-3 directions is given as:

$$
\left\{\begin{array}{c}
\sigma_{1} \\
\sigma_{2} \\
\sigma_{3} \\
\tau_{23} \\
\tau_{13} \\
\tau_{12}
\end{array}\right\}_{k}=\left[\begin{array}{cccccc}
c_{11} & c_{12} & c_{13} & 0 & 0 & 0 \\
c_{12} & c_{22} & c_{23} & 0 & 0 & 0 \\
c_{13} & c_{23} & c_{33} & 0 & 0 & 0 \\
0 & 0 & 0 & c_{44} & 0 & 0 \\
0 & 0 & 0 & 0 & c_{55} & 0 \\
0 & 0 & 0 & 0 & 0 & c_{66}
\end{array}\right]_{k}\left\{\begin{array}{c}
\epsilon_{1} \\
\epsilon_{2} \\
\epsilon_{3} \\
\gamma_{23} \\
\gamma_{13} \\
\gamma_{12}
\end{array}\right\}_{k}-\left[\begin{array}{ccc}
0 & 0 & e_{31} \\
0 & 0 & e_{31} \\
0 & 0 & e_{31} \\
0 & e_{15} & 0 \\
e_{15} & 0 & 0 \\
0 & 0 & 0
\end{array}\right]_{k}\left\{\begin{array}{c}
E_{1} \\
E_{2} \\
E_{3}
\end{array}\right\}_{k}
$$

where $i, j=1,2,3, \sigma_{i}$ and $\tau_{i j}$ are the normal and shear stress, and $\epsilon_{i}$ and $\gamma_{i j}$ are the normal and shear strain components. Also, $c_{i j}$ 's are the effective elastic coefficients determined from the micromechanical analysis at constant electric field [20]. The constants $e_{i j}$ represent the piezoelectric coefficients and $E_{i}$ is the electric field.

The 1-2-3 coordinate system is transformed to a global $x y z$ system via a counterclockwise rotation by an angle $\theta$ about the $z$ axis using the mechanical component of the transformation 
matrix:

$$
[T]=\left[\begin{array}{cccccc}
\cos ^{2} \theta & \sin ^{2} \theta & 0 & 0 & 0 & 2 \sin \theta \cos \theta \\
\sin ^{2} \theta & \cos ^{2} \theta & 0 & 0 & 0 & -2 \sin \theta \cos \theta \\
0 & 0 & 1 & 0 & 0 & 0 \\
0 & 0 & 0 & \cos \theta & -\sin \theta & 0 \\
0 & 0 & 0 & \sin \theta & \cos \theta & 0 \\
-\sin \theta \cos \theta & \sin \theta \cos \theta & 0 & 0 & 0 & \cos ^{2} \theta-\sin ^{2} \theta
\end{array}\right]
$$

Likewise, for the electro-mechanical component in Eq. 3.58, the transformation matrix is:

$$
[T]^{e}=\left[\begin{array}{ccc}
\cos \theta & -\sin \theta & 0 \\
\sin \theta & \cos \theta & 0 \\
0 & 0 & 1
\end{array}\right]
$$

Therefore, the stress-strain relation for the mechanical portion with respect to the global coordinate system is:

$$
\left\{\begin{array}{c}
\sigma_{x} \\
\sigma_{y} \\
\sigma_{z} \\
\tau_{y z} \\
\tau_{x z} \\
\tau_{x y}
\end{array}\right\}_{k}^{m}=[T]_{k}^{-1}\left[C_{t}\right]_{k}[R][T]_{k}[R]^{-1}\left\{\begin{array}{c}
\epsilon_{x} \\
\epsilon_{y} \\
\epsilon_{z} \\
\gamma_{y z} \\
\gamma_{x z} \\
\gamma_{x y}
\end{array}\right\}_{k} \equiv\left[Q_{t}\right]_{k}\left\{\begin{array}{c}
\epsilon_{x} \\
\epsilon_{y} \\
\epsilon_{z} \\
\gamma_{y z} \\
\gamma_{x z} \\
\gamma_{x y}
\end{array}\right\}_{k}
$$

where Reuter matrix $[R]$ is utilized to attribute the relationship between the tensor shear strain $\epsilon$ and the engineering shear strain $\gamma$ shown as follows:

$$
[R]=\left\{\begin{array}{llllll}
1 & 0 & 0 & 0 & 0 & 0 \\
0 & 1 & 0 & 0 & 0 & 0 \\
0 & 0 & 1 & 0 & 0 & 0 \\
0 & 0 & 0 & 2 & 0 & 0 \\
0 & 0 & 0 & 0 & 2 & 0 \\
0 & 0 & 0 & 0 & 0 & 2
\end{array}\right\}
$$

where $\sigma_{x}, \sigma_{x}$, and $\sigma_{x}$ are the normal stresses in the $\mathrm{x}, \mathrm{y}$, and $\mathrm{z}$ directions, respectively; $\tau_{x z}$ and $\tau_{y z}$ are the transverse shear stresses and $\tau_{x y}$ is the in-plane shear stress. The corresponding 
stains are $\epsilon_{x}, \epsilon_{y}, \epsilon_{z}, \gamma_{y z}, \gamma_{x z}$, and $\gamma_{x y}$. The stiffness matrix from Eq. $3.58\left[C_{t}\right]_{k}$ is written as:

$$
\left[C_{t}\right]_{k}=\left[\begin{array}{cccccc}
c_{11} & c_{12} & c_{13} & 0 & 0 & 0 \\
c_{12} & c_{22} & c_{23} & 0 & 0 & 0 \\
c_{13} & c_{23} & c_{33} & 0 & 0 & 0 \\
0 & 0 & 0 & c_{44} & 0 & 0 \\
0 & 0 & 0 & 0 & c_{55} & 0 \\
0 & 0 & 0 & 0 & 0 & c_{66}
\end{array}\right]_{k}
$$

Similarly, the stress and electric field relation of Eq. 3.58 is transformed through the following expression:

$$
\left\{\begin{array}{c}
\sigma_{x} \\
\sigma_{y} \\
\sigma_{z} \\
\tau_{y z} \\
\tau_{x z} \\
\tau_{x y}
\end{array}\right\}_{k}^{e}=[T]_{k}^{-1}[\epsilon]_{k}^{T}[T]_{k}^{e}\left\{\begin{array}{c}
E_{x} \\
E_{y} \\
E_{z}
\end{array}\right\}_{k} \equiv[e]_{k}^{T}\left\{\begin{array}{c}
E_{x} \\
E_{y} \\
E_{z}
\end{array}\right\}_{k}
$$

where the piezoelectric coefficient matrix $[e]_{k}^{T}$ is the transpose of $[e]_{k}$ and is expressed as:

$$
[e]_{k}^{T}=\left[\begin{array}{cccccc}
0 & 0 & 0 & 0 & e_{15} & 0 \\
0 & 0 & 0 & e_{15} & 0 & 0 \\
e_{31} & e_{31} & e_{33} & 0 & 0 & 0
\end{array}\right]_{k}
$$

The final constitutive relation is derived following the work by Sun and Huang [21]. The stress and strain about the $\mathrm{x}$ direction are assumed to be zero (i.e. $\sigma_{z}=\epsilon_{z}=0$ ) since the thickness of the PFRC layer is negligible. Applying these changes, the updated constitutive equation is expressed as:

$$
\left\{\begin{array}{c}
\sigma_{x} \\
\sigma_{y} \\
\tau_{y z} \\
\tau_{x z} \\
\tau_{x y}
\end{array}\right\}_{k}=\left[\begin{array}{ccccc}
\bar{Q}_{11} & \bar{Q}_{12} & 0 & 0 & \bar{Q}_{16} \\
\bar{Q}_{12} & \bar{Q}_{22} & 0 & 0 & \bar{Q}_{26} \\
0 & 0 & \bar{Q}_{44} & \bar{Q}_{45} & 0 \\
0 & 0 & \bar{Q}_{45} & \bar{Q}_{55} & 0 \\
\bar{Q}_{16} & \bar{Q}_{26} & 0 & 0 & \bar{Q}_{66}
\end{array}\right]_{k}\left\{\begin{array}{c}
\epsilon_{x} \\
\epsilon_{y} \\
\gamma_{y z} \\
\gamma_{x z} \\
\gamma_{x y}
\end{array}\right\}_{k}-\left[\begin{array}{ccc}
0 & 0 & \bar{e}_{31} \\
0 & 0 & \bar{e}_{31} \\
-\bar{e}_{25} & \bar{e}_{15} & 0 \\
\bar{e}_{15} & \bar{e}_{25} & 0 \\
0 & 0 & 0
\end{array}\right]_{k}\left\{\begin{array}{l}
E_{x} \\
E_{y} \\
E_{z}
\end{array}\right\}_{k}
$$


where $\bar{e}_{31}=e_{3} 1, \bar{e}_{15}=e_{15}\left(\cos ^{2} \theta-\sin ^{2} \theta\right)$ and $\bar{e}_{25}=-2 e_{15} \sin \theta \cos \theta$, and

$$
\begin{aligned}
& \bar{Q}_{11}=c_{11} \cos ^{4} \theta+2 c_{12} \sin ^{2} \theta \cos ^{2} \theta+c_{22} \sin ^{4} \theta+4 c_{66} \sin ^{2} \theta \cos ^{2} \theta \\
& \bar{Q}_{12}=c_{11} \sin ^{2} \theta \cos ^{2} \theta+c_{12}\left(\sin ^{4} \theta+\cos ^{4} \theta\right)+c_{22} \sin ^{2} \theta \cos ^{2} \theta-4 c_{66} \sin ^{2} \theta \cos ^{2} \theta \\
& \bar{Q}_{16}=\left[c_{11} \cos ^{2} \theta+c_{12}\left(\sin ^{2} \theta-\cos ^{2} \theta\right)-c_{22} \sin ^{2} \theta+2 c_{66}\left(\sin ^{2} \theta-\cos ^{2} \theta\right)\right] \sin \theta \cos \theta \\
& \bar{Q}_{22}=c_{11} \sin ^{4} \theta+2 c_{12} \sin ^{2} \theta \cos ^{2} \theta+c_{22} \cos ^{4} \theta+4 c_{66} \sin ^{2} \theta \cos ^{2} \theta \\
& \bar{Q}_{26}=\left[c_{11} \sin ^{2} \theta-c_{12}\left(\sin ^{2} \theta-\cos ^{2} \theta\right)-c_{22} \cos ^{2} \theta-2 c_{66}\left(\sin ^{2} \theta-\cos ^{2} \theta\right)\right] \sin \theta \cos \theta \\
& \bar{Q}_{44}=c_{44} \cos ^{2} \theta+c_{55} \sin ^{2} \theta \\
& \bar{Q}_{45}=-c_{44} \sin \theta \cos \theta+c_{55} \sin \theta \cos \theta \\
& \bar{Q}_{55}=c_{44} \sin ^{2} \theta+c_{55} \cos ^{2} \theta \\
& \bar{Q}_{66}=c_{11} \sin ^{2} \theta \cos ^{2} \theta-2 c_{12} \sin ^{2} \theta \cos ^{2} \theta+c_{22} \sin ^{2} \theta \cos ^{2} \theta+c_{66}\left(\sin ^{4} \theta-2 \sin ^{2} \theta \cos ^{2} \theta+\operatorname{coss}^{4} \theta\right)
\end{aligned}
$$

Assuming also that $\sigma_{y}=\tau_{y z}=\tau_{x y}=0$ albeit $\epsilon_{y} \neq \gamma_{y z} \neq \gamma_{x y} \neq 0$, and noting that $E_{x}=E_{y}=0$ because the piezoelectric fibers are polarized only through the thickness, the final constitutive equation is reduced to:

$$
\begin{aligned}
\left\{\bar{\sigma}_{t}\right\}_{k}=\left\{\begin{array}{c}
\sigma_{x} \\
\tau_{x z}
\end{array}\right\}_{k} & =\left[\begin{array}{cc}
\bar{Q}_{11} & 0 \\
0 & k_{s} \bar{Q}_{55}
\end{array}\right]_{k}\left\{\begin{array}{c}
\epsilon_{x} \\
\gamma_{x z}
\end{array}\right\}_{k}-\left\{\begin{array}{c}
\bar{e}_{31} E_{z} \\
0
\end{array}\right\}_{k} \\
& \equiv\left[\bar{Q}_{t}\right]_{k}\left\{\epsilon_{t}\right\}_{k}-\left\{\begin{array}{c}
\bar{e}_{31} E_{z} \\
0
\end{array}\right\}_{k}
\end{aligned}
$$

where,

$$
\begin{aligned}
& \widetilde{Q}_{11}=\bar{Q}_{11}+\frac{\bar{Q}_{16} \bar{Q}_{26}-\bar{Q}_{12} \bar{Q}_{66}}{\bar{Q}_{22} \bar{Q}_{66}-\bar{Q}_{26}^{2}} \bar{Q}_{12}+\frac{\bar{Q}_{12} \bar{Q}_{26}-\bar{Q}_{16} \bar{Q}_{22}}{\bar{Q}_{22} \bar{Q}_{66}-\bar{Q}_{26}^{2}} \bar{Q}_{16}, \\
& \widetilde{Q}_{55}=\bar{Q}_{55}-\frac{\bar{Q}_{45}^{2}}{\bar{Q}_{44}}, \quad \widetilde{e}_{31}=\left(1-\frac{\bar{Q}_{16} \bar{Q}_{26}-\bar{Q}_{12} \bar{Q}_{66}}{\bar{Q}_{22} \bar{Q}_{66}-\bar{Q}_{26}^{2}}\right) \bar{e}_{31},
\end{aligned}
$$

and $k_{s}=5 / 6$ is the shear correction factor.

\subsubsection{Electrical Properties}

The direct piezoelectric effect would be considered when one wants to model a self-sensing actuator. For completeness, the electrical displacement field $D_{i}$ is given by [22] and is 
expressed in the 1-2-3 principle directions as:

$$
\left\{\begin{array}{c}
D_{1} \\
D_{2} \\
D_{3}
\end{array}\right\}_{k}=\left[\begin{array}{cccccc}
0 & 0 & 0 & 0 & e_{15} & 0 \\
0 & 0 & 0 & e_{15} & 0 & 0 \\
e_{31} & e_{31} & e_{33} & 0 & 0 & 0
\end{array}\right]_{k}\left\{\begin{array}{c}
\epsilon_{1} \\
\epsilon_{2} \\
\epsilon_{3} \\
\gamma_{23} \\
\gamma_{13} \\
\gamma_{12}
\end{array}\right\}_{k}+\left[\begin{array}{ccc}
d_{11} & 0 & 0 \\
0 & d_{22} & 0 \\
0 & 0 & d_{33}
\end{array}\right]_{k}\left\{\begin{array}{c}
0 \\
0 \\
E_{z}
\end{array}\right\}_{k}
$$

where the coefficients $d_{i j}$ are the components of the dielectric permittivity tensor at constant strain.

The permittivity constant matrix $\left[d_{k}\right]=\left[\begin{array}{ccc}d_{11} & 0 & 0 \\ 0 & d_{22} & 0 \\ 0 & 0 & d_{33}\end{array}\right]_{k}$ is transformed to determine the modified electrical displacement relation in the xyz coordinate system as follows:

$$
\begin{aligned}
\left\{\begin{array}{c}
D_{x} \\
D_{y} \\
D_{z}
\end{array}\right\}_{e}^{m} & =[T]_{k}^{-1}[e]_{k}^{T}[T]_{k}^{e}\left\{\begin{array}{c}
\epsilon_{x} \\
\epsilon_{y} \\
\epsilon_{z} \\
\gamma_{y z} \\
\gamma_{x z} \\
\gamma_{x y}
\end{array}\right\}_{k}+[T]_{k}^{-1}[d]_{k}^{T}[T]_{k}^{e}\left\{\begin{array}{c}
0 \\
0 \\
E_{z}
\end{array}\right\}_{k} \\
& \equiv[\bar{e}]_{k}\left\{\begin{array}{c}
\epsilon_{x} \\
\epsilon_{y} \\
\epsilon_{z} \\
\gamma_{y z} \\
\gamma_{x z} \\
\gamma_{x y}
\end{array}\right\}_{k}+[\bar{d}]_{k}\left\{\begin{array}{c}
0 \\
0 \\
E_{z}
\end{array}\right\}_{k}
\end{aligned}
$$

With the assumption that $\epsilon_{z}=0$, the constitutive electrical displacement relation is updated to:

$$
\left\{\begin{array}{l}
D_{x} \\
D_{y} \\
D_{z}
\end{array}\right\}_{k}=\left[\begin{array}{cccccc}
0 & 0 & 0 & -\bar{e}_{25} & e_{15} & 0 \\
0 & 0 & 0 & e_{15} & \bar{e}_{25} & 0 \\
e_{31} & e_{31} & 0 & 0 & 0 & 0
\end{array}\right]_{k}\left\{\begin{array}{c}
\epsilon_{x} \\
\epsilon_{y} \\
\gamma_{y z} \\
\gamma_{x z} \\
\gamma_{x y}
\end{array}\right\}_{k}+\left[\begin{array}{ccc}
\bar{d}_{11} & \bar{d}_{12} & 0 \\
\bar{d}_{12} & \bar{d}_{22} & 0 \\
0 & 0 & \bar{d}_{33}
\end{array}\right]_{k}\left\{\begin{array}{c}
0 \\
0 \\
E_{z}
\end{array}\right\}_{k}
$$


where $\bar{d}_{11}=d_{11} \cos ^{2} \theta+d_{22} \sin ^{2} \theta, \bar{d}_{12}=\left(d_{22}-d_{11}\right) \sin \theta \cos \theta, \bar{d}_{22}=d_{11} \sin ^{2} \theta+d_{22} \cos ^{2} \theta$, and $\bar{d}_{33}=d_{33}$. Assuming also that $d_{x}^{k}=D_{y}^{k}=0$, the z direction electrical displacement field $D_{z}^{k}$ reduces to:

$$
D_{z}^{k}=\bar{e}_{31} \epsilon_{x}+\bar{e}_{31} \epsilon_{y}+\bar{d}_{33} E_{z}^{k}
$$

The enforcement of the condition $\sigma_{y}=\gamma_{y z}=\gamma_{x y}=0$ from beam theory further reduces the constitutive relation to:

$$
D_{z}^{k}=\widetilde{e}_{31} \epsilon_{x}+\widetilde{d}_{33} E_{z}^{k}
$$

where $\widetilde{d}_{33}=\frac{\bar{Q}_{66} \bar{e}_{31}^{2}}{\bar{Q}_{22} \bar{Q}_{66}-\bar{Q}_{26}^{2}}+\bar{d}_{33}$.

\subsubsection{Laminate Stiffness Matrix ABD}

Applying the classical laminate theory (CLT) [23, 24], the resultant laminate stiffness matrix $\mathrm{ABD}$ can be determined. The force and moment resultants in the $\mathrm{x}$ coordinate are first determined with respect to the reference plane, at the middle of the laminate. The geometry of a laminate with $n$ layers is shown in Figure 3.2 .

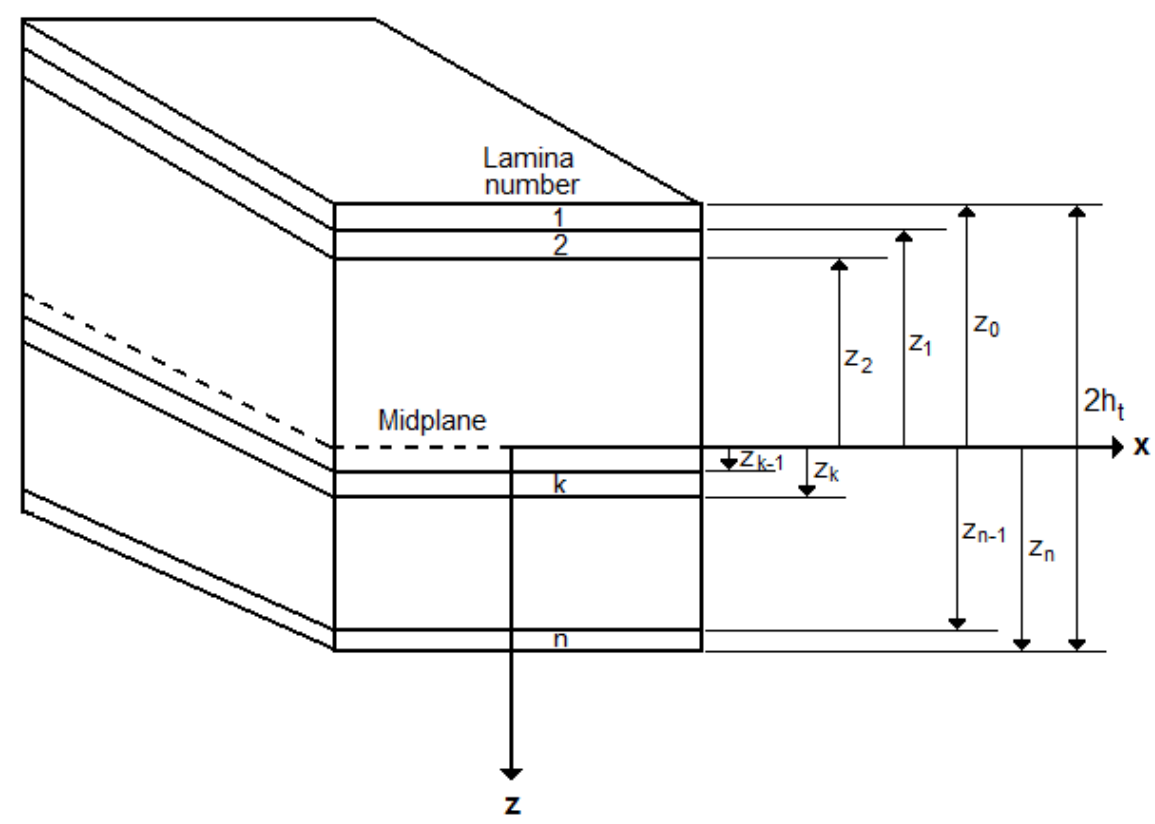

Figure 3.2: Geometry of an $n$-layered laminate. [23]

The resultant forces and moments acting on the laminate are determined by integrating the stresses and moments of the stresses at each ply through the thickness of the laminate 
[23, 24]. The normal force resultant $N_{x}$ and shear force resultant $N_{x z}$ as well as the bending moment resultant $M_{x}$ and the twisting moment resultant $M_{x z}$ are shown below:

$$
\begin{gathered}
\left\{\begin{array}{c}
N_{x} \\
N_{x z}
\end{array}\right\}=\int_{-h_{t}}^{h_{t}}\left\{\begin{array}{c}
\sigma_{x} \\
\tau_{x z}
\end{array}\right\} d z=\sum_{k=1}^{n} \int_{z_{k-1}}^{z_{k}}\left\{\begin{array}{c}
\sigma_{x} \\
\tau_{x z}
\end{array}\right\}_{k} d z \\
\left\{\begin{array}{c}
M_{x} \\
M_{x z}
\end{array}\right\}=\int_{-h_{t}}^{h_{t}}\left\{\begin{array}{c}
\sigma_{x} \\
\tau_{x z}
\end{array}\right\} z d z=\sum_{k=1}^{n} \int_{z_{k-1}}^{z_{k}}\left\{\begin{array}{c}
\sigma_{x} \\
\tau_{x z}
\end{array}\right\}_{k} z d z
\end{gathered}
$$

where the stress resultants $N_{x}, N_{x z}$ are depicted as force per unit length and the moment resultants $M_{x}, M_{x z}$ are depicted as moment per unit length in the x direction.

Taking the stiffness matrix from Eq. 3.64 and the strain vector from Eq. 3.55, the force and moment resultants can be expressed in the following matrix form [23, 24]:

$$
\begin{aligned}
& \left\{\begin{array}{l}
N_{x} \\
N_{x z}
\end{array}\right\}=\left[\begin{array}{ll}
A_{11} & A_{12} \\
A_{12} & A_{22}
\end{array}\right]\left\{\begin{array}{c}
\epsilon_{x}^{0} \\
\gamma_{x z}^{0}
\end{array}\right\}+\left[\begin{array}{ll}
B_{11} & B_{12} \\
B_{12} & B_{22}
\end{array}\right]\left\{\begin{array}{c}
\kappa_{x} \\
\kappa_{x z}
\end{array}\right\}-\left\{\begin{array}{c}
N_{x} \\
0
\end{array}\right\}^{t} \\
& \left\{\begin{array}{l}
M_{x} \\
M_{x z}
\end{array}\right\}=\left[\begin{array}{ll}
B_{11} & B_{12} \\
B_{12} & B_{22}
\end{array}\right]\left\{\begin{array}{c}
\epsilon_{x}^{0} \\
\gamma_{x z}^{0}
\end{array}\right\}+\left[\begin{array}{ll}
D_{11} & D_{12} \\
D_{12} & D_{22}
\end{array}\right]\left\{\begin{array}{c}
\kappa_{x} \\
\kappa_{x z}
\end{array}\right\}-\left\{\begin{array}{c}
M_{x} \\
0
\end{array}\right\}^{t}
\end{aligned}
$$

where

$$
\begin{aligned}
& A_{i j}=\sum_{k=1}^{n}\left(\widetilde{Q}_{t_{i j}}\right)_{k}\left(z_{k}-z_{k-1}\right) \\
& B_{i j}=\frac{1}{2} \sum_{k=1}^{n}\left(\widetilde{Q}_{t_{i j}}\right)_{k}\left(z_{k}^{2}-z_{k-1}^{2}\right) \\
& D_{i j}=\frac{1}{3} \sum_{k=1}^{n}\left(\widetilde{Q}_{t_{i j}}\right)_{k}\left(z_{k}^{3}-z_{k-1}^{3}\right)
\end{aligned}
$$

and

$$
\begin{aligned}
N_{x}^{t} & =\sum_{k=1}^{n}\left(\widetilde{e}_{31} E_{z}\right)_{k}\left(z_{k}-z_{k-1}\right) \\
M_{x}^{t} & =\frac{1}{2} \sum_{k=1}^{n}\left(\widetilde{e}_{31} E_{z}\right)_{k}\left(z_{k}^{2}-z_{k-1}^{2}\right)
\end{aligned}
$$


The laminate stiffness matrices so-called ABD matrix includes the extensional stiffnesses $A_{i j}$, the coupling stiffnesses $B_{i j}$, and the bending stiffness $D_{i j} . N_{x}^{t}$ and $M_{x}^{t}$ are components of the electrical stress resultant.

\subsubsection{Variational Formulation using Hamilton's principle}

\subsubsection{Kinetic Energy}

Analogous to the FGM beam and viscoelastic layer, the variation of kinetic energy for the PFRC laminate is:

$$
\delta T_{t}=\int_{v} \rho_{t} \delta \bar{d}_{t}^{T} \ddot{\bar{d}}_{t} d v
$$

Substituting for $\bar{d}_{t}$ from Eq. 3.52 yields:

$$
\begin{aligned}
\delta T_{t} & =\rho_{t} \int_{x} \int_{y} \delta \mathbf{u}_{t}^{T}\left[I_{t}\right] \ddot{\mathbf{u}}_{t} d y d x \\
& =\rho_{t} b \int_{x} \delta \mathbf{u}_{t}^{T}\left[I_{t}\right] \ddot{\mathbf{u}}_{t} d x
\end{aligned}
$$

where $\left[I_{t}\right]$ is expressed as:

$$
\left[I_{t}\right]=\sum_{k=1}^{n} \int_{z_{k}}\left(\left[Z_{t}\right]^{T}\left[Z_{t}\right]\right) d z=\sum_{k=1}^{n} \int_{z_{k}}\left[\begin{array}{ccc}
1 & 0 & -z_{t} \\
0 & 1 & 0 \\
-z_{t} & 0 & z_{t}^{2}
\end{array}\right] d z
$$

\subsubsection{Strain Energy}

The total variational strain energy for the PFRC laminate is described as:

$$
\delta U_{t}=\delta U_{t}^{M}+\delta W_{t}^{E}
$$

where $U_{t}^{M}$ represents the mechanical strain energy and $W_{t}^{E}$ is the work done by the electrical force created from the applied electric filed $E_{z}$.

The variation of the total mechanical strain energy for the PFRC laminate is as follows:

$$
\delta U_{t}^{M}=\int_{v} \delta \bar{\epsilon}_{t}^{T} \bar{\sigma}_{t} d v
$$

Using the stress-strain relation of each ply in the lamina $\left\{\bar{\sigma}_{t}\right\}_{k}=\left[\widetilde{Q}_{t}\right]_{k} \bar{\epsilon}_{t}$ from Eq. 3.64. the 
variational mechanical strain can be expanded as:

$$
\delta U_{t}=\int_{x} \int_{y} \sum_{k=1}^{n} \int_{z_{k-1}}^{z_{k}} \delta \bar{\epsilon}_{t}^{T}\left[\widetilde{Q}_{t}\right]_{k} \delta \bar{\epsilon}_{t} d z d y d x
$$

Substituting the strain vector from Eq. 3.55 into the above strain energy equation gives:

$$
\begin{aligned}
\delta U_{t} & =\int_{x} \int_{y} \sum_{k=1}^{n} \int_{z_{k-1}}^{z_{k}}\left\{\begin{array}{c}
\delta \epsilon_{0} \\
\delta \kappa
\end{array}\right\}_{t}^{T}\left[\begin{array}{cc}
\widetilde{Q}_{t} & z_{t} \widetilde{Q}_{t} \\
z_{t} \widetilde{Q}_{t} & z_{t}^{2} \widetilde{Q}_{t}
\end{array}\right]_{k}\left\{\begin{array}{c}
\epsilon_{0} \\
\kappa
\end{array}\right\}_{t} d z d y d x \\
& \equiv b \int_{x}\left\{\begin{array}{c}
\delta \epsilon_{0} \\
\delta \kappa
\end{array}\right\}_{t}\left[\begin{array}{ll}
A & B \\
B & D
\end{array}\right]\left\{\begin{array}{c}
\epsilon_{0} \\
\kappa
\end{array}\right\}_{t} d x
\end{aligned}
$$

where the ABD laminate stiffness matrices are found using Eq. 3.74 and gives:

$$
A=\left[\begin{array}{ll}
A_{11} & A_{12} \\
A_{12} & A_{22}
\end{array}\right], \quad B=\left[\begin{array}{ll}
B_{11} & B_{12} \\
B_{12} & B_{22}
\end{array}\right], \quad D=\left[\begin{array}{cc}
D_{11} & D_{12} \\
D_{12} & D_{22}
\end{array}\right]
$$

The final expression of the variational mechanical strain energy is found by substituting $\left\{\begin{array}{c}\epsilon_{0} \\ \kappa\end{array}\right\}_{t}=\left[\begin{array}{c}D_{t}^{0} \\ D_{t}^{1}\end{array}\right] \mathbf{u}_{t}$ from Eq. 3.55 shown below:

$$
\delta U_{t}^{M}=b \int_{x} \delta \mathbf{u}_{t}^{T}\left[\begin{array}{c}
D_{t}^{0} \\
D_{t}^{1}
\end{array}\right]^{T}\left[\begin{array}{cc}
A & B \\
B & D
\end{array}\right]\left[\begin{array}{c}
D_{t}^{0} \\
D_{t}^{1}
\end{array}\right] \mathbf{u}_{t} d x
$$

The construction of the actuator is found by applying an electric field to the PFRC laminate, where the electric field is found via a simple proportional-derivative (PD) controller. This electric field is determined by the PD controllers' derivative constant [25]. A velocity feedback control gain is used to signal the actuator.

Considering the electrical portion of the strain energy, the virtual work $\delta W_{t}^{E}$ of the PFRC can be written as:

$$
\delta W_{t}^{E}=-\left(\int_{v} \delta \bar{\epsilon}_{t}^{T} \bar{E} d v+\int_{v} \bar{E}^{T}[\bar{d}]_{k}^{T} \delta \bar{E} d v\right) \equiv-\int_{v} \delta \bar{\epsilon}_{t}^{T} \bar{E} d v
$$

where $\bar{E}=\left\{\begin{array}{lll}0 & 0 & E_{z}\end{array}\right\}^{T}$. The variation of the electrical field is $\delta \bar{E}=0$ as the electrical field $\bar{E}$ is known. The mechanical portion of the virtual work is determined in the same manner as the electrical electrical part. Doing so, by replacing $\bar{\epsilon}_{t}$ with Eq. 3.55 and substituting 
$[\bar{e}]_{k}^{T} \bar{E}=\left\{\widetilde{e}_{31} E_{z}\right\}_{k}$ from Eq. 3.64 to the previous virtual work expression yields:

$$
\delta \hat{W}_{t}=-b \int_{x} \delta \mathbf{u}_{t}^{T}\left[\begin{array}{c}
D_{t}^{0} \\
D_{t}^{1}
\end{array}\right]^{T}\left\{\begin{array}{c}
N_{x}^{t} \\
0 \\
M_{x}^{t} \\
0
\end{array}\right\} d x
$$

where the electrical field resultants $N_{x}^{t}$ and $M_{x}^{t}$ are determined from Eq. 3.75 . 


\section{Chapter 4}

\section{Finite Element Modeling}

As mentioned in Chapter 1, the bottom FGM beam and top PFRC laminate are modeled using either Euler-Bernoulli beam theory or Timoshenko beam theory. The kinematics of these theories involve a linear axial displacement and a constant transverse displacement. The kinematics of the viscoelastic layer is expressed using quadratic and cubic interpolations in the transverse and axial displacements, respectively. This section presents the finite element formulation of the ACLD beam with the bottom FGM beam and top PFRC laminate represented as Timoshenko beams. The schematic of a representative finite element of the ACLD beam is shown in Figure 4.1 .

The through-the-thickness variations of the displacements are defined using five locations along the entire thickness of the system: one for the bottom FGM beam, three for the viscoelastic core, and one for the top PFRC layer. These five locations are used to capture the field variables for each layer. Three nodes are defined along the span of the beam to allow for a quadratic interpolation of the transverse displacement field variable due to Timoshenko's beam theory. Nodes 1 and 3 have the same five locations through-the-thickness displacements, while node 2 has only the transverse displacement of each layer.

The bottom FGM beam and top PFRC laminate are represented as Timoshenko beams. The axial displacements is interpolated with a linear function and the transverse displacements with a quadratic function. For the viscoelastic core, the axial displacement is represented by a cubic function, and the transverse displacement is interpolated with a quadratic function. The mixed interpolations prevent the occurrence of shear-locking.

The global displacement vector of an element can be written as:

$$
u_{e}^{T}=\left\{\begin{array}{lllllllll}
u_{b} & w_{b} & \phi_{b} & u_{c 2} & u_{c 3} & w_{c} & u_{t} & w_{t} & \phi_{t}
\end{array}\right\}
$$

where $u_{i}$ and $w_{i}$ are the axial and transverse displacement magnitudes, respectively, evaluated 


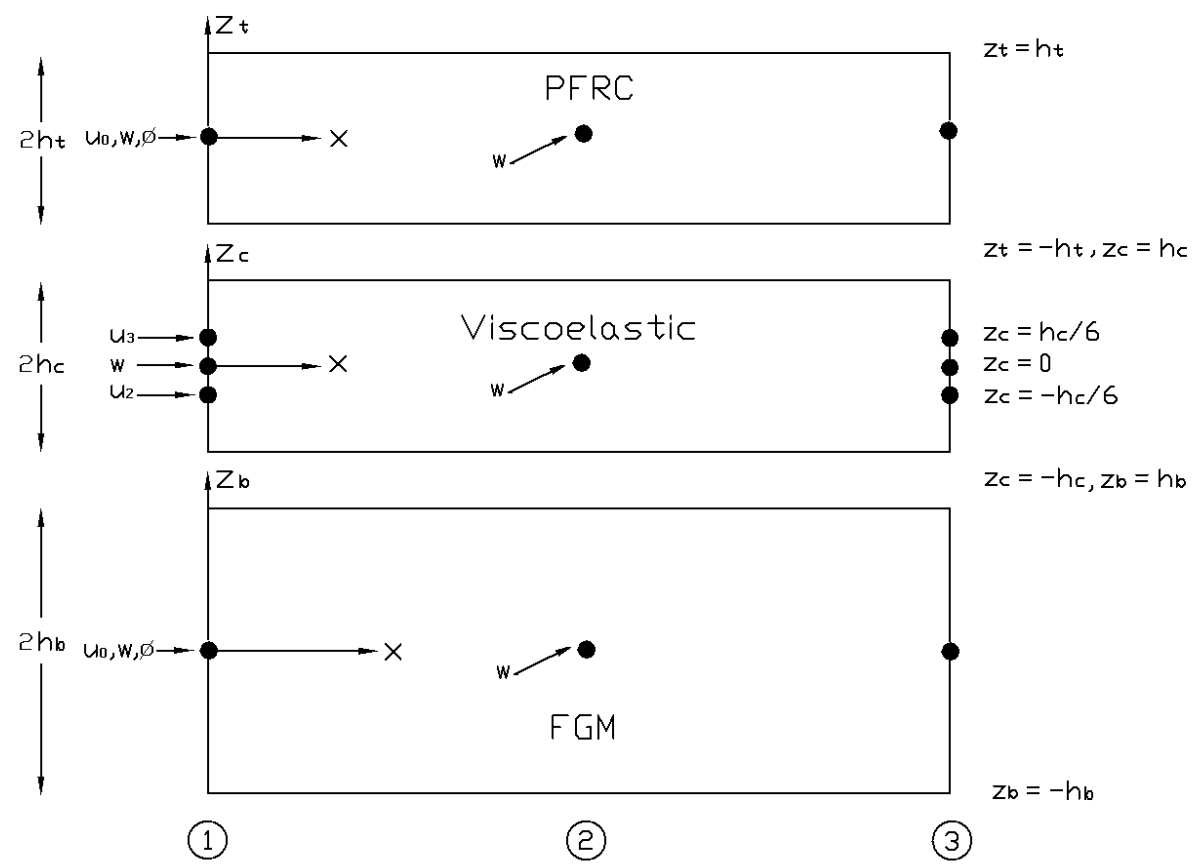

Figure 4.1: Finite element model of ACLD beam.

at $i=b, c$, and $t$ for bottom, core, and top layers, respectively. Also $u_{c 2}$ and $u_{c 3}$ are the two intermediate nodes in the core thickness.

From the finite element schematic, it is established that both the first and third nodes each contains 9 degrees of freedom (DOF), and the middle node has 3 DOF, resulting in a total elemental DOF of 21. The element displacement vector $q_{e}$ can be expressed as:

$$
\begin{aligned}
& q_{e}^{T}=\left\{\begin{array}{llllllllll}
u_{b 1} & w_{b 1} & \phi_{b 1} & u_{c 2,1} & u_{c 3,1} & w_{c 1} & u_{t 1} & w_{t 1} & \phi_{t 1} & \ldots
\end{array}\right. \\
& \begin{array}{lllll}
\ldots w_{b 2} & w_{c 2} & w_{t 2} & \ldots
\end{array} \\
& \left.\ldots u_{b 3} \quad w_{b 3} \quad \phi_{b 3} \quad u_{c 2,3} \quad u_{c 3,3} \quad w_{c 3} \quad u_{t 3} \quad w_{t 3} \quad \phi_{t 3}\right\}
\end{aligned}
$$

where the subscripts $b_{j}, c_{j}$, and $t_{j}$ represents the bottom, core, and top layer, respectively with $j=1 \ldots 3$ signifying the node numbers.

Note that if the top and bottom layers were Euler-Bernoulli beams, then $\phi=\frac{\partial w}{\partial x}$ and there would only be two element nodes along the span of the beam because the axial displacement is interpolated by linear functions and the Hermite cubic polynomial is used for the transverse displacement. 


\subsection{Shape Functions}

The axial displacement $u$ and slope $\phi$ field variables are interpolated along the span of the structure by a linear function, and transverse displacement field variable $w$ by a quadratic function. These are:

$$
\begin{aligned}
& \text { Linear Shape Functions } \\
& N_{1 \xi}=\frac{1-\xi}{2} \text { and } N_{2 \xi}=\frac{\xi+1}{2} \text { for }-1 \leq \xi \leq 1
\end{aligned}
$$

Quadratic Shape Functions

$$
N_{3 \xi}=\frac{\xi^{2}-\xi}{2} \quad N_{4 \xi}=1-\xi^{2} \quad N_{5 \xi}=\frac{\xi^{2}+\xi}{2} \text { for }-1 \leq \xi \leq 1
$$

\subsubsection{FGM Beam (Bottom Layer)}

From chapter 3, further expansion of Eq. 3.21, the kinetic energy becomes:

$$
\delta T_{b}=b \int_{x} \rho_{b} \delta u_{b}^{T} \ddot{u}_{b} d x+b \int_{x} \rho_{b} \delta w_{b}^{T} \ddot{w}_{b} d x+\alpha b \int_{x} \rho_{b} \delta \phi_{b}^{T} \ddot{\phi}_{b} d x
$$

The boolean variable $\alpha$ is 1 for Timoshenko beam theory and 0 for Euler-Bernoulli beam theory. Also, there is no shear in Euler-Bernoulli beam theory $\left(i . e . \gamma_{x z}=0\right)$, hence $\phi_{b}(x, t)=$ $\frac{\partial w_{b}}{\partial x}(x, t)$.

The displacement vector from Eq. $3.2 \mathbf{u}_{b}^{T}=\left\{u_{b} w_{b} \phi_{b}\right\}$ can be expanded as:

$$
\mathbf{u}_{b}=\left[N_{b}\right] q_{e}
$$

where

$$
\left[N_{b}\right]=\left[\begin{array}{ccccccccccccccccccccc}
N_{1 \xi} & 0 & 0 & 0 & 0 & 0 & 0 & 0 & 0 & 0 & 0 & 0 & N_{2 \xi} & 0 & 0 & 0 & 0 & 0 & 0 & 0 & 0 \\
0 & N_{3 \xi} & 0 & 0 & 0 & 0 & 0 & 0 & 0 & N_{4 \xi} & 0 & 0 & 0 & N_{5 \xi} & 0 & 0 & 0 & 0 & 0 & 0 & 0 \\
0 & 0 & N_{1 \xi} & 0 & 0 & 0 & 0 & 0 & 0 & 0 & 0 & 0 & 0 & 0 & N_{2 \xi} & 0 & 0 & 0 & 0 & 0 & 0
\end{array}\right]
$$

Subsequently, by substituting Eq. 4.9 into Eq. 3.21, the variation of the kinetic energy of the FGM beam is written as:

$$
\delta T_{b}=b \int_{-1}^{1} \rho_{b}\left(\delta q_{e}^{T}\left[N_{b}\right]^{T}\left[I_{b}\right]\left[N_{b}\right] \ddot{q}_{e}\right)|J(\xi)| d \xi=\delta q_{e}^{T}\left[M_{b}\right]^{e} \ddot{q}_{e}
$$


where $\left[M_{b}\right]^{e}=b \int_{-1}^{1} \rho_{b}\left(\left[N_{b}\right]^{T}\left[I_{b}\right]\left[N_{b}\right]\right)|J(\xi)| d \xi$ is the mass matrix of the FGM beam.

The absolute value of the Jacobian, denoted by $|J(\xi)|$, is used to transform the integration of the variables along the $x$-axis from the global coordinate $x$ (i.e. $x_{1}$ to $x_{2}$ ) to the local coordinate $\xi\left(\right.$ i.e. -1 to 1 ). The Jacobian was determined to be $|J(\xi)|=\frac{L_{e}}{2}$ in this specific case.

The same procedure is followed in the formulation of the stiffness matrix by substituting $\mathbf{u}_{b}$ from Eq. 4.6 into Eq. 3.24, expressing the variational strain energy as:

$$
\delta U_{b}=b \int_{-1}^{1}\left(\delta q_{e}^{T}\left[B_{b}\right]^{T}\left[C_{b}\right]\left[B_{b}\right] q_{e}\right)|J(\xi)| d \xi=\delta q_{e}^{T}\left[K_{b}\right]^{e} q_{e}
$$

where the stiffness matrix of the FGM beam is

$$
\begin{aligned}
& {\left[K_{b}\right]^{e}=b \int_{-1}^{1}\left(\left[B_{b}\right]^{T}\left[C_{b}\right]\left[B_{b}\right]\right)|J(\xi)| d \xi,\left[B_{b}\right]=\left[D_{b}\right]\left[N_{b}\right], \text { and }} \\
& {\left[C_{b}\right]=\int_{z}\left(\left[\widetilde{Z}_{b}\right]^{T}\left[\bar{Q}_{b}\right]\left[\widetilde{Z}_{b}\right]\right) .}
\end{aligned}
$$

\subsubsection{Viscoelastic Layer (Core)}

In shape function matrix notation, the displacement vector from Eq. 3.26 ,

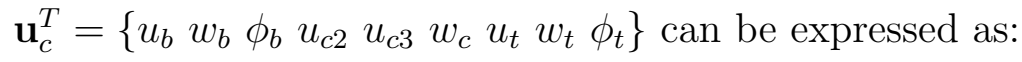

$$
\mathbf{u}_{c}=\left[N_{c}\right] q_{e}
$$

where

$$
\left[N_{c}\right]=\left[\begin{array}{ccccccccccccccccccccc}
N_{1 \xi} & 0 & 0 & 0 & 0 & 0 & 0 & 0 & 0 & 0 & 0 & 0 & N_{2 \xi} & 0 & 0 & 0 & 0 & 0 & 0 & 0 & 0 \\
0 & N_{3 \xi} & 0 & 0 & 0 & 0 & 0 & 0 & 0 & N_{4 \xi} & 0 & 0 & 0 & N_{5 \xi} & 0 & 0 & 0 & 0 & 0 & 0 & 0 \\
0 & 0 & N_{1 \xi} & 0 & 0 & 0 & 0 & 0 & 0 & 0 & 0 & 0 & 0 & 0 & N_{2 \xi} & 0 & 0 & 0 & 0 & 0 & 0 \\
0 & 0 & 0 & N_{1 \xi} & 0 & 0 & 0 & 0 & 0 & 0 & 0 & 0 & 0 & 0 & 0 & N_{2 \xi} & 0 & 0 & 0 & 0 & 0 \\
0 & 0 & 0 & 0 & N_{1 \xi} & 0 & 0 & 0 & 0 & 0 & 0 & 0 & 0 & 0 & 0 & 0 & N_{2 \xi} & 0 & 0 & 0 & 0 \\
0 & 0 & 0 & 0 & 0 & N_{3 \xi} & 0 & 0 & 0 & 0 & N_{4 \xi} & 0 & 0 & 0 & 0 & 0 & 0 & N_{5 \xi} & 0 & 0 & 0 \\
0 & 0 & 0 & 0 & 0 & 0 & N_{1 \xi} & 0 & 0 & 0 & 0 & 0 & 0 & 0 & 0 & 0 & 0 & 0 & N_{2 \xi} & 0 & 0 \\
0 & 0 & 0 & 0 & 0 & 0 & 0 & N_{3 \xi} & 0 & 0 & 0 & N_{4 \xi} & 0 & 0 & 0 & 0 & 0 & 0 & 0 & N_{5 \xi} & 0 \\
0 & 0 & 0 & 0 & 0 & 0 & 0 & 0 & N_{1 \xi} & 0 & 0 & 0 & 0 & 0 & 0 & 0 & 0 & 0 & 0 & 0 & N_{2 \xi}
\end{array}\right]
$$


In a similar manner, the anelastic displacement vector $\widetilde{\mathbf{u}}_{c}(t)$ can be expressed as:

$$
\widetilde{\mathbf{u}}_{c} \equiv\left[N_{c}\right] \widetilde{q}_{e}
$$

Substituting Eq. 4.10 at a give time $t$ into Eq. 3.39, $\widetilde{q}_{e}(t)$ can be expressed as:

$$
\widetilde{q}_{c}(t)=(1-\eta) \frac{E_{\infty}-E_{0}}{E_{\infty}} \bar{q}_{e}(t)-\eta \sum_{j=1}^{N_{t}}\left(\prod_{p=1}^{j} \frac{p-\alpha-1}{p}\right) \widetilde{q}_{e}(t-j \Delta t)
$$

Furthermore, by substituting $\mathbf{u}_{c}$ from Eq. 4.9 into Eq. 3.44, the variational kinetic energy of the viscoelastic core can be expressed, in its final form, as:

$$
\delta T_{c}=\rho_{c} b \int_{-1}^{1}\left(\delta q_{e}^{T}\left[N_{c}\right]^{T}\left[I_{c}\right]\left[N_{c}\right] \ddot{q}_{e}\right)|J(\xi)| d \xi=\delta q_{e}^{T}\left[M_{c}\right]^{e} \ddot{q}_{e}
$$

where $\left[M_{c}\right]^{e}=\rho_{c} b \int_{-1}^{1}\left(\left[N_{c}\right]^{T}\left[I_{c}\right]\left[N_{c}\right]\right)|J(\xi)| d \xi$ is the viscoelastic core's mass matrix and $|J(\xi)|$ is the Jacobian.

Next, substituting $\mathbf{u}_{c}$ from Eq. 4.9 into Eq. 3.48, the variational strain energy yields:

$$
\delta \bar{U}_{c}=b \int_{-1}^{1}\left(\delta q_{e}^{T}\left[B_{c}\right]^{T}\left[C_{c}\right]\left[B_{c}\right] q_{e}\right)|J(\xi)| d \xi=\delta q_{e}^{T}\left[K_{c}\right]^{e} q_{e}
$$

where the viscoelastic layer's stiffness matrix is $\left[K_{c}\right]^{e}=b \int_{-1}^{1}\left(\left[B_{c}\right]^{T}\left[C_{c}\right]\left[B_{c}\right]\right)|J(\xi)| d \xi$, and $\left[B_{c}\right]=\left[D_{c}\right]\left[N_{c}\right]$

The variation of the anelastic strain energy is similarly established by replacing $\mathbf{u}_{c}$ in Eq. 3.49 with Eq. 4.9, yielding:

$$
\delta \widetilde{\bar{U}}_{c}=b \int_{-1}^{1}\left(\delta q_{e}^{T}\left[B_{c}\right]^{T}\left[\widetilde{C}_{c}\right]\left[B_{c}\right] q_{e}\right)|J(\xi)| d \xi=\delta q_{e}^{T}\left[\widetilde{K}_{c}\right]^{e} q_{e}
$$

where the anelastic portion of the viscoelastic core's stiffness matrix is $\left[\widetilde{K}_{c}\right]^{e}=b \int_{-1}^{1}\left(\left[B_{c}\right]^{T}\left[\widetilde{C}_{c}\right]\left[B_{c}\right]\right)|J(\xi)| d \xi$. Recall that the anelastic stiffness is a factor of the 
elastic stiffness, $\left[\widetilde{K}_{c}\right]^{e}=\eta \frac{E_{\infty}-E_{0}}{E_{0}}\left[K_{c}\right]^{e}$ from Eq. 3.49 .

Finally, taking $\widetilde{\mathbf{u}}_{c}$ from Eq. 4.10 and substituting into Eq. 3.50 gives the final form of the variation of work $\delta \widetilde{W}_{c}$ as:

$$
\begin{aligned}
\delta \widetilde{W}_{c} & =\eta \frac{E_{\infty}}{E_{0}} \sum_{j=1}^{N_{t}}\left(\prod_{p=1}^{j} \frac{p-\alpha-1}{p}\right) \times b \int_{-1}^{1}\left(\left\{\delta q_{e}^{T}\right\}\left[B_{c}\right]^{T}\left[C_{c}\right]\left[B_{c}\right]\left\{\widetilde{q}_{e}(t-j \Delta t)\right\}\right)|J(\xi)| d \xi \\
& =\delta q_{e}^{T} \widetilde{F}_{c}^{e}
\end{aligned}
$$

where the excited force in the viscoelastic layer is:

$$
\widetilde{F}_{c}^{e}=\eta \frac{E_{\infty}}{E_{0}}\left[K_{c}\right]^{e} \sum_{j=1}^{N_{t}}\left(\prod_{p=1}^{j} \frac{p-\alpha-1}{p}\right) \times \widetilde{q}_{e}(t-j \Delta t)
$$

\subsection{PRFC Laminate (Top Layer)}

The PFRC laminate displacement vector is $\mathbf{u}_{t}^{T}=\left\{u_{t} w_{t} \phi_{t}\right\}$. It can be further expanded to:

$$
\mathbf{u}_{t}=\left[N_{t}\right] q_{e}
$$

where

$$
\left[N_{t}\right]=\left[\begin{array}{ccccccccccccccccccccc}
0 & 0 & 0 & 0 & 0 & 0 & N_{1 \xi} & 0 & 0 & 0 & 0 & 0 & 0 & 0 & 0 & 0 & 0 & 0 & N_{2 \xi} & 0 & 0 \\
0 & 0 & 0 & 0 & 0 & 0 & 0 & N_{3 \xi} & 0 & 0 & 0 & N_{4 \xi} & 0 & 0 & 0 & 0 & 0 & 0 & 0 & N_{5 \xi} & 0 \\
0 & 0 & 0 & 0 & 0 & 0 & 0 & 0 & N_{1 \xi} & 0 & 0 & 0 & 0 & 0 & 0 & 0 & 0 & 0 & 0 & 0 & N_{2 \xi}
\end{array}\right]
$$

Subsequently, by substituting Eq. 4.16 into Eq. 3.78, the variation of the kinetic energy of the FGM beam is written as:

$$
\delta T_{t}=b \int_{-1}^{1} \rho_{t}\left(\delta q_{e}^{T}\left[N_{t}\right]^{T}\left[I_{t}\right]\left[N_{t}\right] \ddot{q}_{e}\right)|J(\xi)| d \xi=\delta q_{e}^{T}\left[M_{t}\right]^{e} \ddot{q}_{e}
$$

where $\left[M_{t}\right]^{e}=b \int_{-1}^{1} \rho_{t}\left(\left[N_{t}\right]^{T}\left[I_{t}\right]\left[N_{t}\right]\right)|J(\xi)| d \xi$ is the mass matrix of the PFRC laminate, $\left[I_{t}\right]=$ $\sum_{k=1}^{n} \int_{z_{k-1}}^{z_{k}}\left[\begin{array}{ccc}1 & 0 & -z_{t} \\ 0 & 1 & 0 \\ -z_{t} & 0 & z_{t}^{2}\end{array}\right] d z$, and the Jacobian $|J(\xi)|=\frac{L_{e}}{2}$.

Lastly, by substituting $\mathbf{u}_{t}$ from Eq. 4.16 into Eq. 3.81 and Eq. ??, produces the following 
the variational strain energy $\delta U_{p}$ and virtual work $\delta \hat{W}_{p}$ in the PFRC laminate:

$$
\begin{gathered}
\delta U_{t}=b \int_{-1}^{1}\left(\delta q_{e}^{T}\left[B_{t}\right]^{T}\left[\begin{array}{cc}
A & B \\
B & D
\end{array}\right]\left[B_{t}\right] q_{e}\right)|J(\xi)| d \xi=\delta q_{e}^{T}\left[K_{t}\right]^{e} q_{e} \\
\delta \hat{W}_{t}=-b \int_{-1}^{1} \delta q_{e}^{T}\left[B_{t}\right]^{T}\left[\begin{array}{c}
N_{x}^{t} \\
0 \\
M_{x}^{t} \\
0
\end{array}\right]|J(\xi)| d \xi=\delta q_{e}^{T} \hat{F}_{t}^{e}
\end{gathered}
$$

where the stiffness matrix is $\left[K_{t}\right]^{e}=b \int_{-1}^{1}\left(\left[B_{t}\right]^{T}\left[\begin{array}{cc}A & B \\ B & D\end{array}\right]\left[B_{t}\right]\right)|J(\xi)| d \xi$, and $\left[B_{b}\right]=\left[D_{b}\right]\left[N_{b}\right]$ and the excited force in the PRFC laminate is

$$
\hat{F}_{t}^{e}=-b \int_{-1}^{1} \delta q_{e}^{T}\left[B_{t}\right]^{T}\left[\begin{array}{c}
N_{x}^{t} \\
0 \\
M_{x}^{t} \\
0
\end{array}\right]|J(\xi)| d \xi \text { with }\left[B_{t}\right]=\left[\begin{array}{c}
D_{t}^{0} \\
D_{t}^{1}
\end{array}\right]\left[N_{t}\right] .
$$

\subsection{External Force}

The ACLD beam not only experiences work done from the viscoelastic core and PRFC laminate, but also encounters work done by an external force $F_{e}$, which is applied at a given position $\zeta_{f}$ in a certain element, formulated as:

$$
\delta W_{e}=\delta q_{e}^{T} F_{e} \int_{-1}^{1}[N]^{T} \mathbf{M}^{T} \delta\left(\zeta-\zeta_{f}\right) d \zeta
$$

where

$$
[N]=\left[\begin{array}{ccccccccccccccccccccc}
N_{1 \xi} & 0 & 0 & 0 & 0 & 0 & 0 & 0 & 0 & 0 & 0 & 0 & N_{2 \xi} & 0 & 0 & 0 & 0 & 0 & 0 & 0 & 0 \\
0 & N_{3 \xi} & 0 & 0 & 0 & 0 & 0 & 0 & 0 & N_{4 \xi} & 0 & 0 & 0 & N_{5 \xi} & 0 & 0 & 0 & 0 & 0 & 0 & 0 \\
0 & 0 & N_{1 \xi} & 0 & 0 & 0 & 0 & 0 & 0 & 0 & 0 & 0 & 0 & 0 & N_{2 \xi} & 0 & 0 & 0 & 0 & 0 & 0 \\
0 & 0 & 0 & N_{1 \xi} & 0 & 0 & 0 & 0 & 0 & 0 & 0 & 0 & 0 & 0 & 0 & N_{2 \xi} & 0 & 0 & 0 & 0 & 0 \\
0 & 0 & 0 & 0 & N_{1 \xi} & 0 & 0 & 0 & 0 & 0 & 0 & 0 & 0 & 0 & 0 & 0 & N_{2 \xi} & 0 & 0 & 0 & 0 \\
0 & 0 & 0 & 0 & 0 & N_{3 \xi} & 0 & 0 & 0 & 0 & N_{4 \xi} & 0 & 0 & 0 & 0 & 0 & 0 & N_{5 \xi} & 0 & 0 & 0 \\
0 & 0 & 0 & 0 & 0 & 0 & N_{1 \xi} & 0 & 0 & 0 & 0 & 0 & 0 & 0 & 0 & 0 & 0 & 0 & N_{2 \xi} & 0 & 0 \\
0 & 0 & 0 & 0 & 0 & 0 & 0 & N_{3 \xi} & 0 & 0 & 0 & N_{4 \xi} & 0 & 0 & 0 & 0 & 0 & 0 & 0 & N_{5 \xi} & 0 \\
0 & 0 & 0 & 0 & 0 & 0 & 0 & 0 & N_{1 \xi} & 0 & 0 & 0 & 0 & 0 & 0 & 0 & 0 & 0 & 0 & 0 & N_{2 \xi}
\end{array}\right]
$$


and $\mathbf{M}=\left[\begin{array}{lllllllllllllllllllll}0 & 0 & 0 & 0 & 0 & 0 & 0 & 0 & 0 & 0 & 0 & 0 & 0 & 0 & 0 & 0 & 0 & 0 & 0 & 1 & 0\end{array}\right]$ is a Boolean mapping vector that shows the vertical displacement for $w_{t}$ in the last node.

\subsection{Equation of Motion}

The extended Hamilton's principle from Eq 3.18 is utilized to form the element governing equation of motion at a given time $t$ as:

$$
\left(\left[M_{b}\right]^{e}+\left[M_{c}\right]^{e}+\left[M_{p}\right]^{e}\right) \ddot{q}_{e}(t)+\left(\left[K_{b}\right]^{e}+\left[K_{c}\right]^{e}+\left[\widetilde{K}_{c}\right]^{e}+\left[K_{p}\right]^{e}\right) q_{e}(t)=F_{e}(t)+\widetilde{F}_{c}^{e}(t)+\hat{F}_{t}^{e}(t)
$$

Note that the modified loading in the viscoelastic core $\widetilde{F}_{c}^{e}$ is negative on the right-handside of the above governing equation. The global system governing equations of motion are obtained by employing standard finite element method assembling techniques [39]. The global system governing equations of motion can be simplified as:

$$
[\mathbf{M}] \ddot{q}(t)+[\mathbf{K}+\widetilde{\mathbf{K}}] q(t)=\mathbf{F}(t)+\widetilde{\mathbf{F}}(t)+\hat{\mathbf{F}}(t)
$$

where $[\mathbf{M}],[\mathbf{K}+\widetilde{\mathbf{K}}], \mathbf{F}, \widetilde{\mathbf{F}}$, and $\hat{\mathbf{F}}$ symbolize the global mass matrix, stiffness matrix, external force vector, viscoelastic force vector, and electrical force vector, respectively.

\subsection{Time Integration}

The Newmark time-integration method [18, 31, 39] is employed to solve the global system governing equations of motion, Eq. 4.22, In order to incorporate the viscoelastic property of the core through fractional derivative, the classical Newmark algorithm is modified to have a new parameter for the storage of the anelastic displacement history. To ensure that the system is unconditionally stable, the Newmark parameters, $\beta=1 / 4$ and $\gamma=1 / 2$, were employed. Appendix A contains the Matlab code that was used to simulate the three-layered model. 


\section{Chapter 5}

\section{Numerical Simulation}

\subsection{Formulation Comparison with Literature}

In order to verify the current formulation, three simulations are performed and their results are compared to those in the literature. First, the work by Amarani et al. [13] on the free vibration analysis of a sandwich beam with a functionally graded (FG) core is revisited using the present formulation. The next simulation validates the viscoelastic property modeling of the ACLD beam. It requires modifying the current formulation and comparing it to the study by Galucio et al. [18] where the dynamic analysis of a sandwich beam with a viscoelastic core was investigated. Lastly, the dynamic analysis of a cantilever beam with a piezoelectric top layer from Bekuit et al. [6] is considered. Note that Quasi-2D EulerBernoulli (Q2DE) denotes the ACLD beam formulation where both the top and bottom layers are modeled as Euler-Bernoulli beams and the core is modeled via a higher-order beam theory. Similarly, quasi-2D Timoshenko (Q2DT) denotes the ACLD beam formulation where the core is modeled by a higher-order theory and the top and bottom layers as Timoshenko beams, as described in chapter 3 .

\subsubsection{Sandwich Beam with FG core}

The current formulation is modified to study the free vibration analysis of a sandwich beam with FG core such as that by Amirani et al. [13. The sandwich beam schematic is shown in Figure 5.1, wherein the beam consists of a FG core, composed of aluminum (metal) and zirconia (ceramic), and face sheets that are made of steel alloy. Two boundary conditions are analyzed, namely, fully- and partially- clamped cantilever. The fully-clamped boundary condition is the case when all three layers are fixed at one end. The partially-clamped case is when only the face sheets are fixed. Their properties are tabulated in Table 5.1. The 
geometrical properties of the beam are length $\mathrm{L}=1000 \mathrm{~mm}$, face sheet thickness $h_{f}=3$ $\mathrm{mm}$, and core thickness $h_{c}=14 \mathrm{~mm}$.

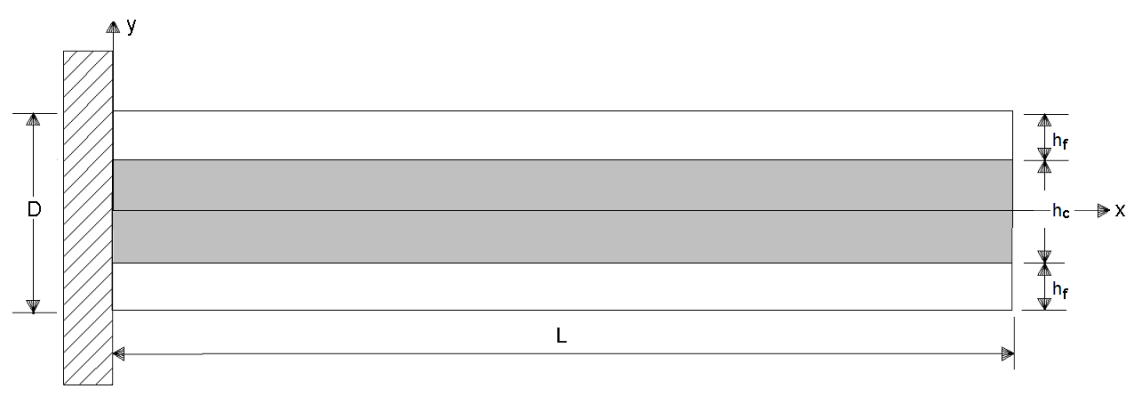

Figure 5.1: Schematic of a fully-clamped sandwich beam with FG core.

Table 5.1: Material properties of sandwich beam with FG core.

\begin{tabular}{ccccc}
\hline \hline \multirow{2}{*}{ Property } & Face sheets & & \multicolumn{2}{c}{ FG Core } \\
\cline { 2 - 3 } \cline { 5 - 5 } & Steel & & Aluminum & Zirconia \\
\hline E $(\mathrm{GPa})$ & 210 & & 70 & 151 \\
$\nu$ & 0.3 & & 0.3 & 0.3 \\
$\rho\left(\mathrm{kg} / \mathrm{m}^{3}\right)$ & 7860 & & 2700 & 5700 \\
\hline
\end{tabular}

Following Amirani et al. [13] rule of mixtures technique, also known as power-law functionally graded material (P-FGM) in Ref. [17], the first 10 natural frequencies are found using the present quasi-2D formulation. The natural frequencies of the sandwich beam with a P-FGM core for fully- and partially- clamped cantilevered boundary conditions are reproduced in Table 5.2. A total of 100 elements along the length of the beam are used to produce the results for the quasi-2D formulations. 
Table 5.2: First 10 natural frequencies of sandwich beam with a P-FGM $(\mathrm{p}=1)$ core.

\begin{tabular}{|c|c|c|c|c|c|c|c|}
\hline \multirow[b]{3}{*}{ Mode } & \multicolumn{7}{|c|}{ Frequency $(\mathrm{Hz})$} \\
\hline & \multirow[b]{2}{*}{ Classical } & \multicolumn{3}{|c|}{ fully-clamped cantilevered } & \multicolumn{3}{|c|}{ partially-clamped cantilevered } \\
\hline & & Ref. 13 & Q2DE & Q2DT & Ref.13] & Q2DE & Q2DT \\
\hline 1 & 18.53 & 18.32 & 18.73 & 18.73 & 18.31 & 18.71 & 18.71 \\
\hline 2 & 115.83 & 114.66 & 117.10 & 117.07 & 114.65 & 116.96 & 116.95 \\
\hline 3 & 322.87 & 320.57 & 326.54 & 326.43 & 320.57 & 326.21 & 326.12 \\
\hline 4 & 628.62 & 626.81 & 636.17 & 635.83 & 626.80 & 635.59 & 635.29 \\
\hline 5 & 1030.60 & 1033.70 & 1043.84 & 1043.04 & 1033.50 & 1043.00 & 1042.23 \\
\hline 6 & 1285.29 & 1290.10 & 1320.60 & 1320.42 & 1290.10 & 1315.17 & 1314.83 \\
\hline 7 & 1524.33 & 1539.60 & 1545.43 & 1543.79 & 1539.00 & 1544.33 & 1542.70 \\
\hline 8 & 2104.77 & 2143.70 & 2136.27 & 2133.29 & 2142.10 & 2134.92 & 2131.90 \\
\hline 9 & 2766.44 & 2844.00 & 2811.29 & 2806.29 & 2840.50 & 2809.70 & 2804.58 \\
\hline 10 & 3503.64 & 3640.00 & 3565.12 & 3557.33 & 3633.40 & 3563.29 & 3555.24 \\
\hline
\end{tabular}

Amirani et al. [13] used the element free Galerkin (EFG) method in their formulation and reported frequencies that are slightly higher than those of the classical method from modes 5 to 10. A slight difference is observed between the fully- and partially- clamped cases for both Q2DE and Q2DT, wherein the partially-clamped case continuously has smaller frequencies as expected. Generally, the Q2DT reports smaller frequencies than the Q2DE. Again this is intuitive, since the Timoshenko beam theory is more flexible than the Euler-Bernoulli beam theory. A comparison between Ref. [13] and the quasi-2D frequencies shows that the latter are smaller for the last few modes.

Further, to compare the effects of the different distribution functions, the core is altered to be exponentially functionally graded material (E-FGM) following the formulation of Ref. [17]. The material properties remained the same for zirconia and aluminum, as previously shown in Table 5.1. In order to properly compare the two distributions, the P-FGM and E-FGM method should yeild equivalent average material properties, where $G_{\text {avg }}=\frac{1}{2 h} \int_{-h}^{h} G(z) d z$. The average values were $E_{\text {avg }} \approx 105 G P a, \nu_{\text {avg }} \approx 0.3, \rho_{\text {avg }} \approx 4015$ $\mathrm{kg} / \mathrm{m}^{3}$. For P-FGM to obtain these values, the constant $\mathrm{p}$ in the formulation was set to 0.78. The first 10 natural frequencies of the sandwich beam with a P-FGM $(\mathrm{p}=0.78)$ and E-FGM core for fully-clamped and partially-clamped cantilevered boundary conditions are tabulated in Table 5.3. The natural frequencies for both P-FGM and E-FGM distributions have insignificant differences for both the fully- and partially- clamped cantilevered cases. 
Table 5.3: Fully-clamped cantilevered natural frequencies of sandwich beam with a P-FGM $(\mathrm{p}=0.78)$ and E-FGM core for $E_{\text {avg }} \approx 105 \mathrm{GPa}, \nu_{\text {avg }} \approx 0.3, \rho_{\text {avg }} \approx 4015 \mathrm{~kg} / \mathrm{m}^{3}$.

\begin{tabular}{|c|c|c|c|c|c|c|c|c|c|}
\hline \multirow[b]{3}{*}{ Mode } & \multicolumn{9}{|c|}{ Frequency $(\mathrm{Hz})$} \\
\hline & \multicolumn{3}{|c|}{ Classical } & \multicolumn{3}{|c|}{ Q2DE } & \multicolumn{3}{|c|}{ Q2DT } \\
\hline & Homogeneous & P-FGM & E-FGM & Homogeneous & P-FGM & E-FGM & Homogeneous & P-FGM & E-FGM \\
\hline 1 & 18.71 & 18.70 & 18.71 & 18.91 & 18.90 & 18.92 & 18.91 & 18.90 & 18.90 \\
\hline 2 & 116.93 & 116.88 & 116.90 & 118.20 & 118.13 & 118.15 & 118.19 & 118.10 & 118.12 \\
\hline 3 & 325.89 & 325.75 & 325.80 & 329.57 & 329.37 & 329.42 & 329.54 & 329.26 & 329.30 \\
\hline 4 & 634.32 & 634.06 & 634.15 & 641.94 & 641.55 & 641.61 & 641.84 & 641.21 & 641.27 \\
\hline 5 & 1039.62 & 1039.24 & 1039.37 & 1053.06 & 1052.41 & 1052.46 & 1052.82 & 1051.60 & 1051.65 \\
\hline 6 & 1283.28 & 1284.84 & 1284.48 & 1317.65 & 1319.52 & 1319.02 & 1317.58 & 1319.35 & 1318.86 \\
\hline 7 & 1537.08 & 1536.60 & 1536.77 & 1558.62 & 1557.65 & 1557.64 & 1558.14 & 1555.98 & 1555.99 \\
\hline 8 & 2121.45 & 2120.92 & 2121.12 & 2153.77 & 2152.44 & 2152.31 & 2152.91 & 2149.41 & 2149.30 \\
\hline 9 & 2787.05 & 2786.53 & 2786.74 & 2833.22 & 2831.51 & 2831.17 & 2831.80 & 2826.45 & 2826.14 \\
\hline 10 & 3527.96 & 3527.55 & 3527.75 & 3591.41 & 3589.34 & 3588.68 & 3589.23 & 3581.44 & 3580.82 \\
\hline
\end{tabular}

Table 5.4: Partially-clamped cantilevered natural frequencies of sandwich beam with a $\mathrm{P}$ FGM $(\mathrm{p}=0.78)$ and E-FGM core for $E_{\text {avg }} \approx 105 \mathrm{GPa}, \nu_{\text {avg }} \approx 0.3, \rho_{\text {avg }} \approx 4015 \mathrm{~kg} / \mathrm{m}^{3}$.

\begin{tabular}{|c|c|c|c|c|c|c|}
\hline \multirow[b]{3}{*}{ Mode } & \multicolumn{6}{|c|}{ Frequency $(\mathrm{Hz})$} \\
\hline & \multicolumn{3}{|c|}{ Q2DE } & \multicolumn{3}{|c|}{ Q2DT } \\
\hline & Homogeneous & P-FGM & E-FGM & Homogeneous & P-FGM & E-FGM \\
\hline 1 & 18.89 & 18.88 & 18.90 & 18.89 & 18.88 & 18.88 \\
\hline 2 & 118.08 & 118.00 & 118.02 & 118.07 & 117.98 & 118.00 \\
\hline 3 & 329.23 & 329.05 & 329.09 & 329.24 & 328.97 & 329.00 \\
\hline 4 & 641.64 & 641.00 & 641.06 & 641.33 & 640.70 & 640.76 \\
\hline 5 & 1052.88 & 1051.61 & 1051.67 & 1052.07 & 1050.83 & 1050.89 \\
\hline 6 & 1312.69 & 1314.36 & 1313.90 & 1312.33 & 1314.03 & 1313.57 \\
\hline 7 & 1558.87 & 1556.61 & 1556.61 & 1557.14 & 1554.96 & 1554.97 \\
\hline 8 & 2154.88 & 2151.18 & 2151.06 & 2151.66 & 2148.10 & 2148.00 \\
\hline 9 & 2835.75 & 2830.03 & 2829.70 & 2830.30 & 2824.84 & 2824.54 \\
\hline 10 & 3596.03 & 3587.64 & 3586.99 & 3587.44 & 3579.49 & 3578.88 \\
\hline
\end{tabular}

For the fully-clamped cantilevered condition in Table 5.3, there is very little difference between the natural frequencies of the homogeneous, P-FGM and E-FGM core distributions.

The natural frequencies for the partially-clamped cantilevered case as tabulated in Table 5.4 and they are generally identical to those of the homogeneous, P-FGM and E-FGM core. For the last mode, however, there are greater differences in the homogeneous and FGM 
frequencies compared to the fully-clamped case. The difference in values show that the quasi-2D formulation is better able to capture the effect of the unconstrained core.

\subsubsection{Visco-Aluminum Sandwich Beam}

The example by Galucio et al. [18] is used to demonstrate the effectiveness of the present quasi-2D formulation for a sandwich beam with a viscoelastic core. The viscoelastic core is sandwiched between two aluminum layers. The beam is clamped at one end and a triangular impulse transverse load is applied at the free end, as depicted in Figure 5.2. The geometric parameters of the beam are: length $\mathrm{L}=200 \mathrm{~mm}$, width $\mathrm{b}=10 \mathrm{~mm}$, thickness of top and bottom face sheets $h_{f}=1 \mathrm{~mm}$ and thickness of core $h_{c}=0.2 \mathrm{~mm}$. The parameters of the fractional derivative model are: $\alpha=0.7915$ and $\tau=1.4052 \times 10^{-2} \mathrm{~ms}$. The mechanical properties of the elastic aluminum faces and the viscoelastic core are tablulated in Table 5.5 . The time step and number of terms in the Grünwald series (taken from the example) are $\Delta t$ $=0.25 \mathrm{~ms}$ and $\mathrm{N}=1000$, respectively.
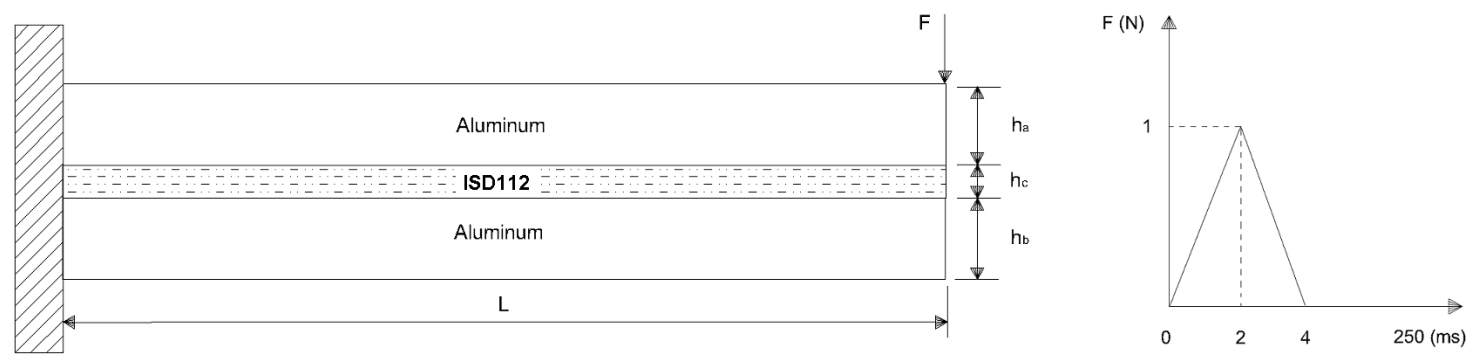

Figure 5.2: Visco-aluminum sandwich beam.

Table 5.5: Material properties of visco-aluminum sandwich beam.

\begin{tabular}{|c|c|c|c|}
\hline \multicolumn{2}{|c|}{ Face sheets } & \multicolumn{2}{|r|}{ Core } \\
\hline Property & Aluminum & Property & ISD112 (at $\left.27^{\circ} \mathrm{C}\right)$ \\
\hline $\mathrm{E}(\mathrm{GPa})$ & 70.3 & $E_{0}(\mathrm{MPa})$ & 1.5 \\
\hline & & $E_{\infty}(\mathrm{MPa})$ & 69.9495 \\
\hline$\nu$ & 0.345 & $\nu$ & 0.5 \\
\hline$\rho\left(k g / m^{3}\right)$ & 2690 & $\rho\left(\mathrm{kg} / \mathrm{m}^{3}\right)$ & 1600 \\
\hline
\end{tabular}

The transverse displacement of the beam's tip under fully-clamped cantilevered boundary condition is shown in Figure 5.3. The present quasi-2D formulation (Q2DE and Q2DT) with 
constant transverse displacement $w$ in the host (bottom) beam yields the highest peak-topeak amplitude. A trend is observed where the formulation with higher degrees of freedom (DOF) yields smaller phase shifts than that for smaller DOF. The present quasi-2D formulation and the quasi-2D with varying $w$ have smaller phase shifts than the classical response. For example, the classical method has 8 DOF, while the present quasi-2D formulation with constant $w$ has $20 \mathrm{DOF}$ and $21 \mathrm{DOF}$ for Q2DE and Q2DT, respectively. In Ref. [3], the quasi-2D with varying $w$ had 52 DOF. Note that the classical formulation is based on the classical three-layer theory from Section 1.1.1.

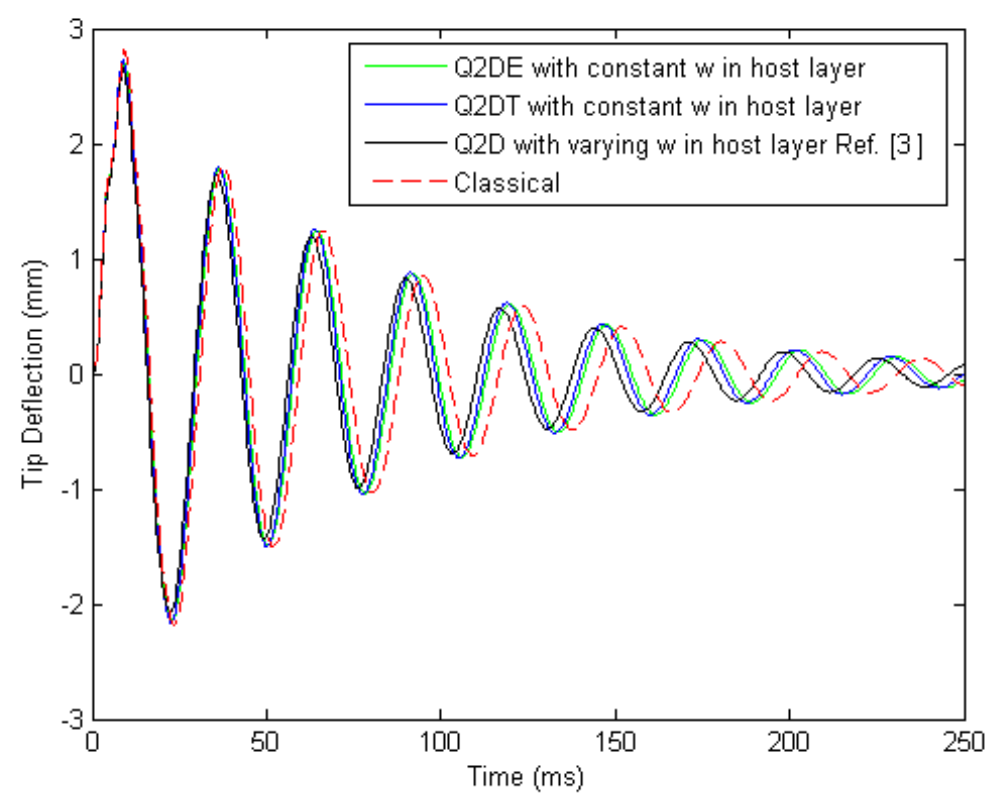

Figure 5.3: Dynamic response of visco-aluminum sandwich beam. 


\subsubsection{Visco-FGM Sandwich Beam}

Here, the viscoelastic core sandwich beam is changed from an aluminum homogeneous material to a zirconia-aluminum functionally graded (FG) material. The geometry and boundary condition of the system remain the same as the visco-aluminum sandwich beam case in Section 5.1.2. Also, the same properties for zirconia and aluminum used in Section 5.1.1 are also used in this section, as shown in Table 5.1. The top and bottom surfaces of each FG layer consist of either $100 \%$ alumuminum and/or $100 \%$ zirconia with the following approximate average properties: $E_{\text {avg }} \approx 105 \mathrm{GPa}, \nu_{\text {avg }} \approx 0.3, \rho_{\text {avg }} \approx 4015 \mathrm{~kg} / \mathrm{m}^{3}$. The first configuration consists of $100 \%$ zirconia (ceramic) material at the outer surfaces and $100 \%$ aluminum (metal) material at the inner surfaces of the top and bottom layers ((zirconia/aluminum $\left.)_{S}\right)$. Conversely, the second configuration consists of $100 \%$ aluminum (metal) material at the outer surfaces and $100 \%$ zirconia (ceramic) material at the inner surfaces of the top and bottom layers $\left((\text { aluminum/zirconia })_{S}\right)$. The schematics of these configurations are shown in Figures 5.4 and 5.5 .

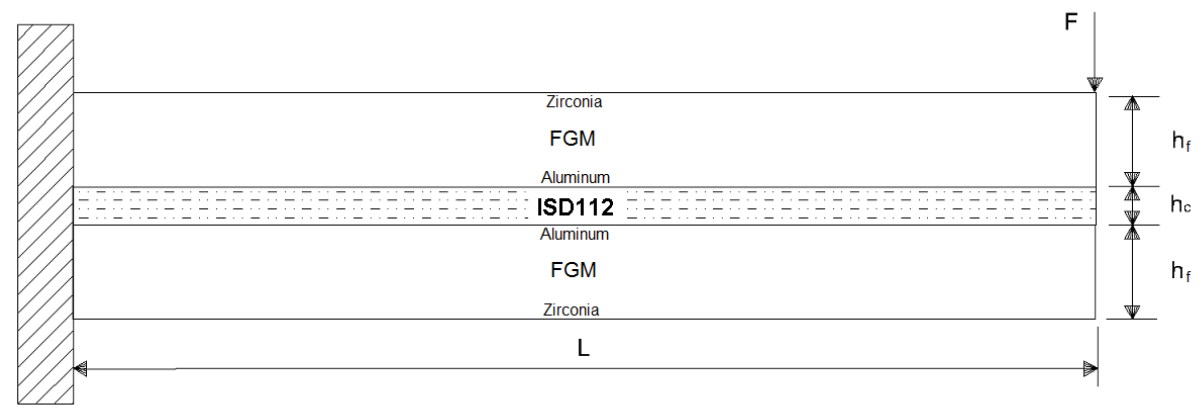

Figure 5.4: Visco-E-FGM sandwich beam with (zirconia/aluminum) $)_{S}$ orientation.

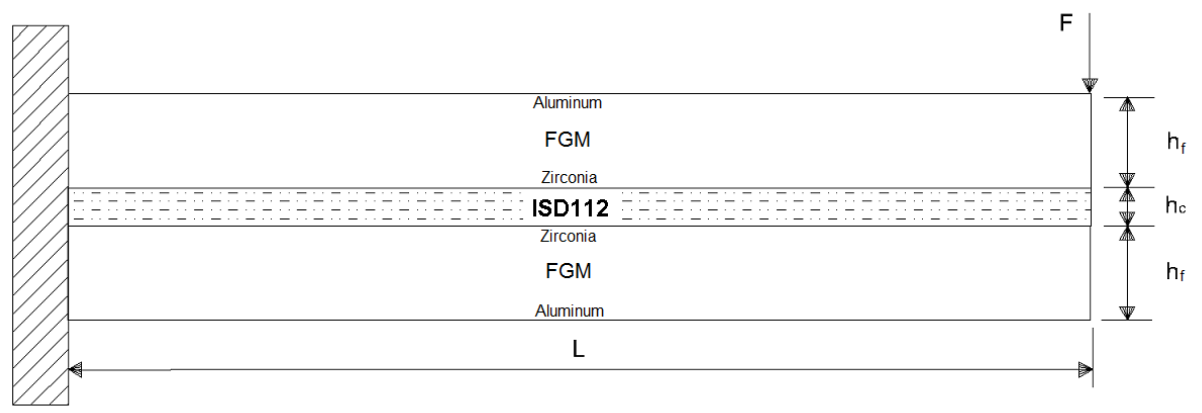

Figure 5.5: Visco-P-FGM $(\mathrm{p}=0.78)$ sandwich beam with (aluminum/zirconia $)_{S}$ orientation. 
The response of both the E-FGM and P-FGM representations for the (zirconia/aluminum) ${ }_{S}$ and (aluminum/zirconia) $)_{S}$ orientations are shown in Figures 5.6 and 5.7, and Figures 5.8 and 5.9 , respectively. With respect to the (zirconia/aluminum) ${ }_{S}$ configuration, the P-FGM representation results in smaller transverse displacements than the E-FGM representation. This is however different for the (aluminum/zirconia) $)_{S}$ orientation where the P-FGM representation produces higher transverse displacements than the E-FGM representation.

Figure 5.10 depicts a plot of all the Q2DT responses from the previous plots as well as the homogeneous representation of the top and bottom layers. The responses with the highest transverse displacement are those for the E-FGM representation for the (aluminum/zirconia) $)_{S}$ orientation and those for the P-FGM representation for the (zirconia/aluminum) $)_{S}$ orientation. Both responses result in similar transverse displacement with a slight phase shift. The smallest displacement occurs for the P-FGM with (aluminum/zirconia) S $_{\text {configuration }}$ and E-FGM with (zirconia/aluminum)s orientation. Based on these results, the use of the P-FGM distribution in a FG layer demands that the material with the highest modulus of elasticity contact the core layer in order to obtain the least amount of vibration. Conversely, using the E-FGM distribution requires that the material with the lowest elastic modulus contact the core to have the smallest amount of vibration. 


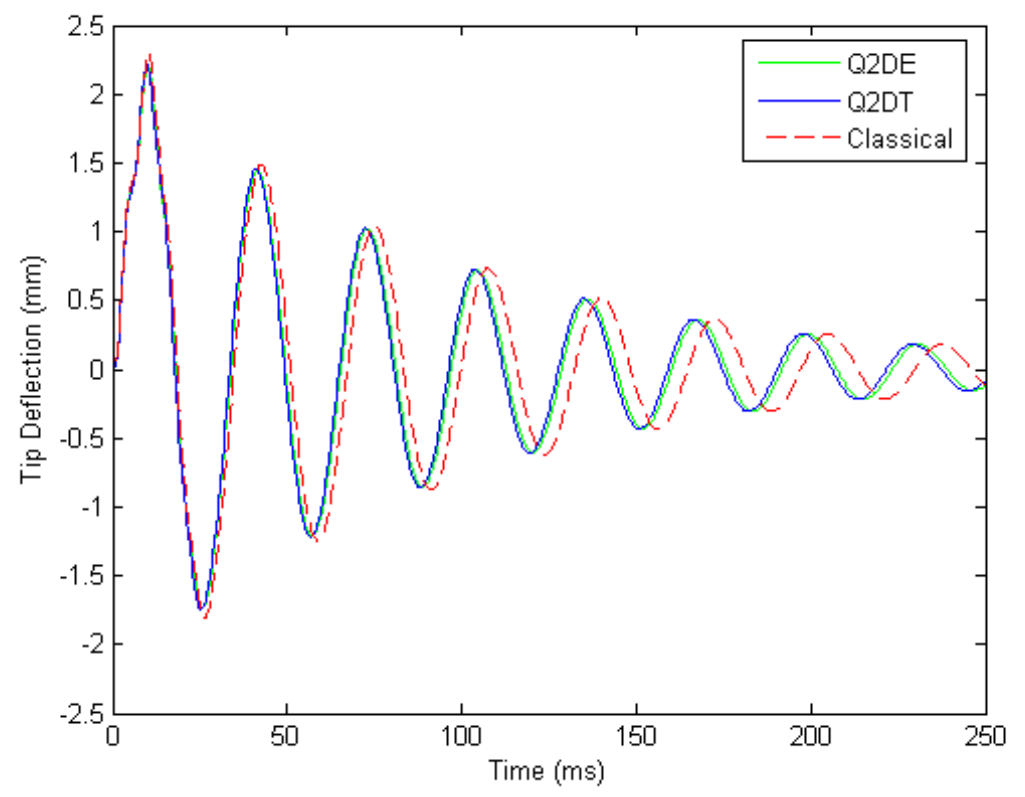

Figure 5.6: Visco-E-FGM sandwich beam with (zirconia/aluminum) $)_{S}$ orientation.

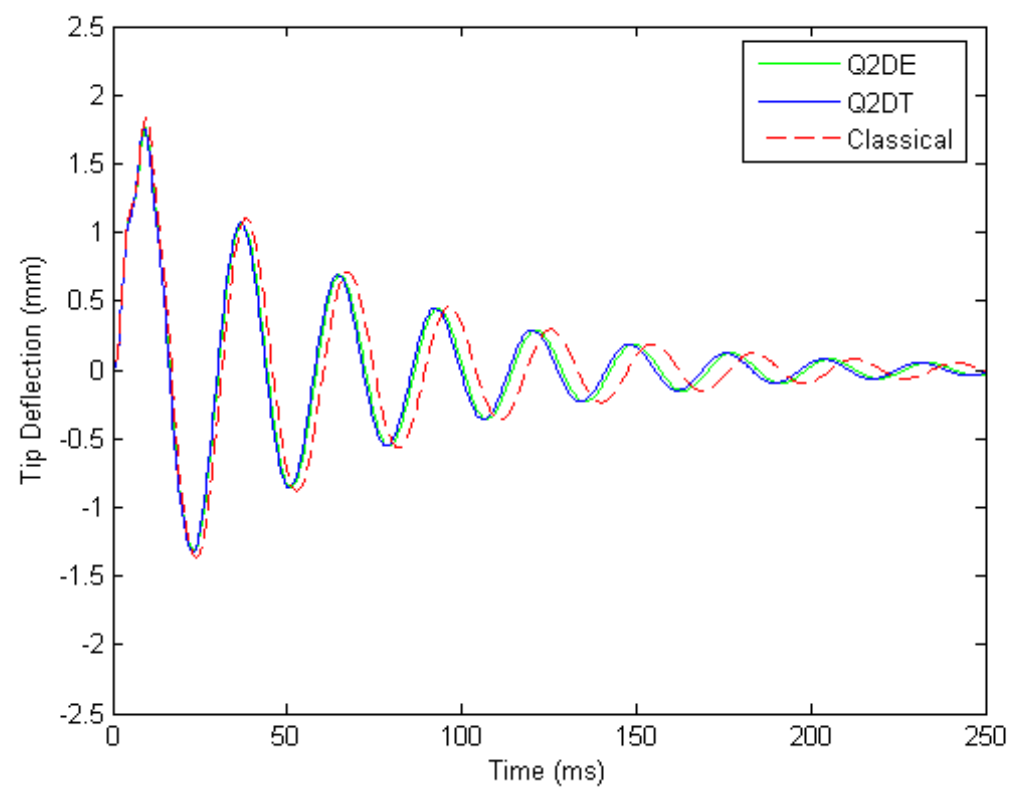

Figure 5.7: Visco-P-FGM $(\mathrm{p}=1.28)$ sandwich beam with (zirconia/aluminum $)_{S}$ orientation. 


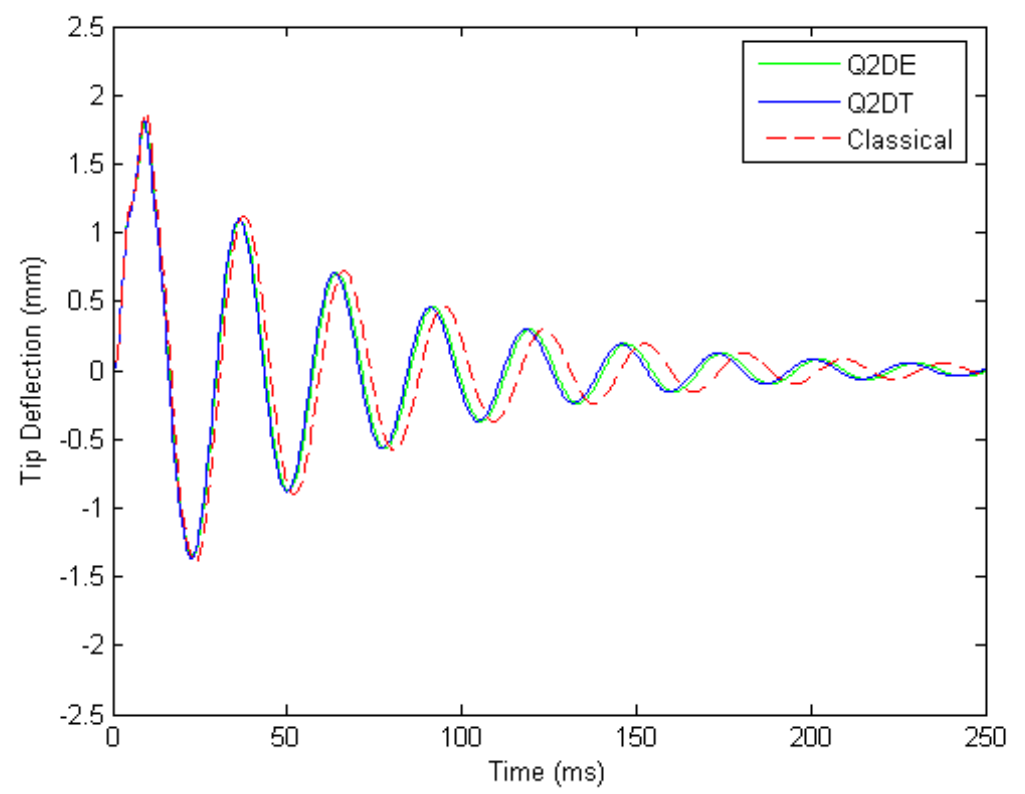

Figure 5.8: Visco-E-FGM sandwich beam with (aluminum/zirconia) $)_{S}$ orientation.

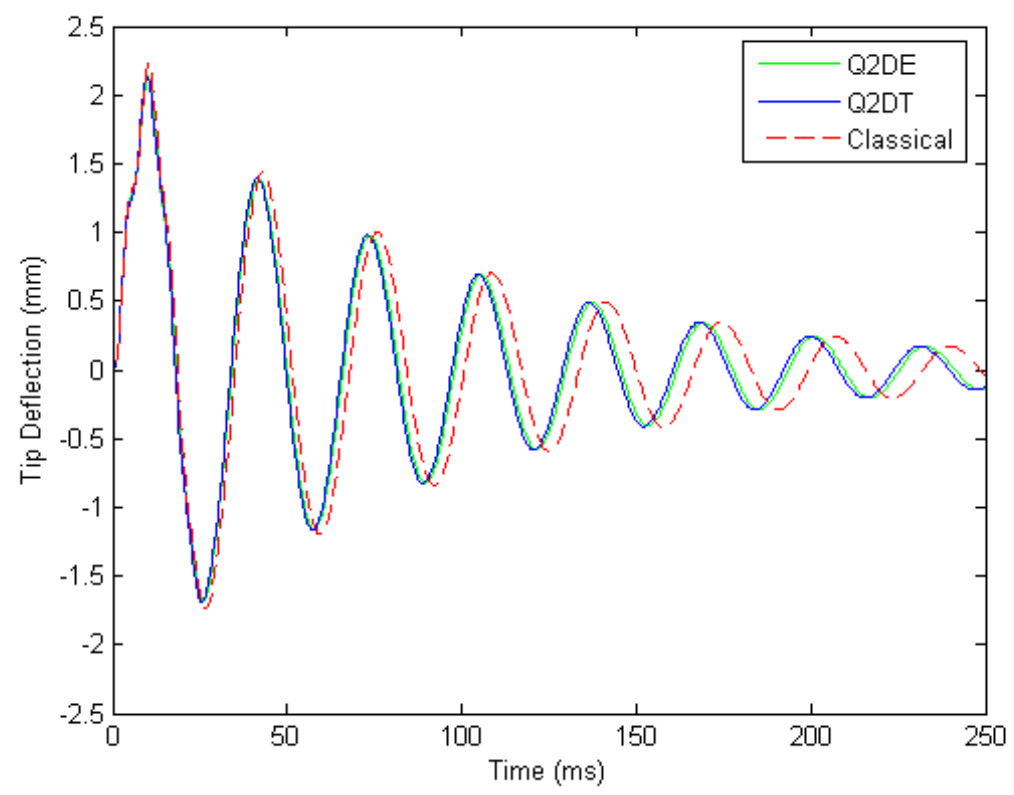

Figure 5.9: Visco-P-FGM sandwich beam with (aluminum/zirconia) $)_{s}$ orientation. 


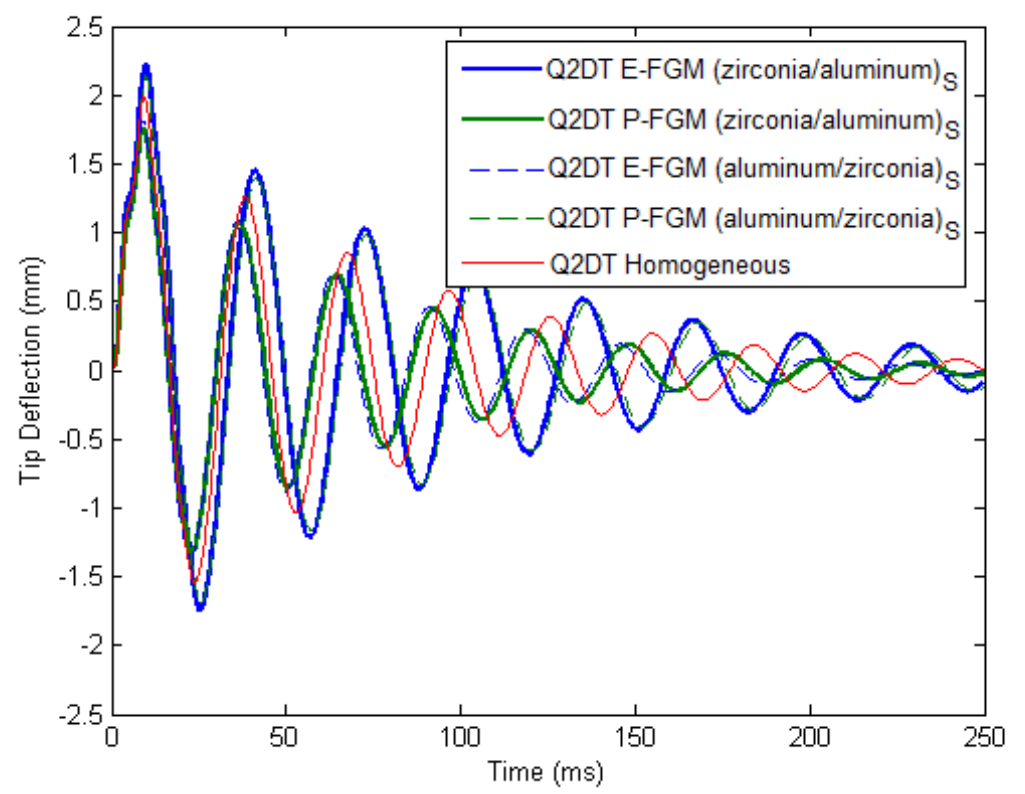

Figure 5.10: Visco sandwich beam comparison with homogeneous and FG characteristics.

\subsubsection{Cantilevered Beam with Viscoelastic Core and Piezoelectric Constraining Layer}

To further verify the accuracy of the present quasi-2D formulation, the work by Bekuit et al. [6] is reproduced and compared. The model consists of an isotropic host beam, a viscoelastic core, and a piezoelectric constraining layer. Figure 5.11 depicts the beam schematic and the triangular impulse load that is applied at the free end.
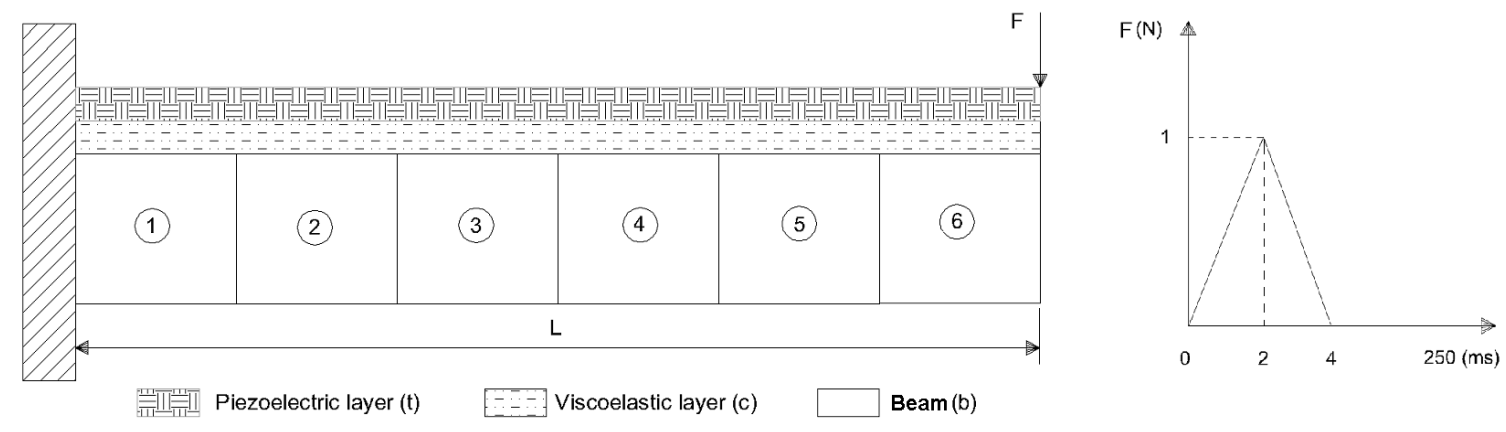

Figure 5.11: Cantilevered beam with viscoelastic core and piezoelectric constraining layer.

The geometric characteristics of the beam are: length $\mathrm{L}=300 \mathrm{~mm}$, width $\mathrm{b}=15$ 
mm, thickness of host layer $h_{b}=3 \mathrm{~mm}$, thickness of piezoelectric layer $h_{t}=1 \mathrm{~mm}$, and thickness of core $h_{c}=0.2 \mathrm{~mm}$ and $h_{c}=1.0 \mathrm{~mm}$. The number of terms in the Grünwald approximation of the fractional derivative are $\mathrm{N}=1000$ and the time step is taken as $\Delta t=$ $0.001 \mathrm{~s}$. The mechanical and electrical properties of the active constraining layer $(\mathrm{PZT} 5 \mathrm{H})$, and the mechanical properties of the host beam (steel) and the viscoelastic core (ISD112) are tabulated in Table 5.6 .

Table 5.6: Mechanical properties and piezoelectric characteristics of the cantilever beam.

\begin{tabular}{|c|c|c|c|c|c|}
\hline \multicolumn{2}{|c|}{ Constraining Layer } & \multicolumn{2}{|c|}{ Core } & \multicolumn{2}{|c|}{ Host Beam } \\
\hline Characteristic & PZT5H & Property & ISD112 & Property & Steel \\
\hline$c_{11}, c_{13}, c_{33}, c_{66}(\mathrm{GPa})$ & $126,84.1,117,23$ & $E_{0}(\mathrm{MPa})$ & 1.5 & $E(\mathrm{GPa})$ & 200 \\
\hline$e_{31}, e_{33}\left(\mathrm{C} / m^{2}\right)$ & $-6.5,23.3$ & $E_{\infty}(\mathrm{MPa})$ & 69.9495 & $\nu$ & 0.34 \\
\hline$d_{33}(\mathrm{~F} / \mathrm{m})$ & $1.3 \times 10^{-8}$ & $\nu$ & 0.5 & $\rho\left(k g / m^{3}\right)$ & 7850 \\
\hline$\rho\left(\mathrm{kg} / \mathrm{m}^{3}\right)$ & 7500 & $\rho\left(k g / m^{3}\right)$ & 1600 & & \\
\hline
\end{tabular}

The passive damping tip displacement and the active-passive damping tip displacement and voltage are obtained for the present Q2DE and Q2DT formulations. Figure 5.12 plots the response of the Q2DE, Q2DT, Ref. [6], and classical method for a core thickness of $h_{c}$ $=0.2 \mathrm{~mm}$. The first subplot, (a), depicts the tip deflection of the passively damped beam. The dynamic responses for Q2DE and Ref. [6] are identical while the Q2DT results in a slight phase shift from the two responses. All of the responses have the same amplitudes, but the conventional has an obvious phase shift from the three quasi-2D formulations. The introduction of active damping with a gain $K_{v}=100 \mathrm{~V} /(\mathrm{m} / \mathrm{s})$ is depicted in the last two subplots, (b) and (c). As a result of this active actuation, the attenuation time of the beam has been reduced. The response amplitude of the classical method did not reduce by as much as the quasi-2D formulations. Further, Figure 5.13 depicts the response with each method with a thicker core, $h_{c}=1 \mathrm{~mm}$. The response amplitude of the classical formulation is continuously higher than those with the quasi-2D formulations. A possible explanation for this is that the quasi-2D formulations dissipate more energy since they better model the shear deformation of the core. 


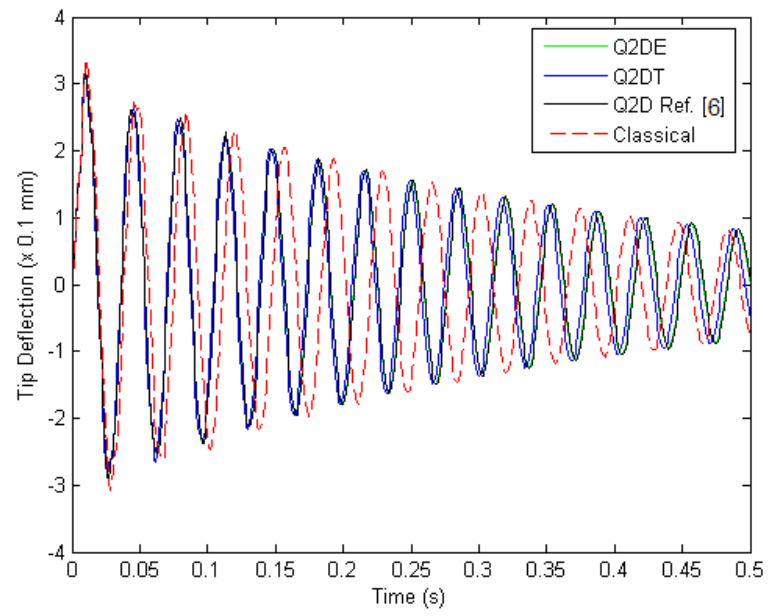

$(a)$

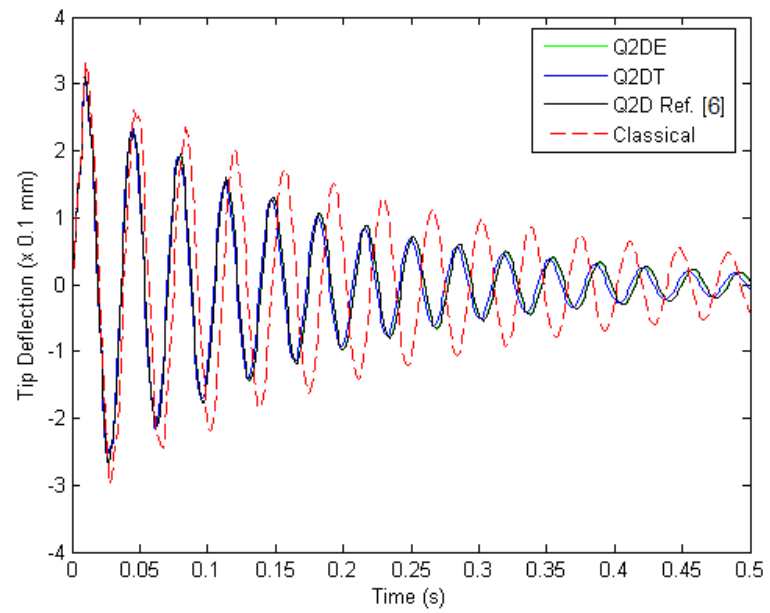

(b)

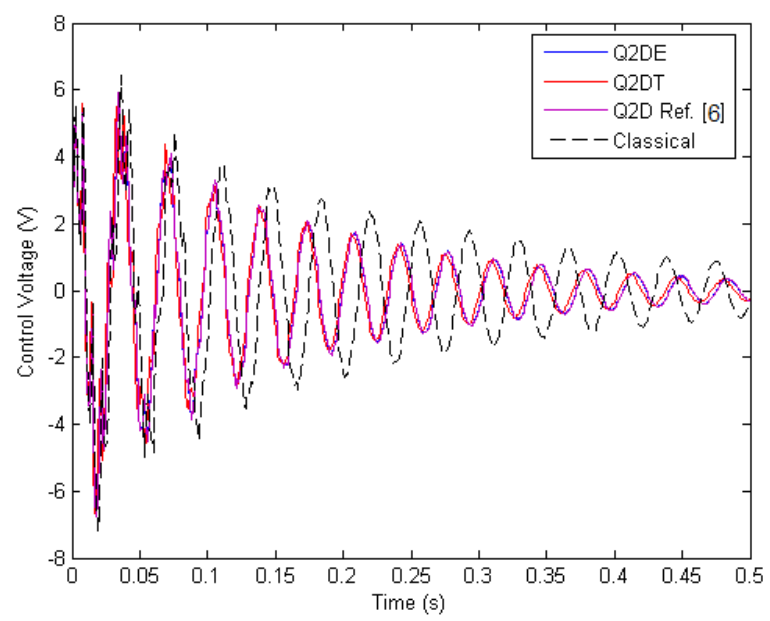

$(c)$

Figure 5.12: Tip deflections and control voltage with a PZT5H constraining layer for $h_{c}=$ $0.2 \mathrm{~mm}$. (a) Passive damping tip deflection. (b) Active-passive damping tip deflection. (c) Active-passive damping control voltage. 


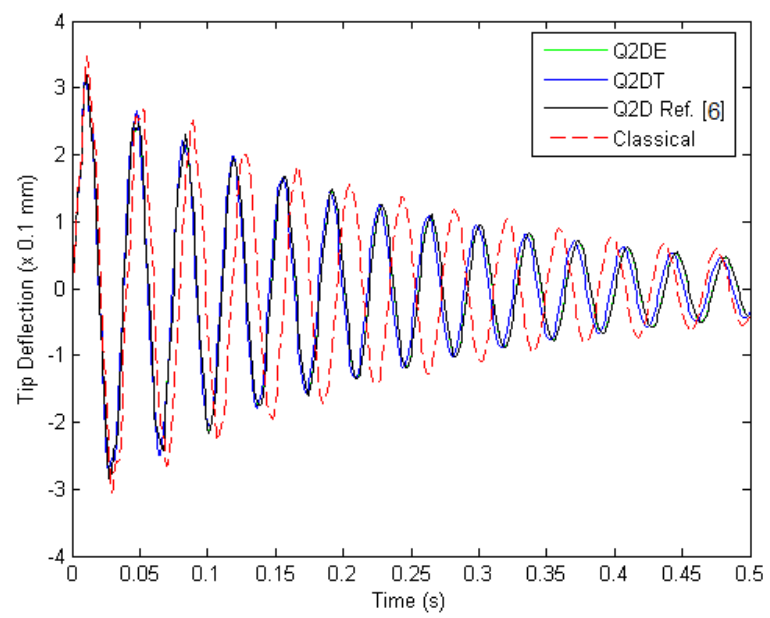

$(a)$

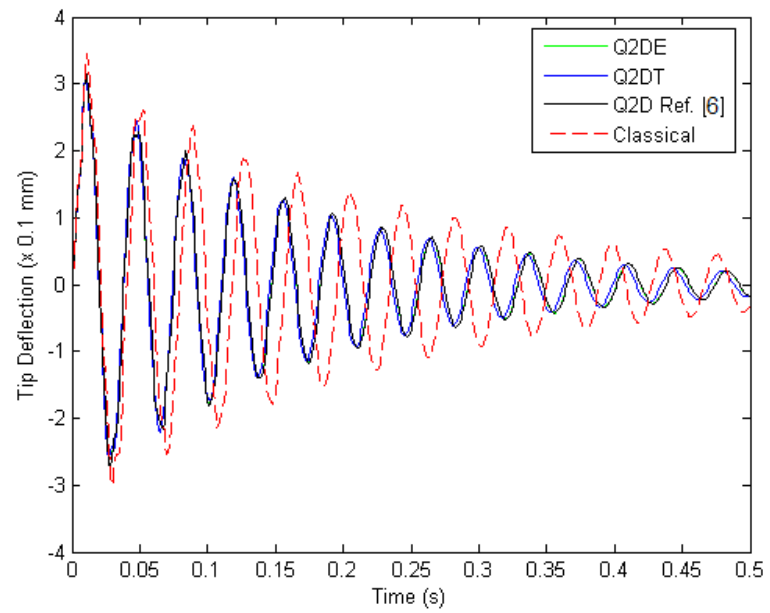

(b)

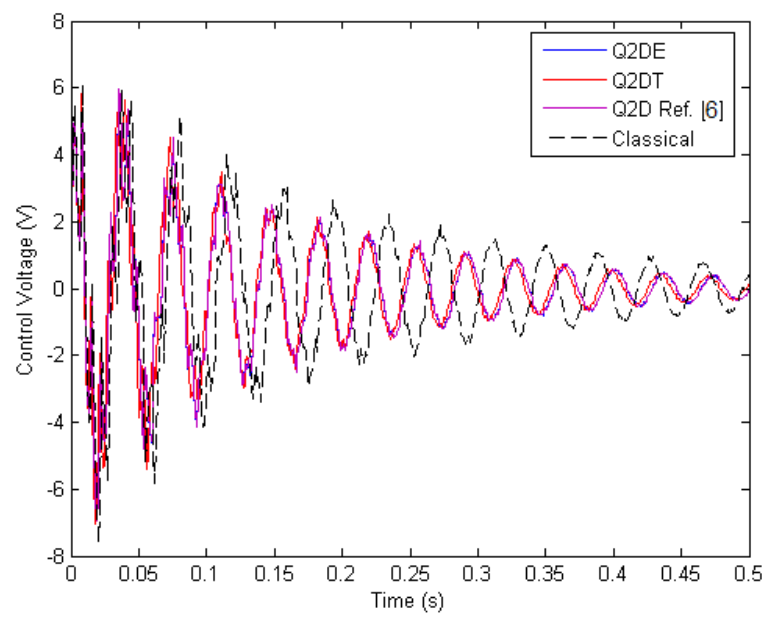

$(c)$

Figure 5.13: Tip deflections and control voltage with a PZT5H constraining layer for $h_{c}=$ $1.0 \mathrm{~mm}$. (a) Passive damping tip deflection. (b) Active-passive damping tip deflection. (c) Active-passive damping control voltage. 


\subsection{Parametric Study}

A parametric study of the current quasi-2D formulation is performed to determine the effect of laminate stacking sequence (in the PFRC laminate) and the FG material properties of the host beam. The varying parameters include the ply angle $\theta$ of the PFRC and the Young's modulus in the FG host beam. Variations in the Poisson's ratio and densities for both the metal and ceramic materials for the FG beam do not show any significant change to the response of the system. A schematic of the 3-layer system which comprises a FG host beam, viscoelastic core, and PFRC laminate constraining layer, as well as the triangular impulse load is depicted in Figure 5.14. Throughout the parametric study, the beam is consistently meshed into 6 finite elements along the span.
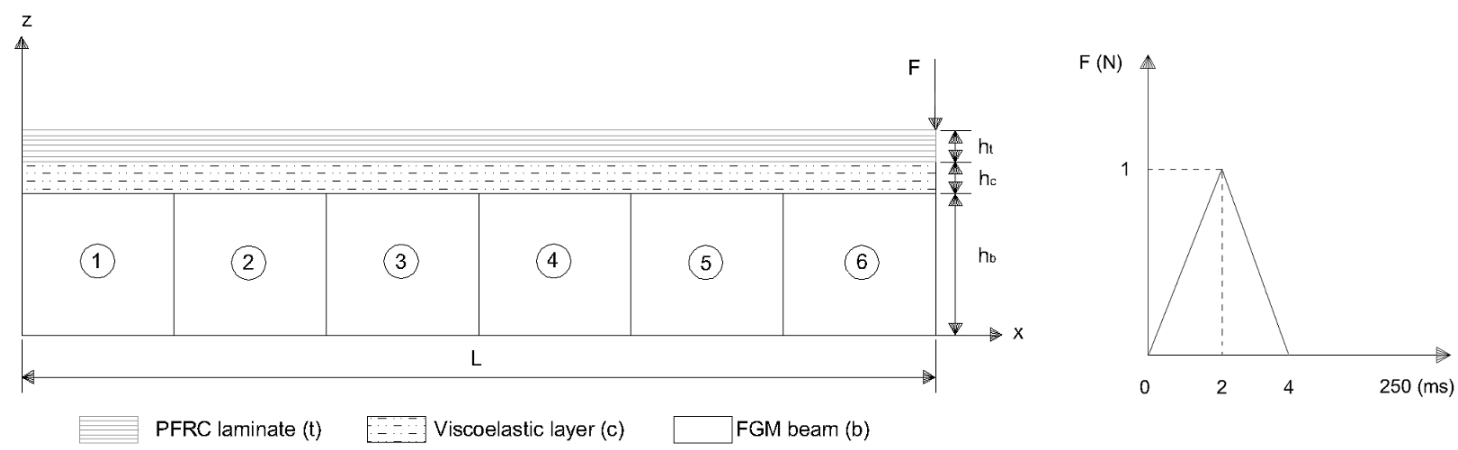

Figure 5.14: Beam with viscoelastic core and PFRC laminate constraining layer.

The geometric characteristics of the composite beam are: length $\mathrm{L}=250 \mathrm{~mm}$, width $\mathrm{b}=15 \mathrm{~mm}$, thickness of host layer $h_{b}=3 \mathrm{~mm}$, thickness of PFRC layer $h_{t}=0.75 \mathrm{~mm}$ (0.1875 mm ply x 4 plies), thickness of viscoelastic core $h_{c}=0.25 \mathrm{~mm}$. The mechanical and piezoelectric properties of the constraining layer, core, and host beam are given in Table 5.7. The effective coefficients of the PFRC used in this study were predicted utilizing the micromechanical analysis performed by Mallik and Ray [12] with a $40 \%$ volume fraction.

Table 5.7: Mechanical properties and piezoelectric characteristics of the cantilever beam.

\begin{tabular}{|c|c|c|c|c|c|}
\hline \multicolumn{2}{|c|}{ Constraining Layer } & \multicolumn{2}{|c|}{ Core } & \multicolumn{2}{|c|}{ Host Beam } \\
\hline Characteristic & PZT5H/EPOXY & Property & ISD112 & Property & Steel \\
\hline$c_{11}, c_{12}, c_{22}, c_{44}, c_{55}(\mathrm{GPa})$ & $32.6,4.3,7.2,1.05,1.29$ & $E_{0}(\mathrm{MPa})$ & 1.5 & $E(\mathrm{GPa})$ & 200 \\
\hline$e_{31}\left(C / m^{2}\right)$ & -6.76 & $E_{\infty}(\mathrm{MPa})$ & 69.9495 & $\nu$ & 0.34 \\
\hline$d_{33}(\mathrm{~F} / \mathrm{m})$ & $10.64 x 10^{-9}$ & $\nu$ & 0.5 & $\rho\left(k g / m^{3}\right)$ & 7850 \\
\hline$\rho\left(\mathrm{kg} / \mathrm{m}^{3}\right)$ & 3640 & $\rho\left(\mathrm{kg} / \mathrm{m}^{3}\right)$ & 1600 & & \\
\hline
\end{tabular}


Four types of boundary conditions are examined in each subsection: fully-clamped cantilever, partially-clamped cantilever, partially clamped-clamped, and simply-supported. The study is partitioned into two sections; the first is to study the effect of ply orientation in the PFRC laminate and the second is to examine the effect of the change in the elastic modulus of the metal and ceramic values for the FG host beam. A basic velocity feedback is implemented to control the vibration, as described in Chapter 2 .

Throughout this study, two methods of the quasi-2D are examined; Q2DE and Q2DT. As mentioned previously, quasi-2D Euler-Bernoulli (Q2DE) denotes the formulation where both the top and bottom layers are modeled as Euler-Bernoulli beams and the core is modeled using a higher-order theory. The quasi-2D Timoshenko (Q2DT), on the other hand, is the formulation where the top and bottom layers are modeled as Timoshenko beams.

\subsubsection{Effect of Ply Orientation}

To examine the effect of ply orientation on the dynamic response of the beam, the host beam remains homogeneous and isotropic with a constant elastic modulus and the number of plies in the constraining layer are fixed. A four-ply PFRC laminate with a stacking sequence of $\left[\theta_{1} / \theta_{2}\right]_{S}$ is employed. The angles $\theta_{1}$ and $\theta_{2}$ are the fiber angles relative to the $+x$ axis of each layer.

\subsubsection{Fully-clamped Cantilevered Beam Case}

The fully-clamped cantilevered beam is fully supported at the left end and free at the right as shown in Figure 5.15 .

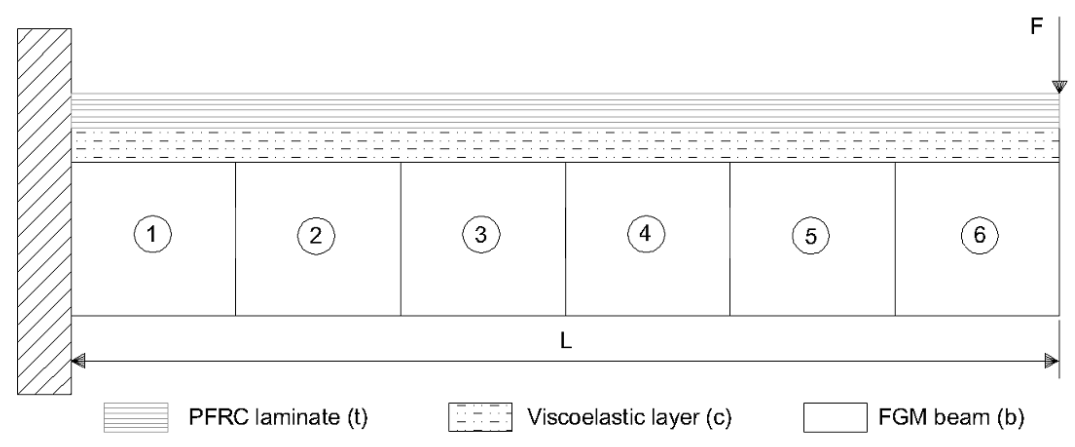

Figure 5.15: Fully-clamped cantilevered beam with ACLD treatment.

The gain that is chosen to best represent the effects of the beam vibration is $K_{v}=350$ 
$\mathrm{V} /(\mathrm{m} / \mathrm{s})$. This gain is suitable since the resulting actuation voltage is not in the breakdown voltage range, typically about 200 volts for most piezoelectric ceramics. An observation time of 0.5 seconds is selected for the beam response under impulse loading. The triangular impulse load is applied at the tip of the beam and is depicted in Figure 5.14. The time step is $\Delta t=0.001 \mathrm{~s}$ with the number of terms in the Grünwald approximation resulting in $\mathrm{N}=$ 1000.

The response of four different ply stacking sequences $\left(0_{4}^{\circ},\left[0^{\circ} / 90^{\circ}\right]_{S},\left[45^{\circ} /-45^{\circ}\right]_{S}\right.$, and $\left.\left[30^{\circ} / 60^{\circ}\right]_{S}\right)$ are determined for uncontrolled tip deflection and controlled tip deflection and actuation voltage. Figure 5.16 shows plots for uncontrolled transverse displacement of the tip node for passive damping. Figures 5.17 and 5.18 depict the response of the controlled tip deflection as well as its corresponding actuation voltage for each respective ply arrangement.

With respect to passive damping, Figure 5.16 , the $\left[45^{\circ} /-45^{\circ}\right]_{S}$ and $\left[30^{\circ} / 60^{\circ}\right]_{S}$ PFRC stacking arrangements experience the largest peak-to-peak amplitude of vibration. Since the $0_{4}^{\circ}$ arrangement is the stiffest, it experiences the smallest peak-to-peak amplitude of vibration. This is expected since the stiffer constraining layer causes more shear in the core.

With the application of active damping, the beam shows significant reduction in peakto-peak amplitude. Comparing the uncontrolled and controlled responses, Figures 5.16 and 5.17, the best controlled PFRC arrangement is the $\left[0^{\circ} / 90^{\circ}\right]_{S}$ PFRC. The control advantage is due to the contribution of $90^{\circ}$ plies that increase the flexibility of the beam, enabling the constraining layer to be more suitable for active control. This ply angle induced flexibility can also be seen for the $\left[45^{\circ} /-45^{\circ}\right]_{S}$ and $\left[30^{\circ} / 60^{\circ}\right]_{S}$ PFRC arrangements as their responses are significantly damp when actively controlled. Although the $0_{4}^{\circ}$ arrangement has the smallest peak-to-peak amplitude of passive vibration, it did not damp as much as the other three arrangements due to its stiff nature.

The actuator voltage responses in Figure 5.18 resembles the controlled deflection responses in Figure 5.17 because of their linear relationship to the response velocity. Note that the magnitudes of the voltage are well below the breakdown voltage of $200 \mathrm{~V}$. 


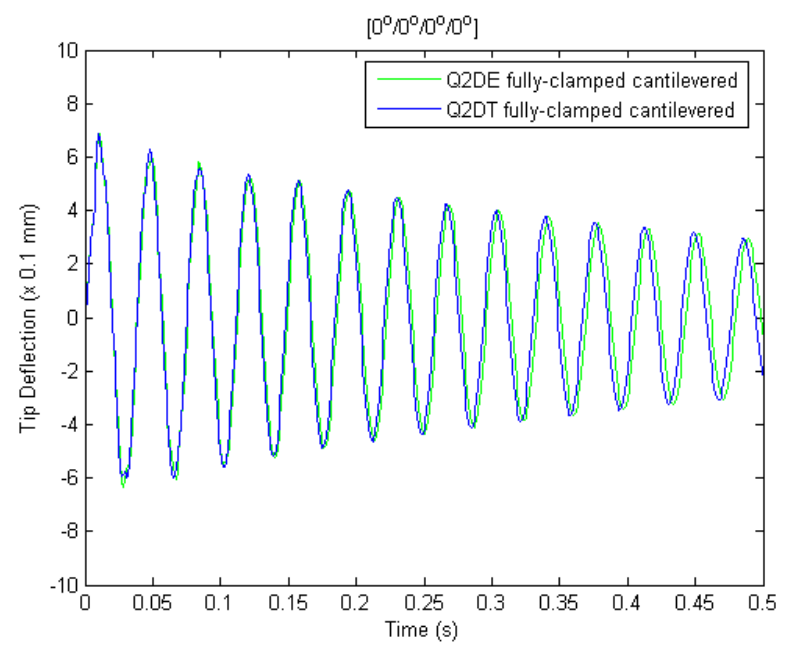

(a)

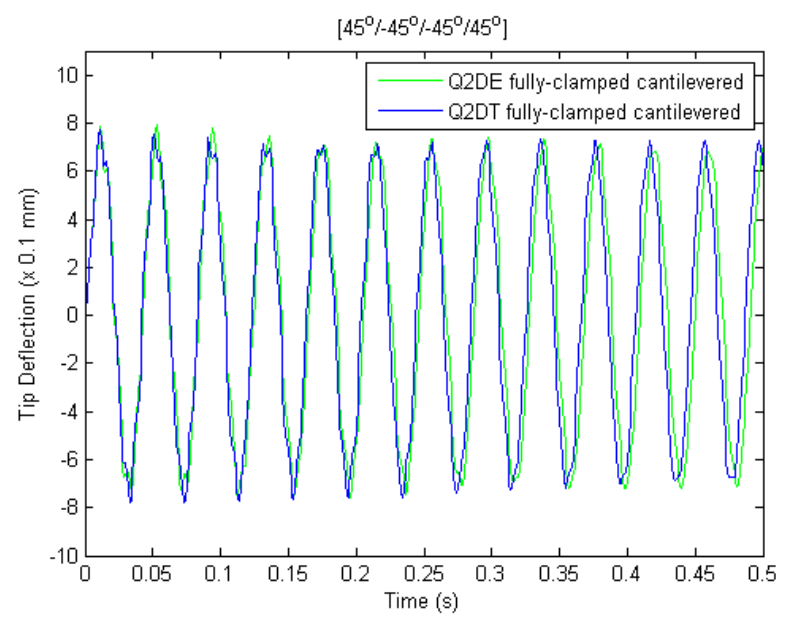

(c)

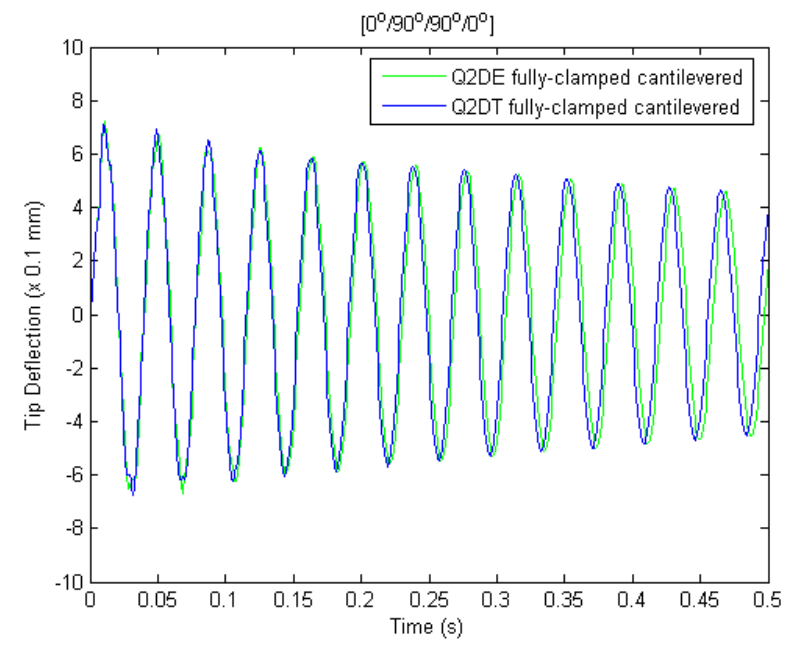

(b)

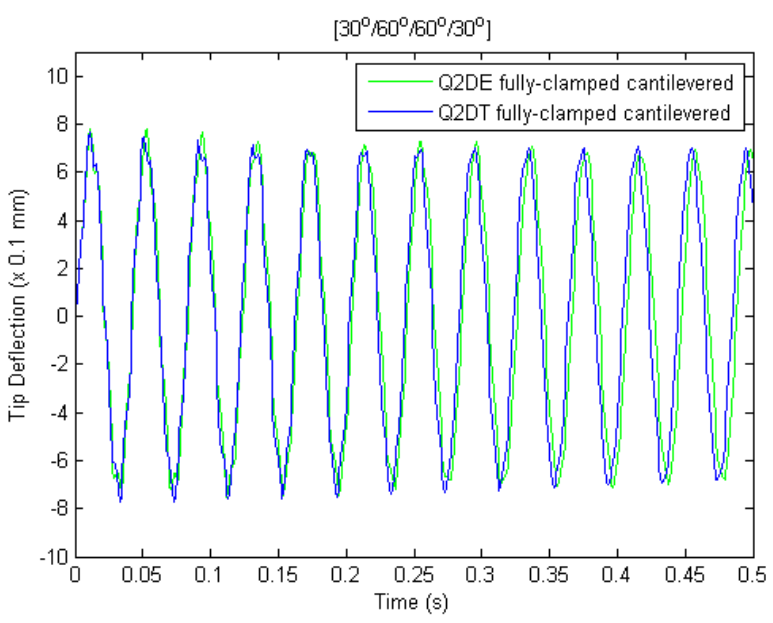

(d)

Figure 5.16: Effect of ply orientation on the tip transverse displacement for uncontrolledpassive damping of fully-clamped cantilevered beam. (a) $0_{4}^{\circ}$, (b) $\left[0^{\circ} / 90^{\circ}\right]_{S}$, (c) $\left[45^{\circ} /-45^{\circ}\right]_{S}$, (d) $\left[30^{\circ} / 60^{\circ}\right]_{S}$. 


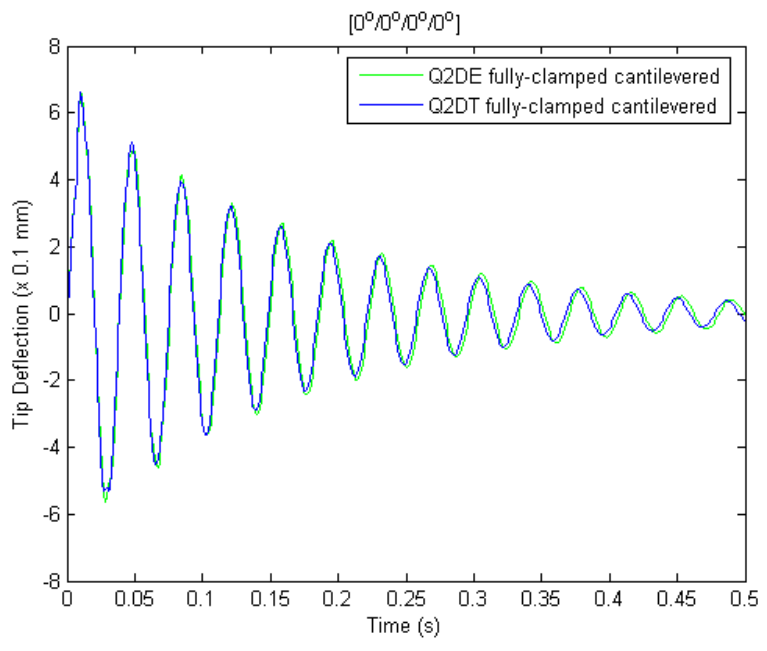

(a)

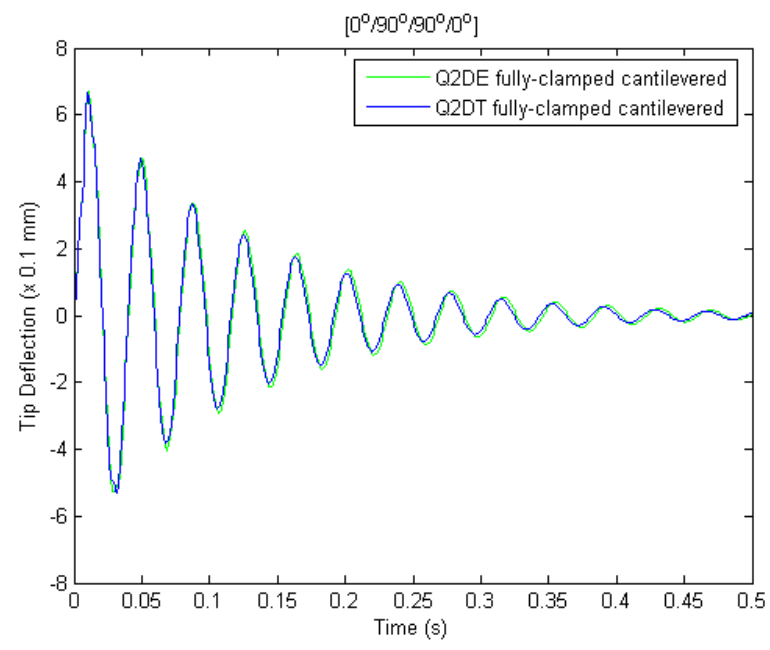

(b)

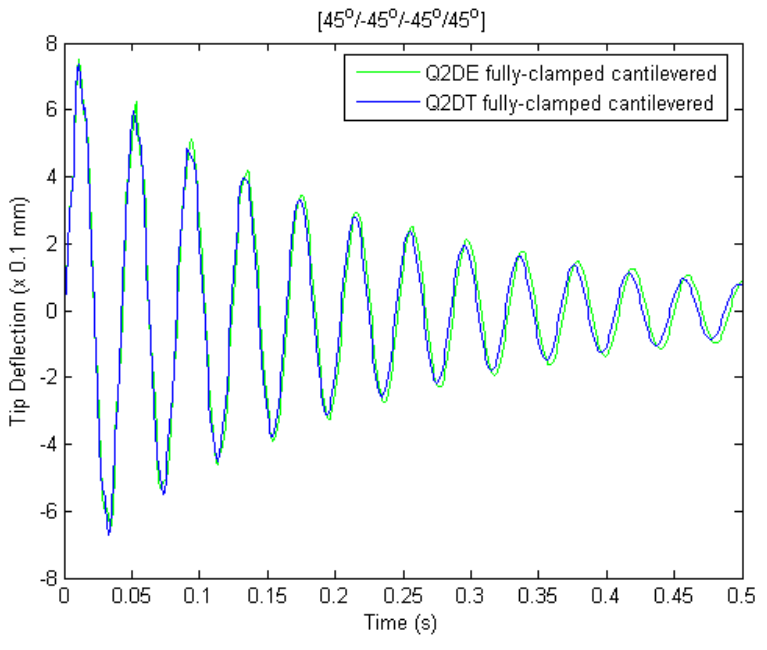

(c)

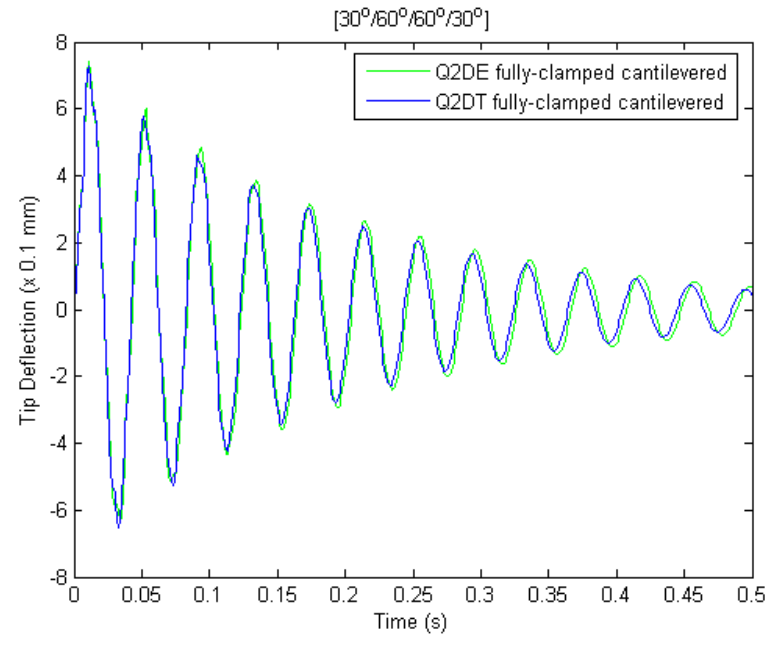

$(d)$

Figure 5.17: Effect of ply orientation on the tip transverse displacement for controlled-activepassive damping of fully-clamped cantilevered beam. (a) $0_{4}^{\circ}$, (b) $\left[0^{\circ} / 90^{\circ}\right]_{S}$, (c) $\left[45^{\circ} /-45^{\circ}\right]_{S}$, (d) $\left[30^{\circ} / 60^{\circ}\right]_{S}$. 


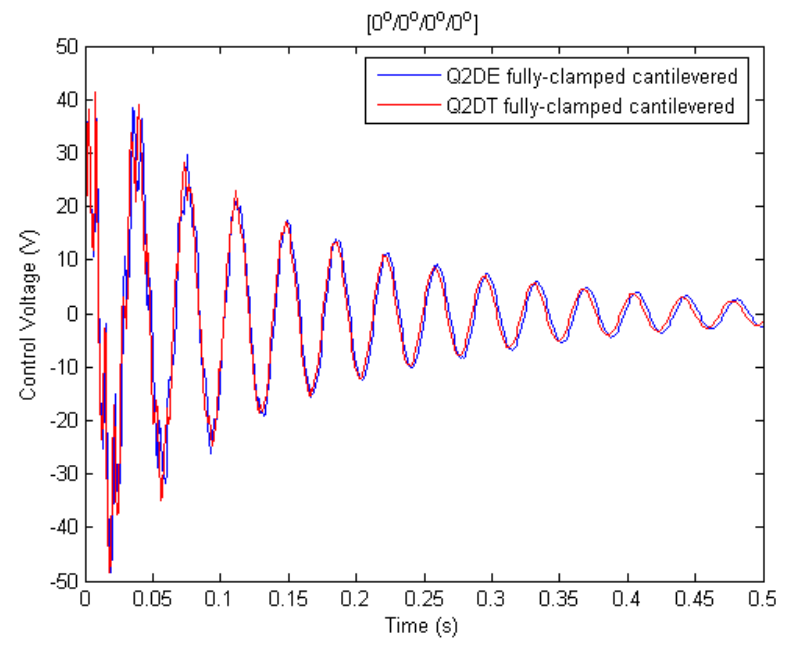

(a)

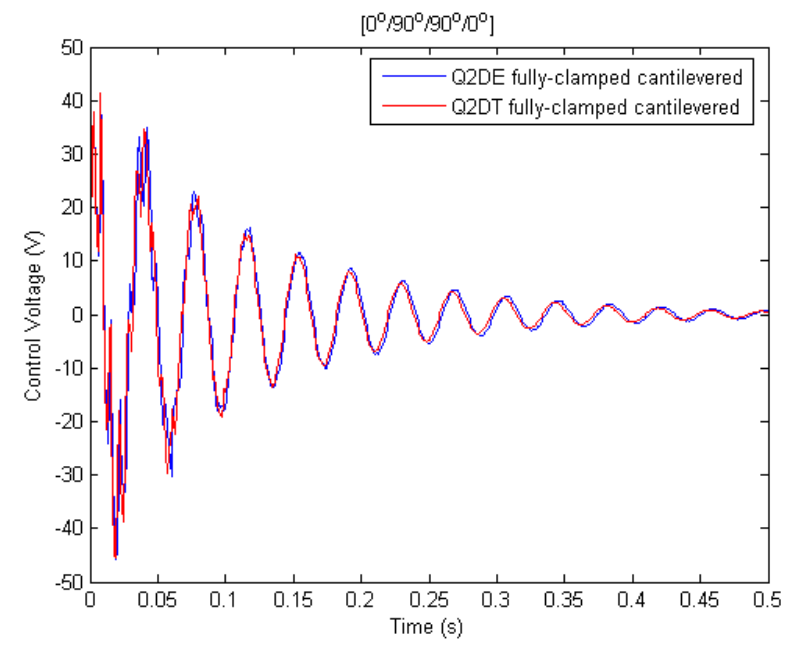

(b)

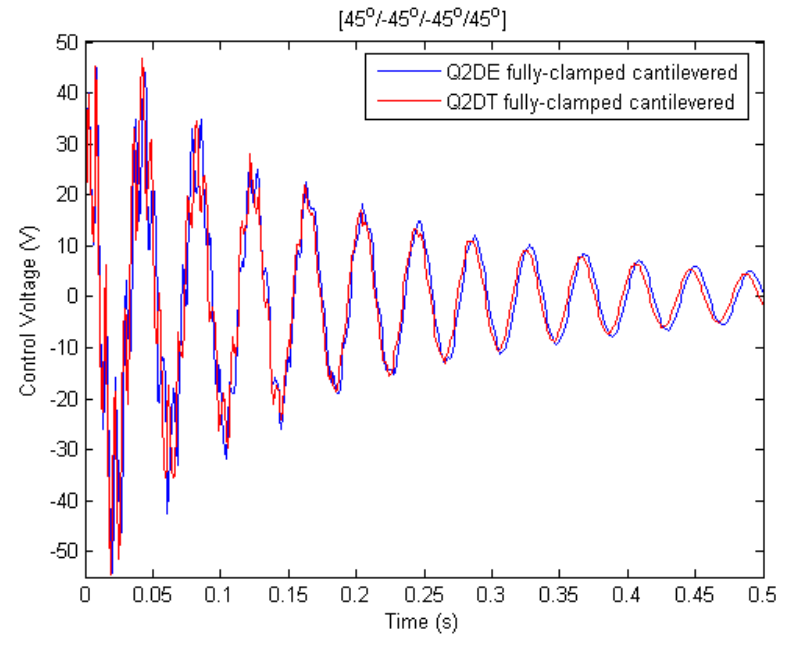

(c)

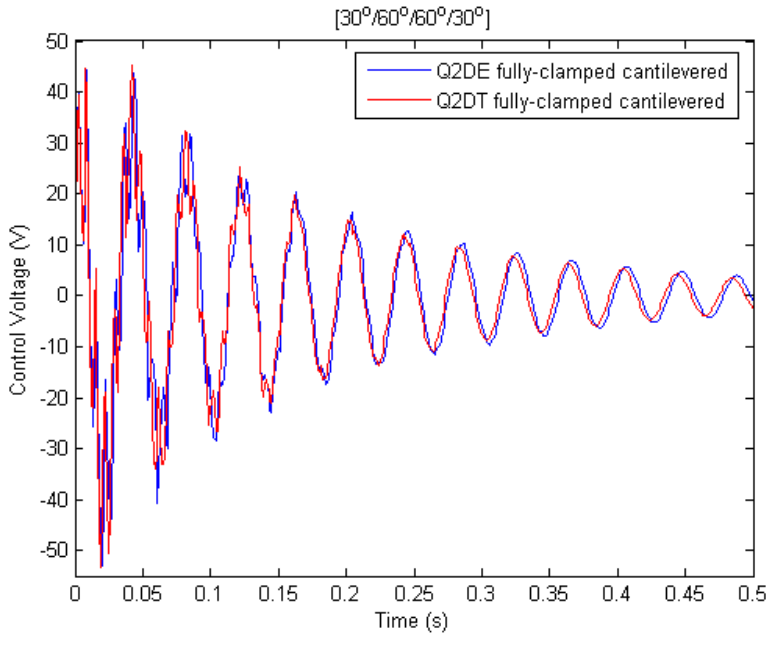

$(d)$

Figure 5.18: Effect of ply orientation on the actuator voltage for controlled-active-passive damping of fully-clamped cantilevered beam. (a) $0_{4}^{\circ}$, (b) $\left[0^{\circ} / 90^{\circ}\right]_{S}$, (c) $\left[45^{\circ} /-45^{\circ}\right]_{S}$, (d) $\left[30^{\circ} / 60^{\circ}\right]_{S}$. 


\subsubsection{Partially-clamped Cantilevered Beam Case}

A partially-clamped cantilevered beam is partially-clamped at the left end and free at the right end as depicted in Figure 5.19. The clamped component is the host beam whereas the viscoelastic and PFRC layers are unconstrained.

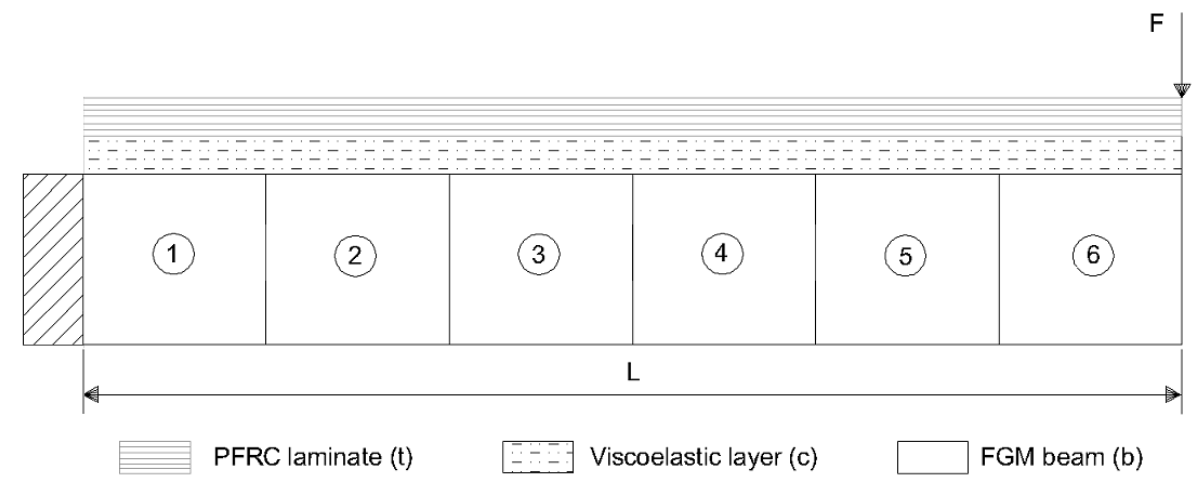

Figure 5.19: Partially-clamped cantilevered beam with ACLD treatment.

Similar to the fully-clamped cantilevered beam case, the gain $\left(K_{v}=350 \mathrm{~V} /(\mathrm{m} / \mathrm{s})\right)$ and geometric parameters remain the same. The simulation time is also unchanged at $0.5 \mathrm{sec}-$ onds. With respect to ply arrangements and stiffness, the same observations are made and are depicted in Figures 5.20, 5.21, and 5.22. The major difference between the fully- and partially- clamped cantilevered cases arises in the peak-to-peak amplitudes of vibration. It is apparent that the partially-clamped case results in higher vibration amplitudes than the fully-clamped case due to the increased flexibility. A comparison between the fully- and partially- clamped cantilevered responses is further demonstrated and discussed in the next subsection. 


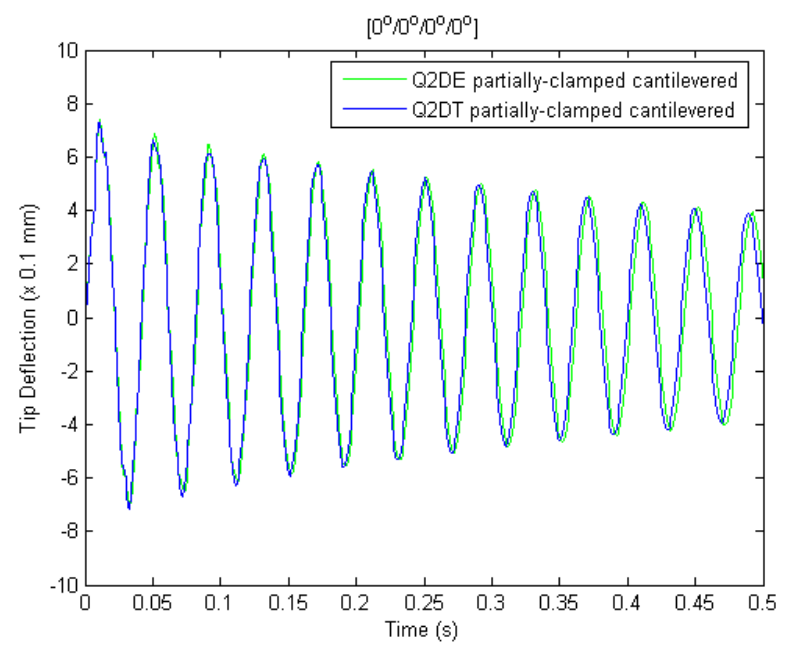

(a)

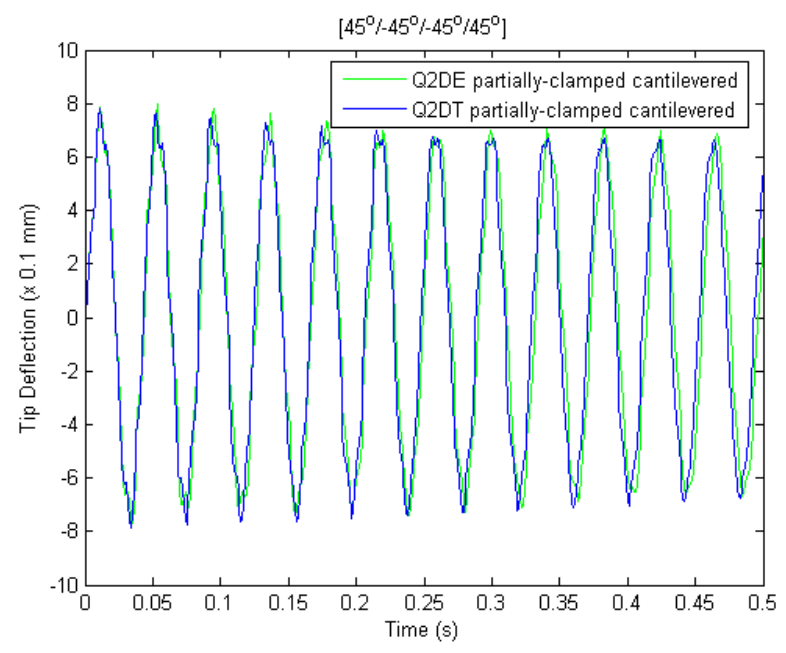

(c)

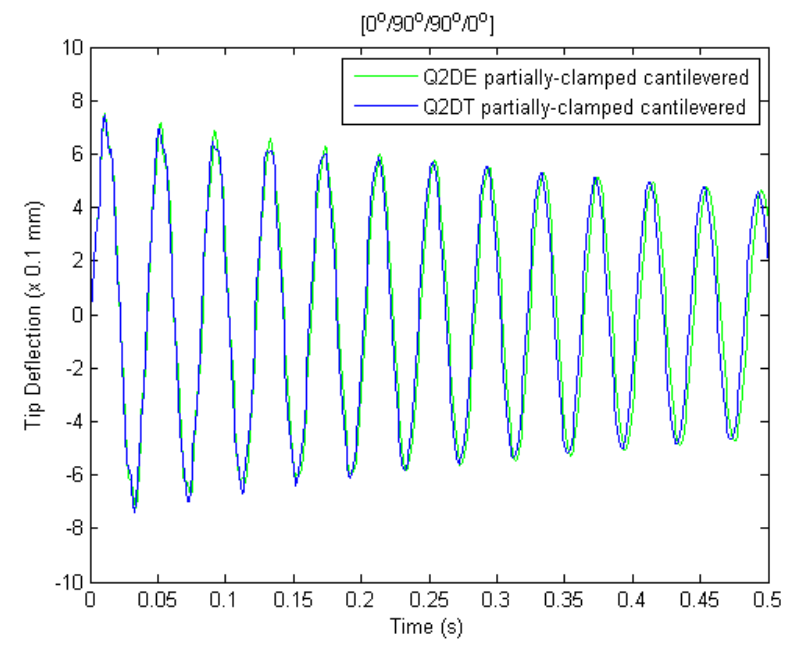

(b)

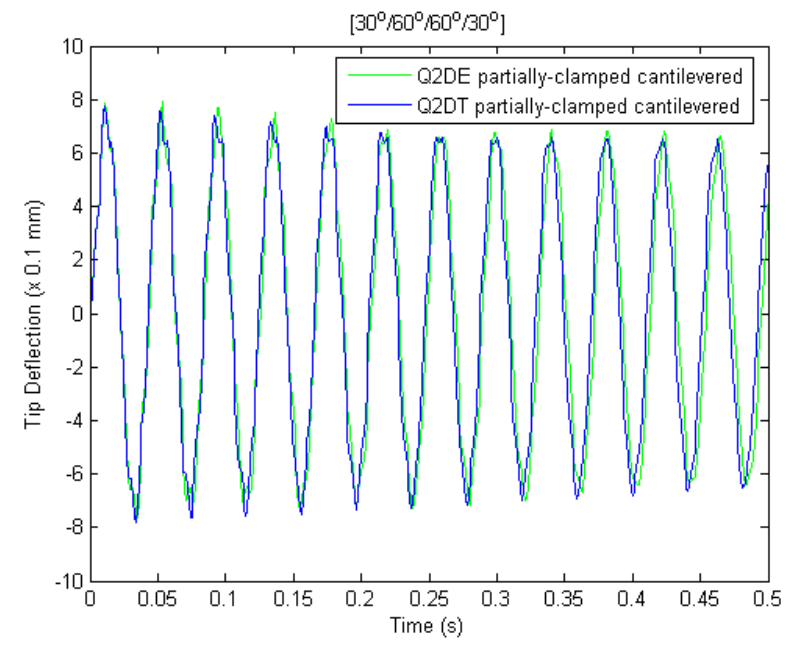

(d)

Figure 5.20: Effect of ply orientation on the tip transverse displacement for uncontrolledpassive damping of partially-clamped cantilevered beam. (a) $0_{4}^{\circ}$, (b) $\left[0^{\circ} / 90^{\circ}\right]_{S}$, (c) $\left[45^{\circ} /-45^{\circ}\right]_{S}$, (d) $\left[30^{\circ} / 60^{\circ}\right]_{S}$. 


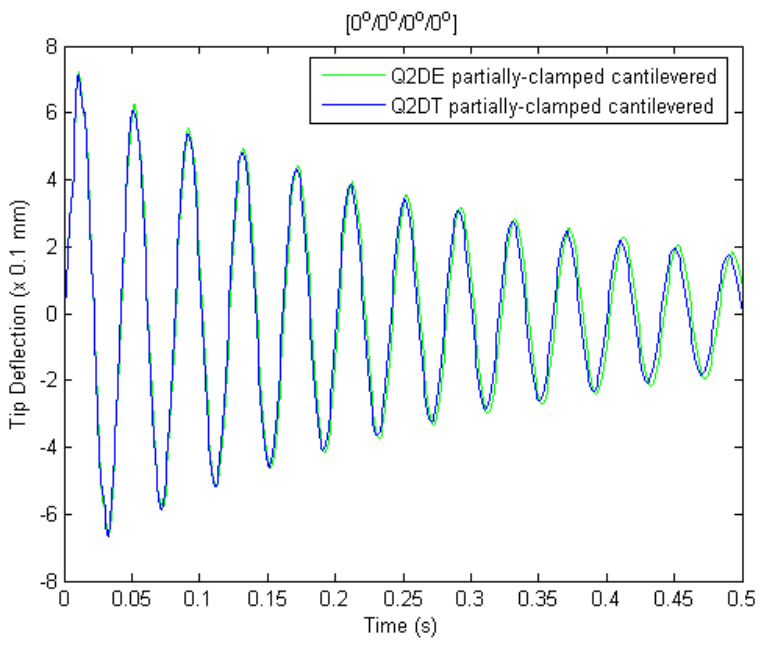

(a)

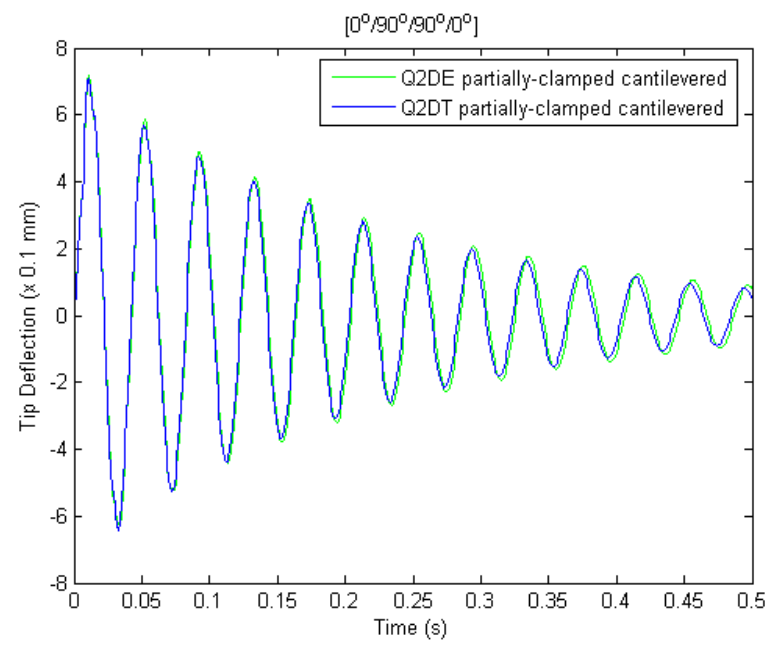

(b)

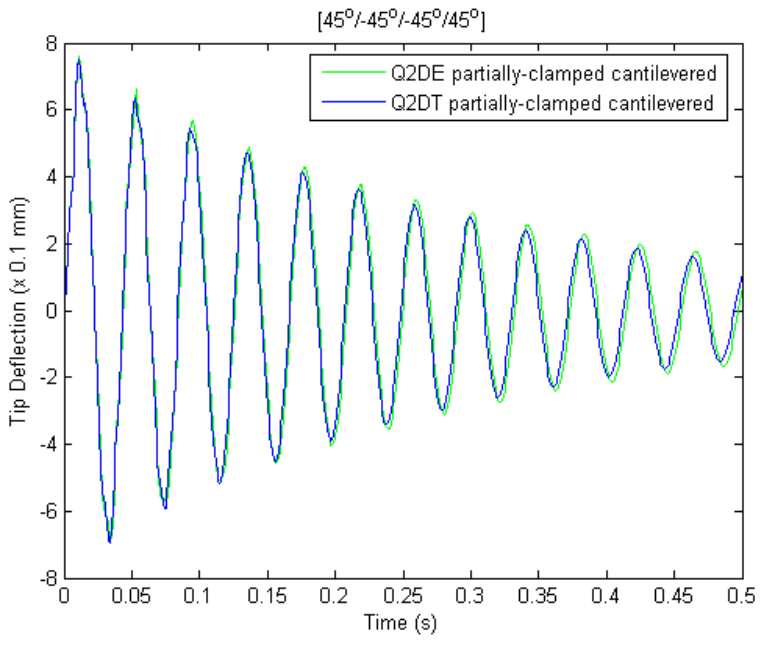

(c)

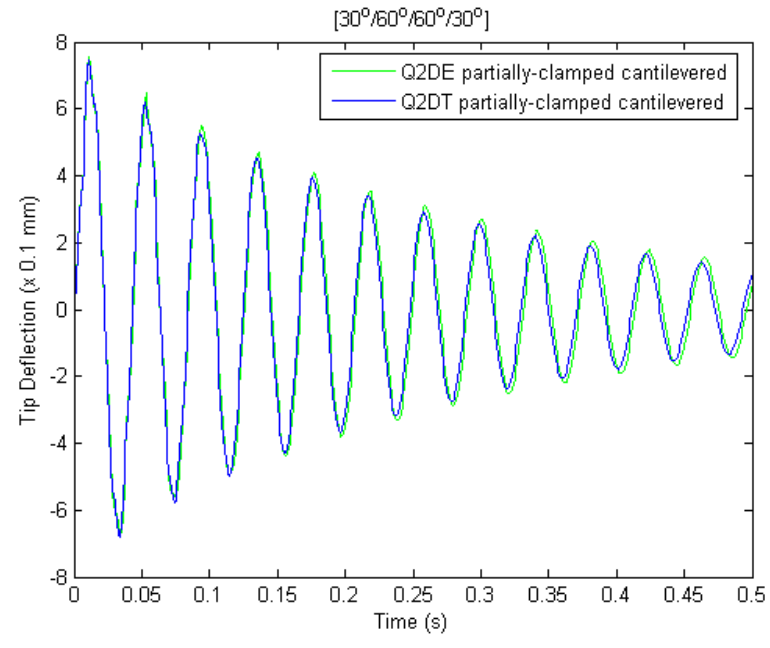

(d)

Figure 5.21: Effect of ply orientation on the tip transverse displacement for controlled-activepassive damping of partially-clamped cantilevered beam. (a) $0_{4}^{\circ}$, (b) $\left[0^{\circ} / 90^{\circ}\right]_{S}$, (c) $\left[45^{\circ} /-45^{\circ}\right]_{S}$, (d) $\left[30^{\circ} / 60^{\circ}\right]_{S}$. 


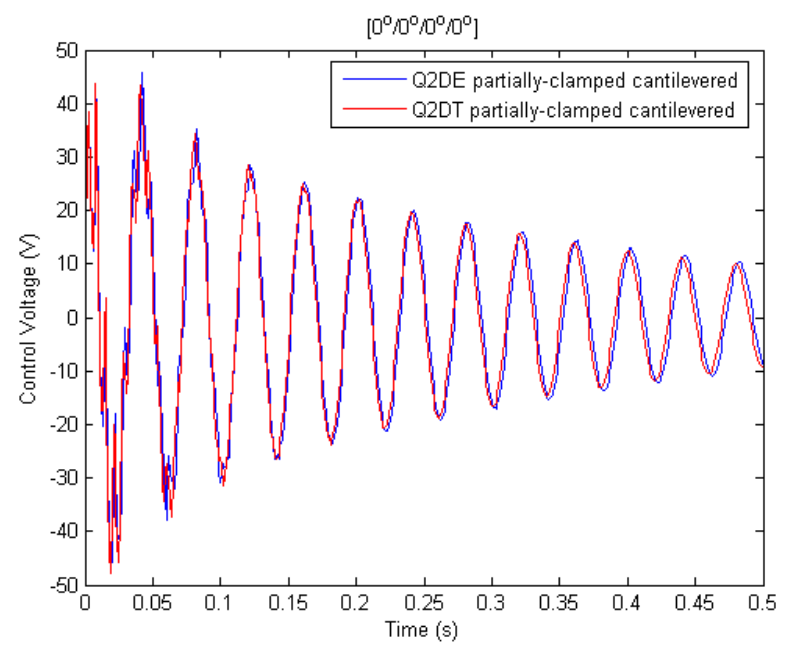

(a)

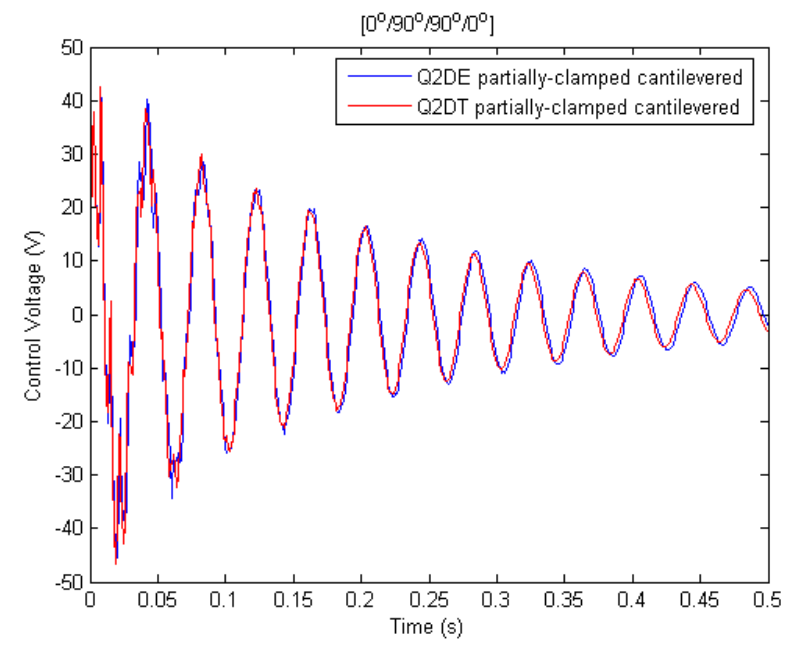

(b)

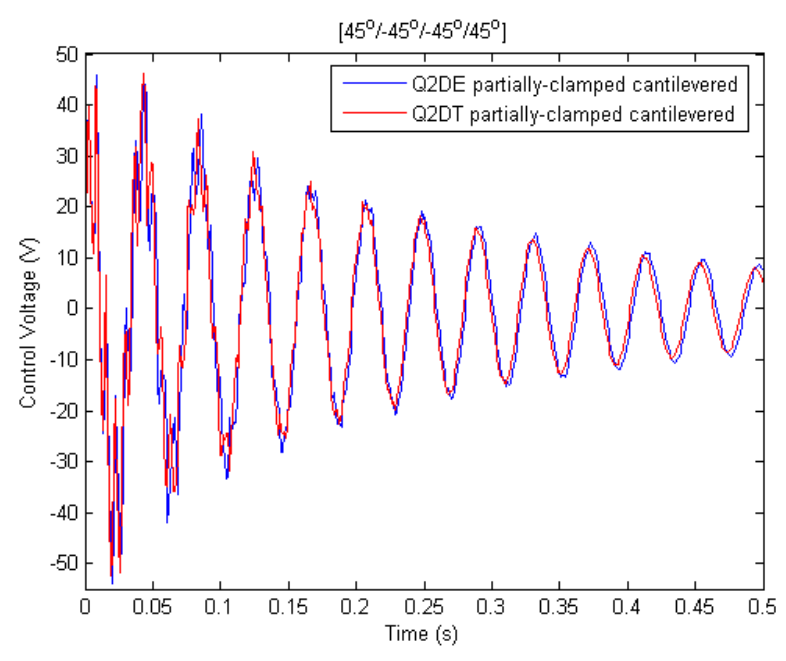

(c)

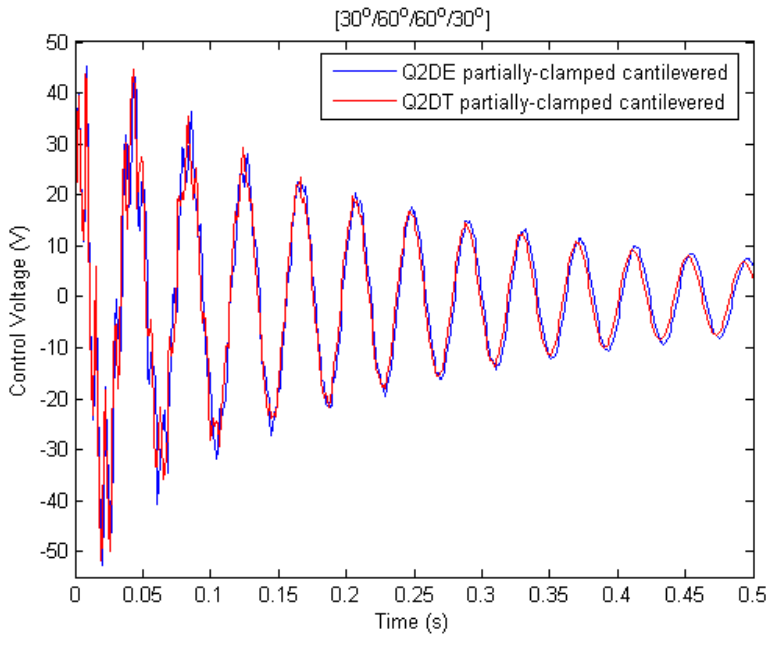

$(d)$

Figure 5.22: Effect of ply orientation on the actuation voltage for controlled-active-passive damping of partially-clamped cantilevered beam. (a) $0_{4}^{\circ}$, (b) $\left[0^{\circ} / 90^{\circ}\right]_{S}$, (c) $\left[45^{\circ} /-45^{\circ}\right]_{S}$, (d) $\left[30^{\circ} / 60^{\circ}\right]_{S}$. 


\subsubsection{Comparison of Fully- and Partially- Clamped Cantilevered with Classi- cal Method}

Figures 5.23, 5.24, and 5.25 depict a comparison of the fully- and partially- clamped cantilevered as well as the classical responses for uncontrolled and controlled damping. It is observed from the uncontrolled damping tip displacement responses in Figure 5.23 that the classical and Q2DT fully-clamped cantilevered cases have very similar responses, with a small phase shift from one another. The $0_{4}^{\circ}$ ply arrangement has partially-clamped cantilevered responses that are higher in amplitude and has a lagging phase shift from the classical and fully-clamped cases. The differences are possibly due to the stiffness of the $0_{4}^{\circ}$ PFRC. The $\left[0^{\circ} / 90^{\circ}\right]_{S}$ PFRC, perhaps due to its reduced stiffness, has a smaller amplitude difference ( the partially-clamped amplitude to the classical and fully-clamped cantilevered amplitudes) than the the $0_{4}^{\circ}$ PFRC. On the other hand, the $\left[45^{\circ} /-45^{\circ}\right]_{S}$ and $\left[30^{\circ} / 60^{\circ}\right]_{S}$ PFRC's resulted in very similar responses for all three (classical, partially- and fully- clamped cantilever) methods, due to its moderate flexibility.

Now, with the introduction of a $350 \mathrm{~V} /(\mathrm{m} / \mathrm{s})$ gain controller, the response is controlled and dampens according to its stiffness (see Figure 5.24). For all four ply arrangements, the Q2DT fully-clamped cantilevered response results in the least amount of vibration compared to the partially-clamped cantilevered and classical method. The $\left[0^{\circ} / 90^{\circ}\right]_{S}$ PFRC gives the best control. Also, the $0_{4}^{\circ}$ PFRC is the only arrangement with its partially-clamped cantilevered amplitude of vibration greater than that of the classical method. Due to the high stiffness of the $0_{4}^{\circ}$ PFRC, the partially-clamped cantilevered (unconstrained PFRC) does not have as much control as those that are more flexible.

With respect to the voltage actuation, Figure 5.25, its response resembles that of the controlled deflection response, as indicated in Figure 5.24, due to their linear relationship. 


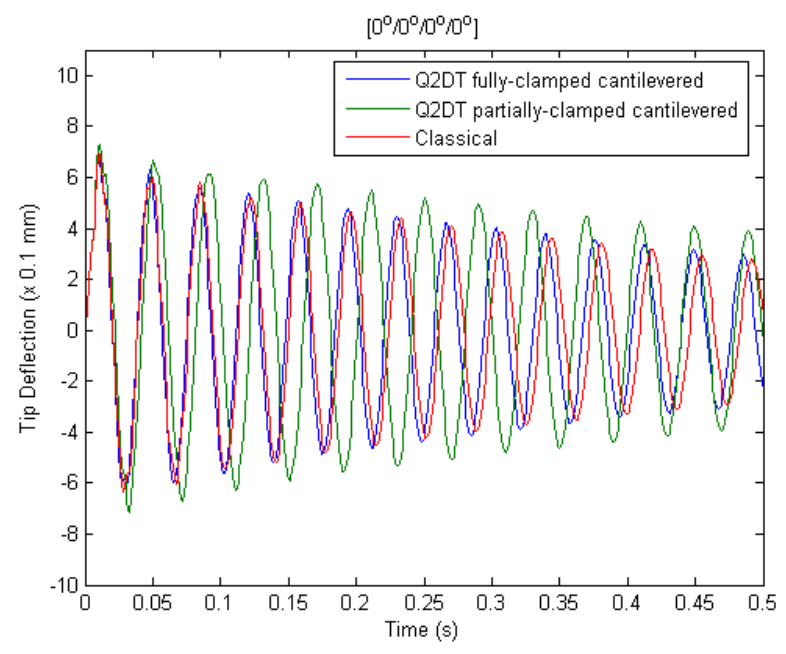

(a)

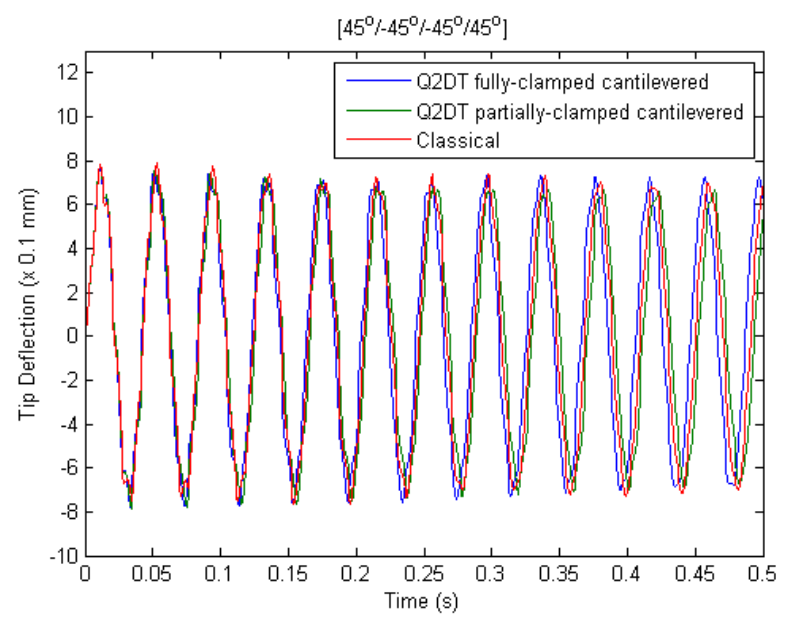

(c)

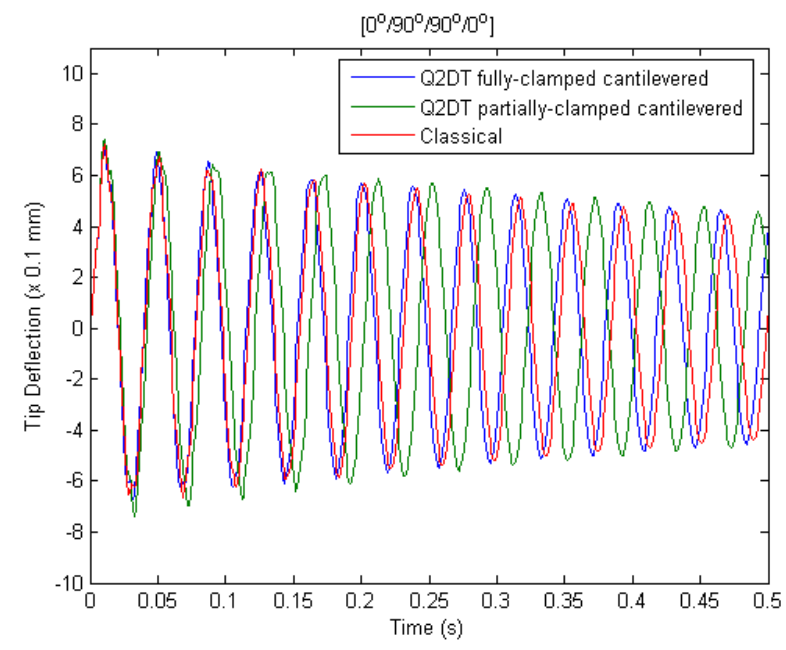

(b)

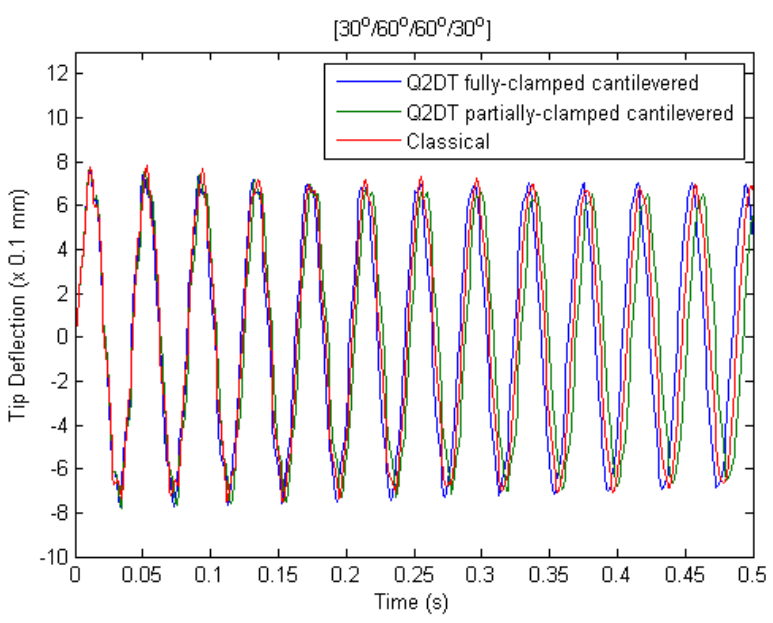

(d)

Figure 5.23: Comparison of tip transverse displacements for uncontrolled-passive fully- and partially- clamped cantilevered beams. (a) $0_{4}^{\circ}$, (b) $\left[0^{\circ} / 90^{\circ}\right]_{S}$, (c) $\left[45^{\circ} /-45^{\circ}\right]_{S}$, (d) $\left[30^{\circ} / 60^{\circ}\right]_{S}$. 


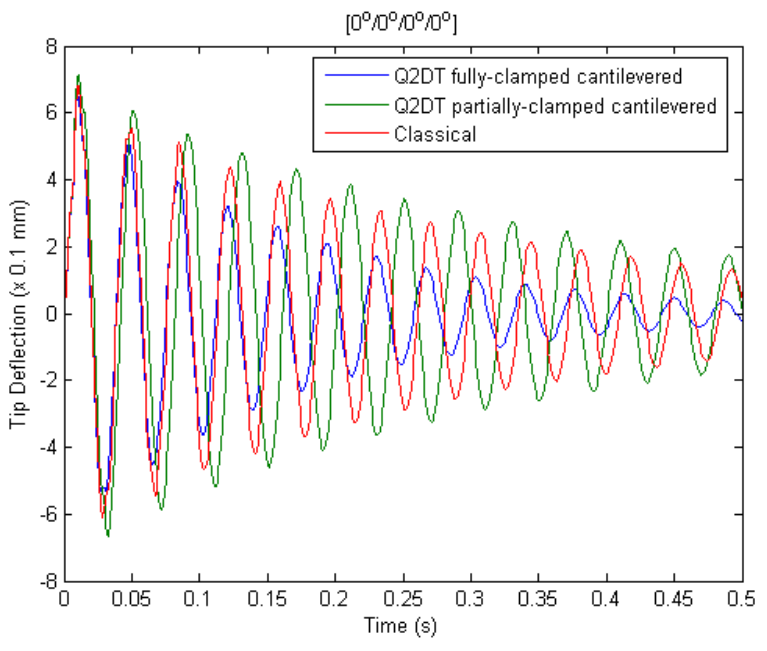

(a)

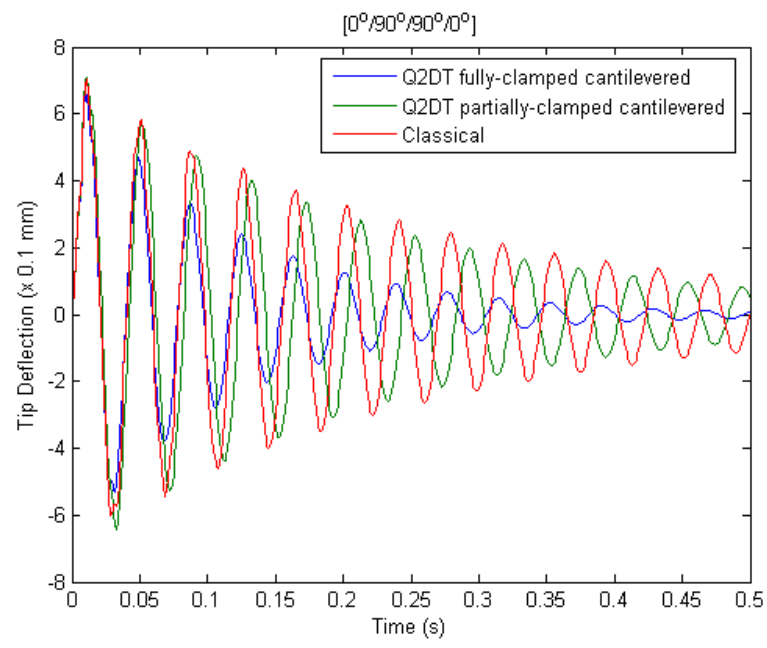

(b)

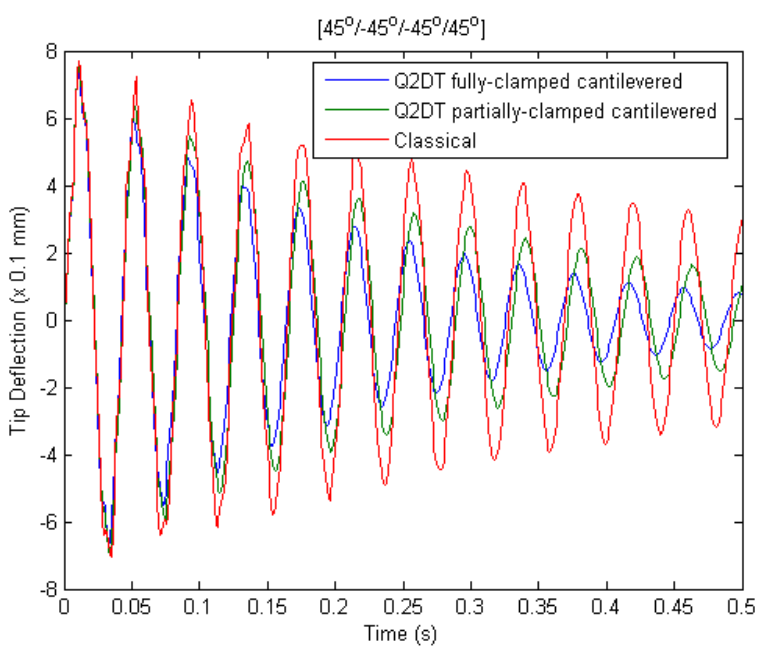

(c)

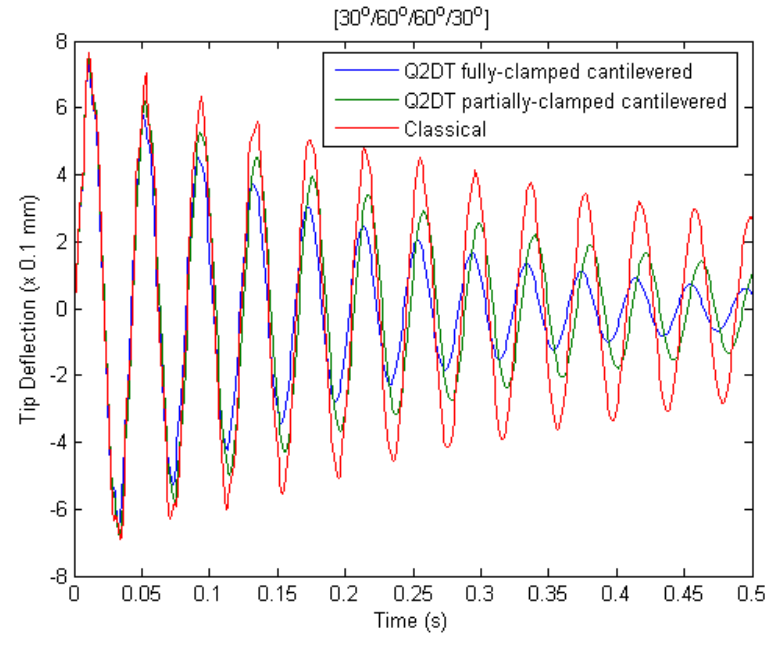

$(d)$

Figure 5.24: Comparison of tip transverse displacements for controlled-active-passive fullyand partially- clamped cantilevered beams. (a) $0_{4}^{\circ}$, (b) $\left[0^{\circ} / 90^{\circ}\right]_{S}$, (c) $\left[45^{\circ} /-45^{\circ}\right]_{S}$, (d) $\left[30^{\circ} / 60^{\circ}\right]_{S}$. 


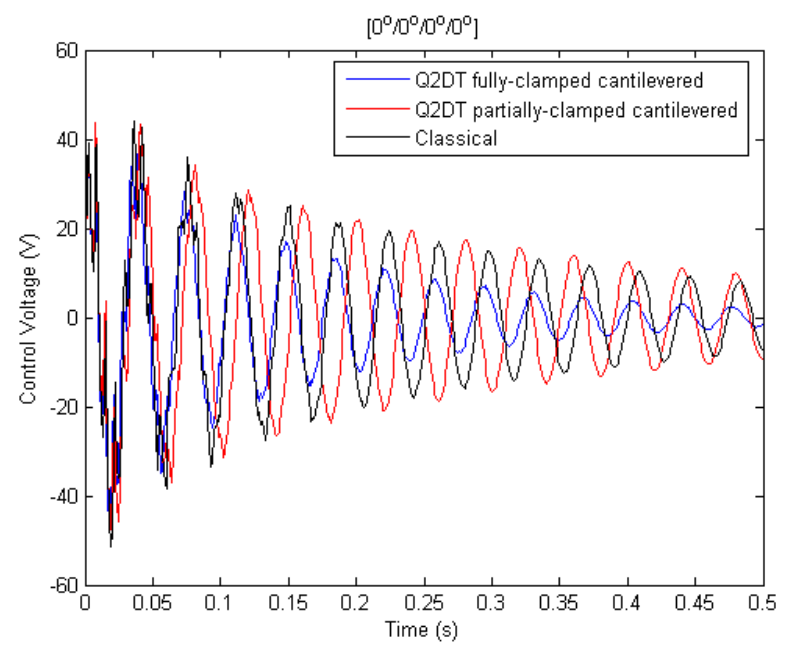

(a)

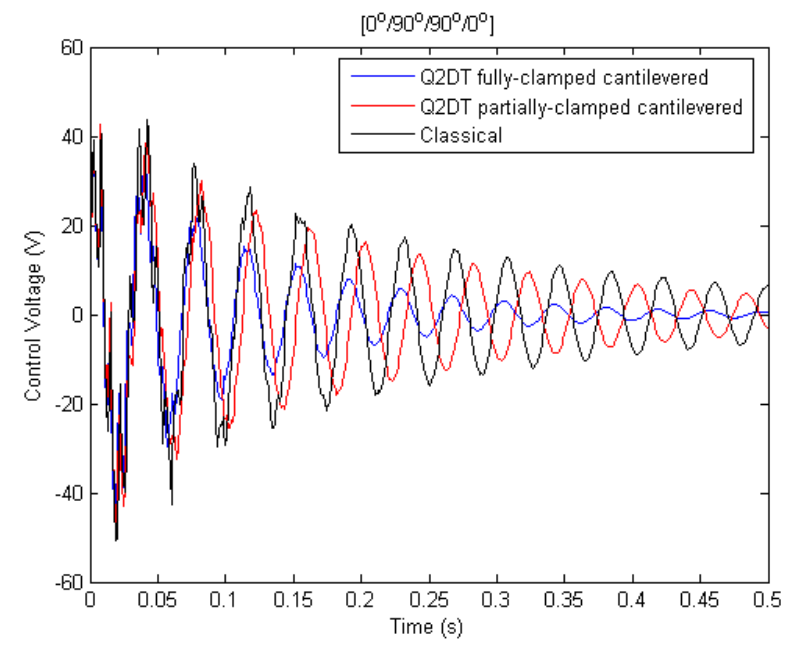

(b)

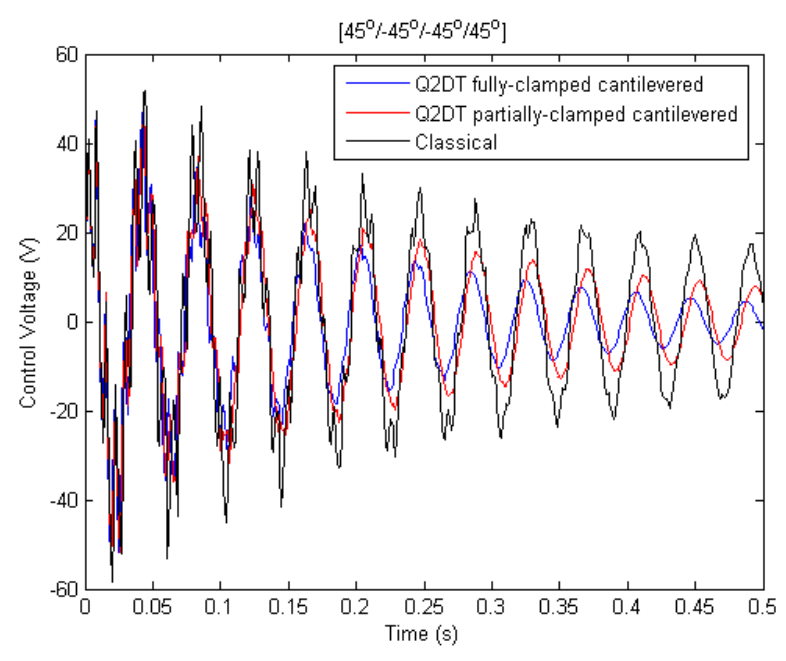

(c)

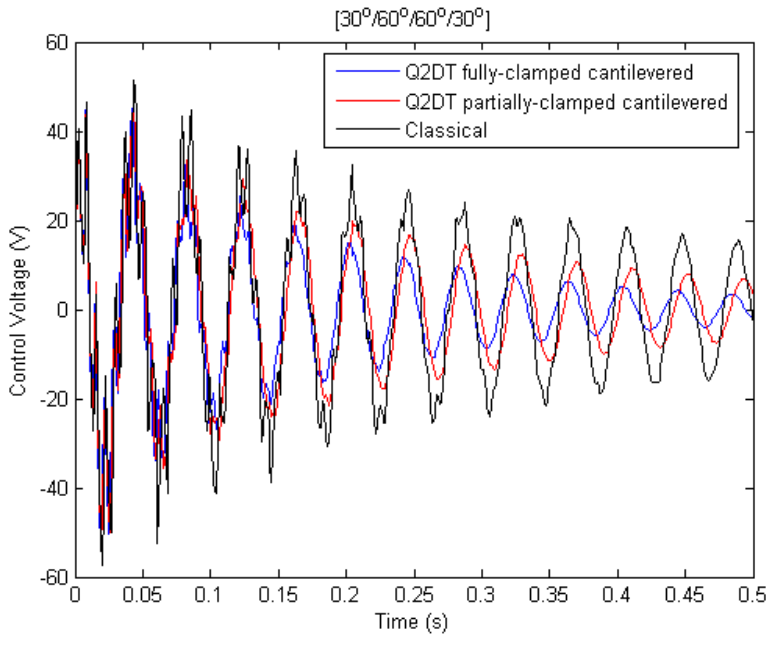

$(d)$

Figure 5.25: Comparison of actuation voltages for controlled-active-passive fully- and partially- clamped cantilevered beams. (a) $0_{4}^{\circ}$, (b) $\left[0^{\circ} / 90^{\circ}\right]_{S}$, (c) $\left[45^{\circ} /-45^{\circ}\right]_{S}$, (d) $\left[30^{\circ} / 60^{\circ}\right]_{S}$. 


\subsubsection{Partially Clamped-clamped Beam Case}

In order to take advantage of the actuation of the PRFC laminate, the host beam is the only component that is clamped at each end. The triangular impulse load, as seen in the second part of Figure 5.14, is applied in the transverse direction to the middle upper node of the top PFRC layer (shared between elements 3 and 4), as depicted in Figure 5.26.

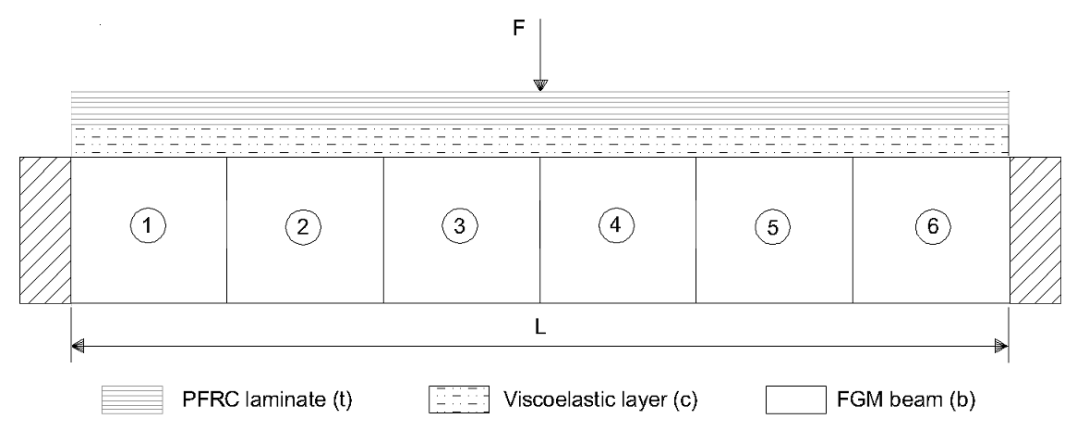

Figure 5.26: Partially clamped-clamped beam with ACLD treatment.

The same gain as in both the cantilevered beam cases, $K_{v}=350 \mathrm{~V} /(\mathrm{m} / \mathrm{s})$, is utilized in this instance. All parameter specifications remain the same except for the host beam thickness and the time period, which is reduced to $h_{b}=2.0 \mathrm{~mm}$ from $3.0 \mathrm{~mm}$ and 0.25 seconds from 0.5 seconds, respectively. Note that the parameter modifications were made solely for the enhancement of the presentation. As a result of the boundary conditions, the response for the partially clamped-clamped should have higher frequency of vibration as well as smaller transverse displacement compared to the cantilevered beam cases.

The deflection of interest is that of the middle node of the ACLD beam in the transverse direction. The passive and active-passive damping responses are depicted in Figures 5.27 and 5.28, respectively. Figure 5.29 is the actuator voltage plots that complement the displacement responses. As in the cantilevered beam cases, the same PFRC arrangements are analyzed. The partially clamped-clamped beam case exhibits the same response pattern as the cantilevered beam examples. The passive $0_{4}^{\circ}$ PFRC response in Figure 5.27 results in the best damping, which follows the same reasoning as the fully-clamped cantilevered beam case. Further, the $\left[0^{\circ} / 90^{\circ}\right]_{S}$ PFRC is found to be the most controlled among the active-passive damping response. This is due to the enhancement of actuation control in a more flexible PFRC laminate, as depicted in Figure 5.28. The $\left[45^{\circ} /-45^{\circ}\right]_{S}$ PFRC plot shows the worst damping response, since the flexibility of the PFRC laminate is not increased in the bending direction. The actuation voltage responses, seen in Figure 5.29, are directly proportional to 
the derivative of the controlled damping responses in Figure 5.28 .

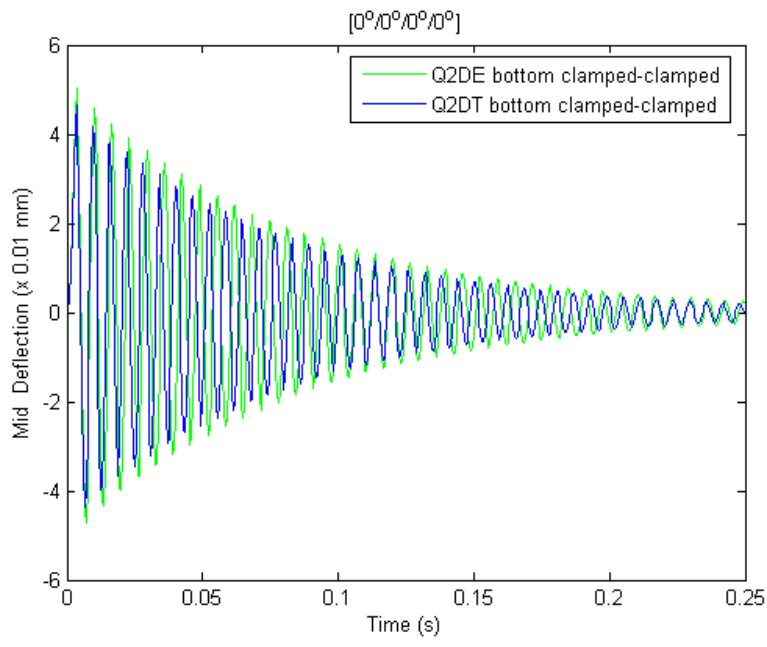

(a)

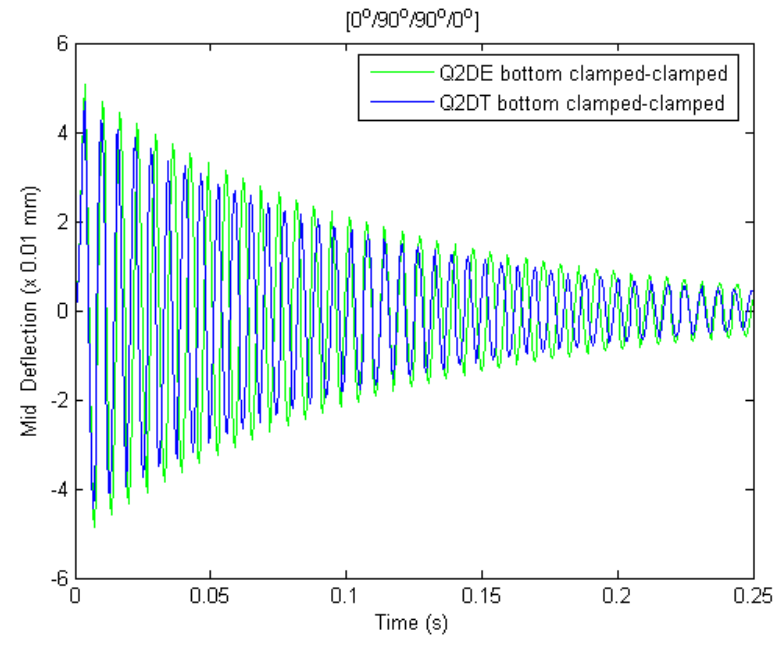

(b)

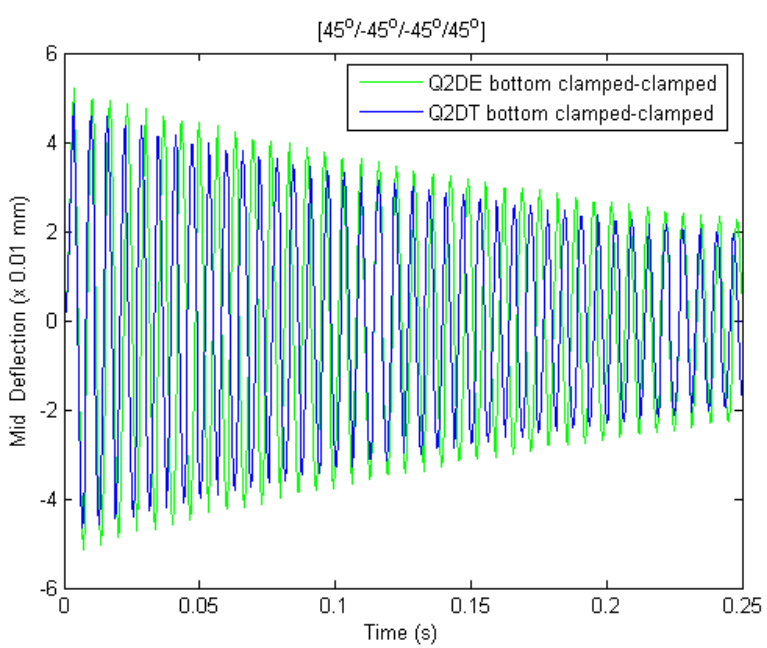

$(c)$

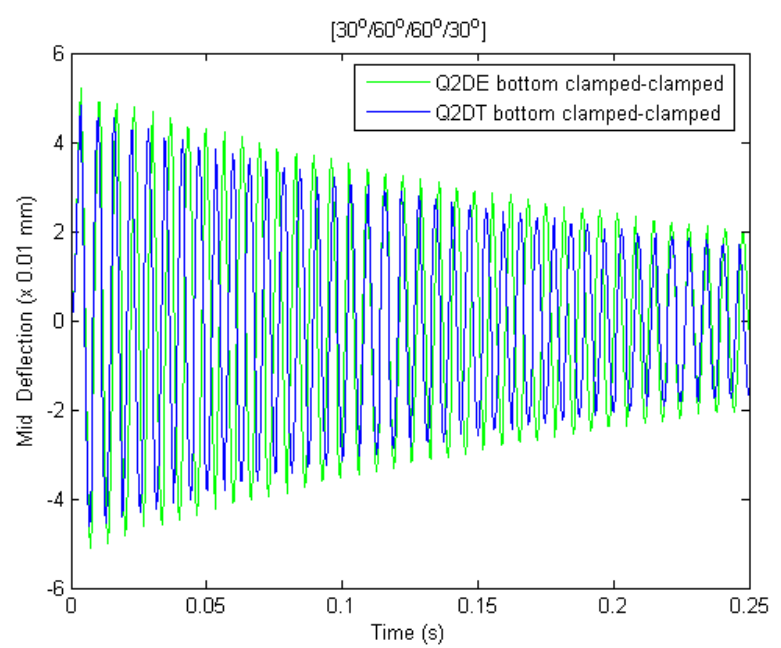

$(d)$

Figure 5.27: Effect of ply orientation on the mid-point transverse displacement for uncontrolled-passive damping of partially (bottom) clamped-clamped beam. (a) $0_{4}^{\circ}$, (b) $\left[0^{\circ} / 90^{\circ}\right]_{S}$, (c) $\left[45^{\circ} /-45^{\circ}\right]_{S}$, (d) $\left[30^{\circ} / 60^{\circ}\right]_{S}$. 


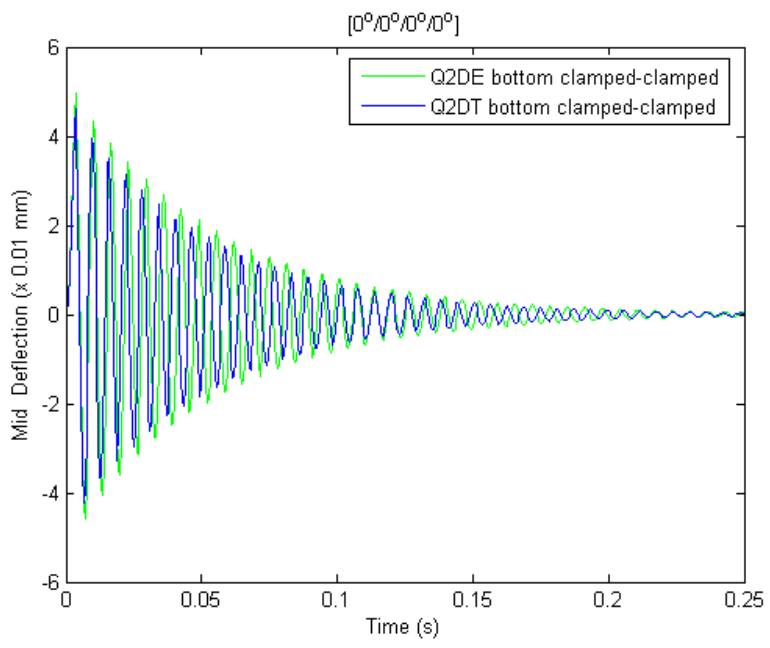

(a)

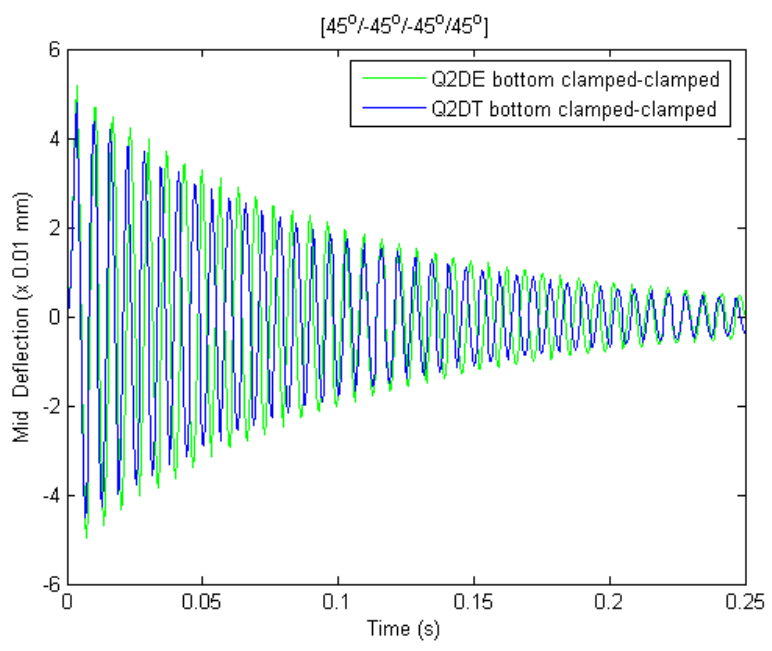

(c)

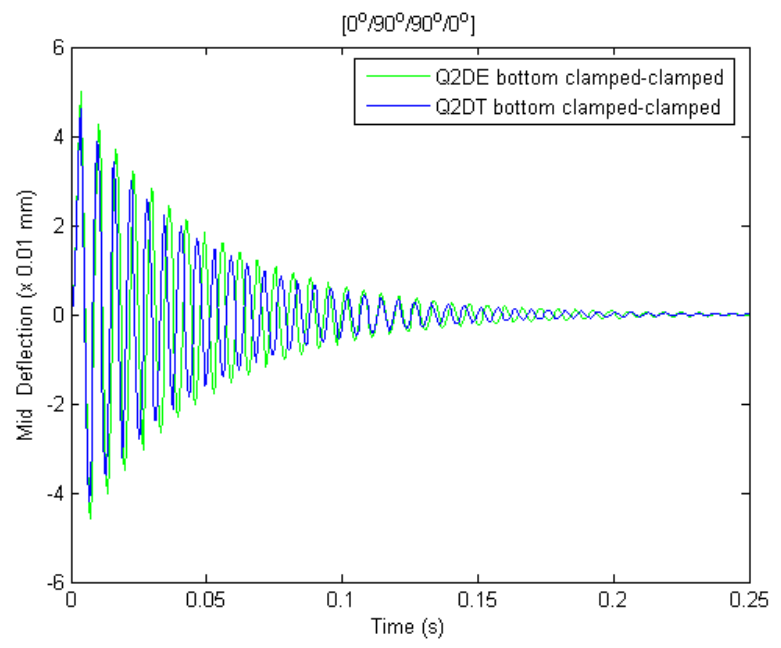

(b)

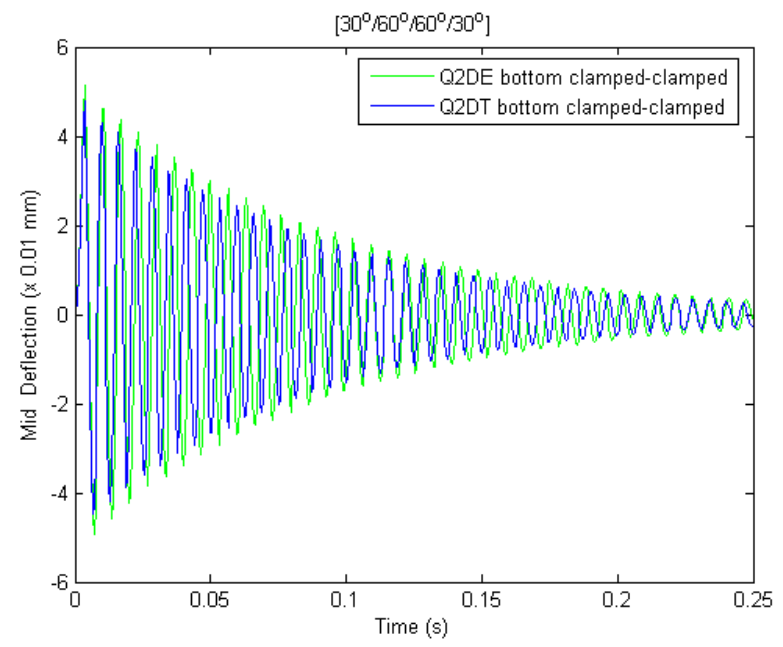

$(d)$

Figure 5.28: Effect of ply orientation on the mid-point transverse displacement for controlledactive-passive damping of partially (bottom) clamped-clamped beam. (a) $0_{4}^{\circ}$, (b) $\left[0^{\circ} / 90^{\circ}\right]_{S}$, (c) $\left[45^{\circ} /-45^{\circ}\right]_{S},(\mathrm{~d})\left[30^{\circ} / 60^{\circ}\right]_{S}$. 


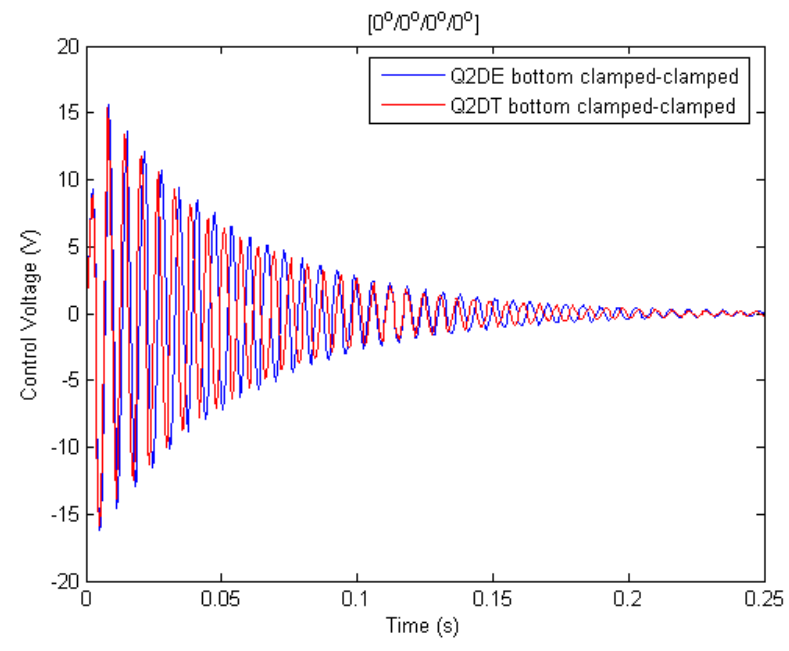

(a)

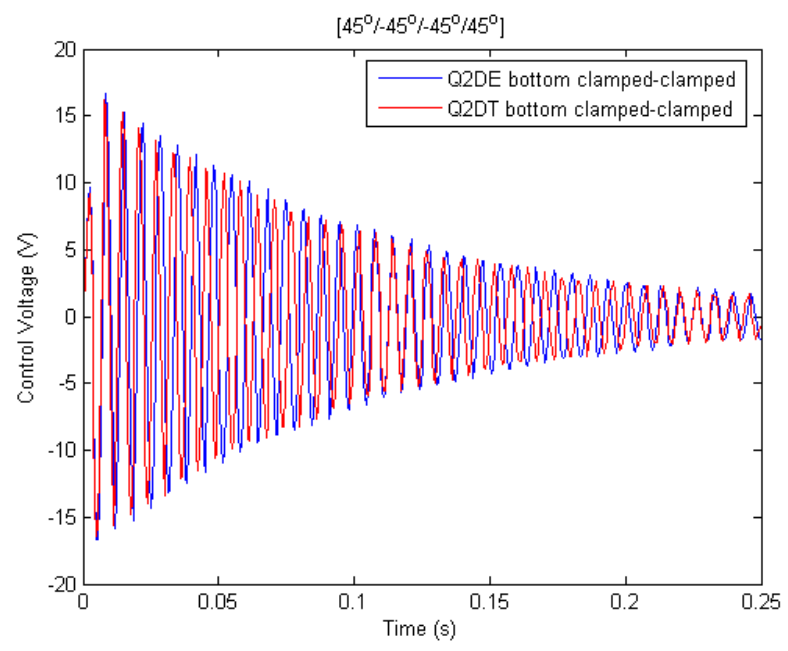

(c)

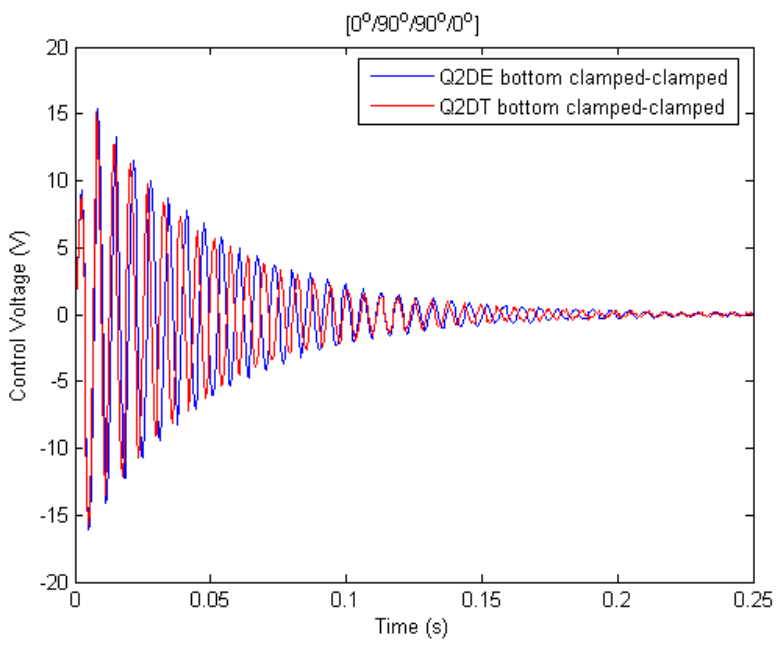

(b)

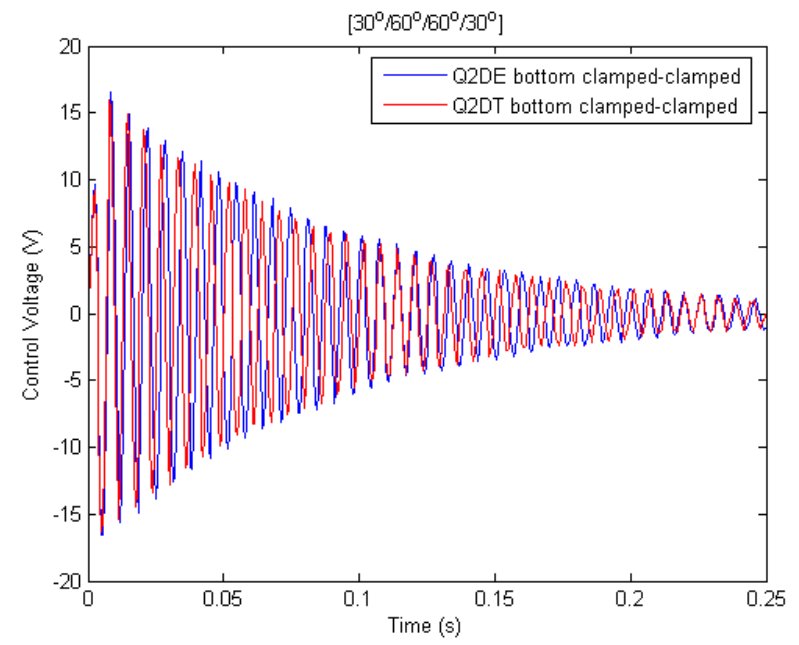

$(d)$

Figure 5.29: Effect of ply orientation on the actuation voltage for controlled-active-passive damping of partially (bottom) clamped-clamped beam. (a) $0_{4}^{\circ}$, (b) $\left[0^{\circ} / 90^{\circ}\right]_{S}$, (c) $\left[45^{\circ} /-45^{\circ}\right]_{S}$, (d) $\left[30^{\circ} / 60^{\circ}\right]_{S}$. 


\subsubsection{Simply-supported Beam Case}

The configuration of the simply-supported boundary condition consists of the support of two corner nodes, each at the ends of the beam, as depicted in Figure 5.30. These support are constrained in both the axial and transverse displacements.

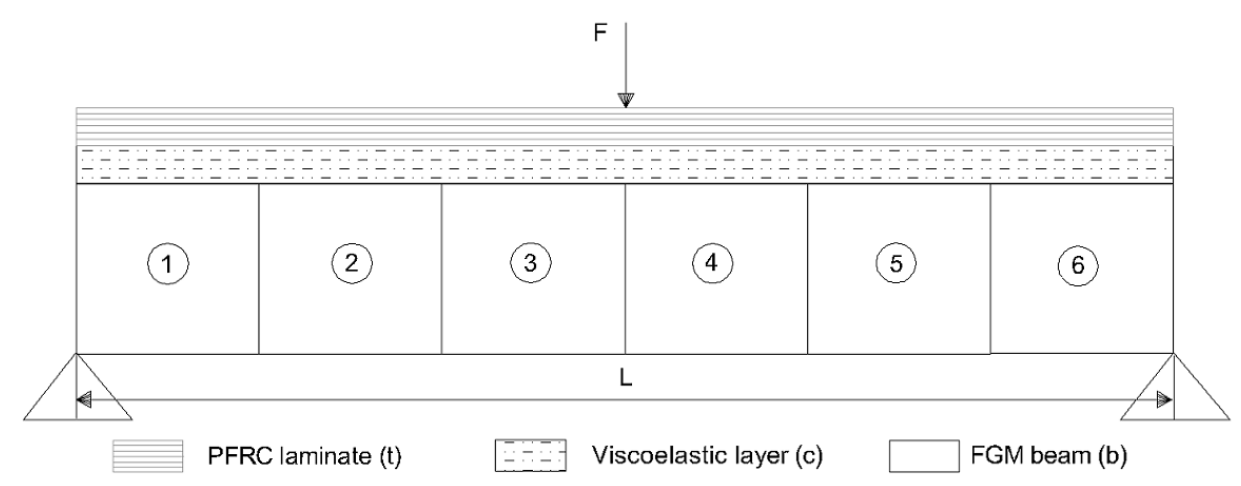

Figure 5.30: Simply-supported beam with ACLD treatment.

Similar to the partially clamped-clamped beam case, the gain, host beam thickness, and observation time period are $K_{v}=350 \mathrm{~V} /(\mathrm{m} / \mathrm{s}), h_{b}=2.0 \mathrm{~mm}$, and $\mathrm{t}=0.25$ seconds, respectively. Also, the same triangular impulse load is applied to the middle node in the transverse direction.

Figures 5.31, 5.32, and 5.33 illustrate the uncontrolled-passive damping, active-passive damping, and actuator voltage of the simply-supported beam, respectively. The deflection of the simply-supported beam is observed to be much higher than the partially clampedclamped beam (almost 1.5 times). This observation is plausible and it is attributed to the increased flexibility of the beam.

The same four PFRC arrangements are examined as in the previous cases. Similar to the other cases, the passive $0_{4}^{\circ}$ PFRC arrangement in Figure 5.31 shows the fastest damping while the $\left[45^{\circ} /-45^{\circ}\right]_{S}$ and $\left[30^{\circ} / 60^{\circ}\right]_{S}$ PFRC's have very slow attenuation rates due to their lower stiffnesses. Also, from Figure 5.32 , the controlled-active-passive response that has the best damping response is, once again, the $\left[0^{\circ} / 90^{\circ}\right]_{S}$ PFRC. 


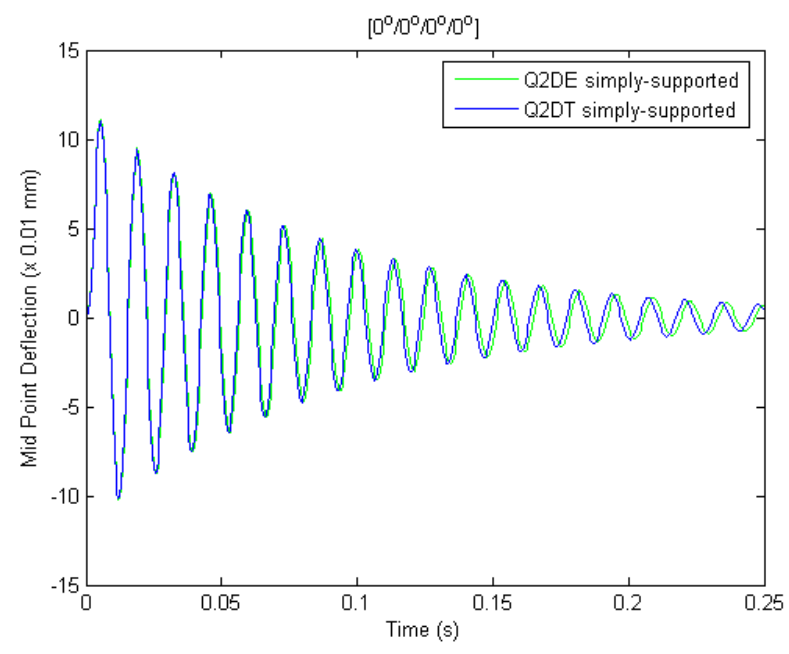

(a)

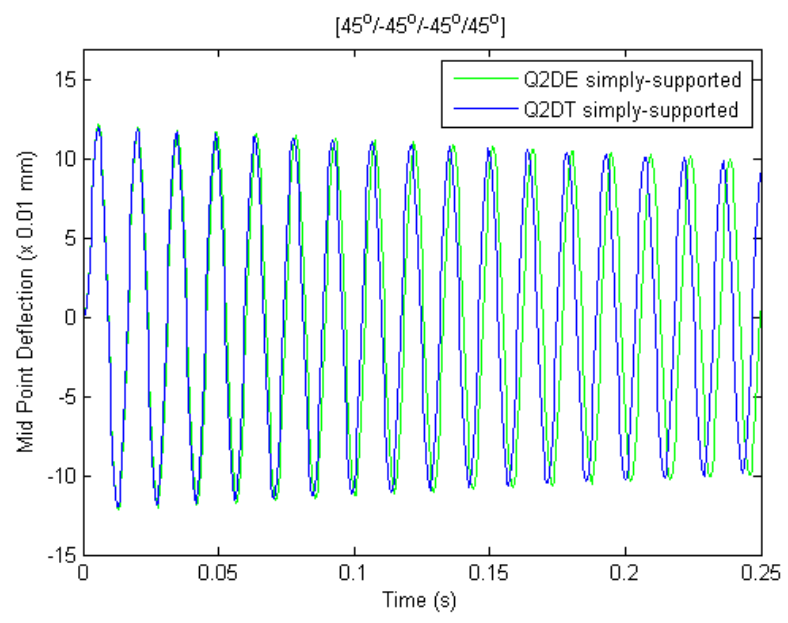

(c)

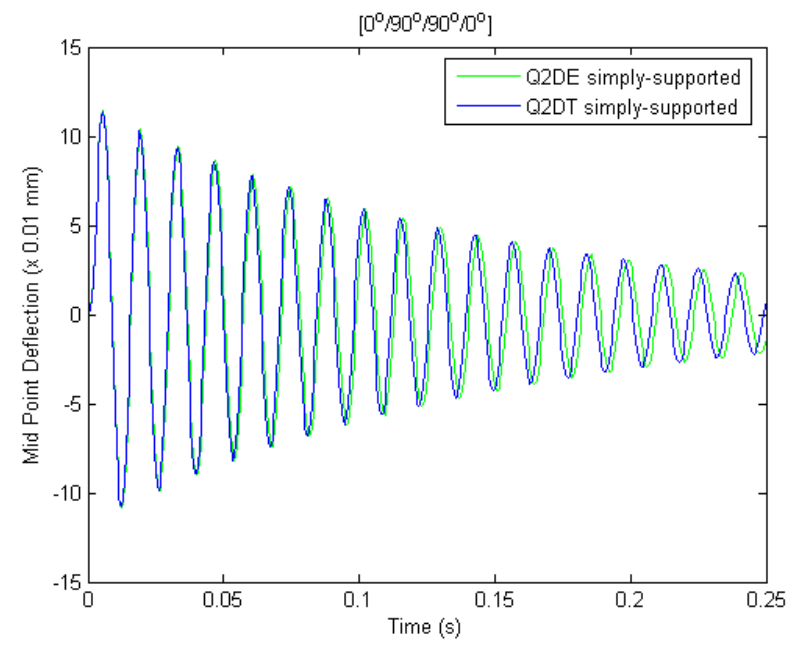

(b)

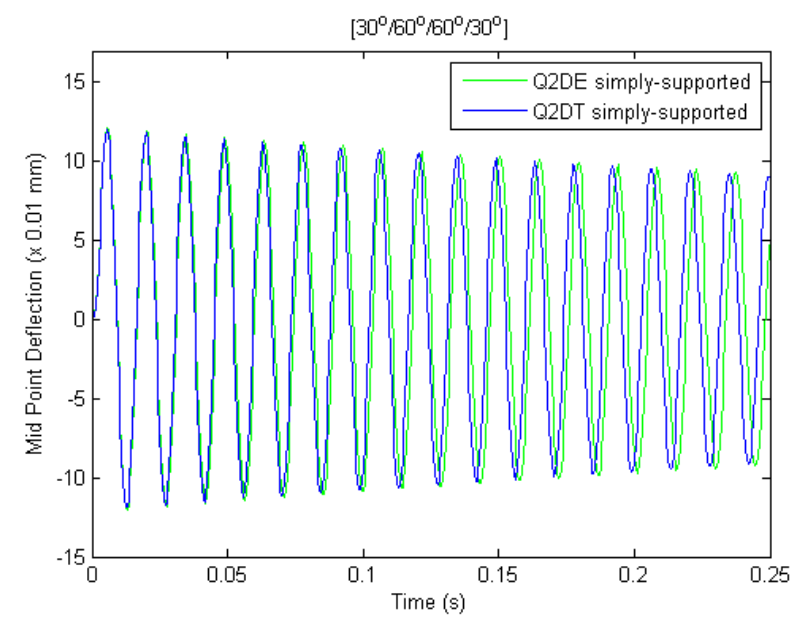

$(d)$

Figure 5.31: Effect of ply orientation on the mid-point transverse displacement for uncontrolled-passive damping of simply-supported beam. (a) $0_{4}^{\circ}$, (b) $\left[0^{\circ} / 90^{\circ}\right]_{S}$, (c) $\left[45^{\circ} /-45^{\circ}\right]_{S}$, (d) $\left[30^{\circ} / 60^{\circ}\right]_{S}$. 


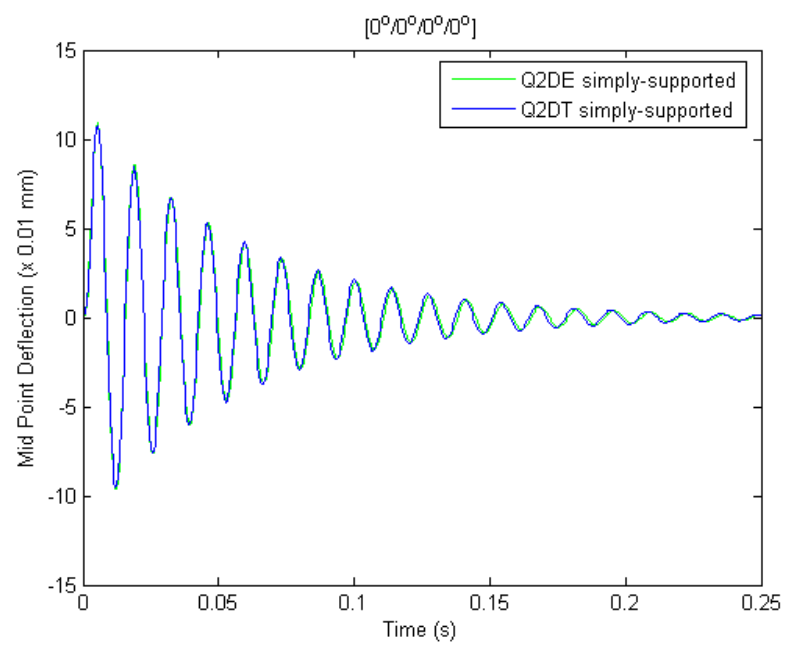

(a)

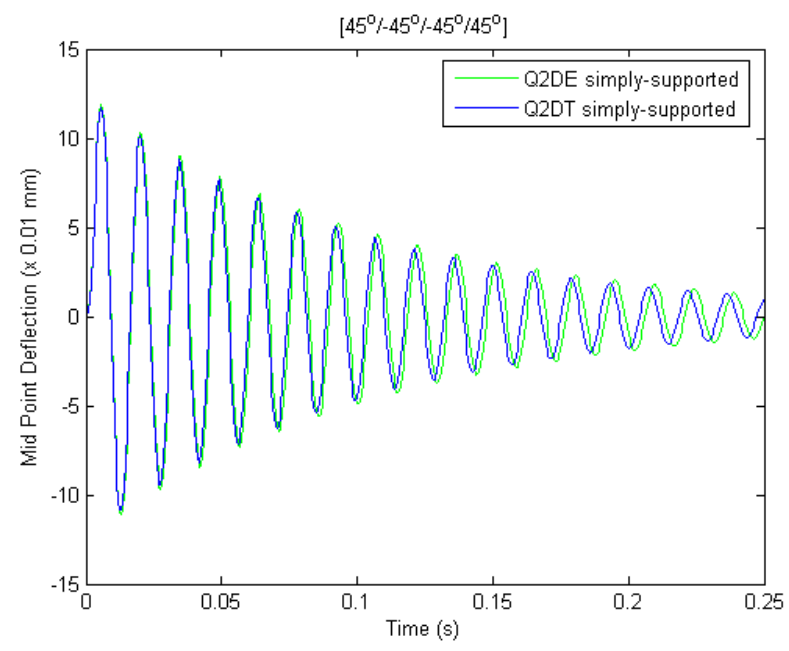

(c)

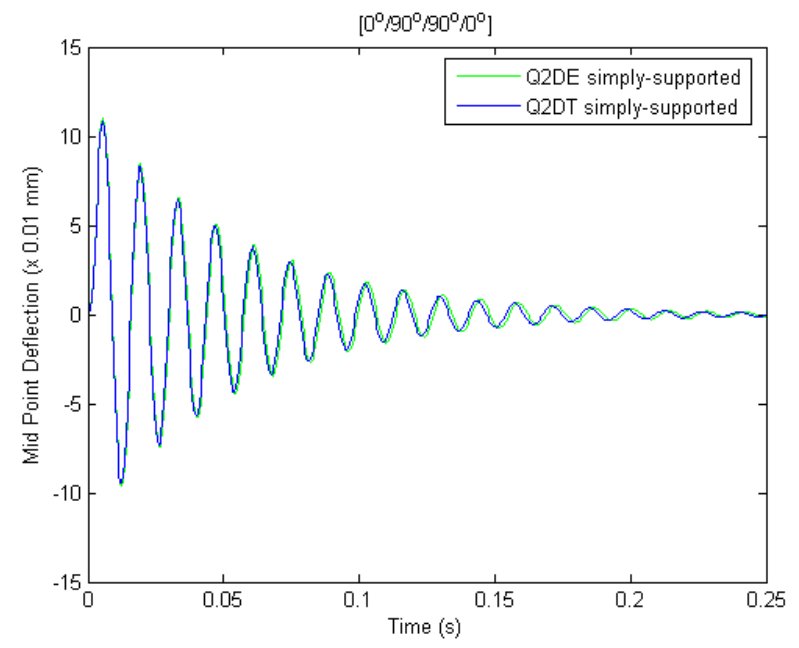

(b)

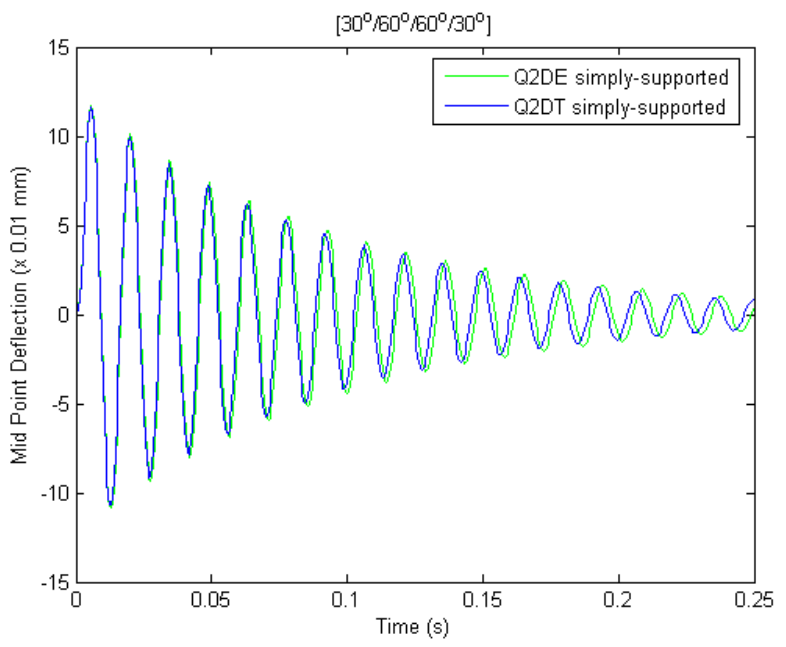

$(d)$

Figure 5.32: Effect of ply orientation on the mid-point transverse displacement for controlledactive-passive damping of simply-supported beam. (a) $0_{4}^{\circ}$, (b) $\left[0^{\circ} / 90^{\circ}\right]_{S}$, (c) $\left[45^{\circ} /-45^{\circ}\right]_{S}$, (d) $\left[30^{\circ} / 60^{\circ}\right]_{S}$. 


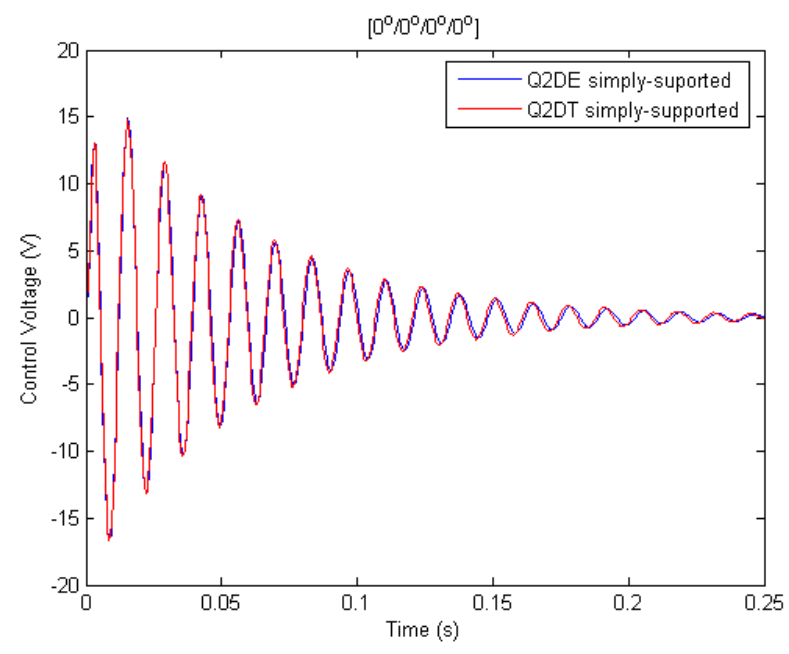

(a)

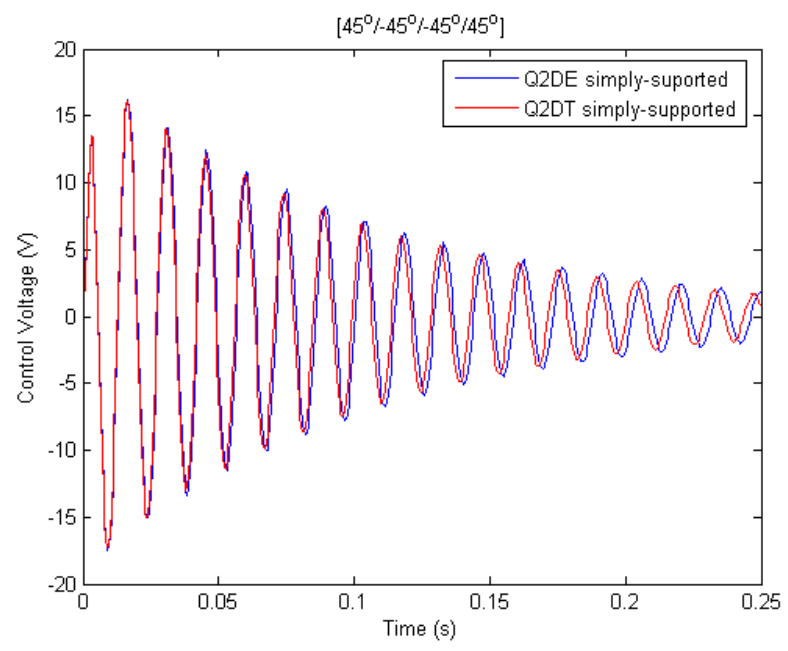

(c)

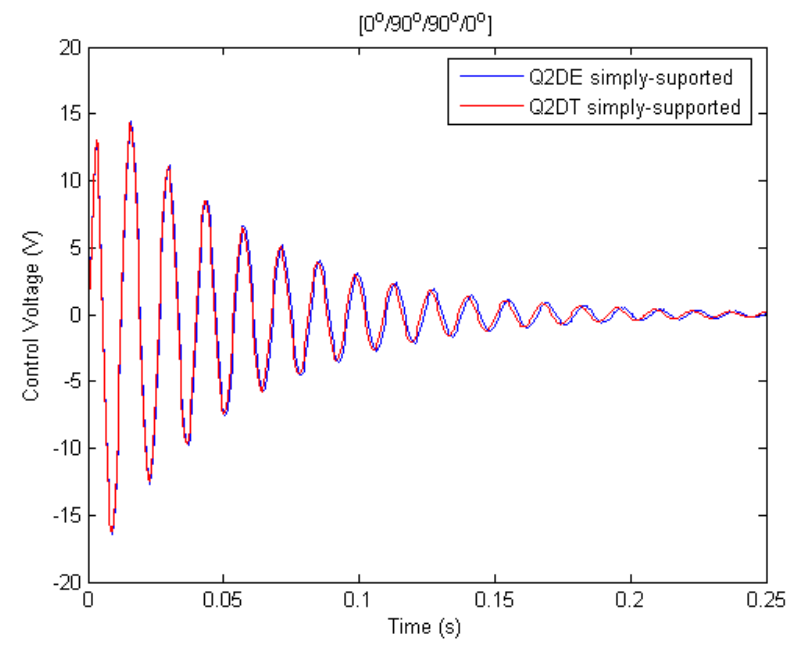

(b)

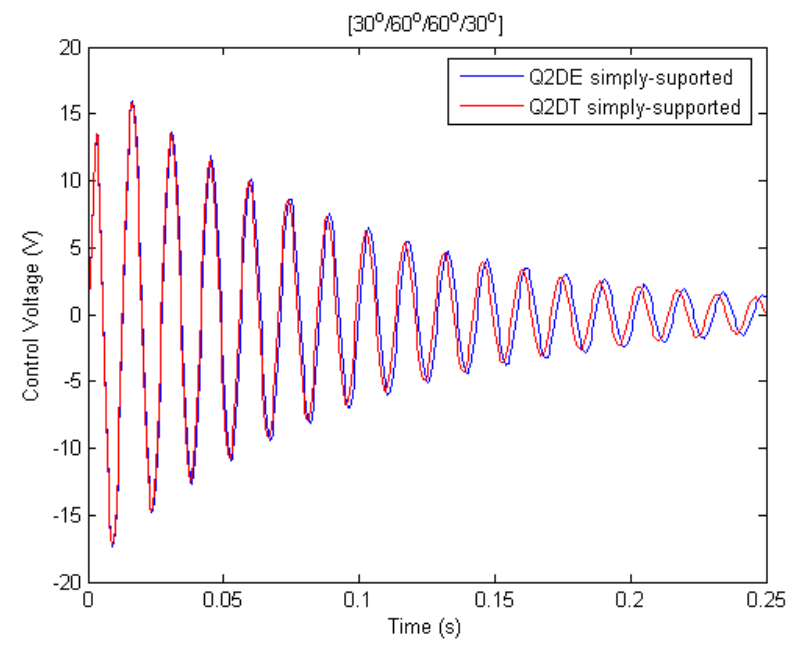

$(d)$

Figure 5.33: Effect of ply orientation on the actuation voltage for controlled-active-passive damping of simply-supported beam. (a) $0_{4}^{\circ}$, (b) $\left[0^{\circ} / 90^{\circ}\right]_{S}$, (c) $\left[45^{\circ} /-45^{\circ}\right]_{S}$, (d) $\left[30^{\circ} / 60^{\circ}\right]_{S}$. 


\subsubsection{Effect of FGM Properties}

In order to study the effect of the functionally graded (FG) property in the host beam, all of the plies in the PFRC laminate are set to $\theta=0^{\circ}$. The material properties of the FGM beam are assumed to change continuously throughout the thickness of the beam, according to the volume fraction of the constituent materials based on the function. In this study, the Young's modulus, E, is analyzed under the power-law function and exponential function. The average stiffness of the host beam either increases or decreases by varying the Young's modulus. The average modulus of elasticity is represented as $E_{\text {avg }}=\frac{1}{2 h} \int_{-h}^{h} E(z) d z$, where $E(z)$ is either the power-law function, Equation 3.9, or exponential function, Equation 3.10 . The constituents are metal and ceramic material, wherein the Young's modulus of each are denoted by $E_{M}$ and $E_{C}$, respectively. The top of the host beam has $100 \%$ metal composition and the bottom has $100 \%$ ceramic composition. Note that only the elastic modulus is analyzed since previous analysis in section 5.2 indicated that the effects of changes in the density and Poisson's ratio are neglible.

Throughout this investigation, all material properties and parameters of the top PFRC, viscoelastic core, and host beam remain the same as that used in Table 5.7, with the exception of the host beam's Young's modulus. The beam problems remain the same, with the parameter specifications as depicted Figure 5.14. Also, the gain remains the same as the previous subsection, $K_{v}=350 \mathrm{~V} /(\mathrm{m} / \mathrm{s})$.

Three scenarios are investigated to study the effect of the FG property in the host beam: (i) the effect of change in average elastic moduli, (ii) the effect of varying constituents moduli for fixed average elastic moduli, and (iii) the effect of exchange in the metal to ceramic and ceramic to metal elastic moduli.

The first analysis is performed by fixing the ceramic elastic modulus, $E_{C}$, and altering the metal elastic modulus, $E_{M}$, in order to have average elastic moduli, $E_{\text {avg }}$, of $200 \mathrm{GPa}$, $230 \mathrm{GPa}$, and $260 \mathrm{GPa}$. The variation of Young's modulus with respect to the change in the average elastic modulus is tabulated in Table 5.8 .

Table 5.8: Variation of Young's modulus with respect to change in average elastic modulus.

\begin{tabular}{ccccccc}
\hline \hline & \multicolumn{6}{c}{ Host Beam Elastic Modulus (GPa) } \\
\cline { 2 - 6 } & \multirow{2}{*}{$E_{M}$} & \multirow{2}{*}{$E_{C}$} & \multirow{2}{*}{$\Delta E$} & \multicolumn{4}{c}{$E_{\text {avg }}$} \\
\cline { 5 - 7 } & & & & $(\mathrm{p})$ & P-FGM & E-FGM \\
\hline $\mathrm{a}$ & 260 & 151 & 109 & 1.2 & 200.55 & 200.59 \\
$\mathrm{~b}$ & 333 & 151 & 182 & 1.3 & 230.13 & 230.13 \\
$\mathrm{c}$ & 412 & 151 & 261 & 1.39 & 260.26 & 260.03 \\
\hline
\end{tabular}


Next, $E_{\text {avg }}$ is fixed to $200 \mathrm{GPa}$ while both the ceramic and metal elastic moduli change accordingly. In order to obtain an elastic modulus average of $200 \mathrm{GPa}$, the ceramic and metal elastic moduli were discretely chosen to fit the $E_{\text {avg }}$ relation. The change in elastic modulus $\Delta E$, from metal to ceramic, continually increases as seen in Table 5.9. The metal and ceramic Young's moduli from the table experiences variations similar to those in Figure 5.34. The P-FGM and E-FGM distributions intersect at the middle of the beam thickness because the $E_{a v g}$ 's are the same.

Table 5.9: Variation of Young's modulus with respect to fixed average elastic modulus $\left(E_{M}>E_{C}\right)$.

\begin{tabular}{ccccccc}
\hline \hline & \multicolumn{6}{c}{ Host Beam Elastic Modulus (GPa) } \\
\cline { 2 - 6 } & $E_{M}$ & $E_{C}$ & $\Delta E$ & \multicolumn{4}{c}{$E_{\text {avg }}$} \\
\cline { 5 - 7 } & & & & $(\mathrm{p})$ & P-FGM & E-FGM \\
\hline $\mathrm{a}$ & 260 & 151 & 109 & 1.2 & 200.55 & 200.59 \\
$\mathrm{~b}$ & 352 & 100 & 252 & 1.52 & 200 & 200.24 \\
$\mathrm{c}$ & 436 & 70 & 366 & 1.815 & 200.02 & 200.09 \\
\hline
\end{tabular}

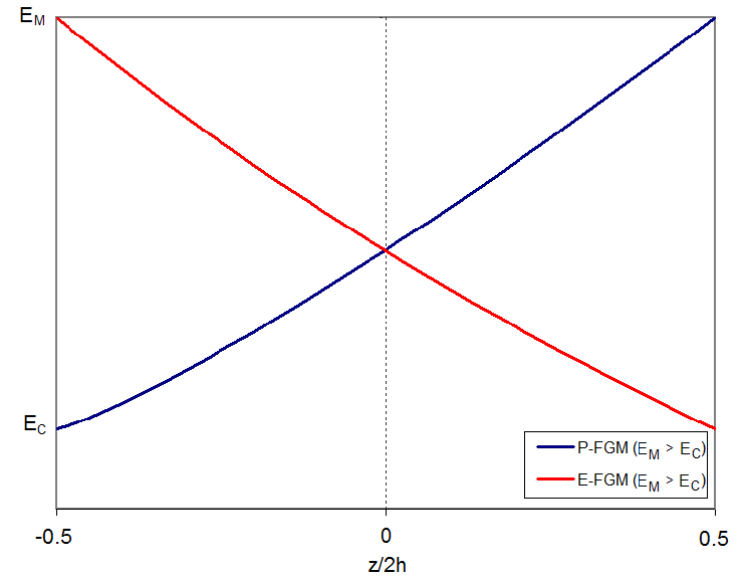

Figure 5.34: Variation of Young's modulus in a P-FGM and E-FGM beam for $E_{M}>E_{C}$.

Finally, the previous data with fixed average elastic moduli are used to exchange the metal to ceramic and ceramic to metal elastic moduli. This exchange demonstrates the effect of selecting the constituents of the FG beam and their placements, either on the top or bottom of the host beam. Table 5.10 shows the values used when exchanging the metal to ceramic and ceramic to metal elastic moduli. The elastic moduli for $E_{M}<E_{C}$ exhibits variations that are similar to those in Figure 5.35 . 
Table 5.10: Exchange of ceramic and metal Young's modulus with respect to fixed average elastic modulus $\left(E_{M}<E_{C}\right)$.

\begin{tabular}{ccccccc}
\hline \hline & \multicolumn{6}{c}{ Host Beam Elastic Modulus (GPa) } \\
\cline { 5 - 7 } & \multirow{2}{*}{$E_{M}$} & $E_{C}$ & $\Delta E$ & \multicolumn{4}{c}{$E_{\text {avg }}$} \\
\cline { 5 - 7 } & & & $(\mathrm{p})$ & P-FGM & E-FGM \\
\hline $\mathrm{a}$ & 151 & 260 & 109 & 0.82 & 200.11 & 200.59 \\
$\mathrm{~b}$ & 100 & 352 & 252 & 0.66 & 200.19 & 200.24 \\
$\mathrm{c}$ & 70 & 436 & 366 & 0.555 & 200.63 & 200.09 \\
\hline
\end{tabular}

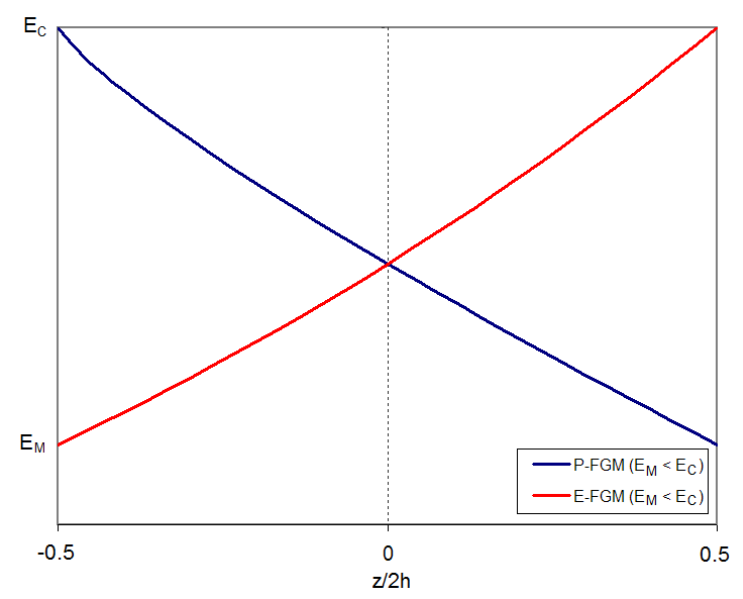

Figure 5.35: Variation of Young's modulus in a P-FGM and E-FGM beam for $E_{M}>E_{C}$.

Figure 5.36 includes the combination of Figures 5.34 and 5.35. By alternating the elastic moduli of the metal and ceramic constituents for the P-FGM and E-FGM functions, they exhibit nearly exact slopes. Since the formulas for both FGM distributions are different, they will not match exactly, but are very close. The reason for this is because the average values were forced to be the same. 


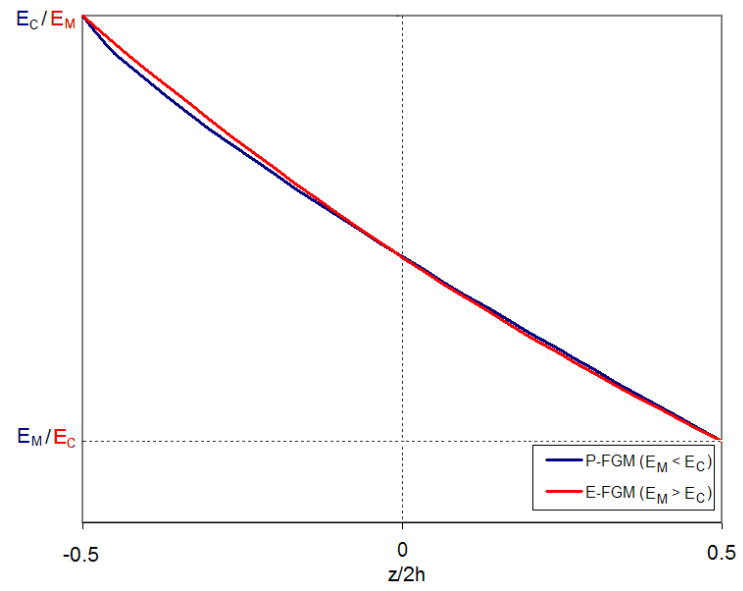

$(a)$

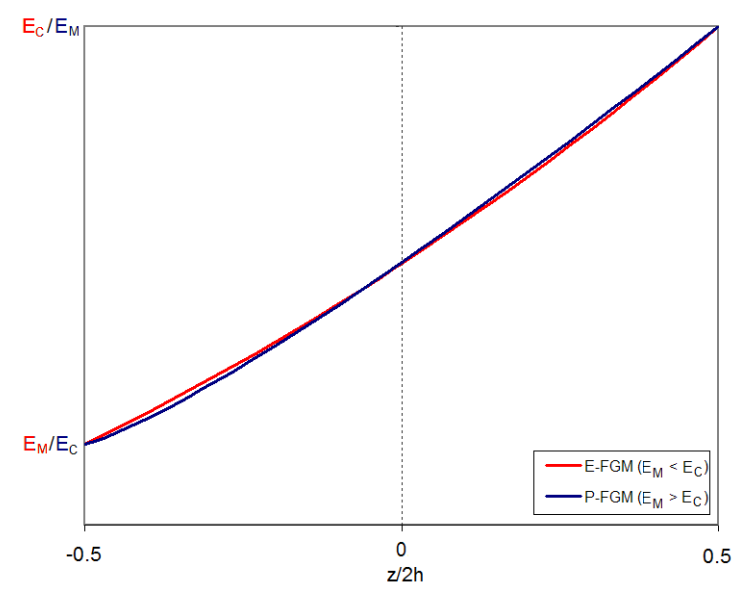

$(b)$

Figure 5.36: Variation of Young's modulus in a (a) P-FGM $\left(E_{M}<E_{C}\right)$ and E-FGM $\left(E_{M}>E_{C}\right)$ and (b) E-FGM $\left(E_{M}<E_{C}\right)$ and P-FGM $\left(E_{M}>E_{C}\right)$ beam. 


\subsubsection{Fully- and Partially- Clamped Cantilevered Beam Cases}

The fully- and partially- clamped cantilevered beam problems remain the same as the parameter specifications mentioned in section 5.2.1 and in Figures 5.15 and 5.19. For each scenario, the dynamic response for both fully- and partially- clamped cantilevered boundary conditions are depicted in: (i) Figures 5.37, 5.38 and 5.39; (ii) Figures 5.40, 5.41 and 5.42, and (iii) Figure 5.43 .

(i) Effect of change in average elastic moduli:

Figures 5.37 and 5.38 correspond to the transverse displacement response in the uncontrolledpassive and controlled-active-passive damping states, respectively. Figure 5.39 is the actuation voltage response with a gain of $K_{v}=350 \mathrm{~V} /(\mathrm{m} / \mathrm{s})$ applied to the composite beam. Within these figures, parts (a), (b), and (c) are the responses for $E_{\text {avg }}$ of $200 \mathrm{GPa}, 230$ GPa, and $260 \mathrm{GPa}$, respectively. Comparing the fully- and partially- clamped cantilevered responses, it is evident that the partially-clamped response has greater transverse displacement than the fully-clamped response due to its flexibility, since only the host beam is constrained. Consistently, the Q2DT E-FGM has the lowest peak-to-peak amplitude, followed by the homogeneous, and then by the Q2DT P-FGM response. With increasing $E_{\text {avg }}$, the Q2DT E-FGM decreases in transverse displacement, resulting in better damping capabilities than the Q2DT P-FGM and homogeneous responses. Furthermore, the frequency of the response increases with increasing $E_{\text {avg }}$.

For both passive and active-passive damping, Figures 5.37 and 5.38, as $E_{\text {avg }}$ increases, the Q2DT P-FGM gradually increases in peak-to-peak amplitude and becomes more out of phase with the Q2DT E-FGM and homogeneous responses. For the fully-clamped cantilevered case, as $E_{\text {avg }}$ increases, the Q2DT E-FGM gradually becomes more in-phase with the homogeneous response. For the fully-clamped case, however, as $E_{\text {avg }}$ increases, the partially-clamped Q2DT E-FGM gradually becomes more out of phase with the homogeneous response. The actuator voltage responses in Figure 5.39 are proportional to the rate of deflection responses in Figure 5.38 . 


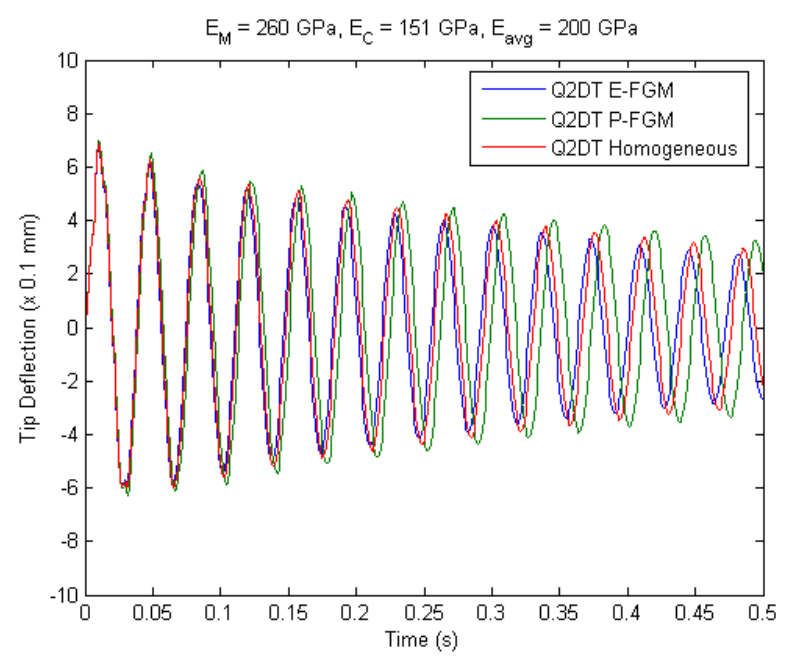

$(1 a)$

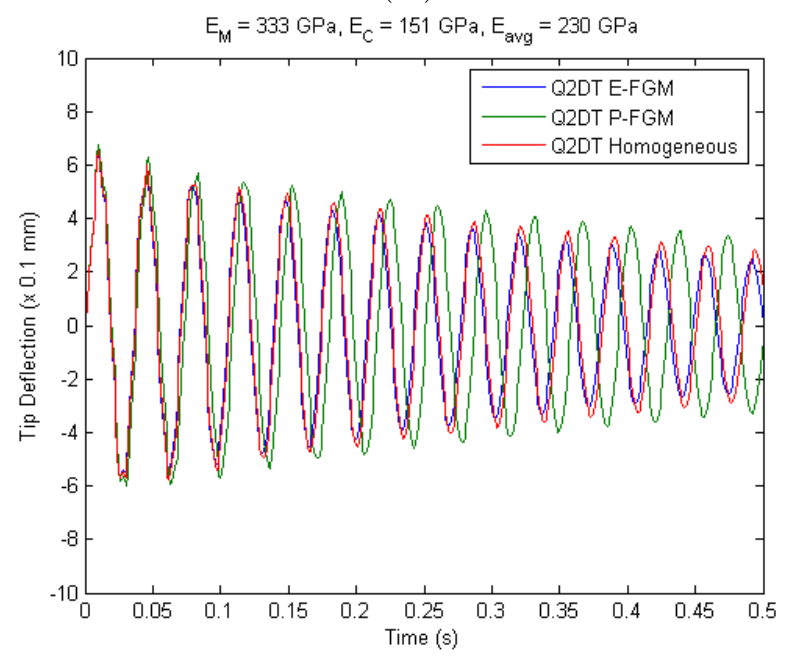

$(1 b)$

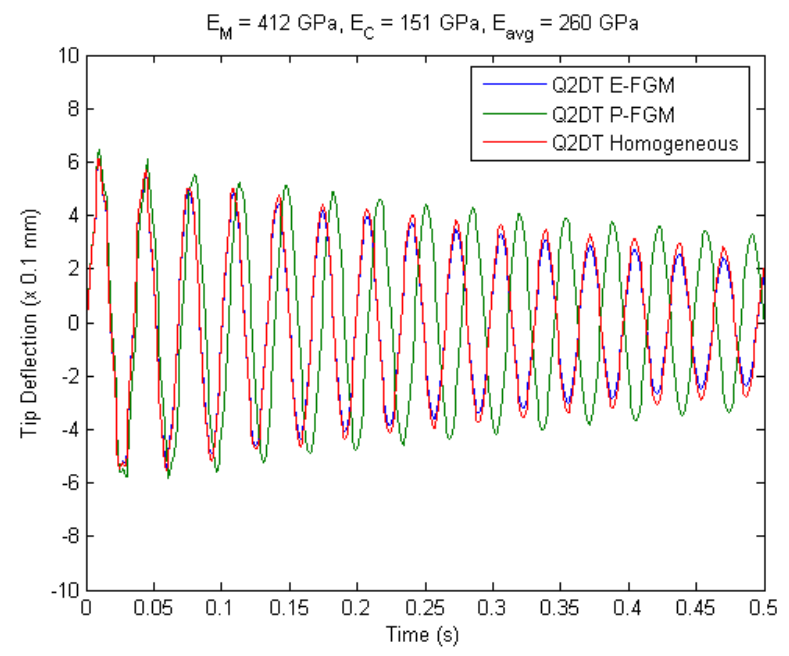

$(1 c)$

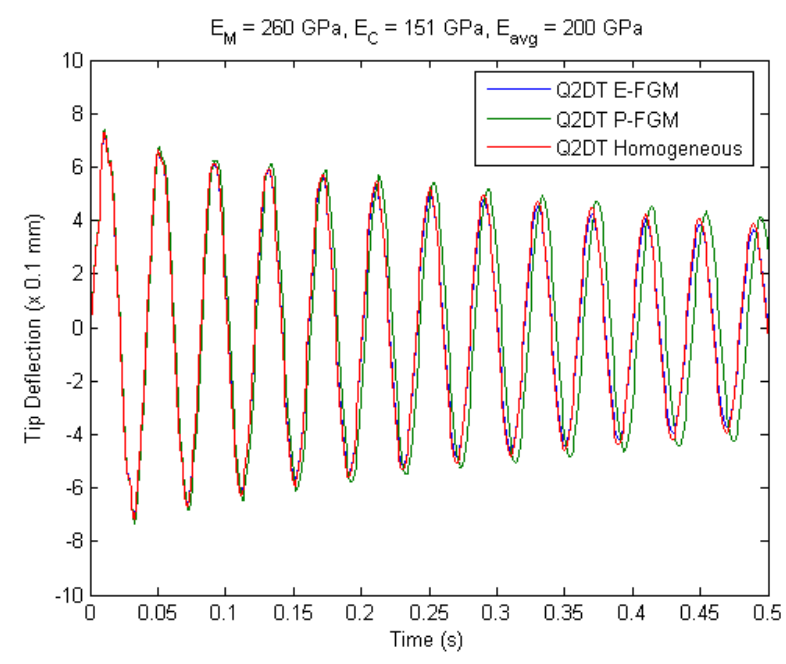

$(2 a)$

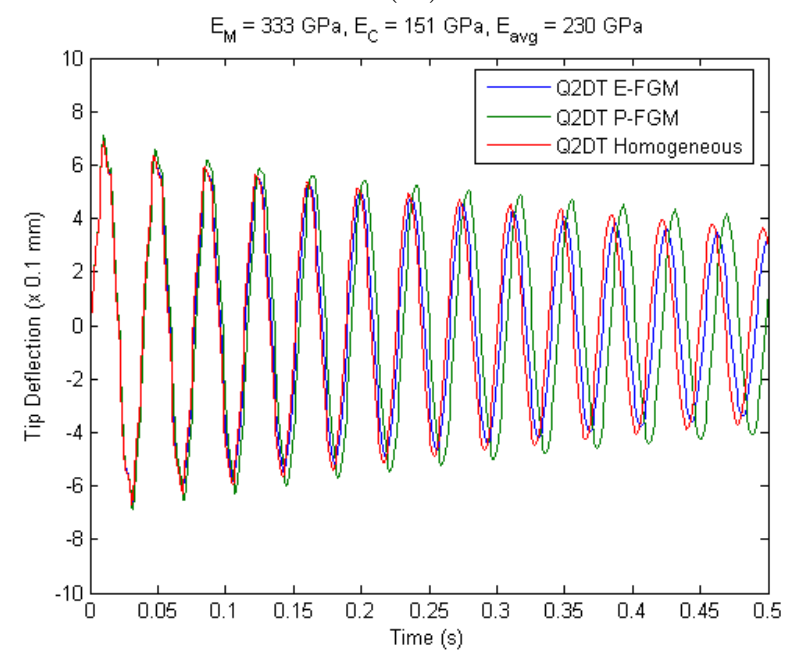

$(2 b)$

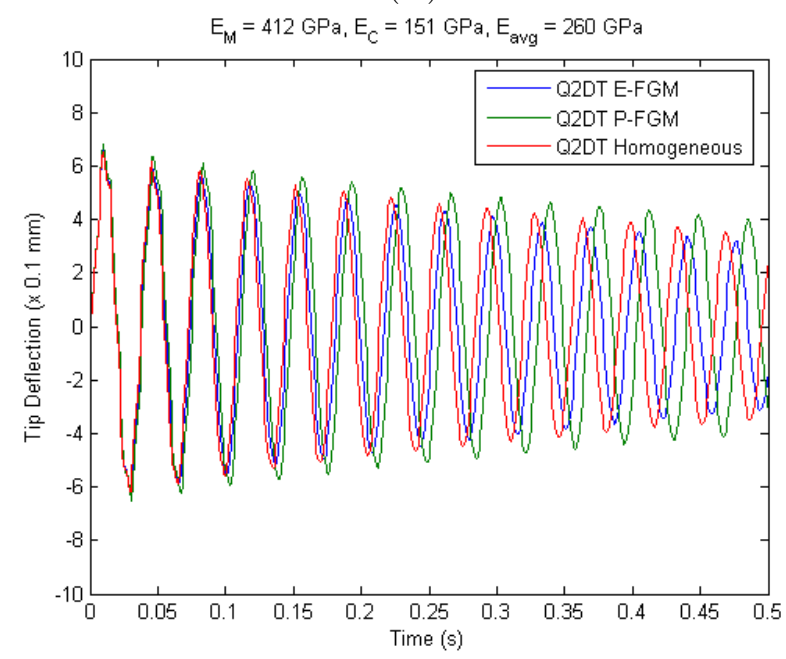

$(2 c)$

Figure 5.37: Effect of change in average elastic modulus on the tip transverse displacement for uncontrolled-passive damping of $(1 \mathrm{a}),(1 \mathrm{~b}),(1 \mathrm{c})$ fully-clamped and $(2 \mathrm{a}),(2 \mathrm{~b}),(2 \mathrm{c})$ partiallyclamped cantilevered beam. 


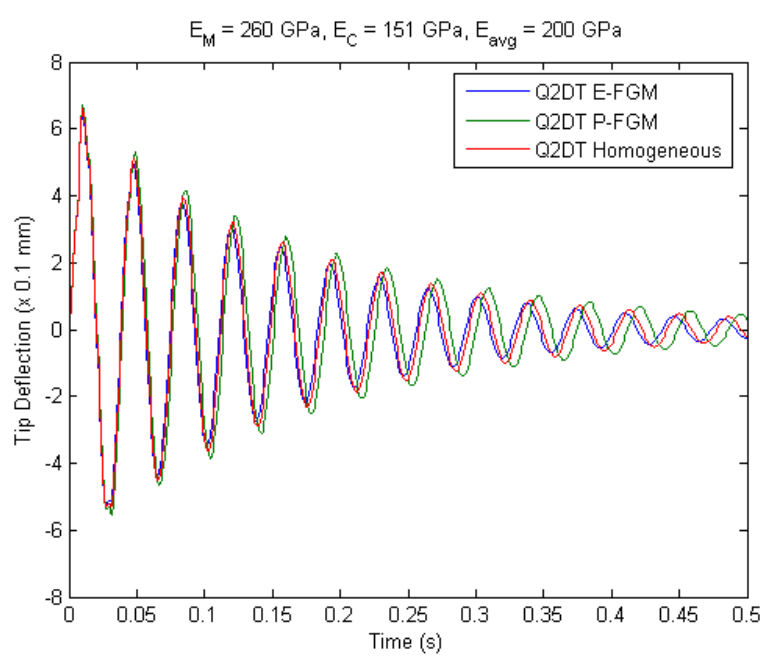

(1a)

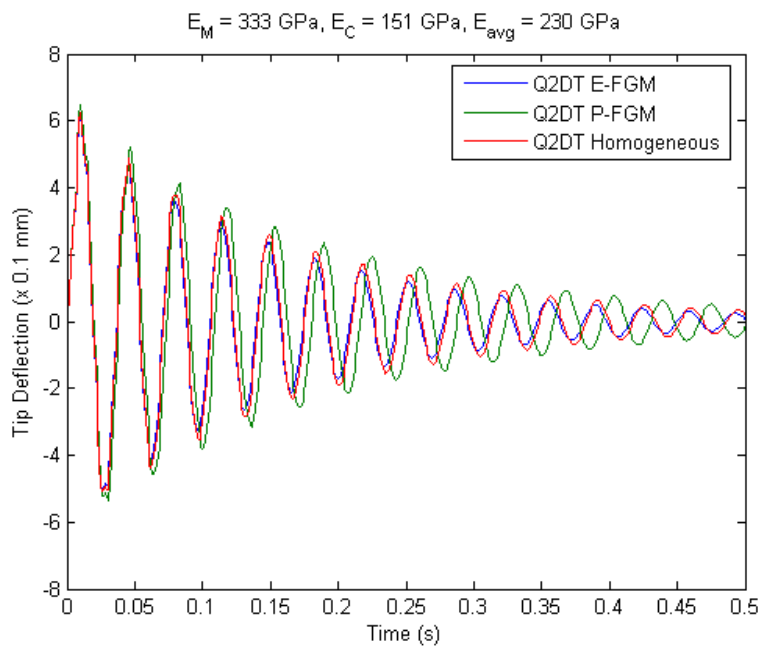

$(1 b)$

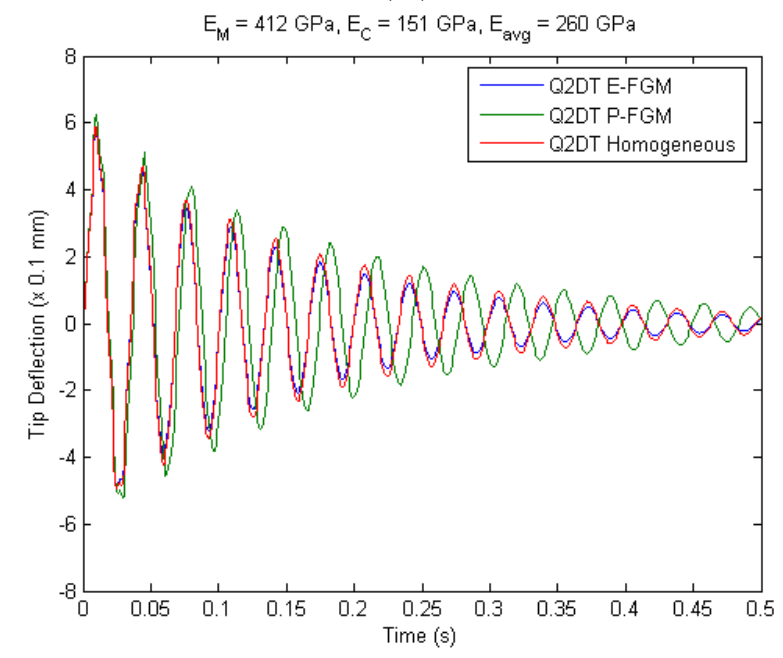

$(1 c)$

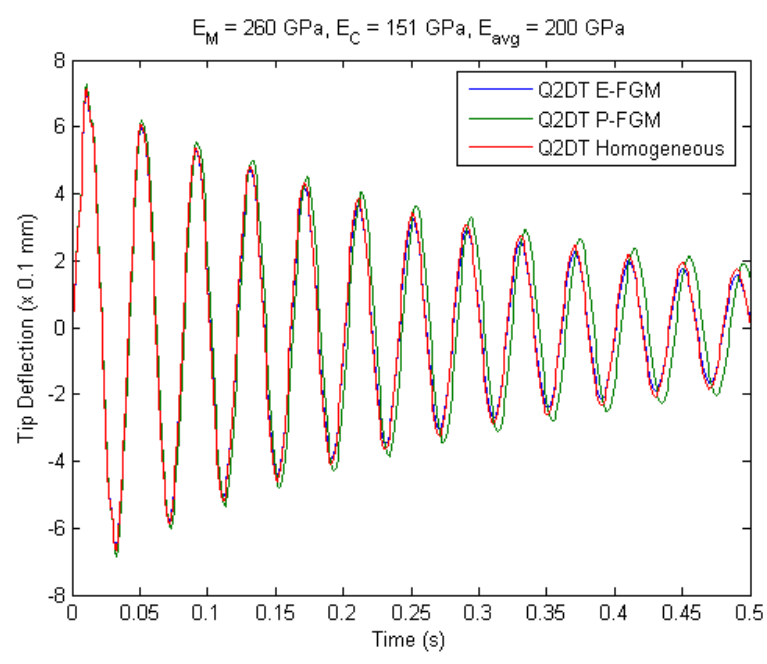

$(2 a)$

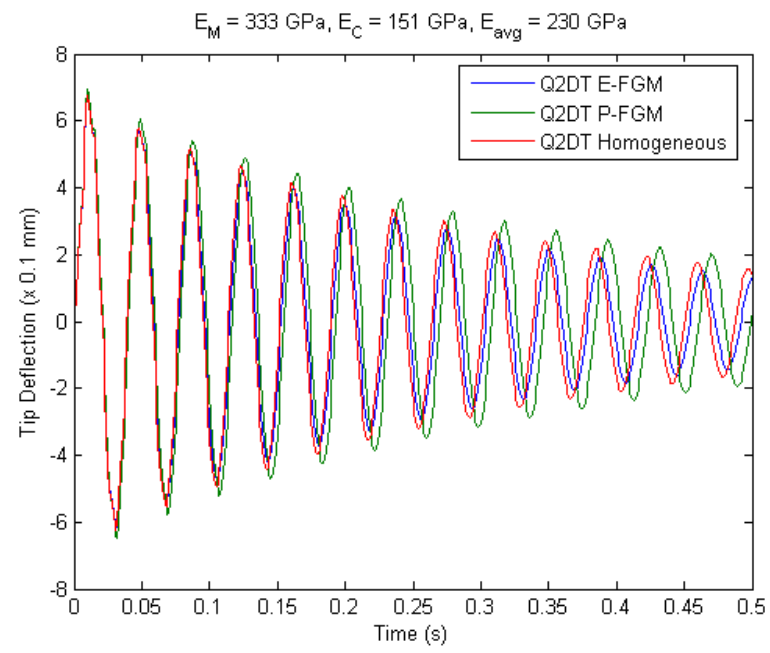

$(2 b)$

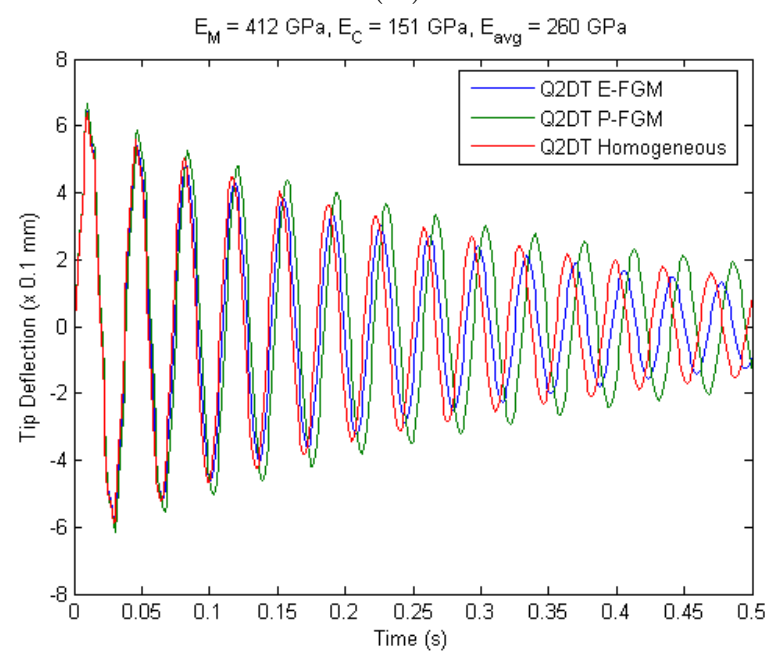

$(2 c)$

Figure 5.38: Effect of change in average elastic modulus on the tip transverse displacement for controlled-active-passive damping of $(1 \mathrm{a}),(1 \mathrm{~b}),(1 \mathrm{c})$ fully-clamped and $(2 \mathrm{a}),(2 \mathrm{~b}),(2 \mathrm{c})$ partiallyclamped cantilevered beam. 


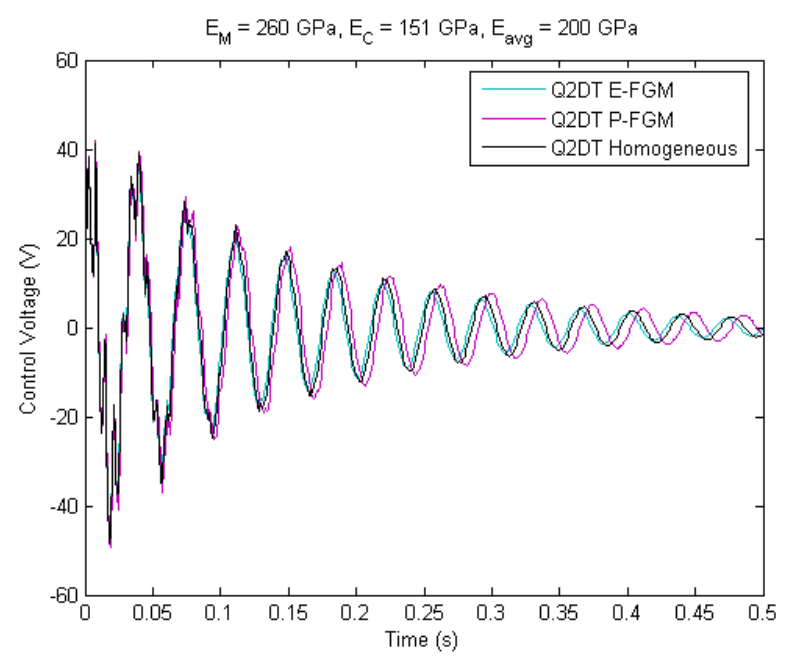

(1a)

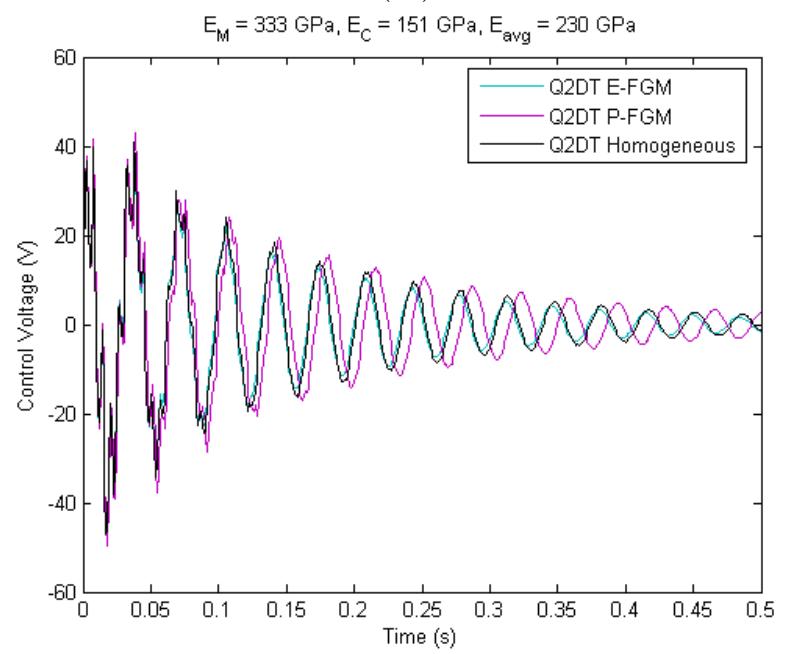

$(1 b)$

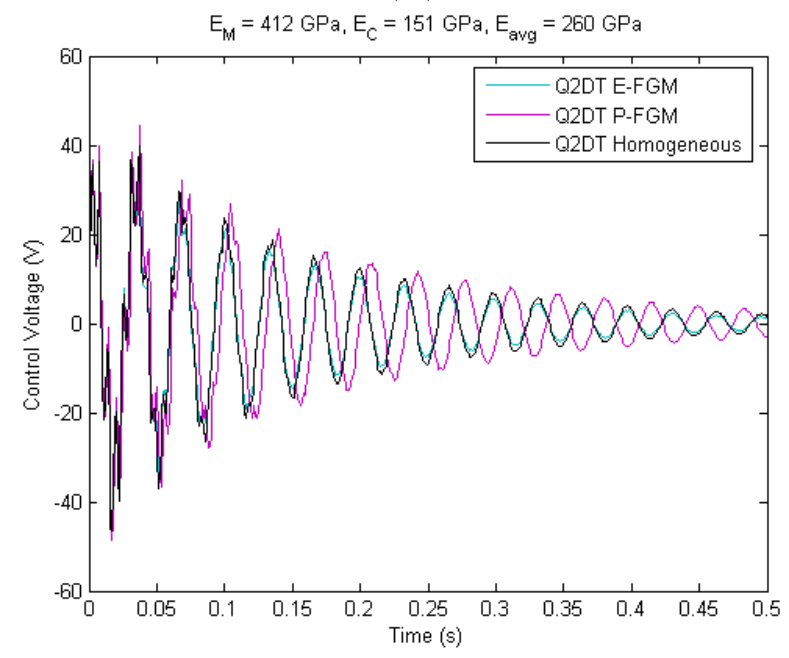

$(1 c)$

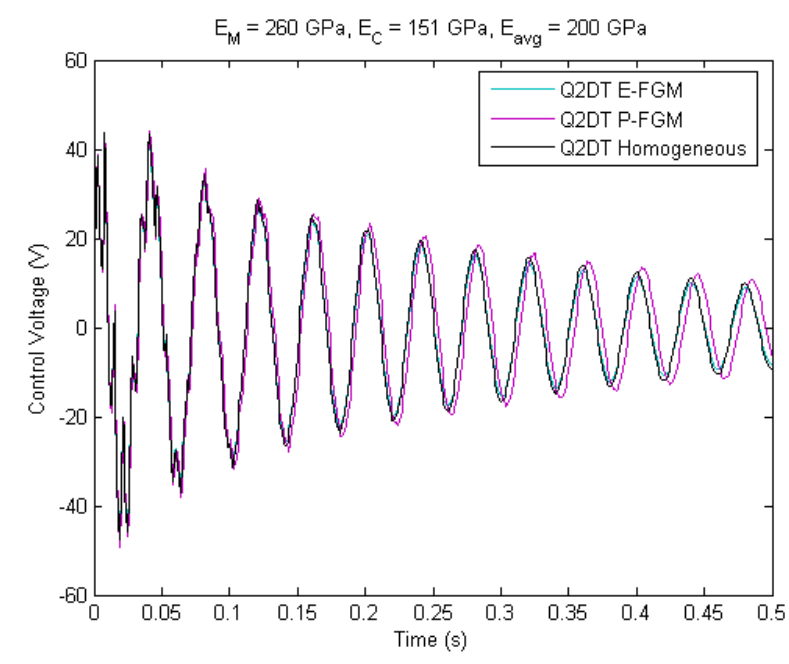

$(2 a)$

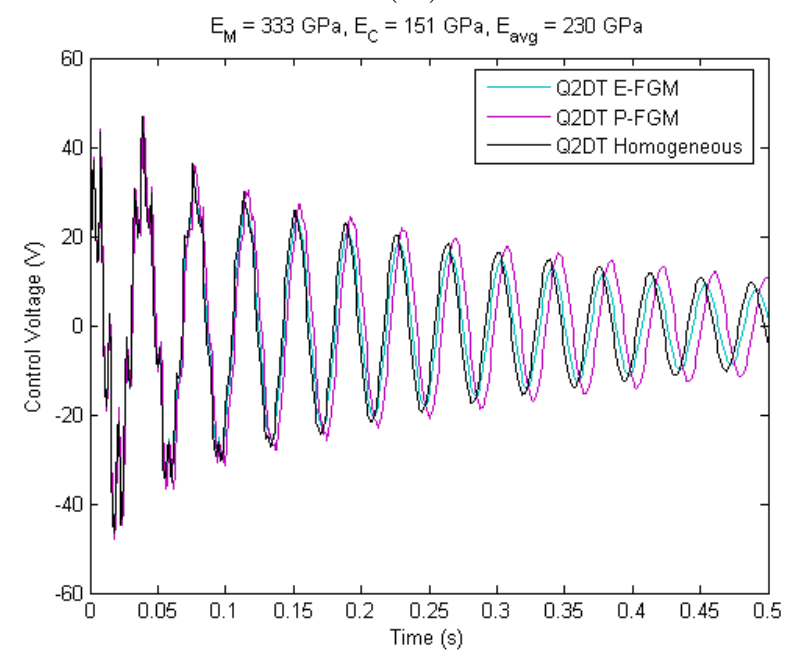

$(2 b)$

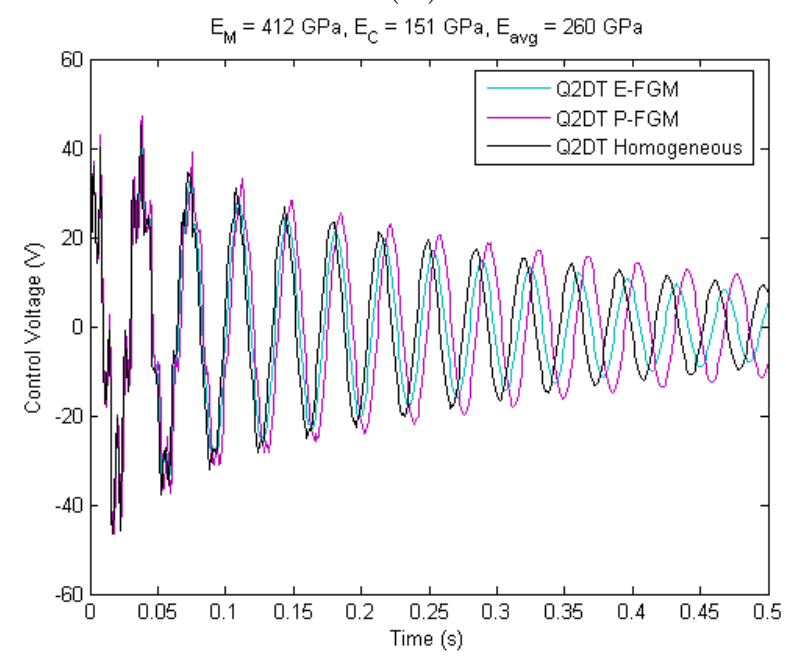

$(2 c)$

Figure 5.39: Effect of change in average elastic modulus on the actuation voltage for controlled-active-passive damping of $(1 \mathrm{a}),(1 \mathrm{~b}),(1 \mathrm{c})$ fully-clamped and $(2 \mathrm{a}),(2 \mathrm{~b}),(2 \mathrm{c})$ partiallyclamped cantilevered beam. 
(ii) Effect of varying constituents moduli for fixed average elastic moduli:

Figures 5.40 and 5.41 represent the uncontrolled-passive and controlled-active-passive tip displacement responses with $E_{\text {avg }}$ of $200 \mathrm{GPa}$, respectively. Both tip displacements follow the same trend and observations. Figure 5.42 is the actuation voltage response of the controlled-active-passive damping, which is proportional to the displacement response. For each part (a), (b), and (c), the $\Delta E$ 's are $109 \mathrm{GPa}, 252 \mathrm{GPa}$, and $366 \mathrm{GPa}$, respectively (as shown in Table 5.9). The increase in $\Delta E$ could be advantageous or disadvantageous depending on the chosen FG function. For example, as $\Delta E$ increases, the Q2DT P-FGM response increases in peak-to-peak amplitude of vibration, while the Q2DT E-FGM response decreases in amplitude of vibration. 


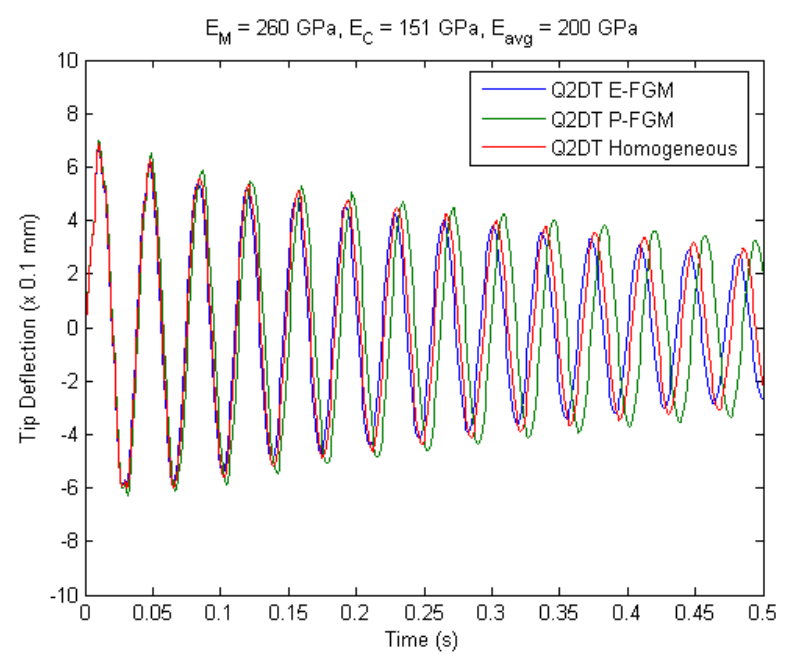

(1a)

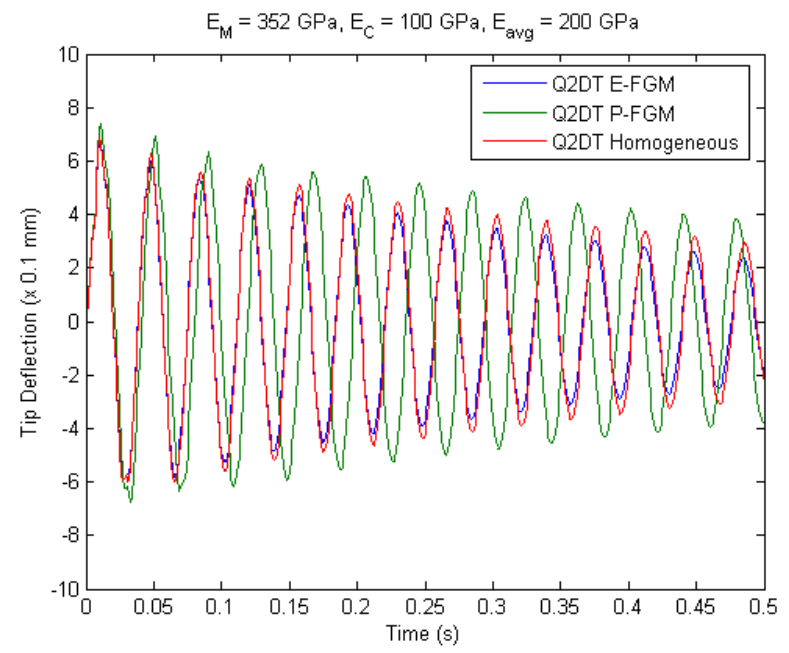

$(1 b)$

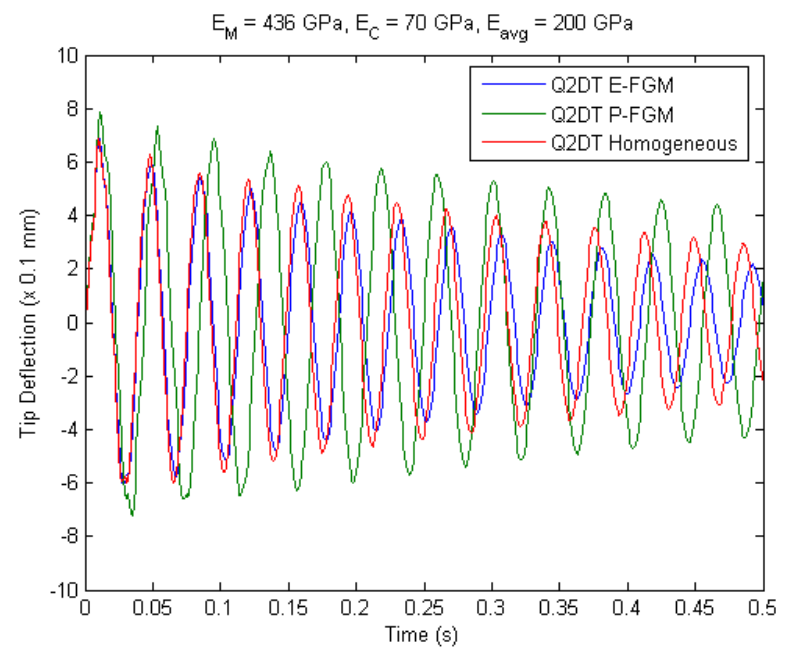

$(1 c)$

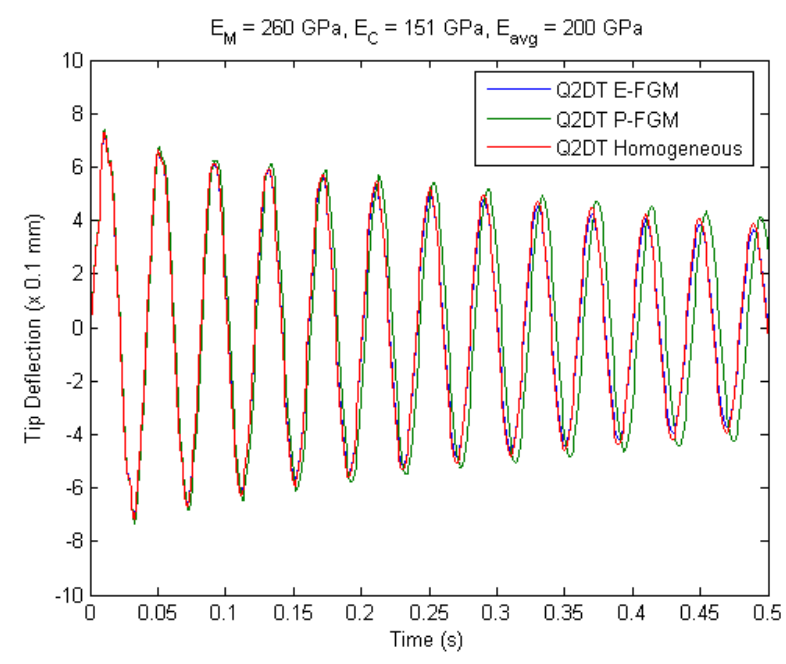

$(2 a)$

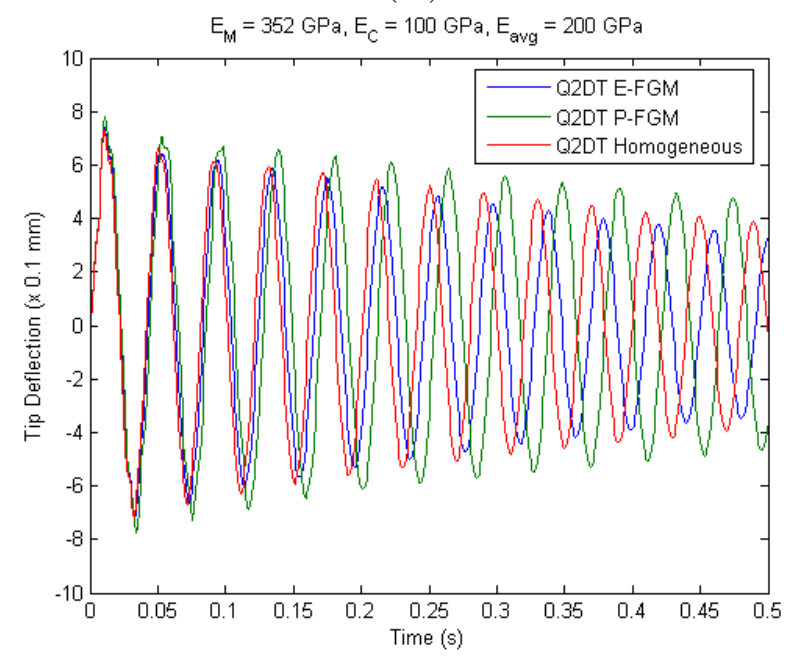

$(2 b)$

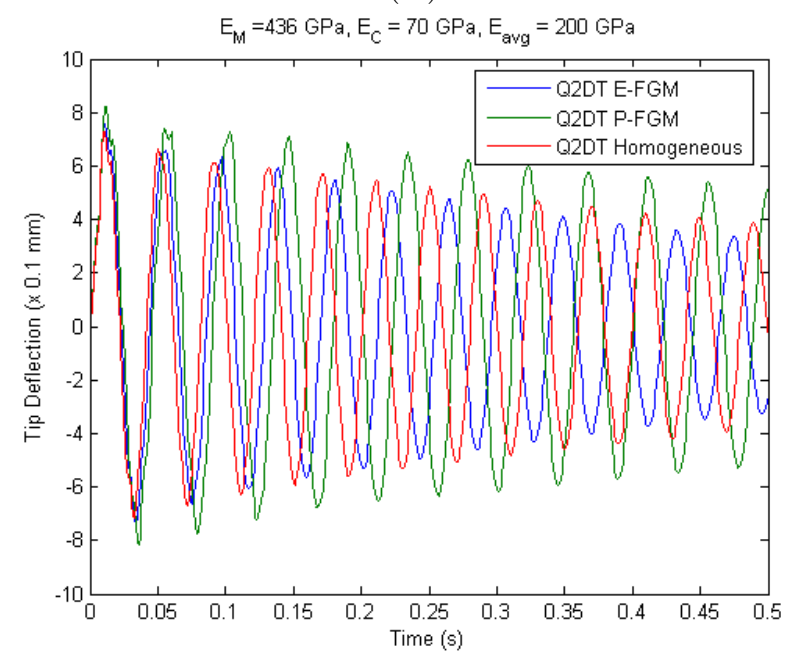

$(2 c)$

Figure 5.40: Effect of varying constituents moduli for fixed average elastic modulus on the tip transverse displacement for uncontrolled-passive damping of (1a),(1b),(1c) fully-clamped and $(2 \mathrm{a}),(2 \mathrm{~b}),(2 \mathrm{c})$ partially-clamped cantilevered beam. 


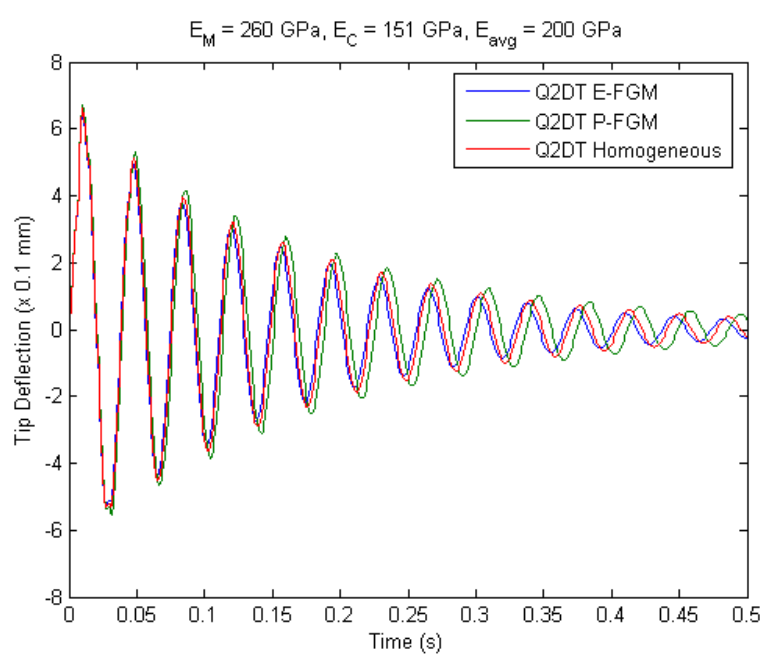

(1a)

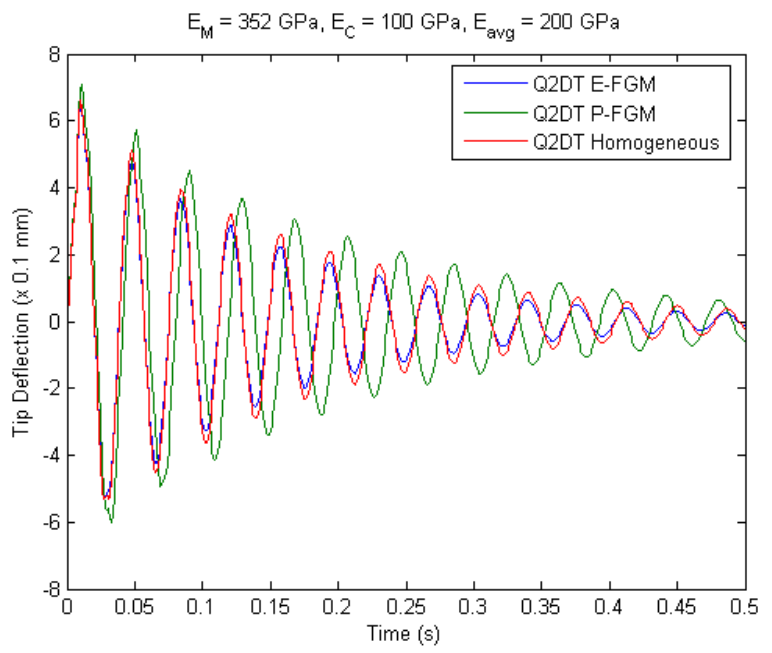

$(1 b)$

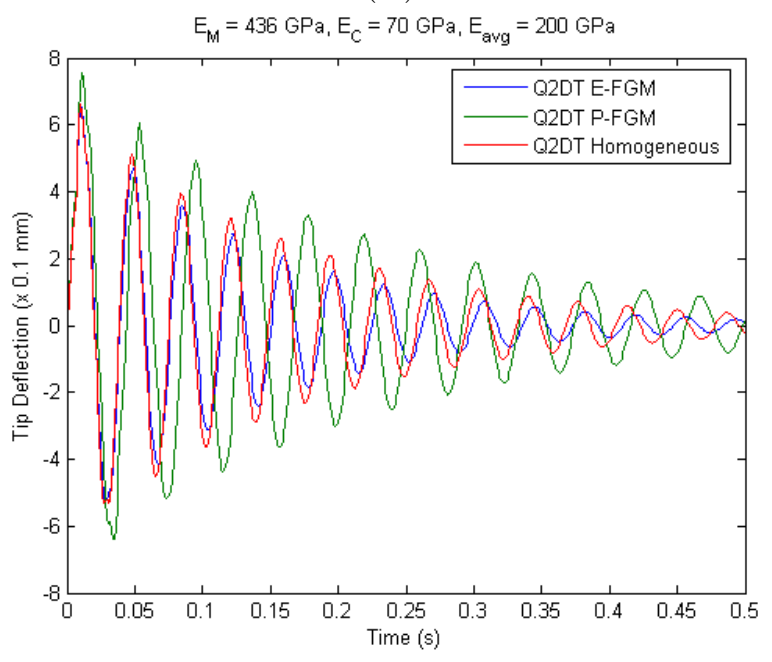

$(1 c)$

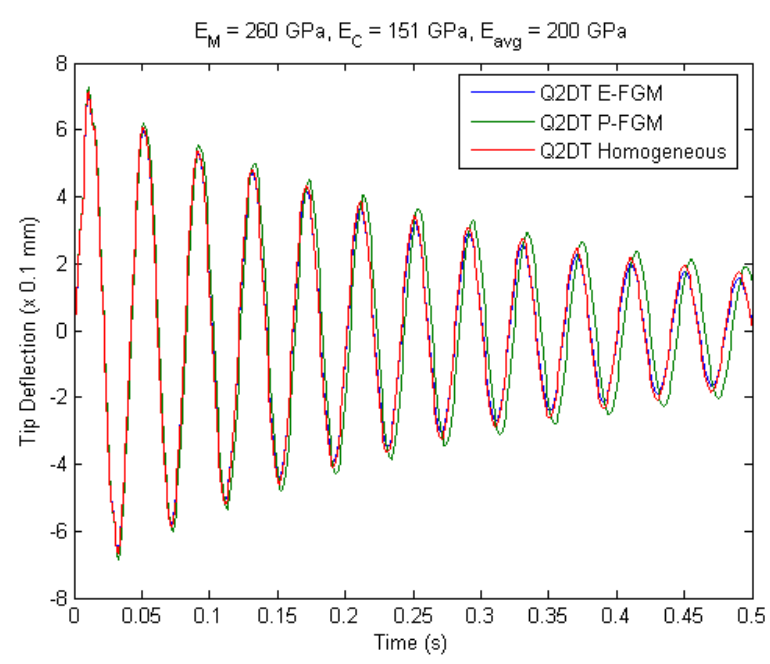

$(2 a)$

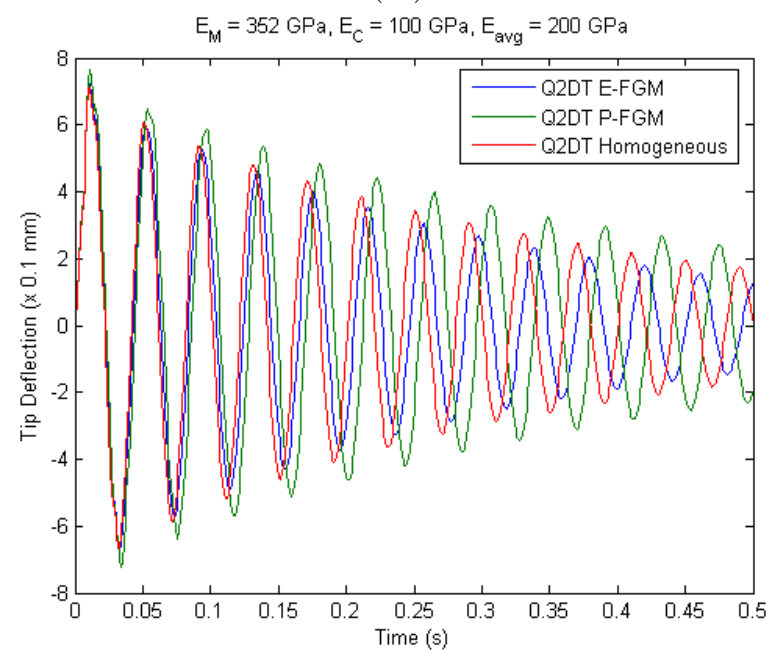

$(2 b)$

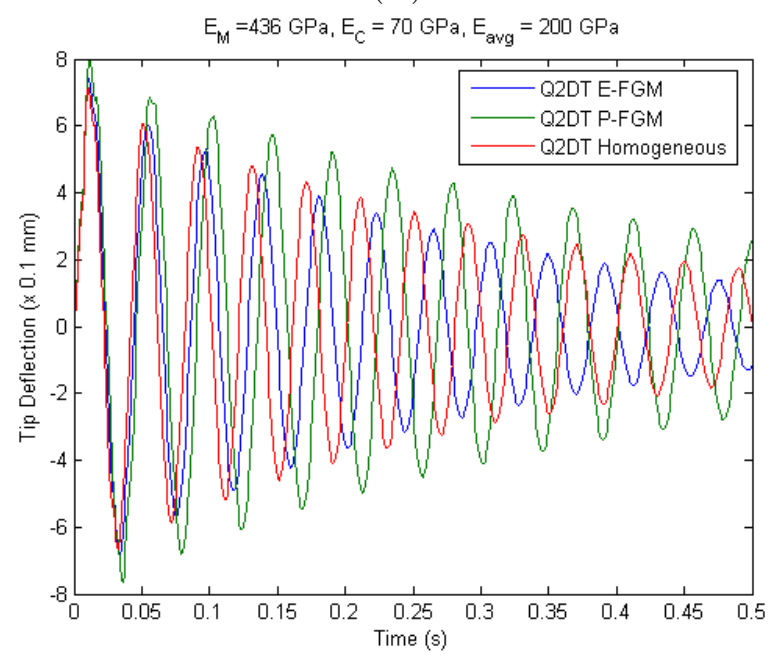

$(2 c)$

Figure 5.41: Effect of varying constituents moduli for fixed average elastic modulus on the tip transverse displacement for controlled-active-passive damping of (1a),(1b),(1c) fully-clamped and $(2 \mathrm{a}),(2 \mathrm{~b}),(2 \mathrm{c})$ partially-clamped cantilevered beam. 


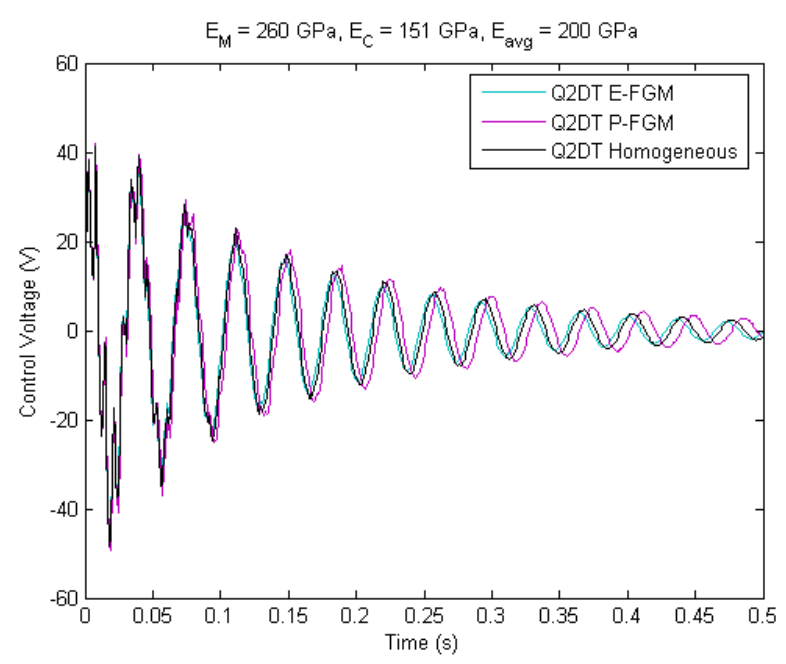

(1a)

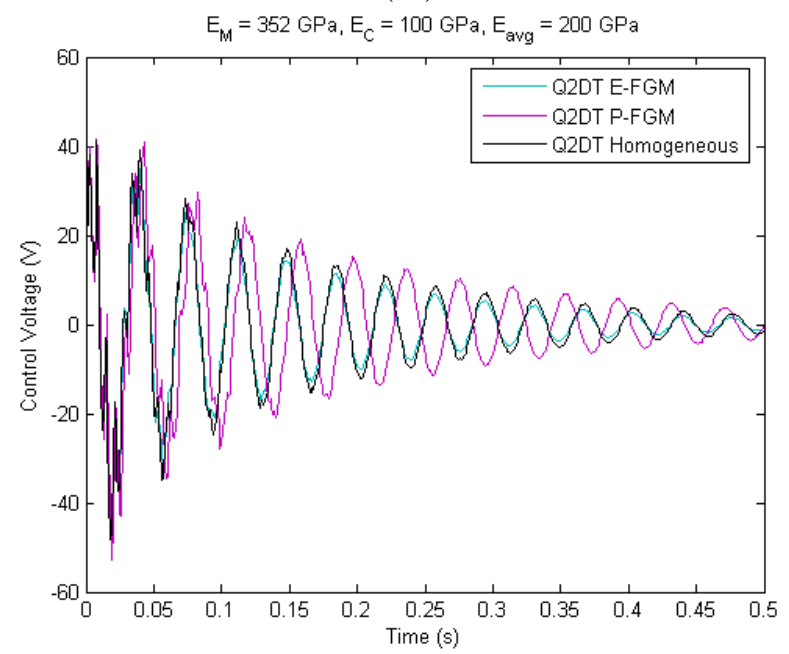

$(1 b)$

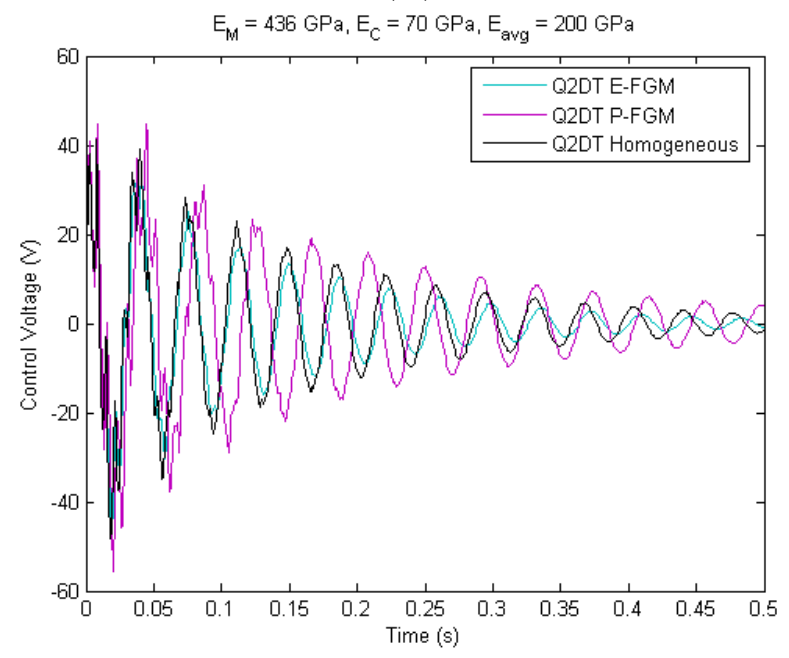

$(1 c)$

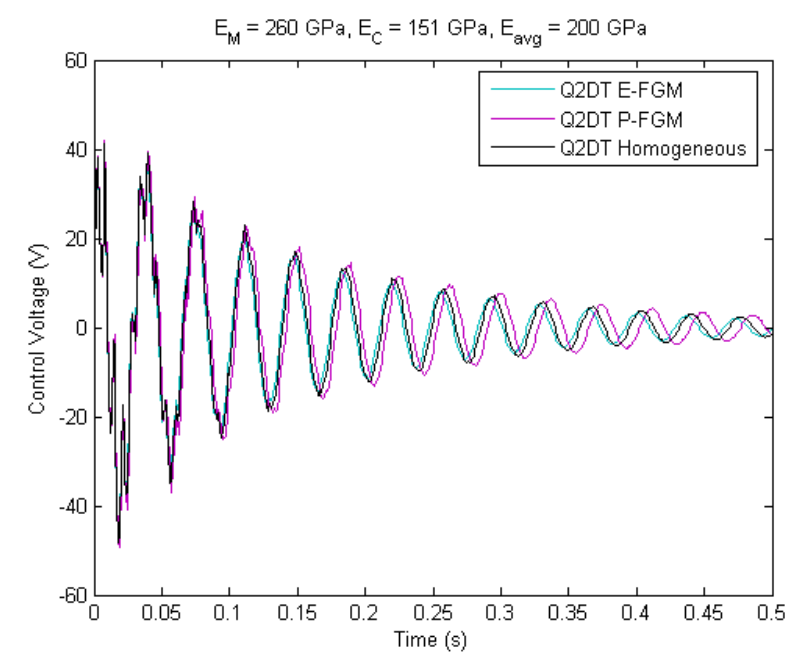

$(2 a)$

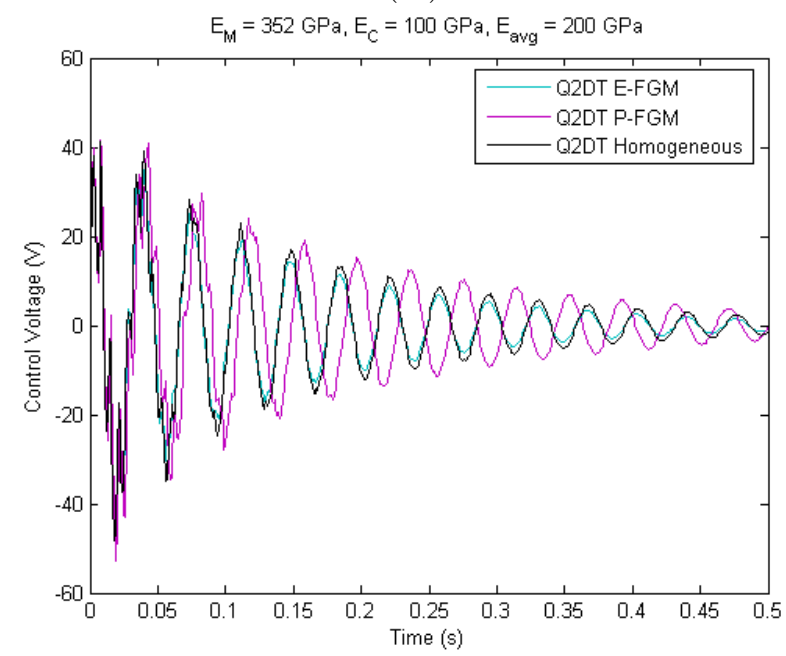

$(2 b)$

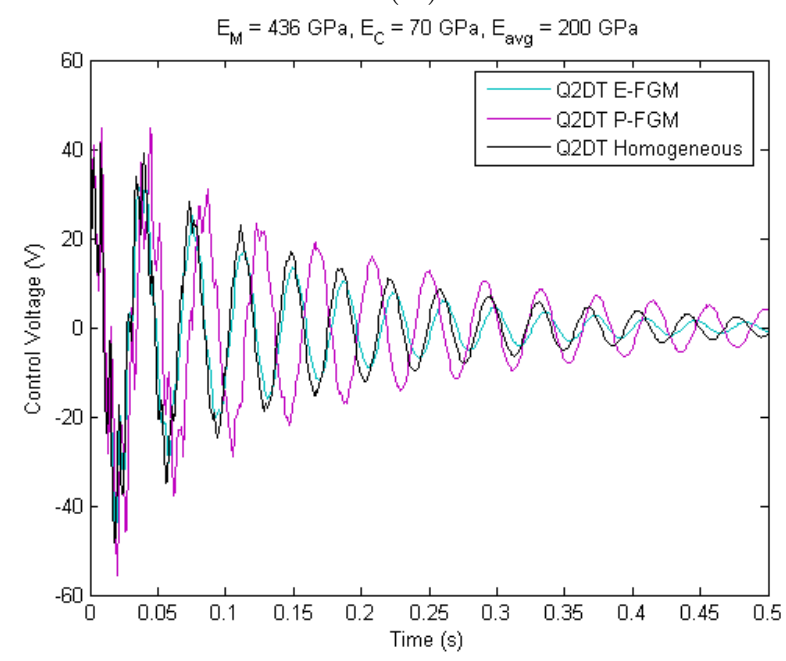

$(2 c)$

Figure 5.42: Effect of varying constituents moduli for fixed average elastic modulus on the actuation voltage for controlled-active-passive damping of (1a),(1b),(1c) fully-clamped and (2a), (2b),(2c) partially-clamped cantilevered beam. 
(iii) Effect of exchange in the metal to ceramic and ceramic to metal elastic moduli:

Figure 5.43 is the uncontrolled-passive damping response for the exchange of metal to ceramic and ceramic to metal elastic moduli. Part (a) for both the fully- and partiallyclamped cantilevered responses show that at a low $\Delta E$ of $109 \mathrm{GPa}$, the responses match almost exactly for both Q2DT E-FGM and P-FGM. However, in part (b), only the Q2DT E-FGM $\left(E_{M}>E_{C}\right)$ and Q2DT P-FGM $\left(E_{M}<E_{C}\right)$ match when $\Delta E$ is increased to 252 GPa. The Q2DT P-FGM $\left(E_{M}>E_{C}\right)$ and Q2DT E-FGM $\left(E_{M}<E_{C}\right)$ result in the same amplitude of vibration, but an apparent phase shift from each other. Furthermore, the phase shift shown in part (b) becomes larger in part (c). Also, in part (c) the responses that used to match do not match anymore, especially for the partially-clamped case, where there is a slight phase shift. As $\Delta E$ increases, the Q2DT P-FGM $\left(E_{M}>E_{C}\right)$ and Q2DT E-FGM $\left(E_{M}<E_{C}\right)$ responses continue to increase in amplitude in comparison to the homogeneous response. On the other hand, as $\Delta E$ increases, the Q2DT E-FGM $\left(E_{M}>E_{C}\right)$ and Q2DT P-FGM $\left(E_{M}<E_{C}\right)$ responses decrease in amplitude in comparison to the homogeneous response. In order to obtain the least amount of vibration, either the Q2DT E-FGM $\left(E_{M}>E_{C}\right)$ or Q2DT P-FGM $\left(E_{M}<E_{C}\right)$ responses should be used. For example, should the exponential function be used to represent a FG beam, the top elastic modulus must be greater than the bottom. For a power-law function FG beam, the top elastic modulus should be less than the bottom. 


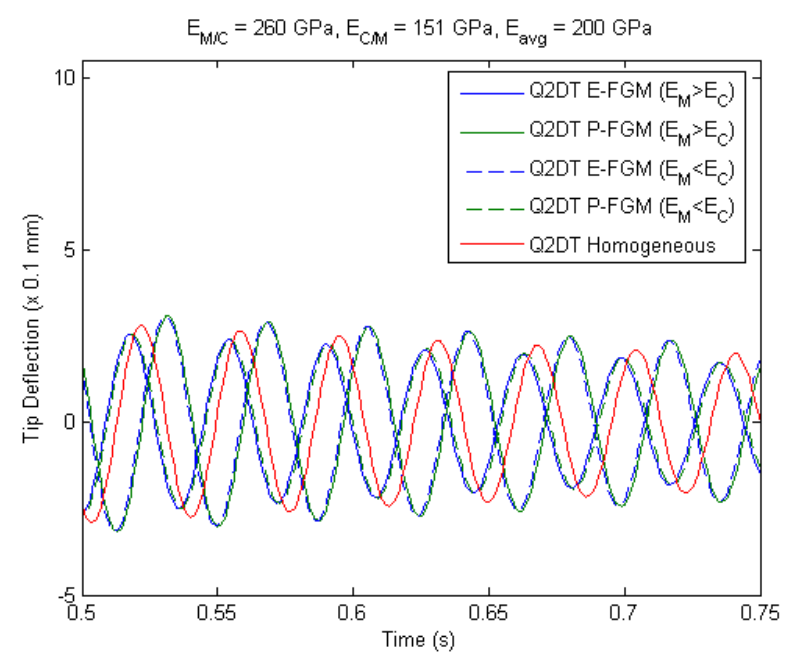

$(1 a)$

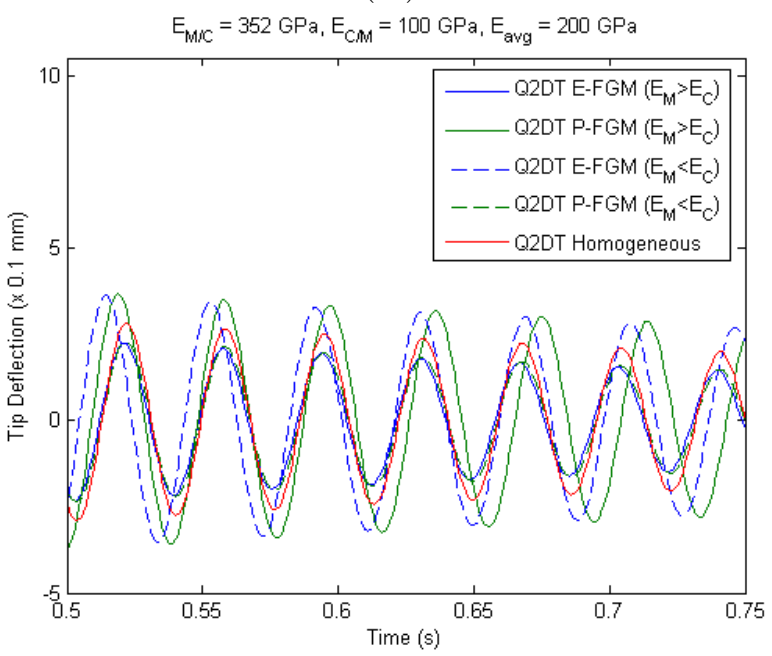

$(1 b)$

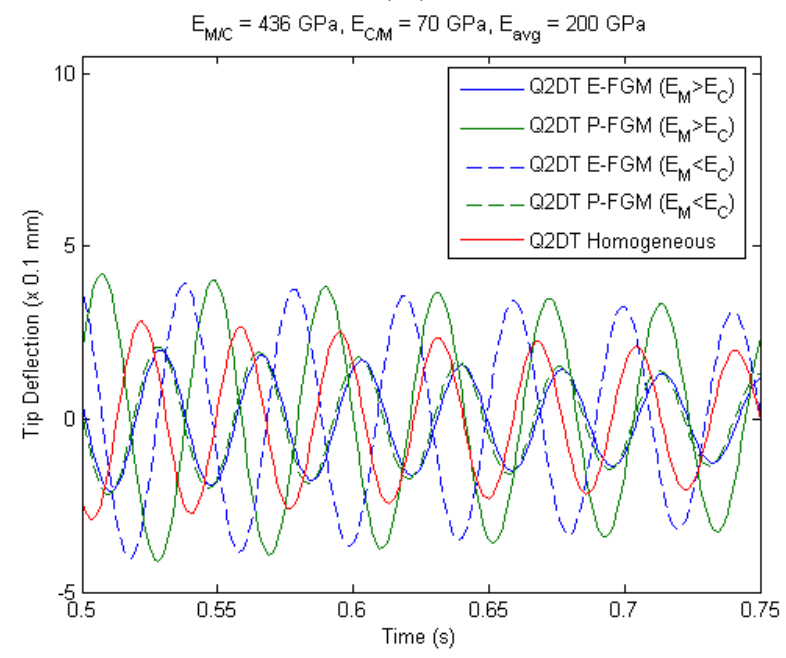

$(1 c)$

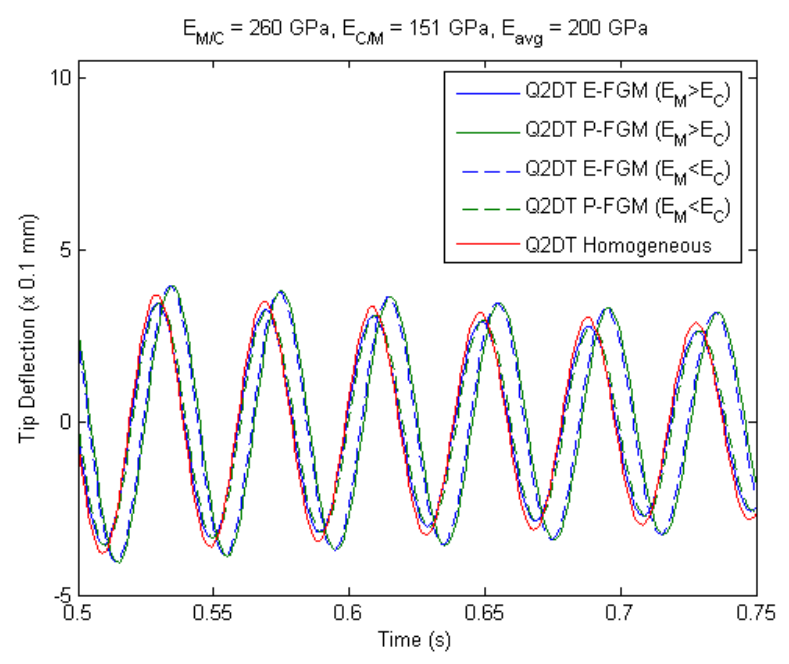

$(2 a)$

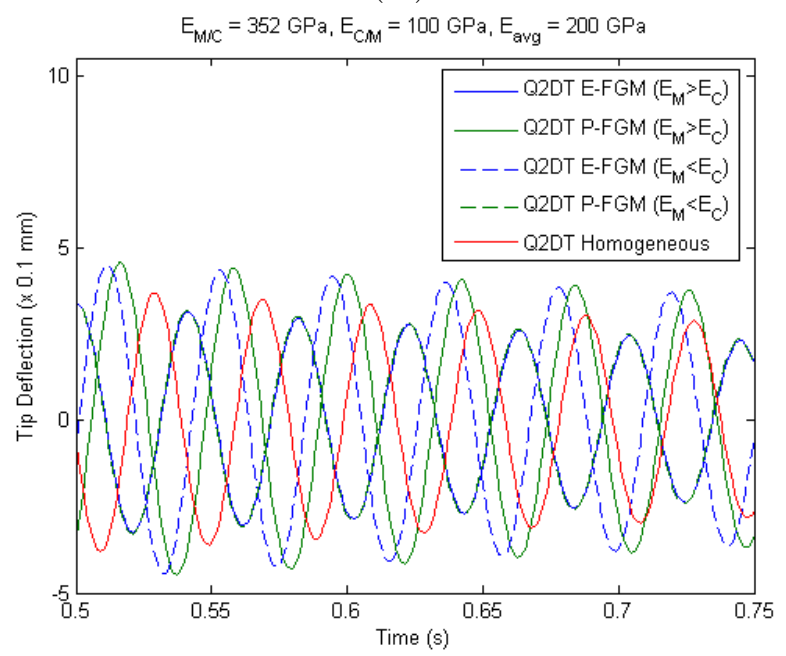

$(2 b)$

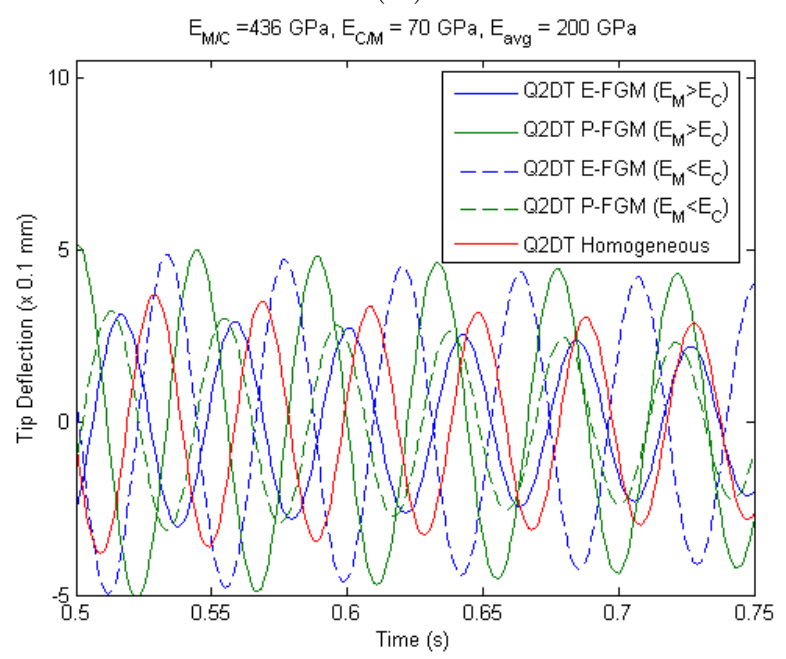

$(2 c)$

Figure 5.43: Effect of exchange in metal and ceramic elastic moduli on the tip transverse displacement for uncontrolled-passive damping of $(1 \mathrm{a}),(1 \mathrm{~b}),(1 \mathrm{c})$ fully-clamped and $(2 \mathrm{a}),(2 \mathrm{~b}),(2 \mathrm{c})$ partially-clamped cantilevered beam. 


\subsubsection{Partially Clamped-clamped Beam Case}

A clamped-clamped ACLD beam that is constrained only at the host beam is shown in Figure 5.26 in Section 5.2.1. The same parameters are used in this section. Also, the same response sequence is repeated as in the previous case, for the three scenarios, which includes the response due to passive damping, active-passive damping, and control voltage.

(i) Effect of change in average elastic moduli:

Figures 5.44, 5.45 and 5.46 depict the passive damped, active-passive damped, and control voltage responses, respectively. As the average elastic modulus $E_{\text {avg }}$ increases, the same pattern is observed as the cantilevered cases, wherein, the Q2DT P-FGM response increases, while the Q2DT E-FGM response decreases in its peak-to-peak amplitudes of vibration relative to the homogeneous response. The frequency of vibration also increases as the $E_{\text {avg }}$ increases. A careful observation of the Q2DT E-FGM responses shows improved damping with higher $E_{a v g}$. These patterns are the same for all three responses (passive, active-passive, and control voltage). 


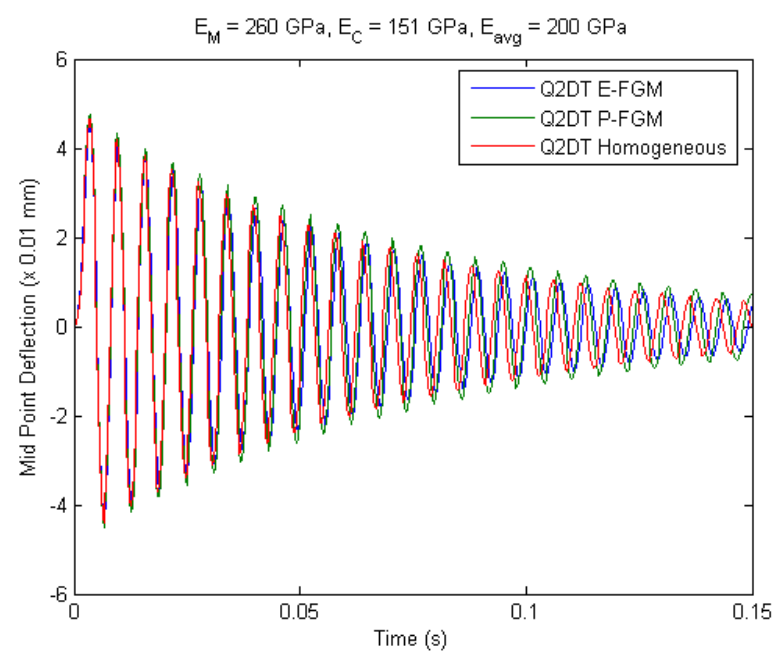

(a)

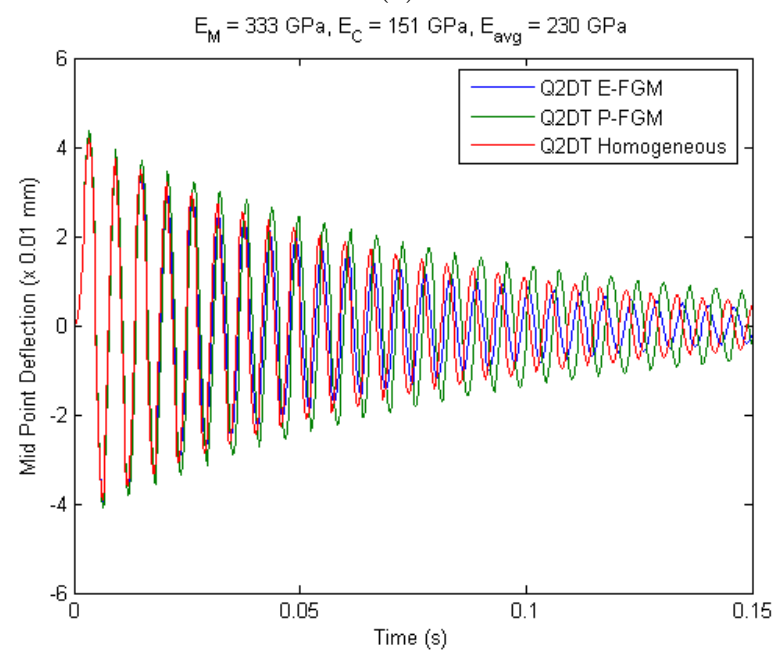

(b)

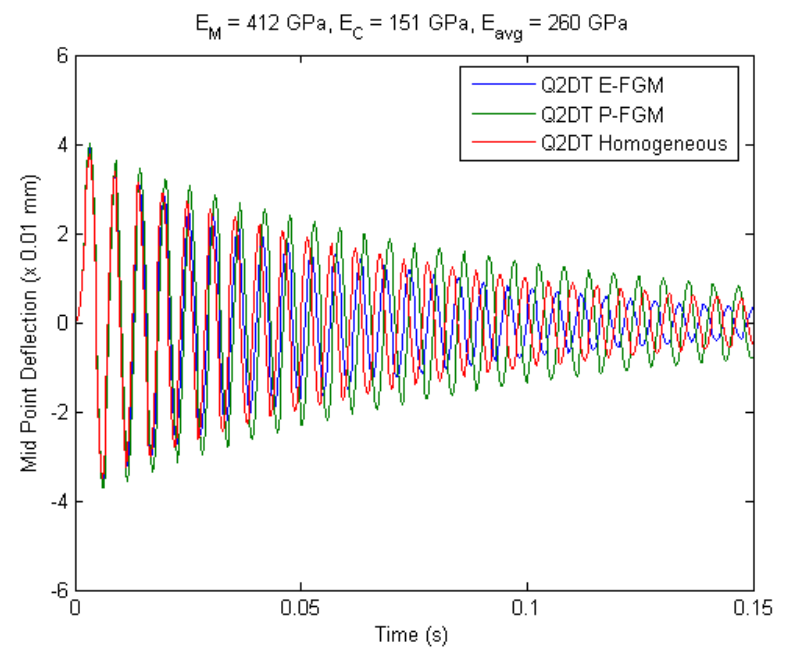

(c)

Figure 5.44: Effect of change in average elastic modulus on the mid-point transverse displacement for uncontrolled-passive damping of partially clamped-clamped beam. (a),(b),(c) $E_{a v g}=200 \mathrm{GPa}, 230 \mathrm{GPa}$, and $260 \mathrm{GPa}$. 


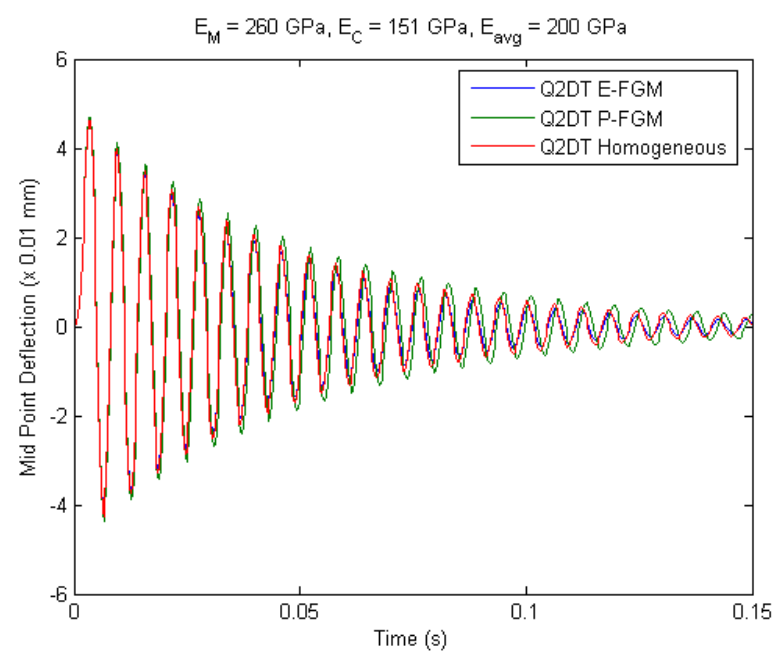

(a)

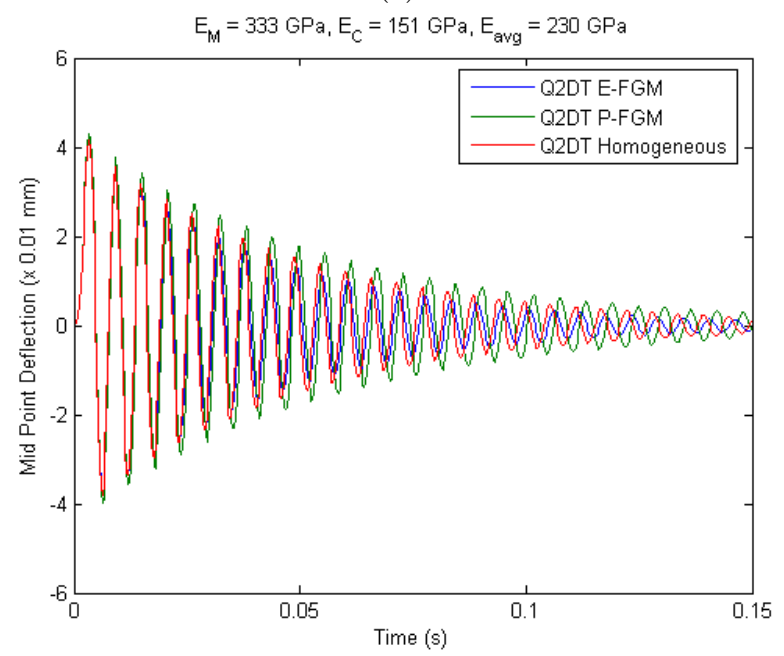

$(b)$

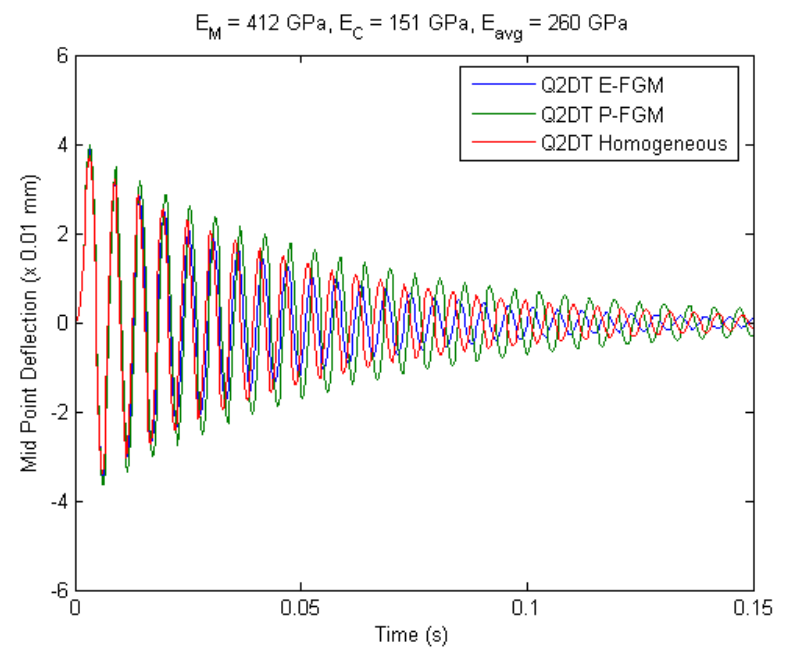

(c)

Figure 5.45: Effect of change in average elastic modulus on the mid-point transverse displacement for controlled-active-passive damping of partially clamped-clamped beam. (a),(b),(c) $E_{a v g}=200 \mathrm{GPa}, 230 \mathrm{GPa}$, and $260 \mathrm{GPa}$. 


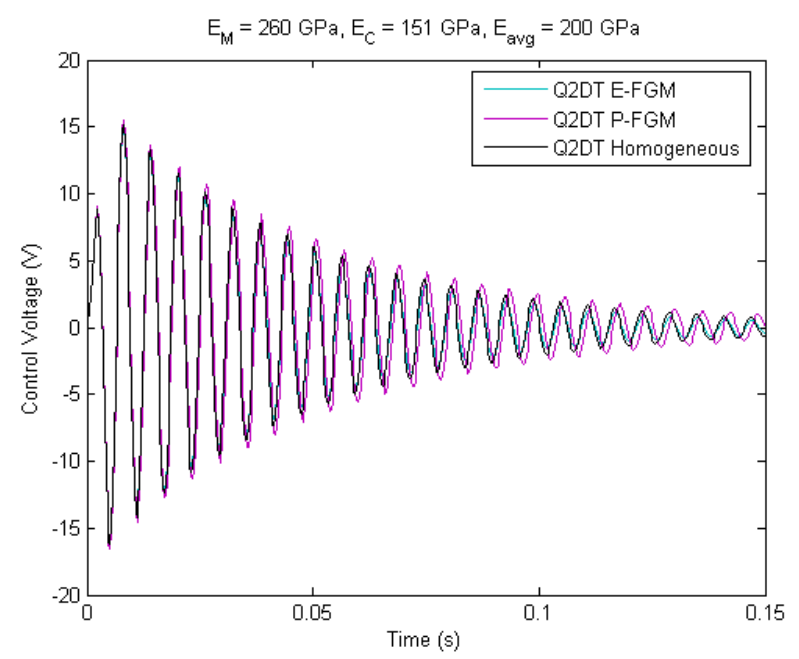

(a)

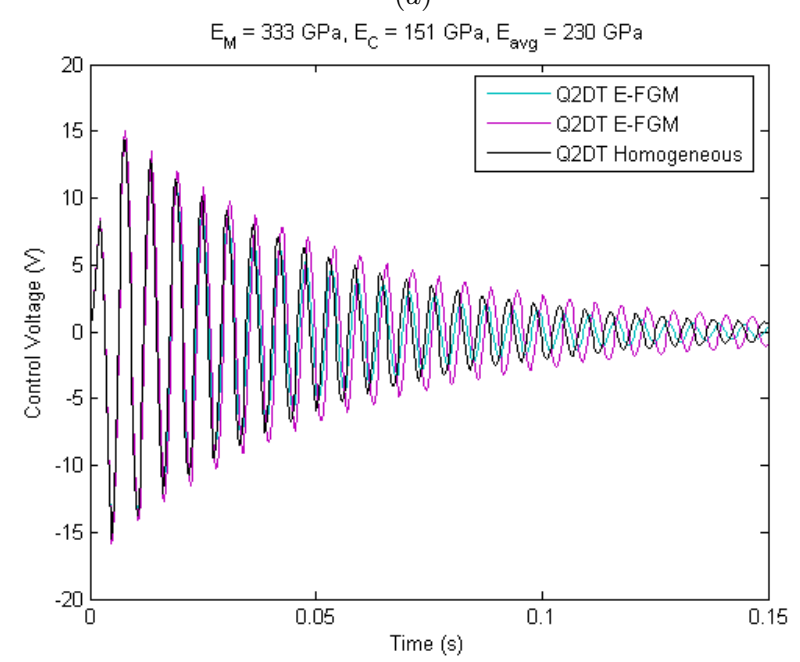

$(b)$

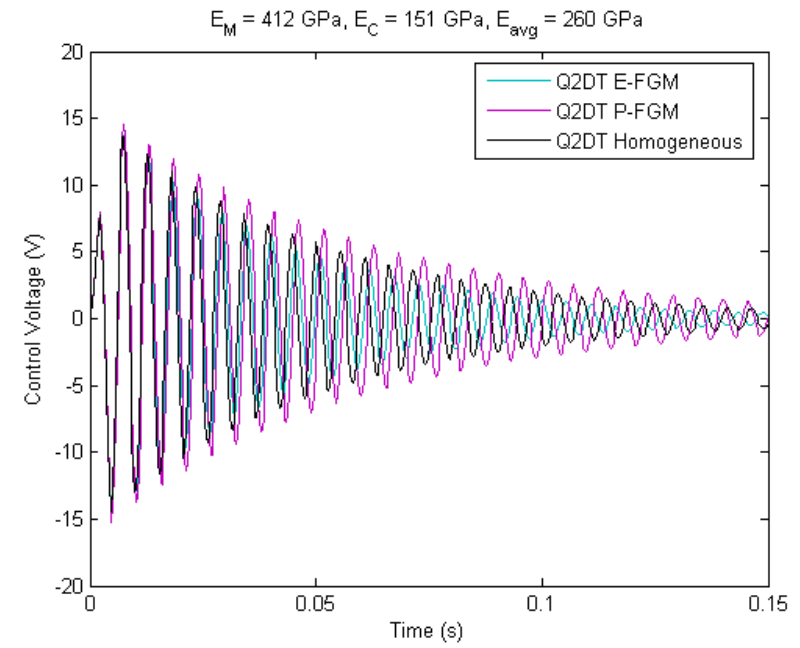

(c)

Figure 5.46: Effect of change in average elastic modulus on the actuation voltage for controlled-active-passive damping of partially clamped-clamped beam. (a),(b),(c) $E_{a v g}=200$ GPa, $230 \mathrm{GPa}$, and $260 \mathrm{GPa}$. 
(ii) Effect of varying constituents moduli for fixed average elastic modulus:

The passive and active-passive tip displacement responses are depicted in Figures 5.47 and 5.48 with $E_{\text {avg }}$, respectively. Each response within these figures, (a), (b), and (c) has $E_{\text {avg }}$ of $200 \mathrm{GPa}$, but with different $E_{M}$ and $E_{C}$ values. Figure 5.49 is the actuation voltage response of the active-passive damping, which is proportional to the displacement response. For part (a) of all the responses, the Q2DT E-FGM and homogeneous response almost match exactly, while the Q2DT P-FGM response has a slight phase shift from the two. As the $\Delta E$ increases in part (b), the responses are out of phase from each other. Also, the Q2DT E-FGM response decreased in transverse displacement, while the Q2DT P-FGM response increased in transverse displacement compared to part (a). Furthermore, in part (c), the same observation from part (b) is seen, but with more decrease and increase in transverse displacement, for the E-FGM and P-FGM responses, respectively. From Figure 5.48, the response that has the best damping capabilities is the Q2DT E-FGM in part (c), this has the biggest $\Delta E$. 


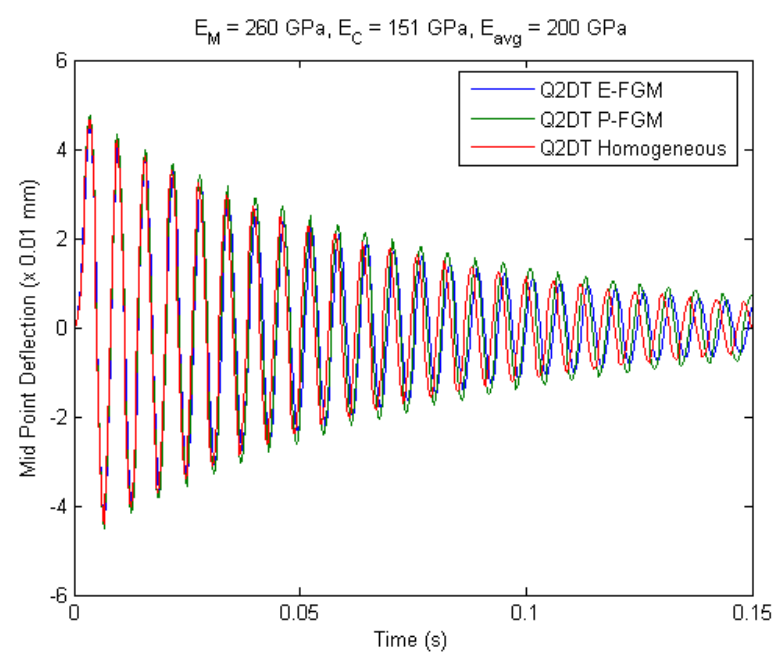

(a)

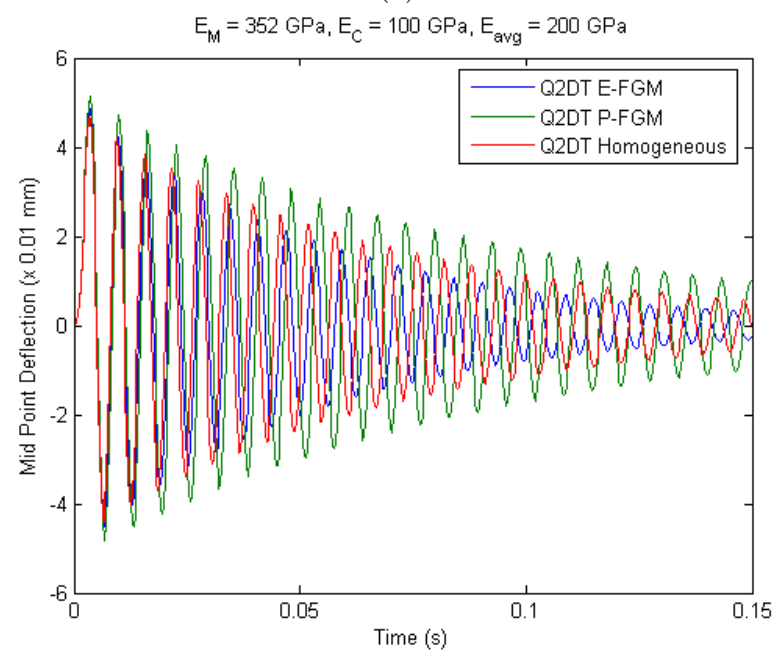

(b)

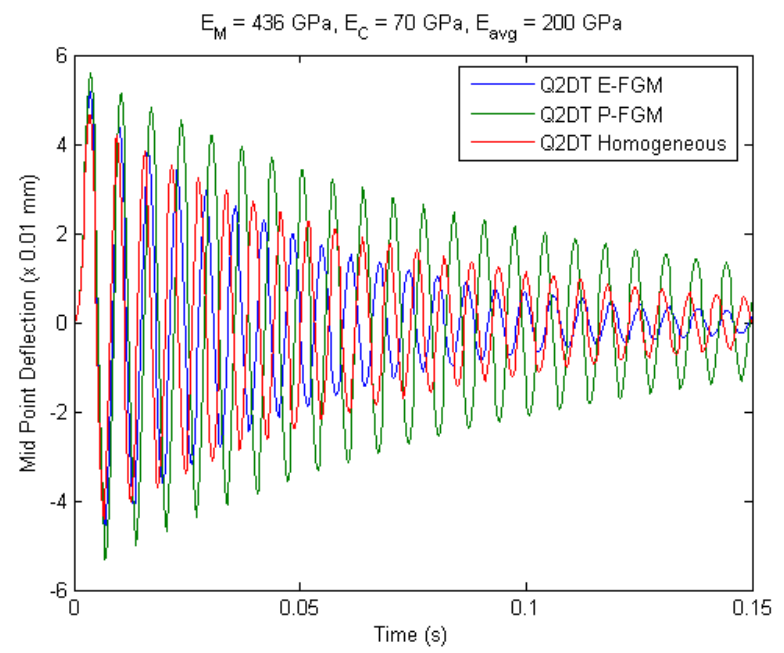

$(c)$

Figure 5.47: Effect of varying constituents moduli for fixed average elastic modulus on the mid-point transverse displacement for uncontrolled-passive damping of partially clampedclamped beam. (a),(b),(c) $E_{\text {avg }}=200 \mathrm{GPa}$ for all. 


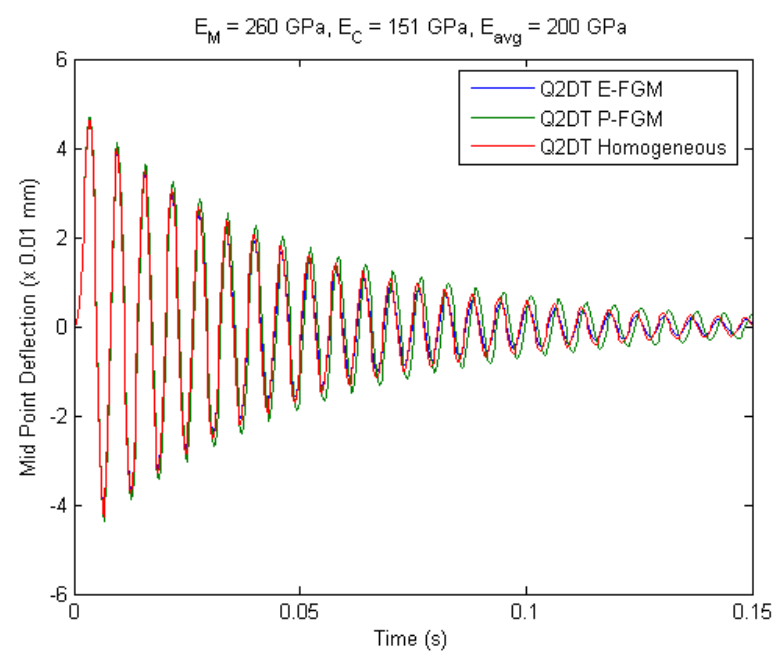

$(a)$

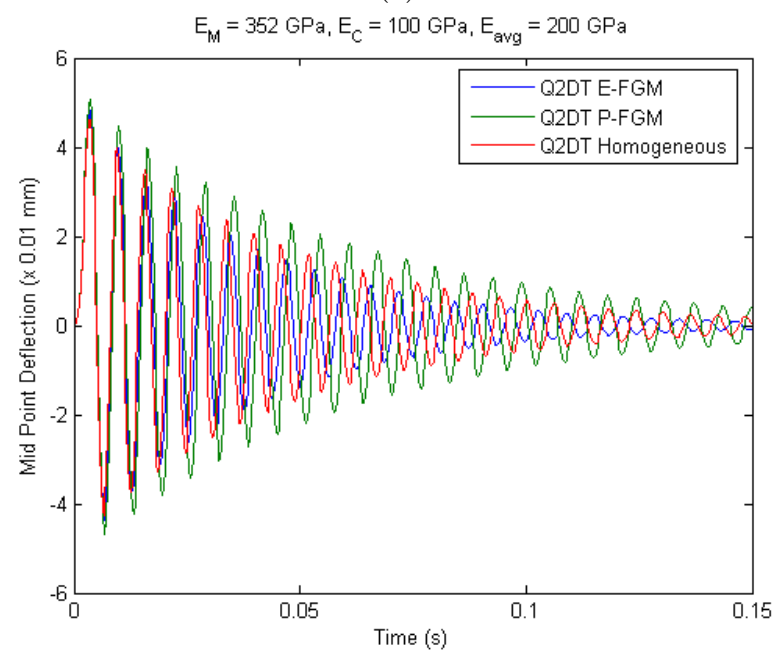

$(b)$

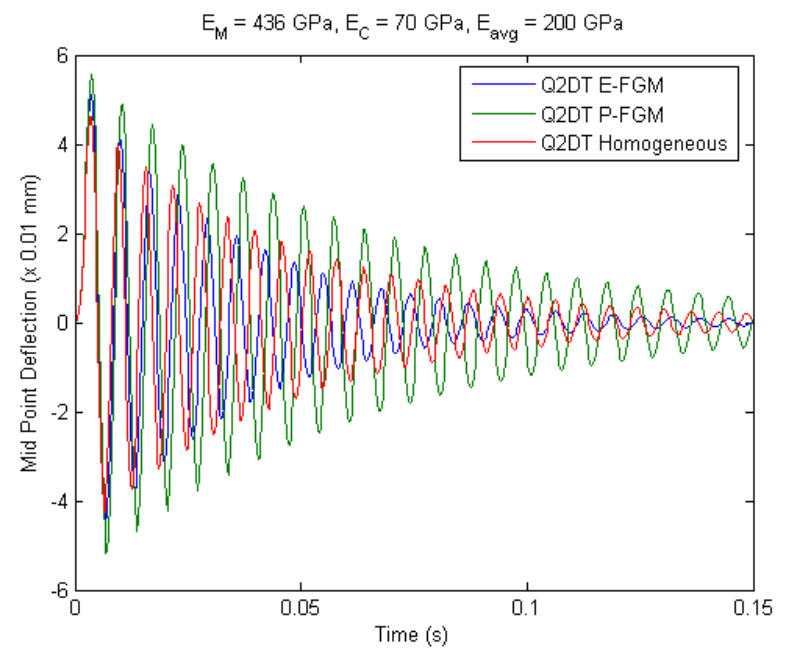

(c)

Figure 5.48: Effect of varying constituents moduli for fixed average elastic modulus on the mid-point transverse displacement for controlled-active-passive damping of partially clampedclamped beam. (a),(b),(c) $E_{\text {avg }}=200 \mathrm{GPa}$ for all. 


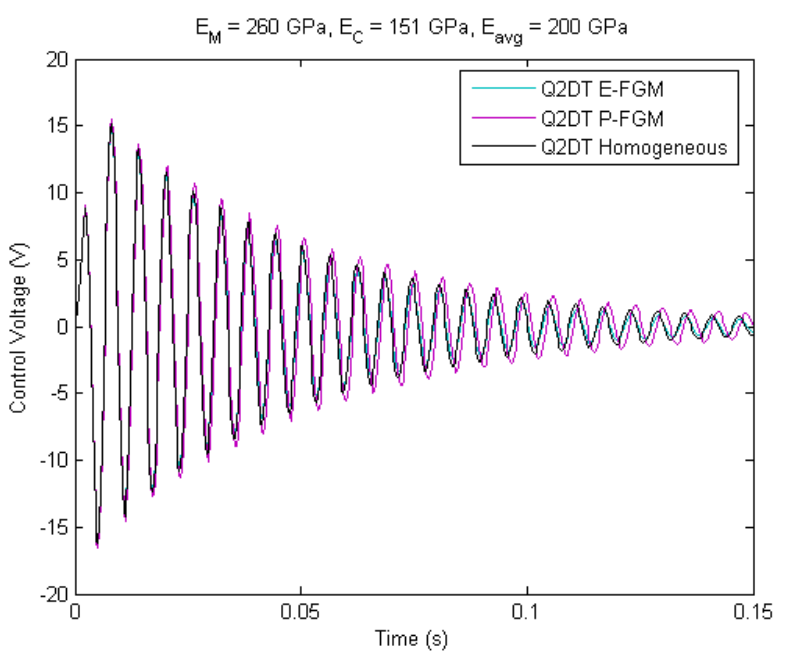

(a)

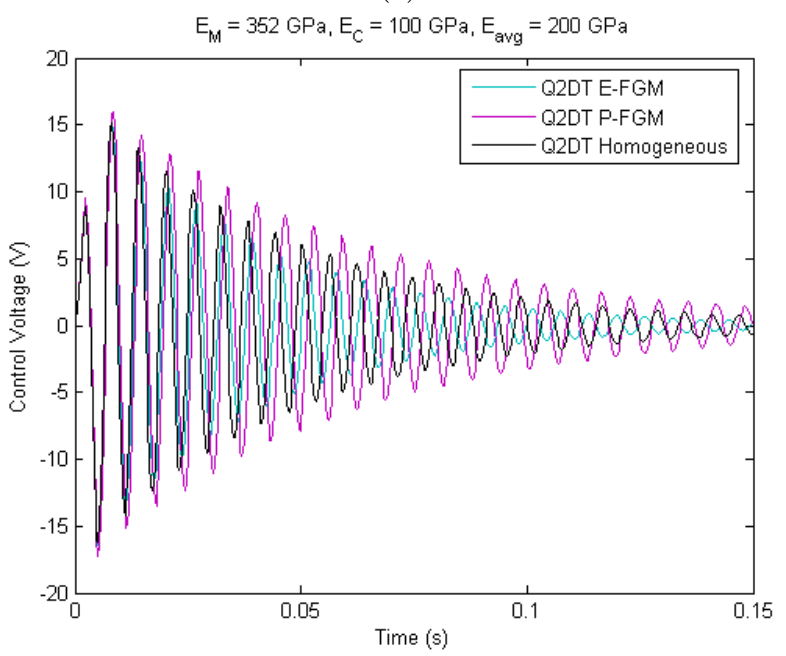

(b)

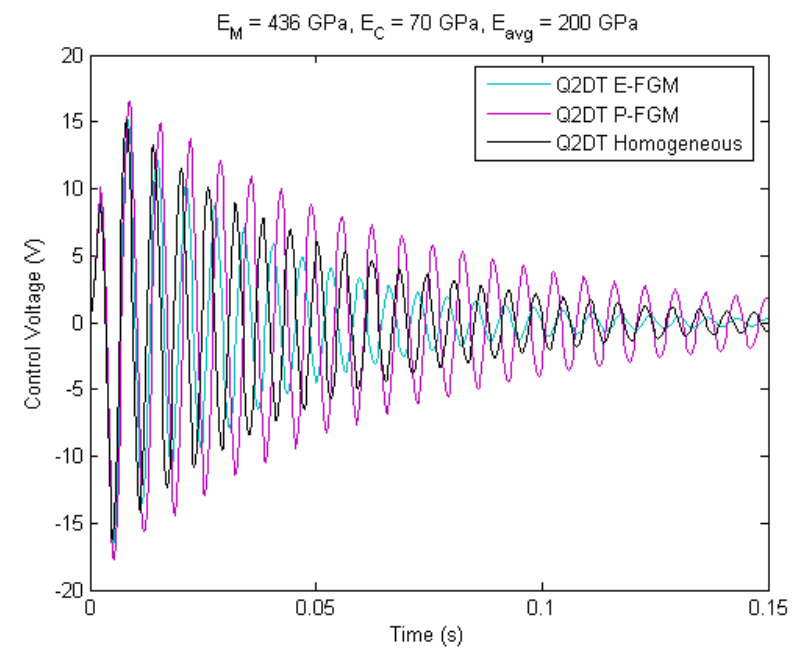

$(c)$

Figure 5.49: Effect of varying constituents moduli for fixed average elastic modulus on the actuation voltage for controlled-active-passive damping of partially clamped-clamped beam. (a),(b),(c) $E_{a v g}=200 \mathrm{GPa}$ for all. 
(iii) Effect of exchange in the metal to ceramic and ceramic to metal elastic moduli:

The uncontrolled-passive damping responses for the exchange of metal to ceramic and ceramic to metal elastic moduli are depicted in Figure 5.50. The same observations are made from the cantilevered problems. The first plot, with $\Delta E=109 \mathrm{GPa}$, part (a), has responses that almost corroborate perfectly for both the Q2DT E-FGM and P-FGM responses. With respect to part (b), two responses with the smallest transverse displacement (Q2DT E-FGM $\left(E_{M}>E_{C}\right)$ and Q2DT P-FGM $\left.\left(E_{M}<E_{C}\right)\right)$ match each other. The two responses with the largest transverse displacement (Q2DT P-FGM $\left(E_{M}>E_{C}\right)$ and Q2DT E-FGM $\left(E_{M}<E_{C}\right)$ ) result in the same peak-to-peak amplitude, but have different phase shift. A larger phase shift for both the smaller and larger displacement responses are seen from part (b) to part (c). As $\Delta E$ increases, the Q2DT P-FGM $\left(E_{M}>E_{C}\right)$ and Q2DT E-FGM $\left(E_{M}<E_{C}\right)$ responses continue to increase in amplitude, while the Q2DT E-FGM $\left(E_{M}>E_{C}\right)$ and Q2DT P-FGM $\left(E_{M}<E_{C}\right)$ responses decrease in amplitude in comparison to the homogeneous response. The responses that have the best damping capabilities are the Q2DT E-FGM $\left(E_{M}>E_{C}\right)$ and Q2DT P-FGM $\left(E_{M}<E_{C}\right)$ responses. 


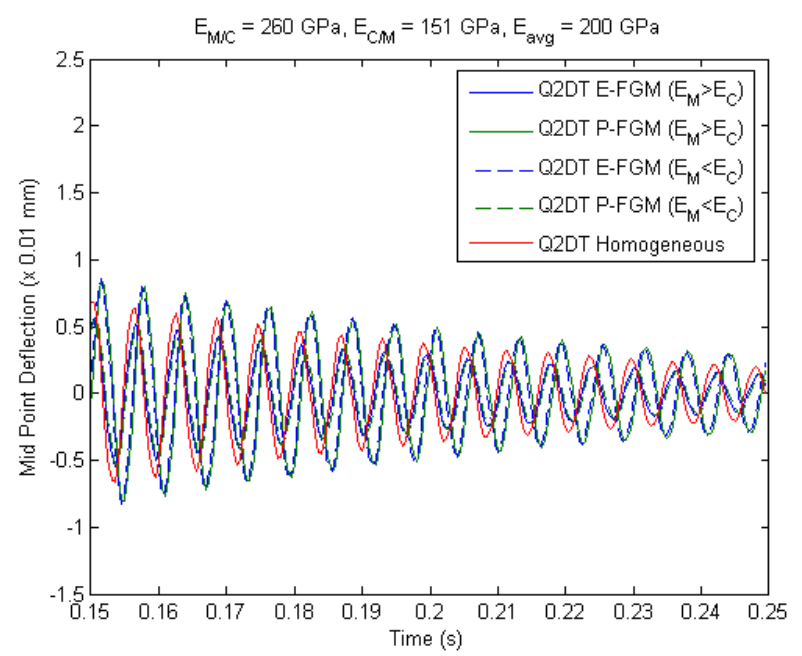

(a)

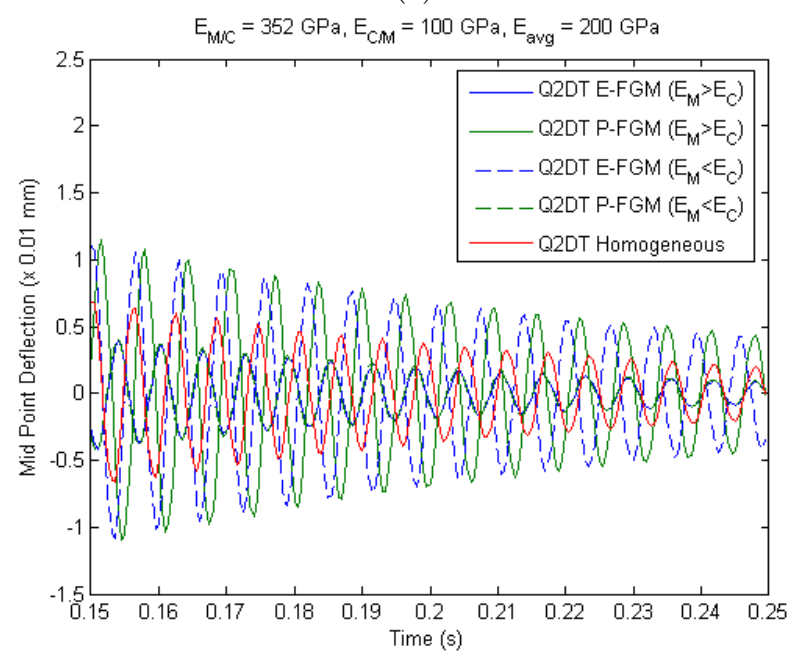

$(b)$

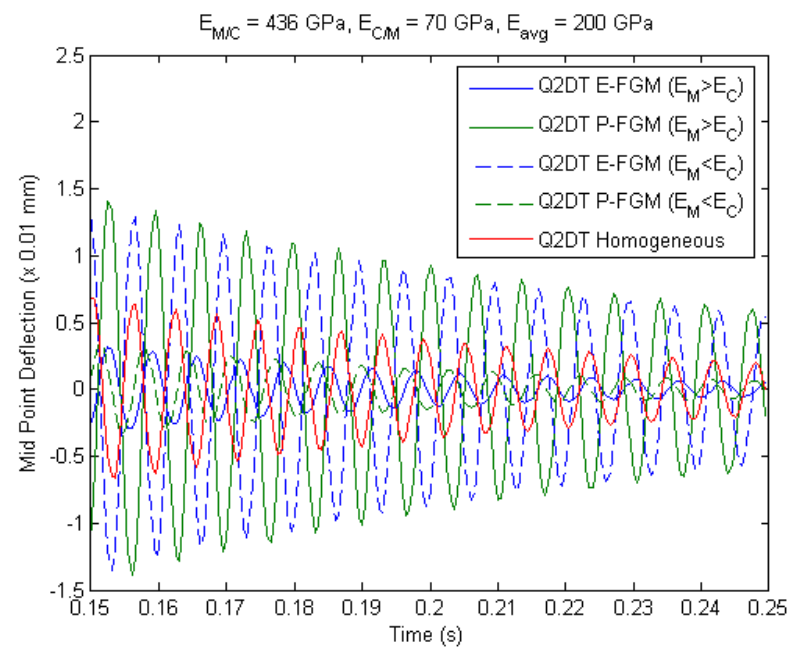

(c)

Figure 5.50: Effect of exchange in metal and ceramic elastic moduli on the mid-point transverse displacement for uncontrolled-passive damping of partially clamped-clamped beam. (a),(b),(c) $E_{a v g}=200 \mathrm{GPa}$ for all. 


\subsubsection{Simply-supported Beam Case}

The same simply-supported beam depicted in Figure 5.30 and its parameters from Section 5.2.1 are utilized in this section.

(i) Effect of change in the average elastic modulus:

Figures 5.51 and 5.52 depict the responses of the beam when it is passively and actively damped, respectively. Figure 5.53 shows the actuator voltage response when the system gain is $K_{v}=350 \mathrm{~V} /(\mathrm{m} / \mathrm{s})$ as indicated in the previous sections. The plot for part (a) has responses that are almost exact with extremely small phase shifts. Just like the other beam cases, as $E_{\text {avg }}$ increases, the Q2DT E-FGM response becomes more damped, decreasing in transverse displacement, whereas, the Q2DT P-FGM response increases in transverse displacement. However, the progression in transverse displacement is not as prominent as in the other beam cases. Also, as $E_{\text {avg }}$ increases, the responses slowly become more out of phase with each other. An observation between the $E_{\text {avg }}$ 's from parts (a) to (c) shows that the final amplitudes are generally the same. The responses with a higher $E_{\text {avg }}$ start with a smaller amplitude than that of a lower $E_{\text {avg }}$, but both dampen to the same amplitude. The actuation voltage is proportional to the active-passive deflection. Also, as observed for previous cases, the frequency of vibration increases as $E_{\text {avg }}$ increases. In comparison to the partially clamped-clamped beam case, the simply-supported beam experiences higher amplitudes of vibration, but reduced frequency. 


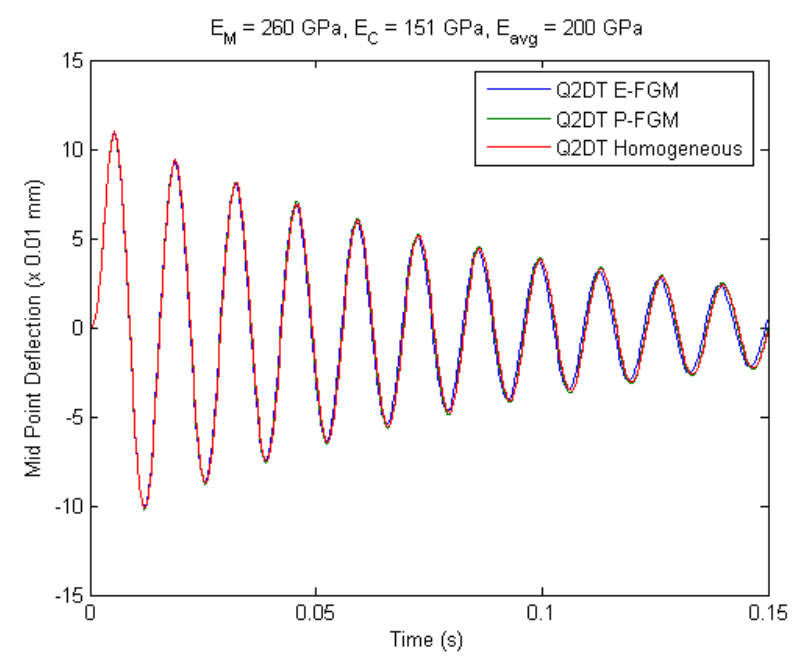

(a)

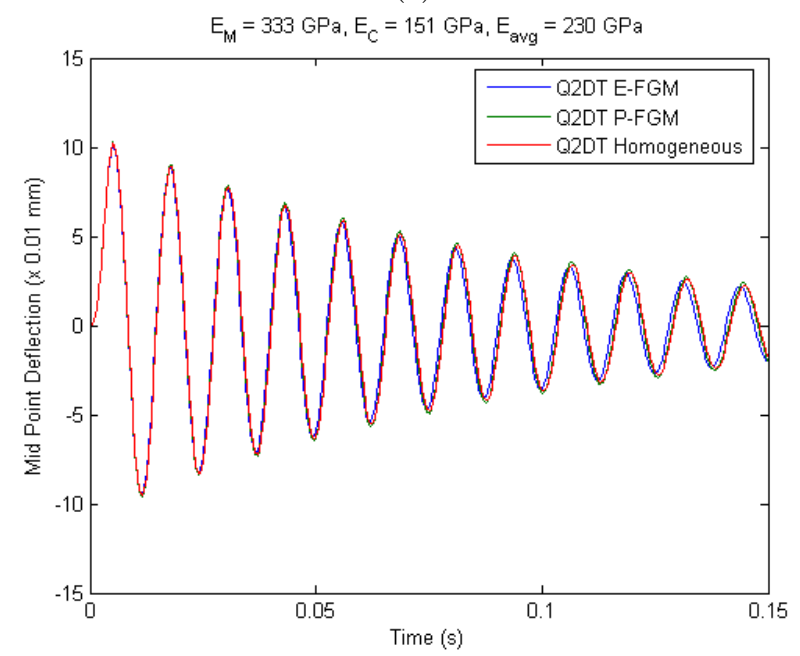

$(b)$

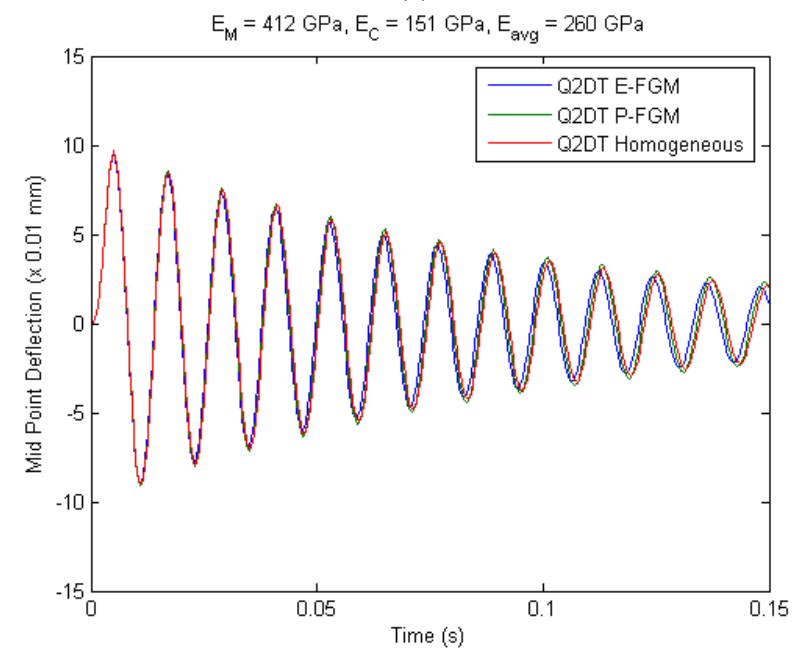

(c)

Figure 5.51: Effect of change in average elastic modulus on the mid-point transverse displacement for uncontrolled-passive damping of simply-supported beam. (a),(b),(c) $E_{a v g}=200$ GPa, $230 \mathrm{GPa}$, and $260 \mathrm{GPa}$. 


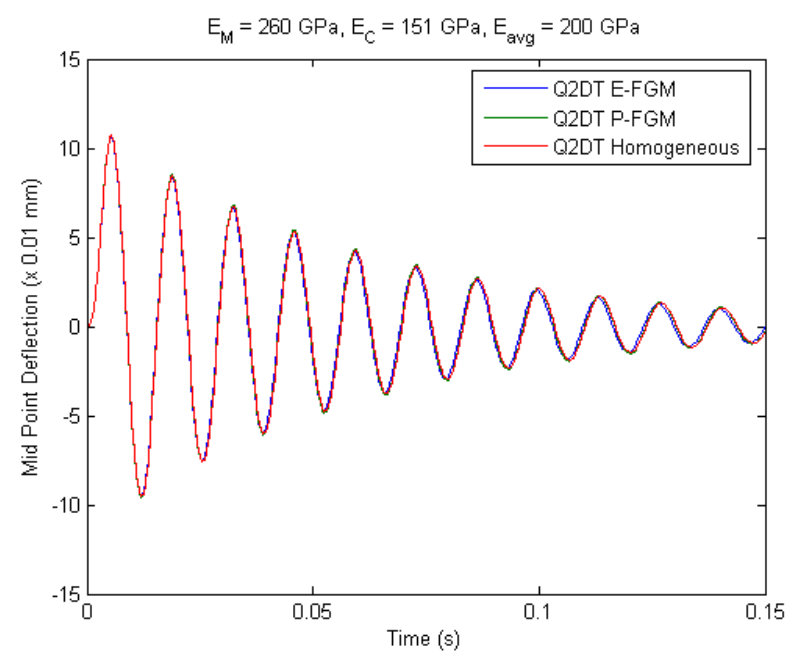

(a)

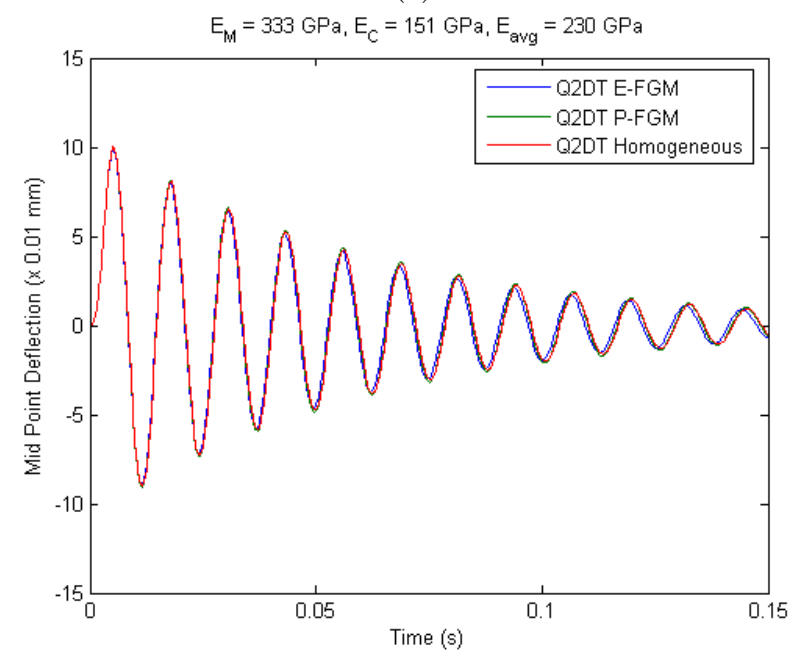

$(b)$

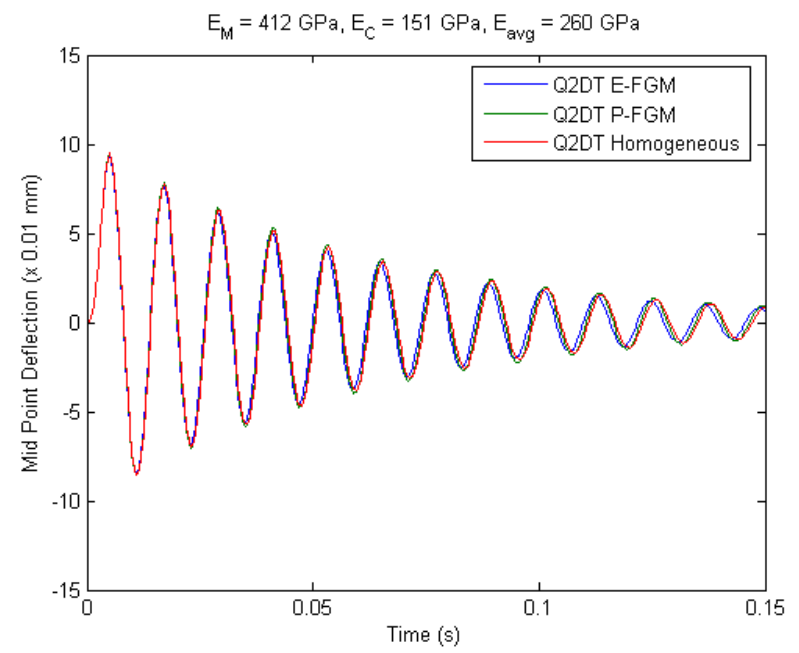

(c)

Figure 5.52: Effect of change in average elastic modulus on the mid-point transverse displacement for controlled-active-passive damping of simply-supported beam. (a),(b),(c) $E_{\text {avg }}=$ $200 \mathrm{GPa}, 230 \mathrm{GPa}$, and $260 \mathrm{GPa}$. 


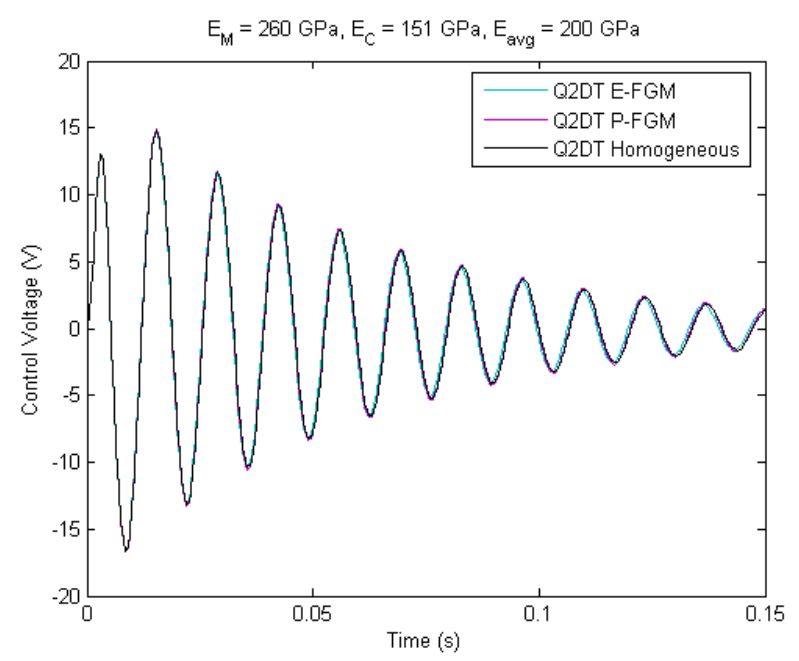

(a)

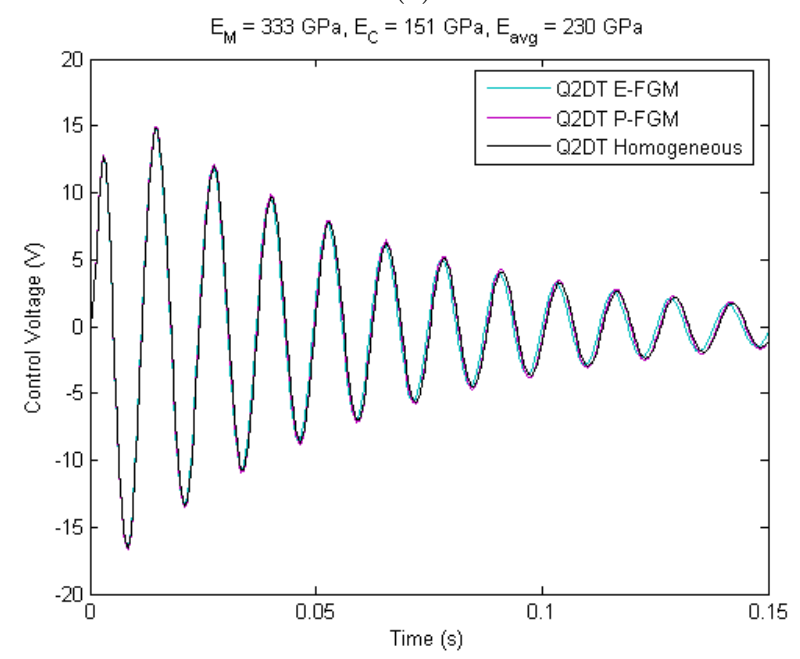

$(b)$

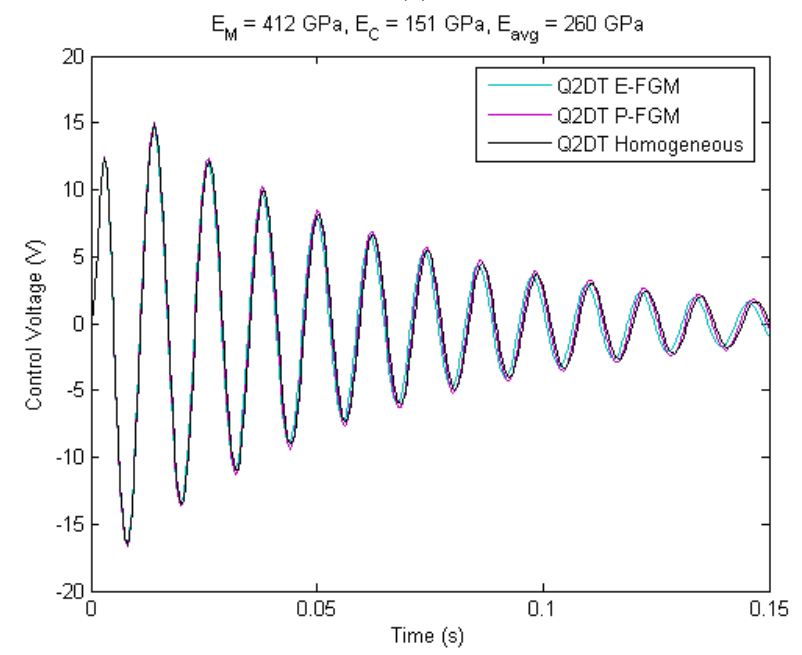

(c)

Figure 5.53: Effect of change in average elastic modulus on the actuation voltage for controlled-active-passive damping of simply-supported beam. (a),(b),(c) $E_{\text {avg }}=200 \mathrm{GPa}$, $230 \mathrm{GPa}$, and $260 \mathrm{GPa}$. 
(ii) Effect of varying constituents moduli for fixed average elastic modulus:

Figures 5.54 and 5.55 depict the passive and active-passive tip displacement responses when $E_{a v g}=200 \mathrm{GPa}$. The active-passive actuation voltage response is shown in Figure 5.56. As $\Delta E$ increases, the Q2DT E-FGM response progressively dampens, where the best damping is observed at part (c) Figure 5.55, $\Delta E=366 \mathrm{GPa}$. Due to the nature of the power-law function, as $\Delta E$ increases, the Q2DT P-FGM response gradually increases in amplitude. Unlike the other beam cases, the simply-supported case has passive and activepassive tip displacement responses that do not follow the same pattern. For parts (b) and (c), the passive-damped beams generally have responses that are in phase to one another, but for the active-passive damped scenarios, the Q2DT P-FGM responses do not damp as well as the Q2DT E-FGM responses. Also, the active-passive damping responses become out-of-phase from one another. The actuation voltage produces the same pattern as the active-passive response because they are directly proportional to each other. 


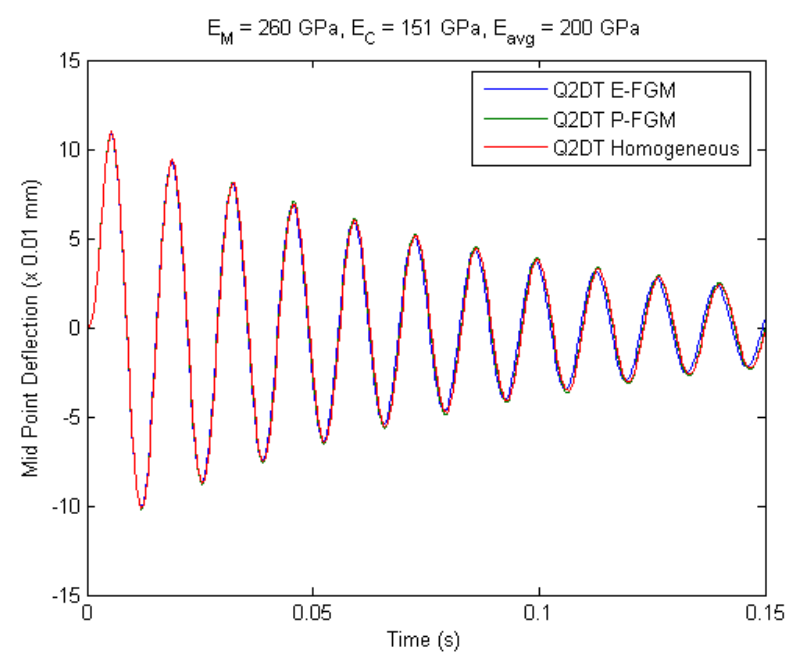

(a)

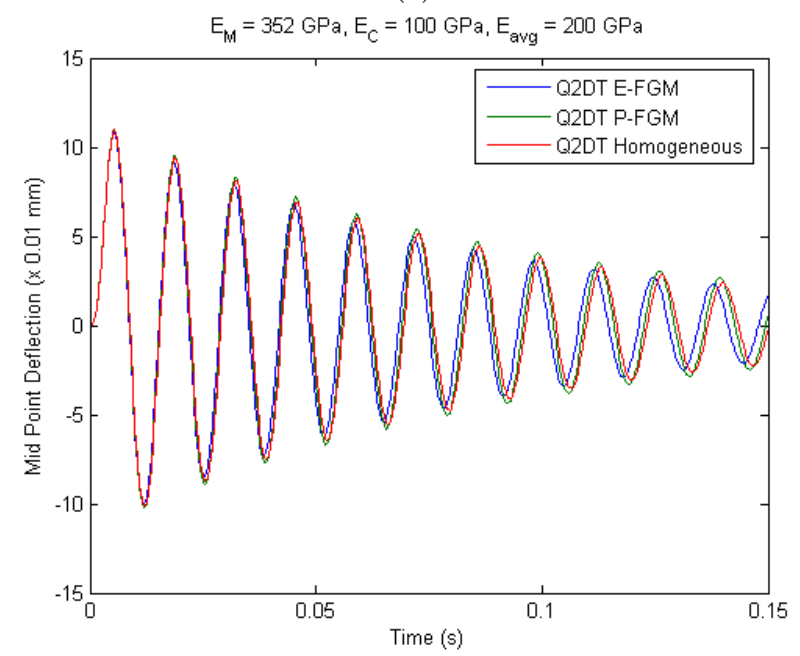

$(b)$

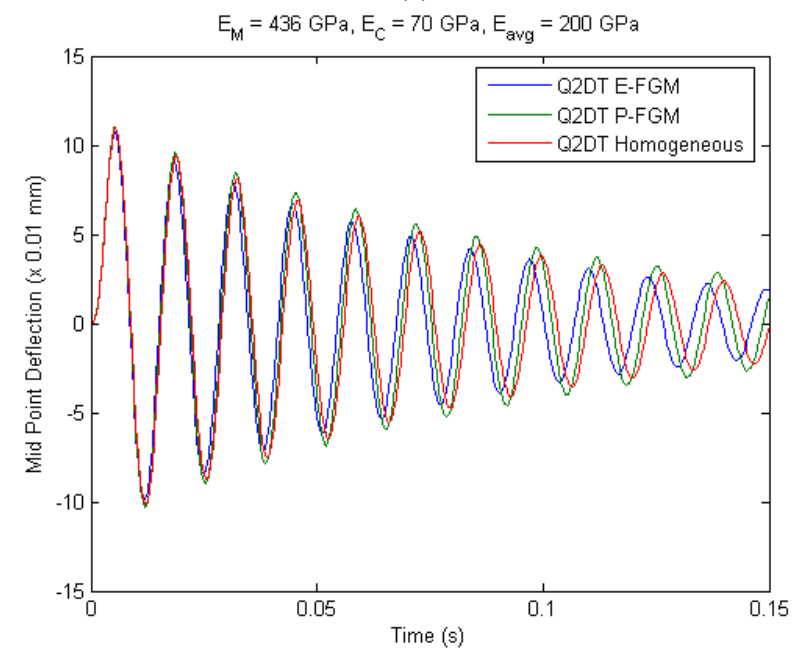

(c)

Figure 5.54: Effect of varying constituents moduli for fixed average elastic modulus on the mid-point transverse displacement for uncontrolled-passive damping of simply-supported beam. (a),(b),(c) $E_{a v g}=200 \mathrm{GPa}$ for all. 


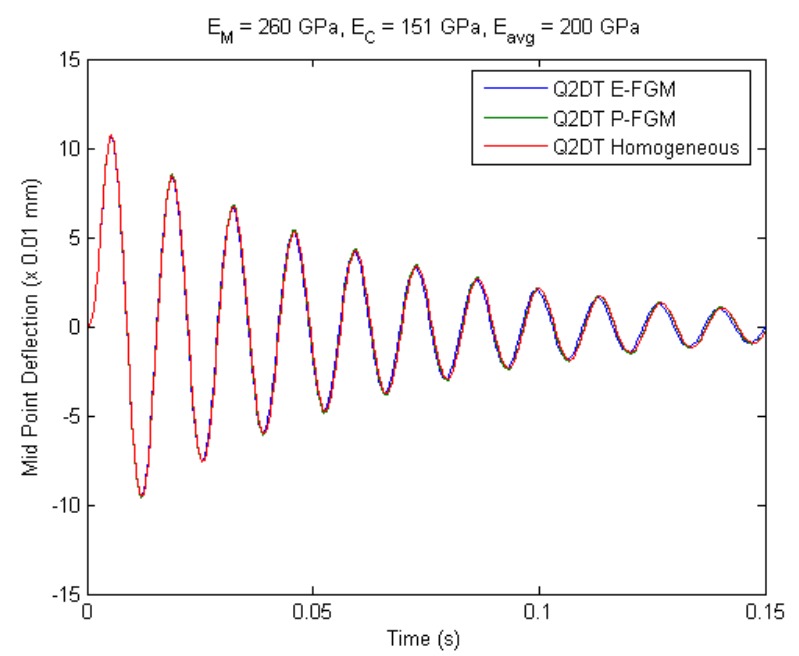

(a)

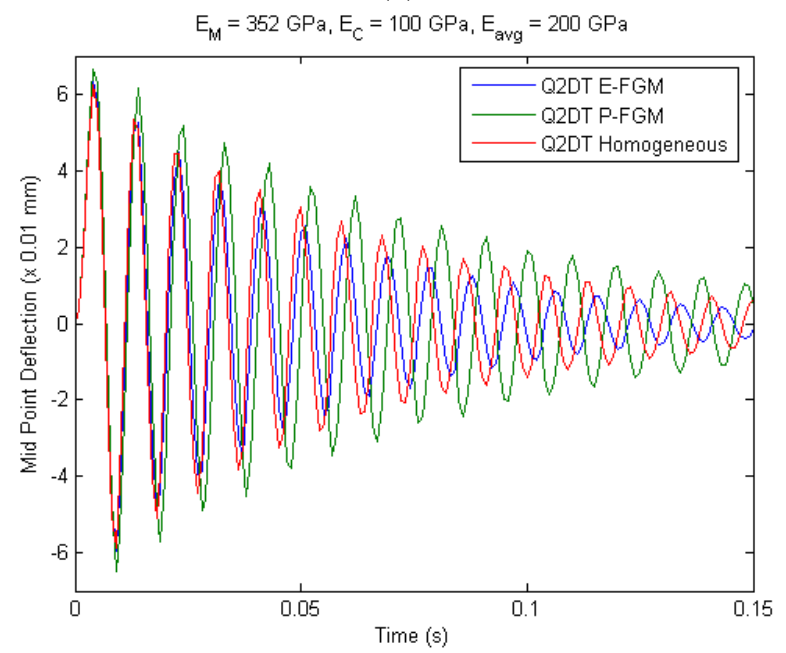

$(b)$

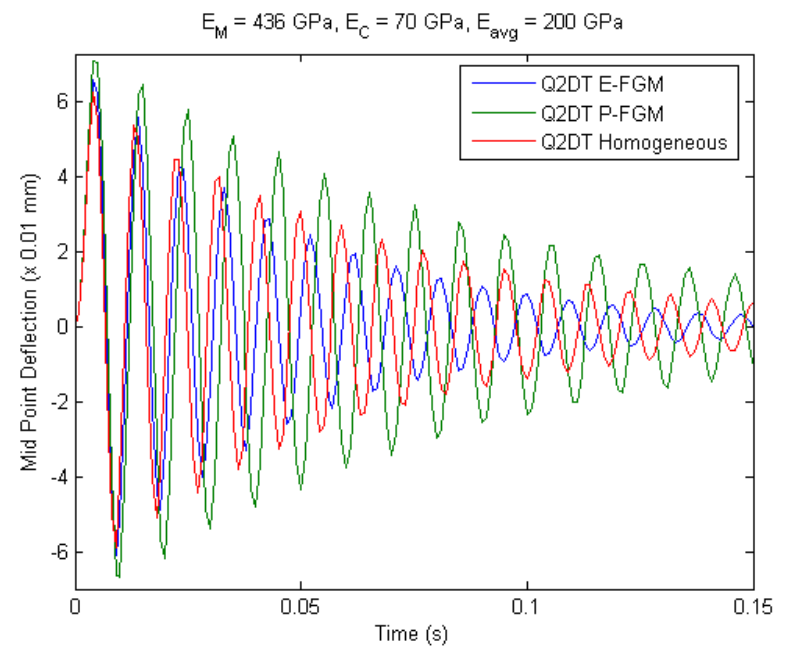

(c)

Figure 5.55: Effect of varying constituents moduli for fixed average elastic modulus on the mid-point transverse displacement for controlled-active-passive damping of simply-supported beam. (a),(b),(c) $E_{\text {avg }}=200 \mathrm{GPa}$ for all. 


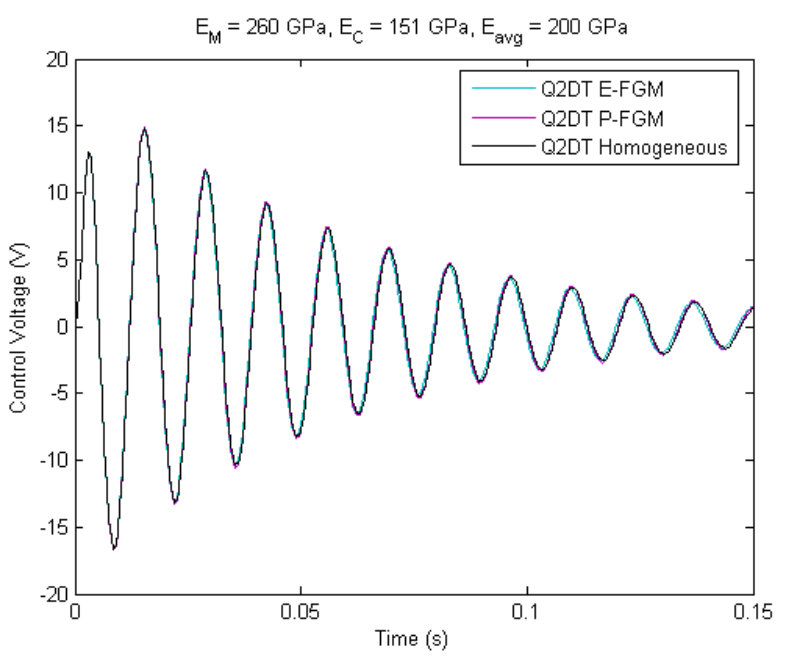

$(a)$

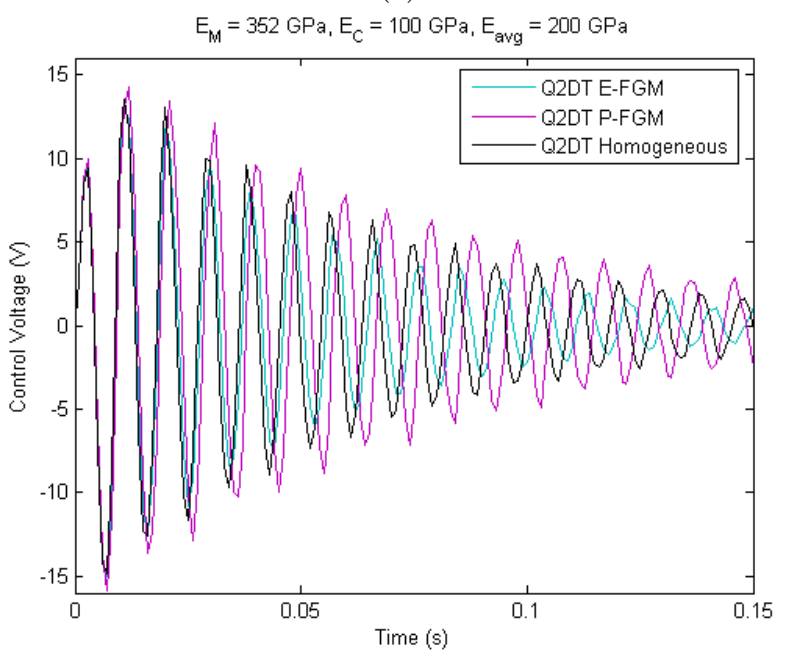

$(b)$

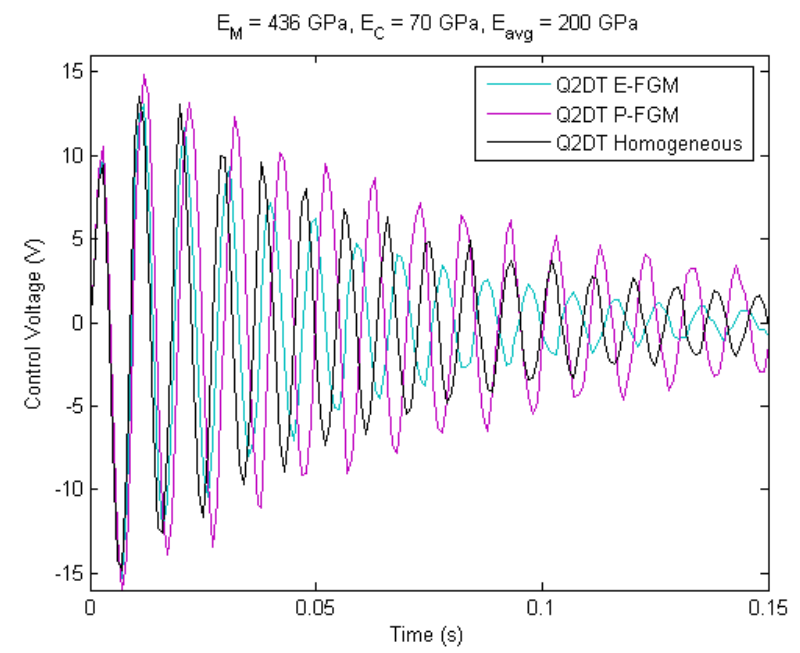

(c)

Figure 5.56: Effect of varying constituents moduli for fixed average elastic modulus on the actuation voltage for controlled-active-passive damping of simply-supported beam. (a),(b),(c) $E_{\text {avg }}=200 \mathrm{GPa}$ for all. 
(iii) Effect of exchange in the metal to ceramic and ceramic to metal elastic moduli:

Figure 5.57 depicts the response for the passive damping of the exchange of metal to ceramic and ceramic to metal elastic moduli. Similar observations are made from the partially clamped-clamped problem. A comparison between the two (partially clamped-clamped and simply-supported) shows that the simply-supported response has lower frequency and larger peak-to-peak amplitude of vibration than the partially clamped-clamped case. In part (a) of Figure 5.57, the Q2DT E-FGM $\left(E_{M}>E_{C}\right)$ and Q2DT P-FGM $\left(E_{M}<E_{C}\right)$ responses as well as the Q2DT P-FGM $\left(E_{M}>E_{C}\right)$ and Q2DT E-FGM $\left(E_{M}<E_{C}\right)$ responses match perfectly. The Q2DT E-FGM $\left(E_{M}>E_{C}\right)$ and Q2DT P-FGM $\left(E_{M}<E_{C}\right)$ responses are exactly in phase with the homogeneous response, but have slightly smaller transverse displacement. With increasing $\Delta E$, the responses that matched in part (a) no longer match in parts (b) and (c); they experience larger phase shifts. Also, for parts (b) and (c), there are no responses that are in phase with the homogeneous response. The Q2DT E-FGM $\left(E_{M}>E_{C}\right)$ and Q2DT P-FGM $\left(E_{M}<E_{C}\right)$ responses at the highest $\Delta E$ in part (c) result in the best damping capabilities. 


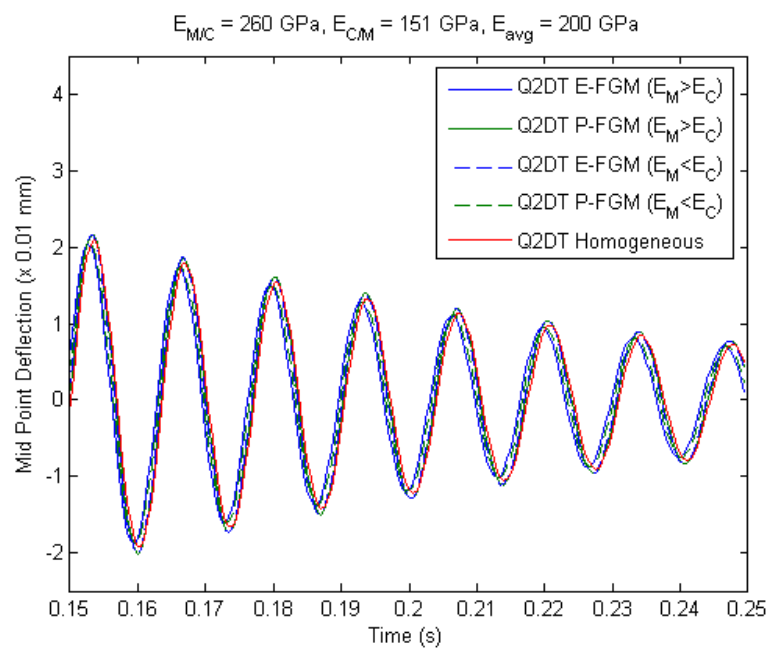

(a)

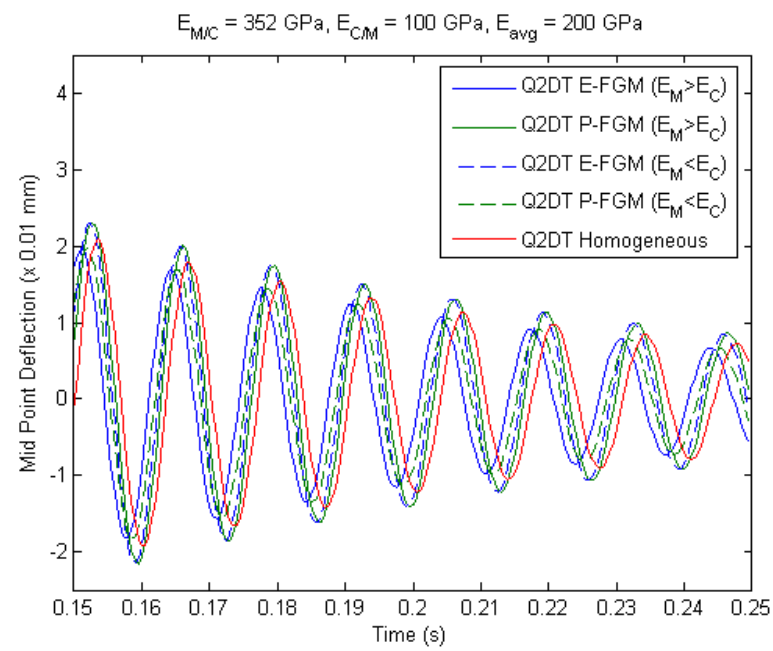

$(b)$

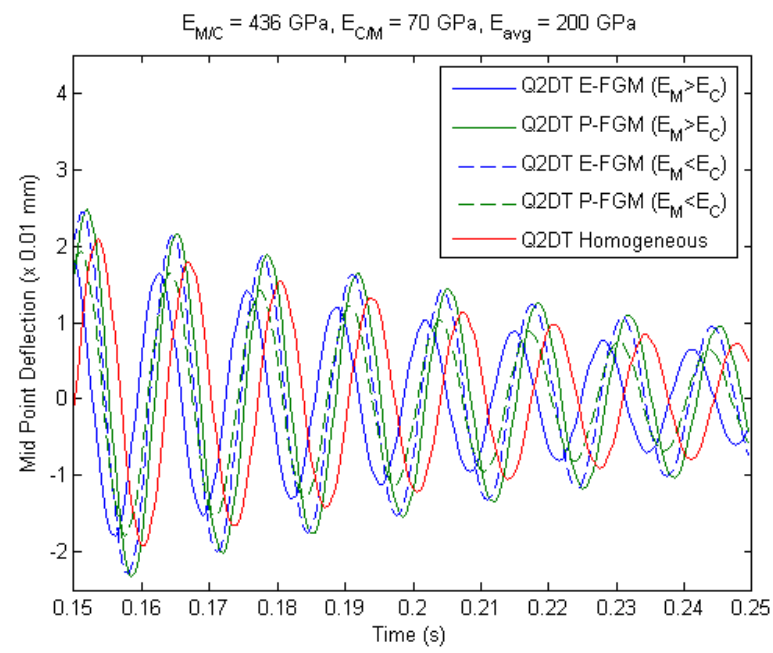

(c)

Figure 5.57: Effect of exchange in metal and ceramic elastic moduli on the mid-point transverse displacement for uncontrolled-passive damping of simply-supported beam. (a),(b),(c) $E_{a v g}=200 \mathrm{GPa}$ for all. 


\section{Chapter 6}

\section{Summary, Conclusions and Recommendations}

A quasi-2D finite element formulation is presented in the previous chapters for the vibration analysis of a functionally graded (FG) host beam with an active-passive constraining layer damping (ACLD) treatment. The ACLD treatment is composed of a viscoelastic layer that passively damps the structure and a piezoelectric fiber-reinforced composite (PFRC) laminate that actively constrains the layer.

The PFRC laminate acts as the actuator of the system through a velocity feedback control system. The PFRC laminate consists of four plies that are oriented at an angle $\theta$ in the reference plane of the system. The ABD constitutive matrix, which relates force and moment resultants with strains and curvatures, is determined through the use of the classical lamination theory (CLT). The host beam is taken to have a Young's modulus that is varying along the thickness of the beam, while the Poisson's ratio and density are constant throughout the beam. The viscoelastic material time-dependent behavior is described by utilizing a four-parameter fractional derivative model.

The formulation of the three-layer beam system is developed as a quasi-2D Euler Bernoulli (Q2DE) and quasi-2D Timoshenko (Q2DT). The Q2DE is a model whereby the top PFRC laminate and bottom host beam follow the Euler-Bernoulli beam hypotheses and the viscoelastic core follows a higher-order beam theory. Similarly, the Q2DT is a model where the top PFRC laminate and bottom host beam are formulated as Timoshenko beams and the viscoelastic core remains based on a higher-order beam theory. The deformation kinematics of the top constraining layer and bottom host beam are such that the axial displacements are linear in the thickness coordinate and transverse displacements are constant. In the viscoelastic core, the axial displacement varies cubically, while the transverse displacement varies quadratically. Although this model has a smaller amount of degrees of freedom (DOF) 
than the previous work done by Ref. [3], it is highly accurate. The present quasi-2D formulation is significantly simplified while maintaining the same level of accuracy.

Three examples from the literature are utilized to determine the efficiency of this current formulation: a sandwich beam with functionally graded (FG) core, a visco-aluminum sandwich beam and a cantilevered beam with viscoelastic core and piezoelectric constraining layer. Throughout the parametric study, the active-passive constraining layer continuously showed superior damping capabilities over the passive damping. Four boundary conditions were analyzed: fully-clamped cantilevered, partially-clamped cantilevered, partially clamped-clamped and simply-supported beams. Two studies were performed to examine the effect of ply orientation and the effect of FGM properties.

During the effect of ply orientation analysis, it was observed that the passively damped fully-clamped cantilevered beam with a stacking sequence of $0_{4}^{\circ}$ resulted in the best damping rate because its stiffness causes the greatest shear deformation of the viscoelastic core. In the active-passive scenario, however, this stiffness works against the active control effort of the actuator; hence, the $\left[0^{\circ} / 90^{\circ}\right]_{S}$ is better complemented and provides the best attenuation rate. For the partially-clamped cantilevered beam case, active-passive damping has a very slow attenuation rate compared to the fully-clamped cantilevered beam. In the partially clampedclamped case, the vibration pattern was similar to the clamped cantilevered cases, but with smaller amplitude and higher frequency. The simply-supported beam responses have larger amplitude and lower frequency compared to the partially clamped-clamped responses.

In the study of the effect of FGM properties on the host beam, three scenarios were examined: (i) the effect of change in average elastic moduli, (ii) the effect of varying constituents moduli for fixed average elastic moduli, and (iii) the effect of exchange in the metal to ceramic and ceramic to metal elastic moduli. It was observed that all four boundary conditions yielded the same response patterns, but with different ranges in actuation.

In the first scenario, with increasing $E_{\text {avg }}$, the Q2DT E-FGM response slightly decreased in transverse displacement and the Q2DT P-FGM displacement scarcely changed. Also, as $E_{\text {avg }}$ increased, the frequency of each response increased. Out of the four beam cases, the simply-supported beam yielded the most uniform displacement for all formulation responses (Q2DT E-FGM, P-FGM, and homogeneous).

With respect to part (ii) of the FGM analysis such that the average Young's moduli $E_{\text {avg }}$ were fixed to $200 \mathrm{GPa}$, as $\Delta E$ increased, the transverse displacement of the Q2DT EFGM response gradually decreased while the Q2DT P-FGM response increased. The Q2DT E-FGM response with the largest $\Delta E$ yielded the best damping rate.

For part (iii), as $\Delta E$ increased, the Q2DT E-FGM $\left(E_{M}>E_{C}\right)$ and P-FGM $\left(E_{M}<E_{C}\right)$ responses gradually decreased as the Q2DT P-FGM $\left(E_{M}>E_{C}\right)$ and E-FGM $\left(E_{M}<E_{C}\right)$ 
responses increased. At the largest $\Delta E$, the response that yielded the smallest peak-to-peak amplitude of vibration was the Q2DT E-FGM $\left(E_{M}>E_{C}\right)$ response followed very closely by the P-FGM $\left(E_{M}<E_{C}\right)$ response. These observations suggest that, for a host beam with $100 \%$ metal composition at the top and $100 \%$ ceramic composition at the bottom of the beam, using the E-FGM distribution demands that the material with the highest elastic modulus $\left(E_{M}\right.$ when $E_{M}>E_{C}$ ) be placed at the top of the host beam to obtain the least amount of vibration. Also, the use of the P-FGM distribution in a FG host beam requires that the material with the lowest elastic modulus $\left(E_{M}\right.$ when $\left.E_{M}<E_{C}\right)$ should be situated at the top of the beam in order to obtain the best damping.

Even though the current study is quite thorough, there is always room for improvement, such as the utilization of other materials to enhance the structure. Further extension of this work could possibly aid in the solution to noise and vibration problems. Future investigations may include:

1. The present formulation can progress to include the concept of plates and/or shells. A quasi-3D finite element model such as that seen in Ref. [9] can be utilized.

2. The use of different viscoelastic models can be examined. This thesis uses the fourparameter fractional derivative model which could not be directly replicated using commercial finite element software such as ANSYS which employs the Prony series method to represent viscoelastic material.

3. The viscoelastic constraining layer could incorporate an FG property where its elastic modulus varies throughout the thickness. The effectiveness of the active constraining layer might be improved since it would be bonded to the surface of the passive viscoelastic layer where there will be less stiffness and, thus, increase the actuation of the beam.

4. The presence of imperfect bonding can be explored.

5. The current formulation can be verified experimentally by performing vibration simulation tests. 


\section{Appendix A}

\section{Appendix}

\section{A.1 MATLAB Codes}

The following MATLAB code was used to find response of an active constrained-layer damped (ACLD) functionally-graded (FG) beam under a triangular impulse load.

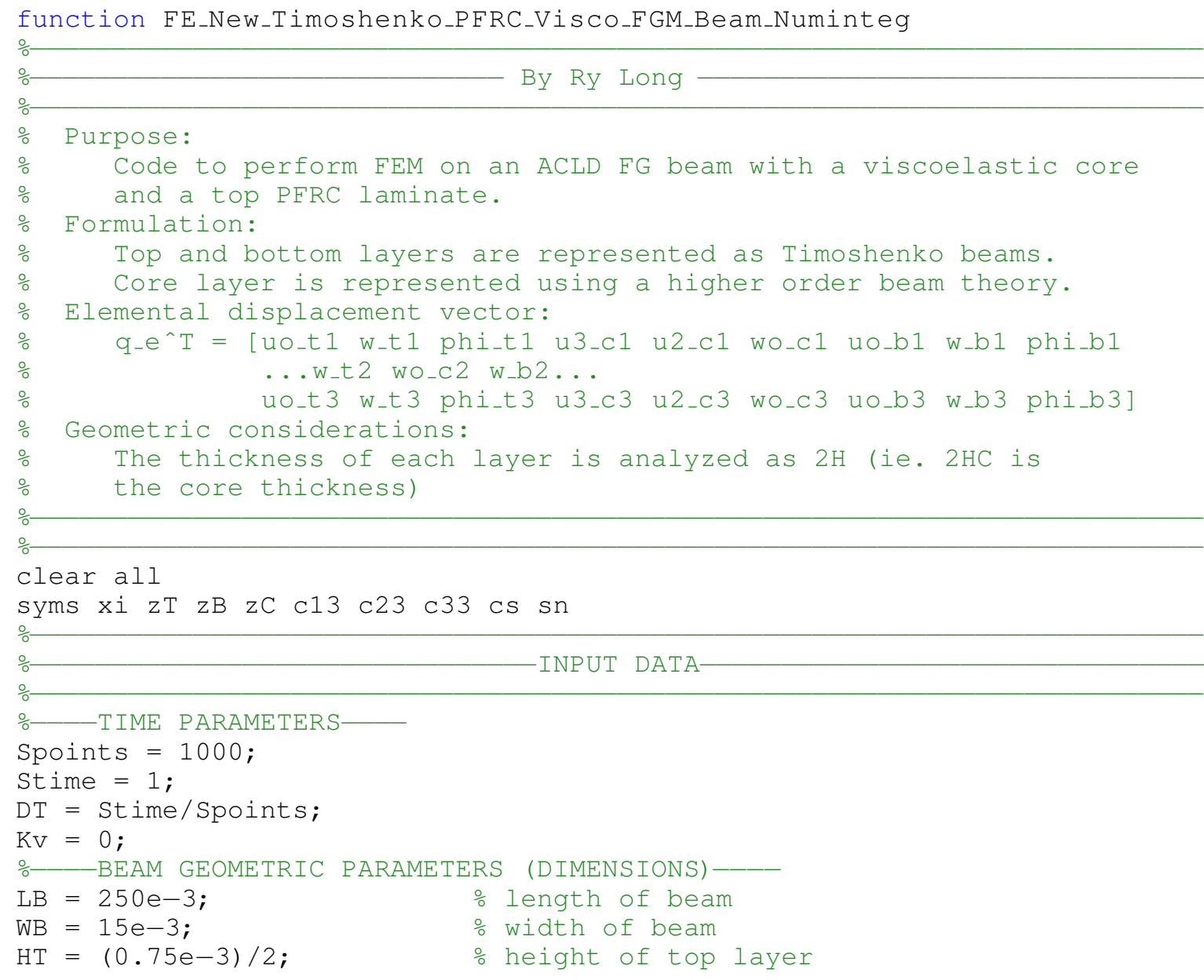




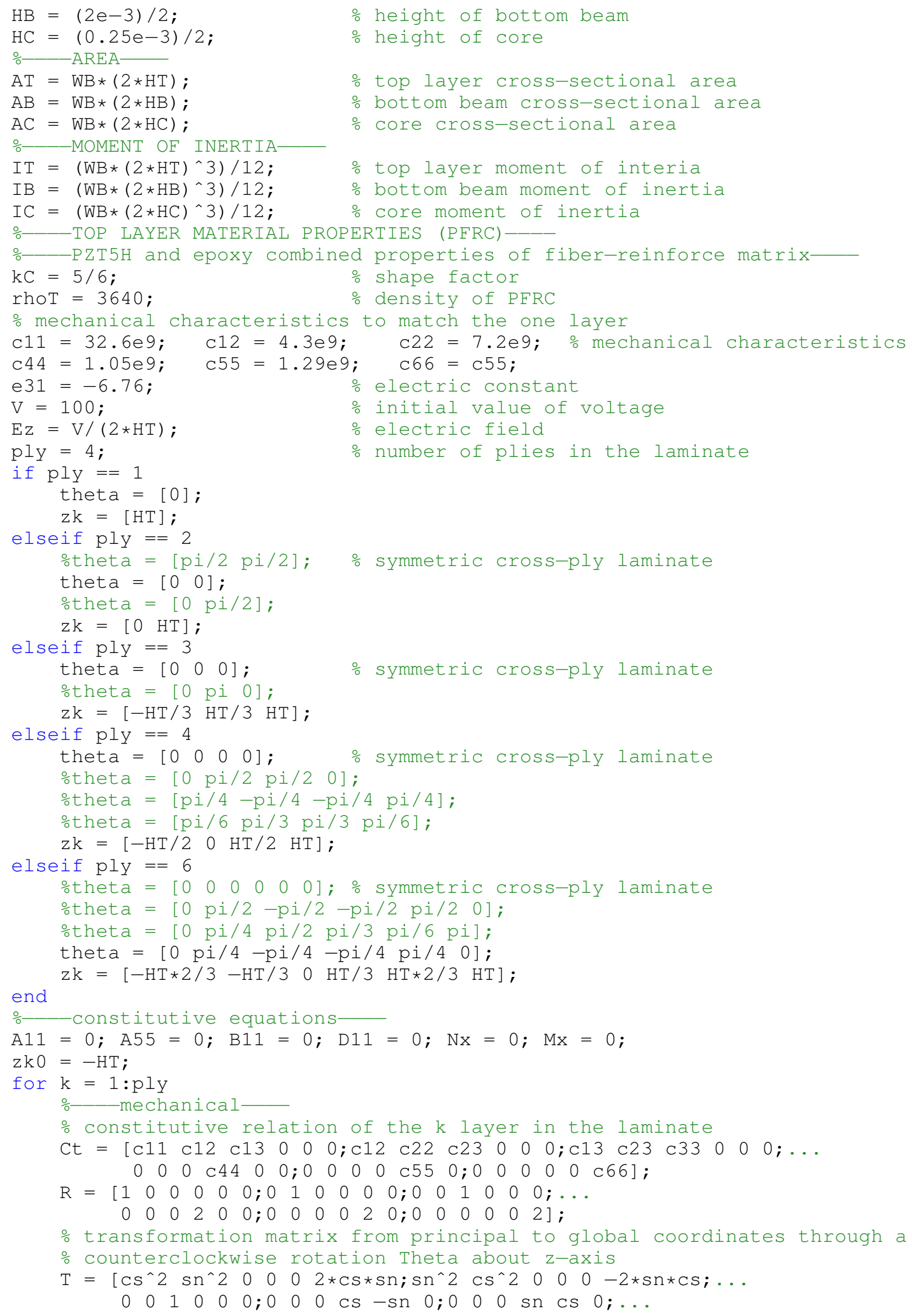




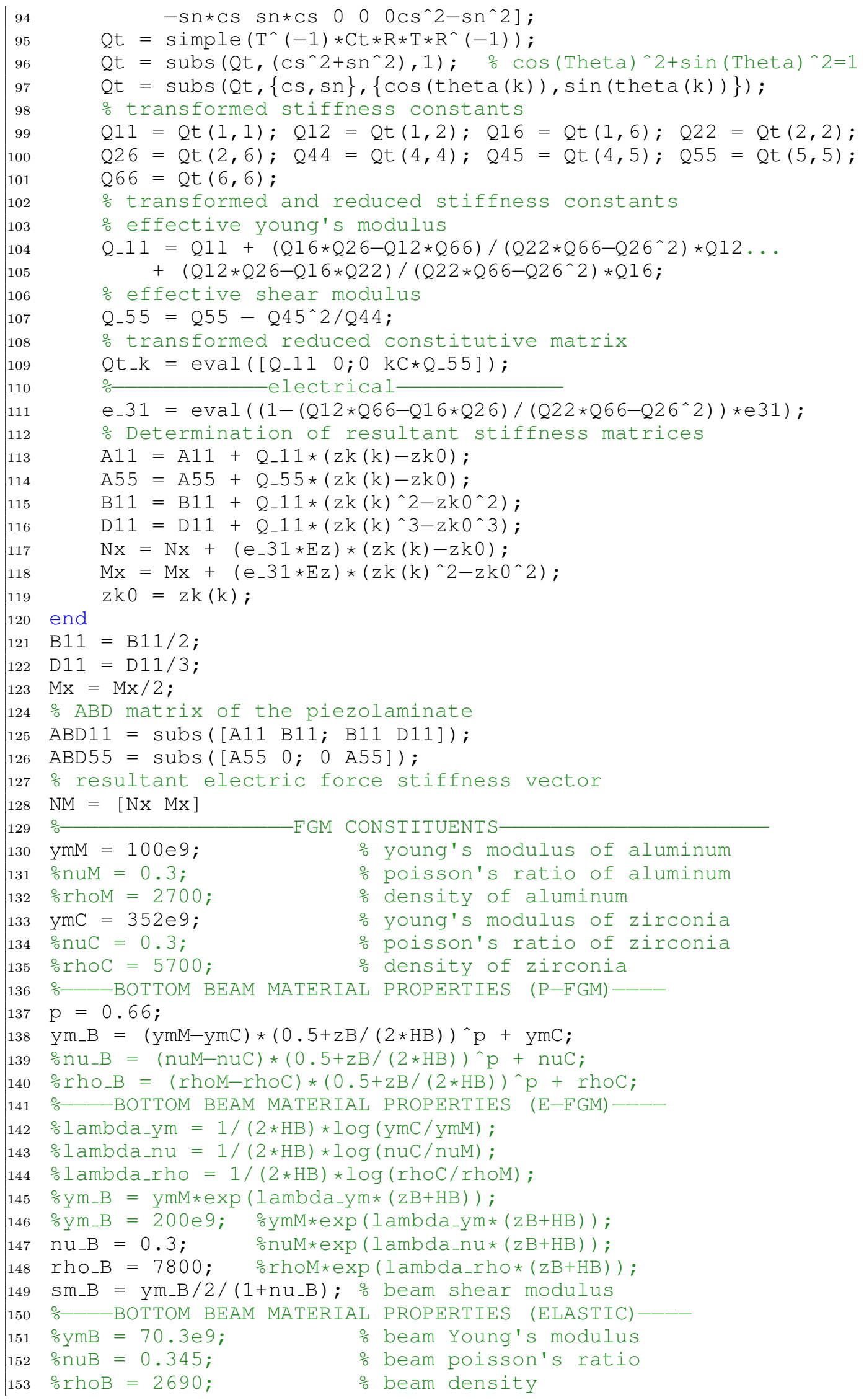




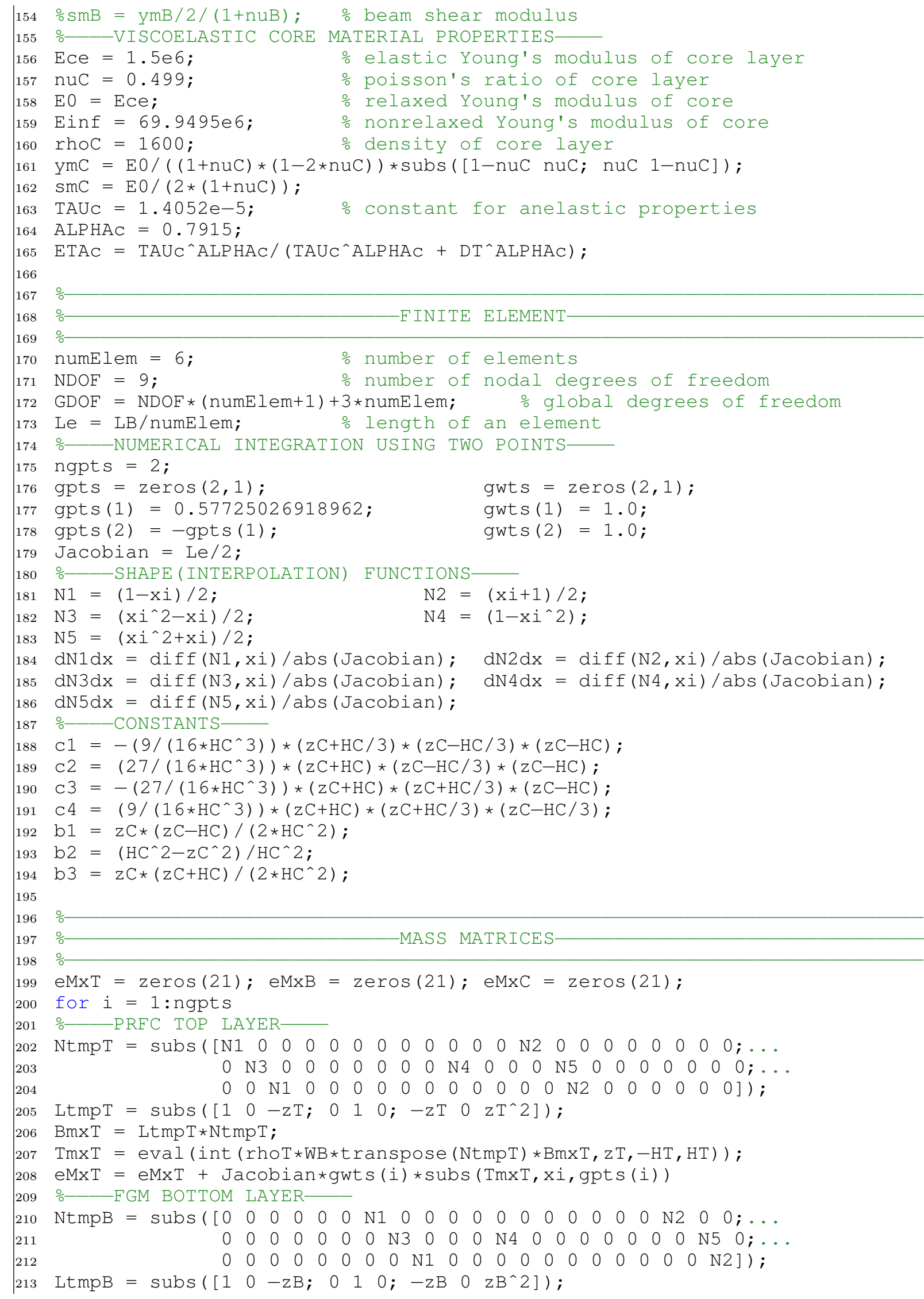




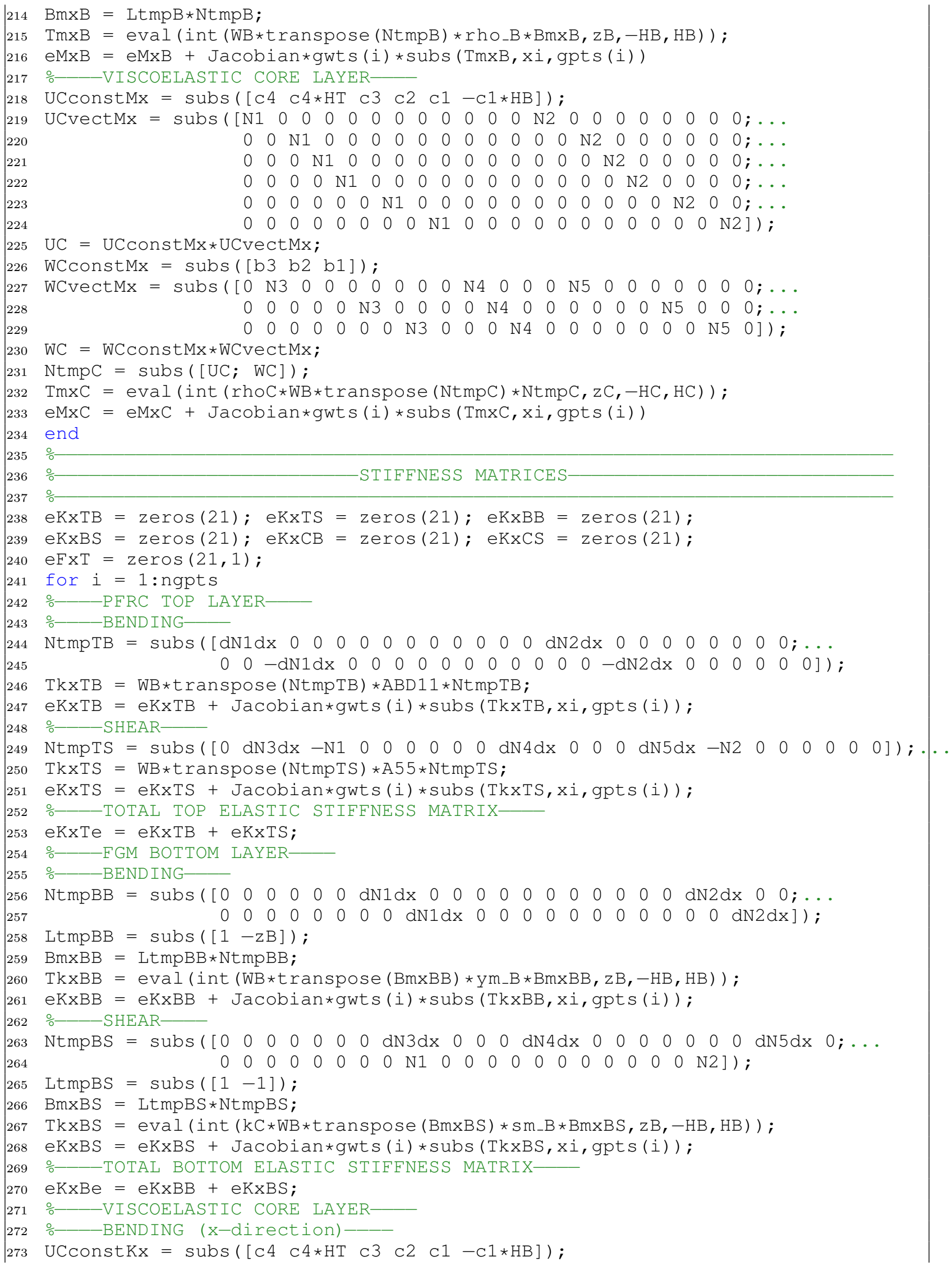




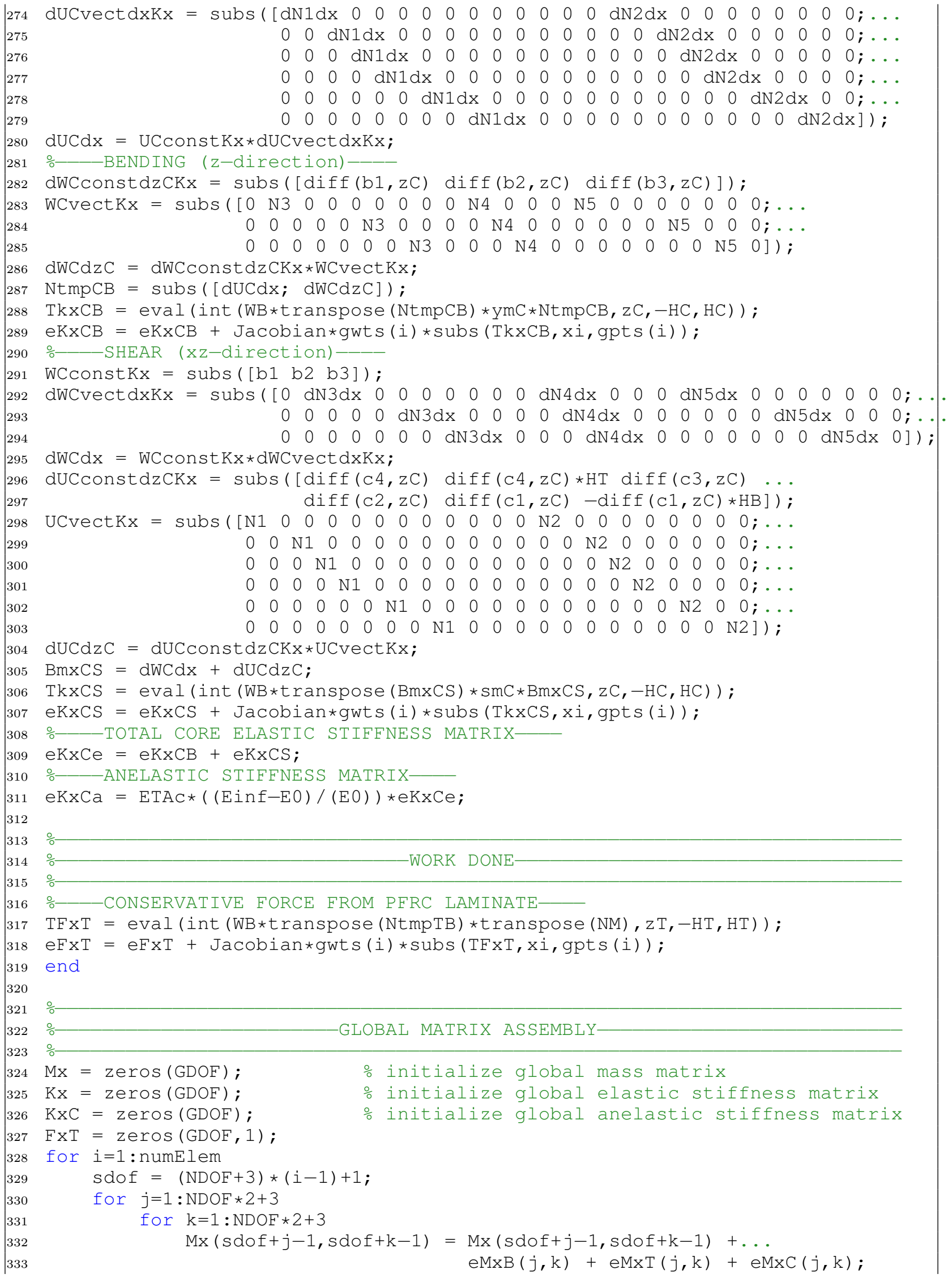




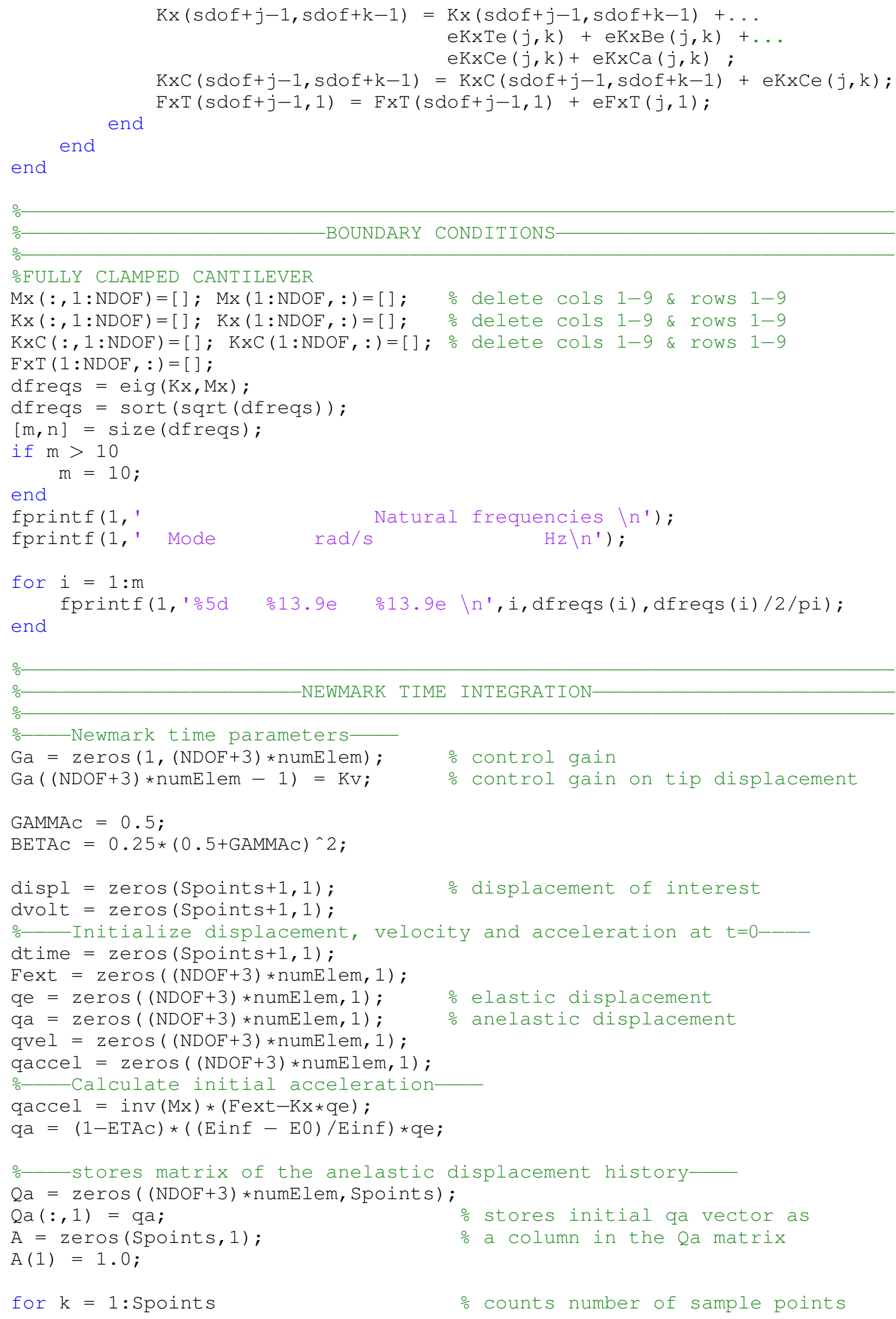




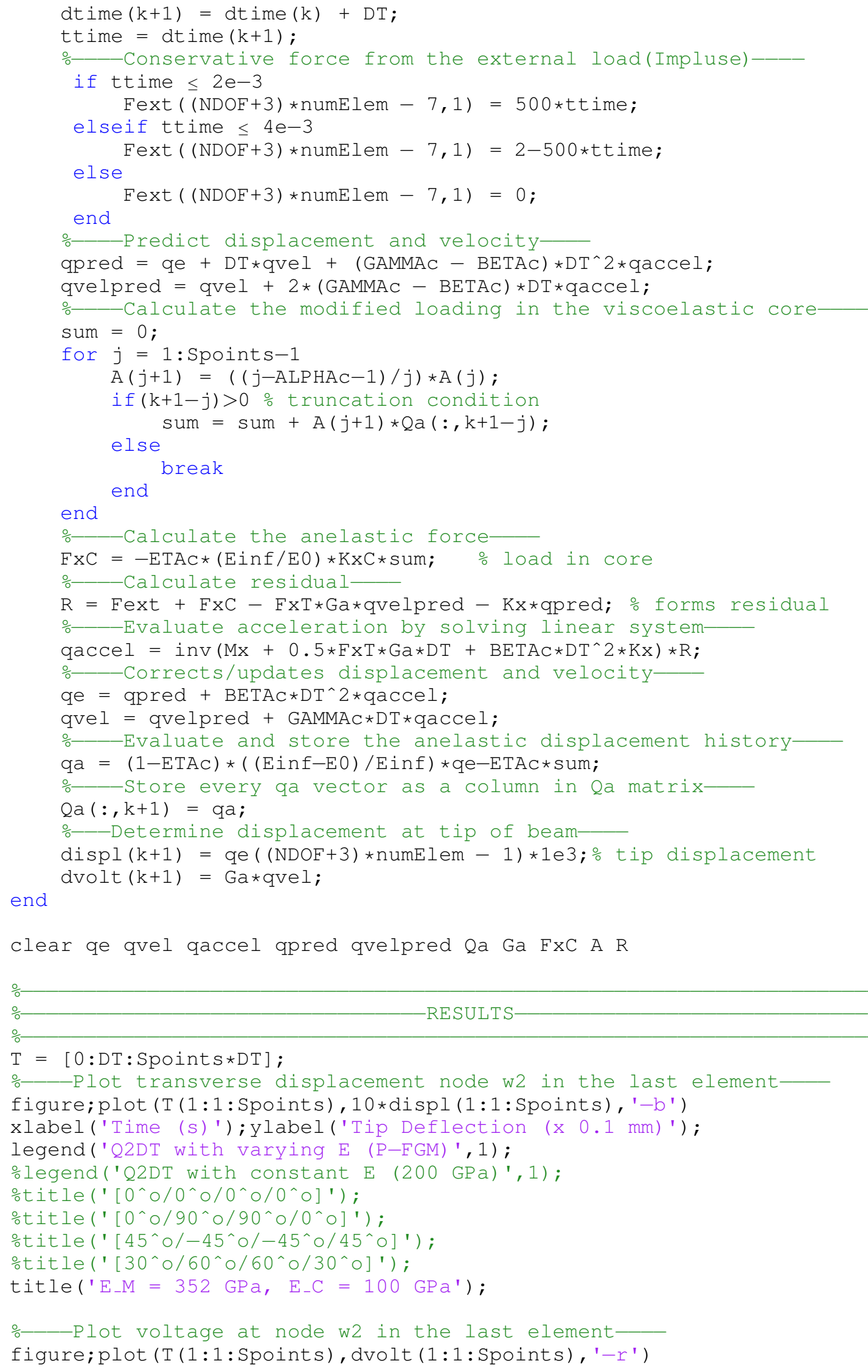


454 xlabel ('Time (S) ') ;ylabel ('Control Voltage (V) ') ;

455 \%legend('Q2DT fully-clamped cantilevered',1);

456 olegend('Q2DT with varying $\mathrm{E}(\mathrm{P}-\mathrm{FGM})$ ', 1);

457 \%legend('Q2DT with constant E (200 GPa)', 1);

458 otitle('[0^o/0^o/0^o/0^o]');

459 otitle ('[0^o/90^o/90^o/0^o]');

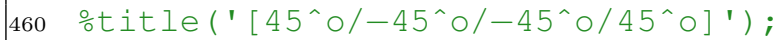

461 otitle ('[30^o/60^o/60^o/30^o]');

462 title('E_M $=436 \mathrm{GPa}, \mathrm{E}_{-} \mathrm{C}=70 \mathrm{GPa}$ ') ;

463 hold on

464

465 return 


\section{Bibliography}

[1] Stanway, R., Rongong, J.A., and Sims, N.D., "Active constrained-layer damping: a stateof-the-art review," Systems and Control Engineering, Vol. 217, 2003, Part I.

[2] Banerjee, J.R., and Sobey, A.J., "Dynamic stiffness formulation and free vibration analysis of a three-layered sandwich beam," International Journal of Solids and Structures, Vol. 42, 2005, pp. 2182-2197.

[3] Miroshnichenko, E., "A quasi-2D finite element formulation of active constrained-layer functionally graded beam," M.A.Sc. Thesis. Ryerson University, 2007

[4] Shi, Y., Hua, H., and Sol, H., "The finite element analysis and experimental study of beams with active constrained layer damping treatments," Journal of Sound and Vibration, Vol. 278, 2003, pp. 343-363.

[5] Bekuit, J-J.R.B., Oguamanam, D.C.D., and Damisa, O., "A quasi-2D finite element formulation for the analysis of sandwich beams," Finite Elements in Analysis and Design, Vol. 43, 2007, pp. 1099 - 1107.

[6] Bekuit, J-J.R.B., Oguamanam, D.C.D., and Damisa, O., "Quasi-2D finite element formulation of active-constrained layer beams," Smart Materials and Structures, Vol. 18, 2009, 095003(8pp).

[7] Rao, M.D., "Recent applications of viscoelastic damping for noise control in automobiles and commercial airplanes," Journal of Sound and Vibration, Vol. 262 No. 3, 2003, pp. 457-474.

[8] Balamurugan, V., and Narayanan, S., "Finite element formulation and active vibration control study on beams using smart constrained layer damping (SCLD) treatment," Journal of Sound and Vibration, Vol. 249 No. 2, 2002, pp. 227-250. 
[9] Gao, J.X., and Liao, W.H., "Vibration analysis of simply supported beams with enhanced self-sensing active constrained layer damping treatments," Journal of Sound and Vibration, Vol. 280, 2005, pp. 329-357.

[10] Trindade, M.A., and Benjeddou, A., "Hybrid active-passive damping treatments using viscoelastic and piezoelectric materials: review and assessment," Journal of Vibration and Control, Vol. 8, 2002, pp. 669-745.

[11] Trindade, M.A., Benjeddou, A., and Ohayon, R., "Piezoelectric active vibration control of damped sandwich beams," Journal of Sound and Vibration, Vol. 246 No. 4, 2001, pp. 653-677.

[12] Ray, M.C., and Sachade, H.M., "Exact Solution for the Functionally Graded Plates Integrated With a Layer of Piezoelectric Fiber-Reinforced Composite," Journal of Applied Mechanics, Vol. 73, 2006, pp. 622-632.

[13] Amirani, M.C., Khalili, S.M.R., and Nemati, N., "Free vibration analysis of sandwich beam with FG core using element free Galerkin Method," Composite Structures, Vol. 90, 2009, pp. 373-379.

[14] Rahmani, O., Khalili, S.M.R., Malekzadeh, K. and Hadavinia, H., "Free vibration analysis of sandwich structures with a flexible functionally graded syntactic core," Composite Structures, Vol. 91, 2009, pp. 229-235.

[15] Apetre, N.A., Sankar, B.V., and Ambur, D.R., "Impact Response of Sandwich Beam with Functionally Graded Core," AIAA Journal, Vol. 43, 2004, pp. 4358-4370.

[16] Apetre, N.A., Sankar, B.V., and Ambur, D.R., "Analytical Modeling of Sandwich Beams with Functionally Graded Core," Journal of Sandwich Structures and Materials, Vol. 10, 2008, pp. 53-74.

[17] Chi, S-H., and Chung, Y-L., "Mechanical behavior of functionally graded material plates under transverse load - Part I: Analysis," International Journal of Solids and Structures, Vol. 43, 2005, pp. 3657-3674.

[18] Galucio, A.C., Deii, J.-F., and Ohayon, R., "Finite element formulation of viscoelastic sandwich beams using fractional derivative operators." Journal of Computational Mechanics, Vol. 33, 2004, pp. 282-291.

[19] Welch, S.W.J., Rorrer, R.A.L., and Duren, Jr., R.G., "Application of time-based fractional calculus methods to viscoelastic creep and stress relaxation of materials," Journal of Mechanics of Time-Dependent Materials, Vol. 3, 1999, pp. 279-303. 
[20] Mallik, Nilanjan, and Ray, M.C., "Effective Coefficients of Piezoelectric FiberReinforced Composites," AIAA Journal, Vol. 41 No. 4, 2003, pp. 704-710.

[21] Sun, B., and Huang, D., "Vibration suppression of laminated composite beams with a piezo-electric damping layer," Composite Structures, Vol. 53, 2001, pp. 437-447.

[22] Zhang, X.D., and Sun, C.T., "Formulation of an adaptive sandwich beam," Smart Material Structures, Vol. 5, 1996, pp. 814-823.

[23] Agarwal, B.D., Broutman, L.J., and Chandrashekhara, K., "Analysis and Performance of Fiber Composites," John Wiley \& Sons, Third Edition 2006, pp.174-247.

[24] Hyer, M.W., "Stress Analysis of Fiber-Reinforced Composite Materials," McGraw-Hill, 1998, pp. 276-327.

[25] Kuo, B.C., "Automatic Control Systems: Eighth Edition," Prentice-Hall, 2002, pp. 438-442.

[26] Benjeddou, A., "Advances in Hybrid Active-Passive Vibration and Noise Control Via Piezoelectric and Viscoelastic Contrained Layer Treatments," Journal of Vibration and Control, Vol. 1, 2001, pp. 565-602.

[27] Bhimmaraddi, A., "Sandwich beam theory and the analysis of constrained layer damping," Journal of Sound and Vibration, Vol. 179 No. 4, 1995, pp. 591-602.

[28] Boresi, A.P., and Schmidt, R.J., "Advanced Mechanics of Materials," John Wiley E Sons, 6th Edition 2003, pp. 61-62.

[29] Chakraborty, A., Gopalakrishnan, S., and Reddy, J.N., "A new beam finite element for the analysis of functionally graded materials," International Journal of Mechanical Sciences, Vol. 45, 2003, pp. 519-539.

[30] Chandrashekhara, K., and Agarwal, A.N., "Active Vibration Control of Laminated Composite Plates Using Piezoelectric Devices: A Finite Element Approach," Journal of Intelligent Material Systems and Structures, Vol. 4, 1993, pp. 496-508.

[31] Cook, R.D., Malkus, D.S., Plesha, M.E., and Witt, R.J., "Concepts and Applications of Finite Element Analysis," John Wiley 6 Sons, Fourth Edition 2002, pp. 416-426.

[32] Gopinathan, S.V., Varadan, Vasundara, and Varadan, Jijay, "A review and critique of theories for piezoelectric laminates," Smart Material Structures, Vol. 9, 2000, pp. 24-48. 
[33] Kiral, Z., Malgaca, L., and Akdag, M., "Active Control of Residual Vibrations of a Cantilever Smart Beam," Turkish Journal of Eng. Env. Sci., Vol. 32, 2008, pp. 51-57.

[34] Kirugulige, M.S., Kitey, R., and Tippur, H.V., "Dynamic fracture behavior of model sandwich structures with functionally graded core: a feasibility study," Composite Science and Technology, Vol. 65, 2005, pp. 1052-1068.

[35] Li, F.-M., Kishimoto, K., Wang, Y.-S., Chen, Z.-B., and Huang, W.-H., "Vibration control of beams with active constrained layer damping," Smart Material Structures, Vol. 17, 2008, pp. 1-9.

[36] Li, X.-F., "A unified approach for analyzing static and dynamic behaviors of functionally graded Timoshenko and Euler-Bernoulli beams," Journal of Sound and Vibration, Vol. 318, 2008, pp. 1210-1229.

[37] Li, Q., Iu, V.P., and Kou, K.P., "Three-dimensional vibration analysis of functionally graded material sandwich plates," Journal of Sound and Vibration, Vol. 311, 2008, pp. 498-515.

[38] Liew, K.M., Sivashanker, S., He, X.Q., and Ng, T.Y., "The modelling and design of smart structures using functionally graded materials and piezoelectrical sensor/actuator patches," Smart Materials and Structures, Vol. 12, 2003, pp. 647-655.

[39] Logan, D.L., "A First Course in the Finite Element Method," Thomson Canada Ltd., Fourth Edition 2007, pp. 305-322, 463-470 \& 659-661.

[40] Sankar, B.V., "An elasticity solution for functionally graded beams," Composites Science and Technology, Vol. 61, 2001, pp. 689-696.

[41] Xiang, H.J., and Yang, J., "Free and forced vibration of laminated FGM Timoshenko beam of variable thickness under heat conduction," Composites Part B:Engineering, Vol. 39, 2008, pp. 292-303.

[42] Yang, L., and Zhifei, S., "Free vibration of a functionally graded piezoelectric beam via state-space based differential quadrature," Composite Structures, Vol. 87, 2009, pp. 257-264. 